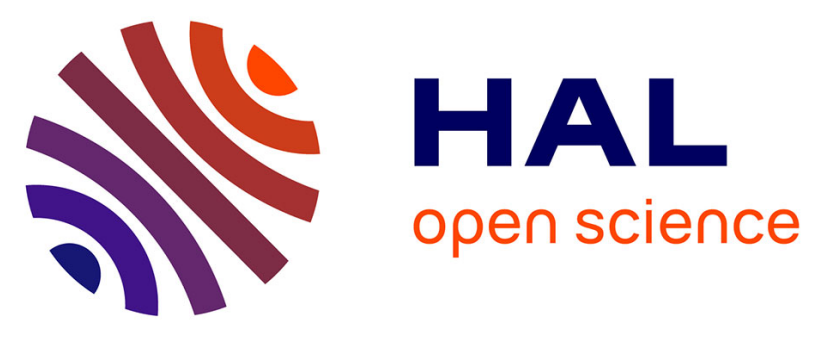

\title{
Pre-orogenic upper crustal softening by lower greenschist facies metamorphic reactions in granites of the central Pyrenees
}

Laura Airaghi, Nicolas Bellahsen, Benoît Dubacq, David M Chew, Claudio L Rosenberg, Emilie Janots, Maxime Waldner, Valérie Magnin

\section{To cite this version:}

Laura Airaghi, Nicolas Bellahsen, Benoit Dubacq, David M Chew, Claudio L Rosenberg, et al.. Preorogenic upper crustal softening by lower greenschist facies metamorphic reactions in granites of the central Pyrenees. Journal of Metamorphic Geology, 2020, 38 (2), pp.183-204. 10.1111/jmg.12520 . insu-02477010

\section{HAL Id: insu-02477010 \\ https://hal-insu.archives-ouvertes.fr/insu-02477010}

Submitted on 13 Feb 2020

HAL is a multi-disciplinary open access archive for the deposit and dissemination of scientific research documents, whether they are published or not. The documents may come from teaching and research institutions in France or abroad, or from public or private research centers.
L'archive ouverte pluridisciplinaire HAL, est destinée au dépôt et à la diffusion de documents scientifiques de niveau recherche, publiés ou non, émanant des établissements d'enseignement et de recherche français ou étrangers, des laboratoires publics ou privés. 


\section{Pre-orogenic upper crustal softening by lower greenschist- facies metamorphic reactions in granites of the central Pyrenees}

\begin{tabular}{|r|l|}
\hline Journal: & Journal of Metamorphic Geology \\
\hline Manuscript ID & JMG-19-0021.R1 \\
\hline Manuscript Type: & Original Article \\
\hline Author: & 27-Sep-2019 \\
\hline Complete List of Authors: & $\begin{array}{l}\text { Airaghi, Laura; Institut des sciences de la terre de Paris, Sorbonne } \\
\text { Université } \\
\text { Bellahsen, Nicolas; Institut des sciences de la terre de Paris, Sorbonne } \\
\text { Université } \\
\text { Dubacq, Benoît; Institut des sciences de la terre de Paris, Sorbonne } \\
\text { Université } \\
\text { Chew, David; Trinity College Dublin, Geology } \\
\text { Rosenberg, Claudio; Institut des sciences de la terre de Paris, Sorbonne } \\
\text { Université } \\
\text { Janots, Emilie; Observatoire des Sciences de I'Univers de Grenoble, } \\
\text { Institut des Sciences de la Terre } \\
\text { Waldner, Maxime; Institut des sciences de la terre de Paris, Sorbonne } \\
\text { Université } \\
\text { Magnin, Valérie; Observatoire des Sciences de l'Univers de Grenoble, } \\
\text { Institut des Sciences de la Terre }\end{array}$ \\
\hline Keywords: & $\begin{array}{l}\text { U-Th/Pb anatase-titanite-monazite dating, mylonites, chlorite-white mica } \\
\text { thermobarometry, Bielsa, Axial zone }\end{array}$ \\
\hline
\end{tabular}


1 Pre-orogenic upper crustal softening by lower greenschist-facies metamorphic reactions

2 in granites of the central Pyrenees

3

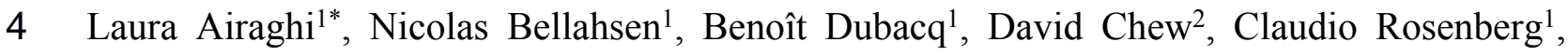
5 Emilie Janots ${ }^{3}$, Maxime Waldner ${ }^{1}$, Valérie Magnin ${ }^{3}$

6

$7 \quad{ }^{1}$ Sorbonne Université, CNRS-INSU, Institut des Sciences de la Terre de Paris, ISTeP UMR

8 7193, F-75005 Paris, France

$9 \quad{ }^{2}$ Department of Geology, Trinity College Dublin, Dublin 2, Ireland

$10{ }^{3}$ Université Grenoble Alpes, CNRS, IRD, IFSTTAR, ISTerre, 38000 Grenoble, France

11

12 *corresponding author:laura.airaghi@univ-orleans.fr, now at: Université d'Orléans, CNRS-

13 INSU, Institut des Sciences de la Terre d'Orléans, ISTO, UMR7327, Orléans, France

14

15

16 Short title: Pre-kinematic metamorphism in Pyrenees

17

18

19

20

21

22

23

24 


\section{ABSTRACT}

Pre-kinematic greenschist-facies metamorphism is often observed in granites and basement units of mountains belts, but rarely dated and accounted for in orogenic cycle reconstructions. Studying pre-kinematic alteration is challenging because of its usual obliteration by subsequent syn-kinematic metamorphism often occurring at conditions typical of the brittle-ductile transition. It is however to be expected that pre-kinematic alteration has major implications for the rheology of the upper crust. In the 305 Ma-old Variscan basement of the Bielsa massif (located in the Axial Zone of the Pyrenees), successive fluid-rock interaction events are recorded in granites at temperatures below $350^{\circ} \mathrm{C}$. Combined microstructural and petrographic analysis, low-temperature thermobarometry and in situ $\mathrm{U}-\mathrm{Th} / \mathrm{Pb}$ dating of anatase, titanite and monazite show extensive pre-orogenic (pre-Alpine) and pre-kinematic alteration related to feldspar sericitization and chloritization of biotite and amphibole at temperatures of $270-350^{\circ} \mathrm{C}$ at 230-300 Ma. This event is followed by a second fluid-rock interaction stage marked by new crystallisation of phyllosilicates at temperatures of $200-280^{\circ} \mathrm{C}$ and associated with the formation of mylonitic shear zones and fractures parallel to the shear planes. U-Pb anatase and monazite ages as well as the microtextural relationships of accessory minerals suggest an age for this event at 40-70 Ma, consistent with independent regional geology constraints. The

44 Variscan basement was therefore softened at late- to post Variscan time, at least 150-200 Ma 45 before the main Alpine shortening while Alpine-age compression lead to the formation of a dense net of mylonites. The associated deformation, both distributed at the scale of the Bielsa massif and localized at decametric scale in mylonitic corridors, precedes the strain localization along the major thrusts of the Axial zone. The Bielsa massif is a good example where, inherited, 

characterised, and hardly ever dated and placed in a regional orogenic context. Indeed, low-

pre-orogenic upper crustal softening controls the deformation patterns in granitic basement units through low-grade metamorphic reactions.

Key words: $\mathrm{U}-\mathrm{Th} / \mathrm{Pb}$ anatase-titanite-monazite dating, mylonites, chlorite-white mica thermobarometry, Bielsa, Axial zone

\section{INTRODUCTION}

Granites deformed at the brittle-ductile transition show mineral assemblages characteristic of low-grade metamorphism. Greenschist-facies metamorphic reactions often result in the growth of phyllosilicates (e.g. white mica, chlorite) whose presence strongly affect the rheological behaviour of the upper crust through softening, strain localization and fluid migration (Tullis, Yund, \& Farver, 1996; Ingles, Lamouroux, Soula, Guerrero, \& Debat, 1999; Jackson, Austrheim, McKenzie, \& Priestley, 2004; Wintsch, Christoffersen, \& Kronenberg, 2005; Goncalves, Oliot, Marquer, \& Connolly, 2012). The breakdown of $80 \%$ of feldspar into white mica, for example, reduces the depth of the brittle-ductile transition by two thirds (Gueydan, Leroy, Jolivet, \& Agard, 2003). Reactions forming low-grade mineral assemblages responsible for crustal softening are generally observed to be syn-kinematic, occurring during the major deformation phases of an orogenic cycle (Wayne \& McCaig, et al., 1998; Gueydan et al., 2003; Mansard, Raimbourg, Augier, Précigout, \& Le Breton, 2018). Static or quasi-static hydrothermal alteration is also observed in granites and has been widely documented, especially in large igneous provinces (e.g. Parneix, Beaufort, Dudoignon, \& Meunier, 1985; Cathelineau et al., 1986; Yuguchi, Sasao, Ishibashi, \& Nishiyama, 2015). However static alteration is rarely grade metamorphic reactions often leave tenuous fingerprints only hinted at by equivocal 
74 microtextural relationships, especially in granites (e.g. Airaghi et al., 2017b), which are

75 generally considered poorly reactive rocks at temperatures below $400^{\circ} \mathrm{C}$.

At a microscopic scale, pre-kinematic and syn-kinematic transformations are difficult

to distinguish, particularly so if the rocks have been deformed through several orogenic cycles.

78 The timing and conditions of pre-kinematic metamorphic reactions and their effects on (i) the syn-kinematic transformation and (ii) the rheological response of the upper crust to successive compressive phases remain therefore poorly constrained. This study addresses these issues by

81 focussing on the Bielsa massif, a Variscan granite located in the Axial Zone of the central Pyrenees (Figure 1a). This massif is an excellent natural laboratory to study how granitic rocks record distinct low-grade metamorphic events. The massif is part of the central Pyrenean belt, 84 which experienced at least two well-known deformation phases (Late Variscan and 85 Alpine/Pyrenean, Cochelin et al. 2017), which are difficult to distinguish in the Axial zone 86 (Cochelin et al, 2017). The Bielsa massif is located in the most external basement domain of 87 the Pyrenees (implying it experienced the lowest peak metamorphic temperatures with respect 88 to Alpine tectonics), south of the zones of large-scale shear bands that have affected other 89 granitic basement units of Pyrenees (e.g. Maladeta, Néouvielle; Wayne \& McCaig, 1988). In addition, structural and microstructural studies show significant distributed shortening at the

91 scale of the massif (Román-Berdiel et al., 2004; Bellahsen et al., 2019), raising questions about 92 the link between low-grade metamorphism and strain accommodation mechanisms. Early 93 feldspar destabilisation has been proposed to have a major control on subsequent mechanical 94 softening in Bielsa (Bellahsen et al., 2019), but the conditions and the age of such reaction still remains unconstrained. In this contribution we estimate the conditions of crystallization of both pre-kinematic and syn97 kinematic greenschist-facies mineral assemblages in the Bielsa granite and its Triassic 98 sedimentary cover. Microstructural and petrographic analyses were combined with low- 
99

100

101

102

103

104

105

106

107

108

109

110

111

112

113

114

115

116

temperature thermobarometry and in situ $\mathrm{U}-\mathrm{Th} / \mathrm{Pb}$ dating of zircon, titanite and monazite. Attempt to date anatase (polymorph of rutile) by the U/Pb method were also undertaken. The implications for the timing of crustal softening relative to the major shortening phases are discussed with respect to their associated mineral assemblages. Results suggest that prekinematic (pre-Alpine) alteration controls the deformation pattern and strain localisation in the Bielsa granite during Alpine shortening.

\section{THE BIELSA MASSIF IN THE AXIAL ZONE (CENTRAL PYRENEES)}

The Axial zone (central Pyrenees) is part of a S-verging thrust system made of imbricated thrust units stacked during the Late Cretaceous-Early Miocene collision of the Iberian and European plates (Figure 1a). The plutons of the Axial zone (i.e. Bielsa, Neuville, Maladetta massifs etc., Figure 1a) were emplaced into Paleozoic sediments during LateVariscan (320-300 Ma, e.g. Paquette, Gleizes, Leblanc, \& Bouchez, 1997; Vacherat et al., 2017).

These plutons and their Paleozoic sedimentary cover constitute the basement to the Permian and Mesozoic sedimentary units (Fig. 1a). Several tectono-metamorphic stages are documented in the metasedimentary units Axial zone but are difficult to distinguish within the basement. Recent studies have proposed that the majority of the deformation in the Axial zone is of Variscan age (Cochelin et al., 2017). Cretaceous hydrothermal events have been identified in the eastern Axial zone (Fallourd et al., 2014; Boutin et al., 2015) and related to extensive tectonics centered on the SE Bay of Biscay. Basement thrusts in the central-western Pyrenees (named Gavarnie and Guarga thrusts) were (re)activated during the Eocene to Miocene (20-30 and 32-36 Ma respectively, Jolivet et al., 2007; Elmola et al., 2018 and references therein). In the hanging wall of the Gavarnie thrust, an older deformation event (c. $48 \mathrm{Ma}$ ) is recognised in the massifs of Néouvielle and Eaux Chaudes. It has been associated with the activation of mylonitic shear bands (e.g. Wayne \& McCaig, 1998). 
124 This study focusses on the Bielsa massif, mainly constituted by a pluton emplaced at c. $305 \mathrm{Ma}$

125 (Vacherat et al., 2017), in the S-verging thrust system structurally located between the Gavarnie 126 and Guarga thrusts (Figure 1b). Close to Lake Urdiceto (Figure 1c-d), granitic to granodioritic 127 rocks (Román-Berdiel et al., 2004) are unconformably overlain by Triassic continental red 128 sandstones and pelites. The basement and its sedimentary cover were deformed (folded) during 129 the Alpine compressional phase (Figure 1d, Casas et al., 2003; Román-Berdiel et al., 2004, 130 Bellashen et al., 2019) with a wavelength of $\sim 1 \mathrm{~km}$. Several sub-vertical S- or N-dipping normal 131 or reverse steep faults bound the antiforms and synforms (Figure 1d). In the core of the synforms, 132 Triassic sediments crop out. They exhibit a sub-vertical to N-dipping cleavage (e.g. Figure 2b 133 and 3c), with nord side up sense of shear kinematic indicators above the sediment-cover 134 interface (Bellahsen et al., 2019). The granitic basement is heterogeneously deformed. At the 135 decametric scale, undeformed and weakly deformed granitic rocks alternate with zones of 136 distributed ductile deformation, zones of fracturing and faulting and mylonites (Figures 1c-d and 2a-f). A pervasive N-dipping schistosity is widespread in the basement (Figure 2a-c and e).

138 Mylonitic corridors, with 1-2 m wide, are observed within the basement and at its boundary 139 with the Triassic sedimentary cover (Figure 2a-c). Mylonitic corridors alternate with less 140 deformed rocks with a wave length of $\sim 100 \mathrm{~m}$ (Figure 1c) and they are characterized by 141 pervasive schistosity (S) and steep, N-dipping $\left(45-70^{\circ}\right)$ and S-dipping $\left(55-70^{\circ}\right)$ shear planes (C) associated either with a north or with a south-verging movement (Figure 2a-e). In some shear 143 zone, the dips of mylonitic planes varies from S-dipping to N-dipping, with an apparent sense 144 of shear from top-to-north to top-to-south (Figure 2b-d). Some of the shear bands at the 145 boundary between the Triassic sediments and the late Variscan granite (regionally trending 146 N110, Figure 1c) contain deformed fragments of the Triassic red sandstone (Román-Berdiel et 147 al., 2004; Figure 2b), indicating that they are post-Triassic. The high density of mylonitic corridors and the pervasive schistosity development result in a distributed deformation pattern 
149 (as observed in other crystalline units as the Aar massif, Wehrens et al., 2017). The 150 granitic/granodioritic basement (regardless of the intensity of the strain) is pervasively hydrated 151 and the original magmatic mineral assemblage is partly to totally replaced by greenschist-facies 152 minerals. The steep topography of the massif allows one to infer that the latter replacement 153 affects the pluton at least over a topographic vertical profile of $2 \mathrm{~km}$ from the lowest outcrop 154 of the massif (yellow stars in Figures 1b).

At the boundary between the Triassic sediments and the granite/granodiorite, subhorizontal quartz and chlorite-bearing veins which are 1-5 cm-wide are observed (yellow square in Fig. 3b). Fifty meters from the contact, Triassic sediments show two generations of chloriteand quartz-bearing veins: (1) sub-horizontal veins parallel to the ones observed at the contact between the basement and the sedimentary rocks produced by vertical extension, locally folded and associated with a crenulation cleavage (N184S) and (2) sub-vertical veins (Figure 3c).

\section{SAMPLE DESCRIPTION AND CHRONOLOGY OF MINERAL ASSEMBLAGES}

Twenty-seven samples were collected along two transects east and west of Lake Urdiceto, from the undeformed granite (e.g. ZA16-84 and B11-16, Figure 4a, b) up to the zone of intense mylonitisation (e.g. ZA16-96, ZA16-98; Figures 1c, 4g). One sample (ZAL18-11) was collected at the contact between the Triassic sandstones and a basement-hosted vein (Figures 3b). Two samples (ZA16-12 and ZA16-13) were collected in the Triassic sediments (Figures $3 c)$.

\subsection{Samples from the basement}

171 In the basement unit, we distinguished the following rock fabrics: Undeformed samples (e.g. 172 ZA16-84, Figure 4a), where the magmatic, euhedral shape of grains is still preserved. Weakly 173 deformed rocks (e.g. Figure $2 \mathrm{f}$ and $4 \mathrm{c}$ ) exhibiting a weak yet pervasive schistosity (at an outcrop 174 scale: $\mathrm{S}$ in Figure 2a). In some of those samples the schistosity is associated to mm-scale 
175 fractures (e.g. ZA16-82, ZA16-78, ZA16-128, Figure 4e-f and h) that are parallel to the 176 subvertical shear planes observed at an outcrop scale in mylonites ( $\mathrm{C}$ in Figure 2a). Mylonites 177 showing S and C planes (e.g. ZA16-133, Figure 4g), where the C planes are as pervasive as S. 178 In the samples defined as mylonite, both brittle and ductile deformation features are identified, 179 with the brittle ones underlined by fractures parallel to the shear planes C (Figure 4g).

All samples, regardless of the degree of the strain, exhibit extensive alteration of the magmatic mineral assemblage originally defined by quartz, K-feldspar and plagioclase, biotite \pm amphibole, apatite, zircon (all in textural equilibrium, Figures 4a-b) and ilmenite preserved as inclusions in the less altered amphibole and biotite grains (Figure 5a-b). The alteration is comprised by the breakdown of plagioclase and K-feldspar into white mica (10-30 $\mu \mathrm{m}$ in size) + albite + calcite \pm rare epidote (Figure $4 \mathrm{a}, \mathrm{c}, \mathrm{e}-\mathrm{f}$ ). Biotite and amphibole break down to chlorite flakes (ChlA, abbreviations are from Whitney \& Evans, 2010) \pm white mica $(20-100 \mu \mathrm{m}$ in size) \pm prehnite (Figure 4a) and a Ti-bearing phase (anatase or titanite or both, Figure 4b, d, f and g). In the paragraph below, mineral assemblages are described in relation to their microstructural evolution from the undeformed granites and granodiorites to mylonites.

190 In the undeformed samples, relicts of feldspar, biotite and amphibole are still visible and their 191 euhedral magmatic grain shapes are preserved (Figure 4a). Chlorite flakes (ChlA) grow at the grain boundaries of parent minerals and along biotite cleavages, and they are associated with a Ti-bearing phase (see below) \pm prehnite \pm K-feldspar (Figure 4a-b). Ilmenite grains are 194 preserved in unaltered biotite and amphibole domains (Figure 5a). Quartz grains contain sparse, 195 micrometric fluid inclusions and are cross-cut by microcracks. Allanite is locally observed in 196 textural equilibrium with the magmatic minerals. Euhedral magmatic zircon grains are found 197 within relicts of feldspar or at the boundaries between feldspar and amphibole. 198 Cathodoluminescence imaging of zircon in samples ZA16-84 and ZA16-119 typically reveals 199 primary oscillatory growth zoning without internal complexity. However, some zircon grains, 
200 especially in sample ZA16-119, show metamictization with porous domains and lobate rims 5-

$20120 \mu \mathrm{m}$ thick, and very low cathodoluminescence emission (Figure 5d). In the weakly deformed 202 samples, feldspar is pervasively sericitized (up to 80-90\%). Original biotite grains are 203 chloritized between 70-90\% (Figure 4c). Chlorite flakes (ChlA) and secondary reaction phases 204 (quartz, Ti-phase \pm white mica) are oriented parallel to the main schistosity and tightly folded 205 to form kinks (Figure 4d). Quartz grains partly preserve their magmatic texture, but are 206 fractured, with fractures subparallel to the kink axes. Grains are cross-cut by fluid inclusion 207 trails. Originally euhedral grains of ilmenite are first altered to aggregates of anatase + calcite 208 then to titanite (Figure 5a-b), following the reaction proposed by Hansen, Reimink, \& Harlov 209 (2010) for low temperature conditions. Anatase is itself replaced by titanite, in textural 210 equilibrium with chlorite flakes (Figures 5a-b). Anatase and titanite are systematically aligned 211 with the chlorite flakes, within the S-planes. When they are folded to form kinks, anatase relicts are mainly preserved in the microfold hinges (Figure 5c). In some of the weakly deformed samples, titanite is the major Ti-bearing phase preserved (samples ZA16-119, B11-16, ZA1677 and ZA16-84).

In weakly deformed samples showing millimetric fractures, biotite deformation and

216 stretching along the main schistosity $\mathrm{S}$ is more pronounced (e.g. Figure 4e). Calcite is present 217 in the matrix. Fractures 1-3 mm thick displace the folded chlorite flakes to form en-echelon 218 microstructures (Figure 4e). These fractures are filled with secondary chlorite $(\mathrm{ChlB}) \pm$ white 219 mica (Figure 4f). Sheets of ChlB are oriented parallel to the fracture walls in contrast to ChlA 220 grains which are mainly perpendicular (Figure 4f). Furthermore, ChlB is rarely associated with 221 biotite breakdown products as prehnite and Ti-bearing phases (Figure 4f). Quartz grains are both fractured and stretched, they exhibit undulose extinction and abundant fluid inclusion trails.

223 Titanite is observed in the same microstructural position as in undeformed samples (folded 224 within ChlA flakes). The microstructural position of anatase is instead more variable. Anatase 
is observed: (1) as $\sim 70 \mu \mathrm{m}$ sub-euhedral relicts in titanite cores at the rims of the ChlA flakes

226 (as in the undeformed samples, type-1 Ant, Figure 4h, 5b-c, f-h and Figure S1) and (2) as grains

20-30 $\mu \mathrm{m}$ in size not associated with titanite within ChlA flakes along the ancient biotite cleavages (type-2 Ant, e.g. Figure 4h, 5f-h and Figure S1). Locally, type-2 anatase is observed within ChlB-bearing fractures (e.g. ZA16-73, Figure 5i). Type-2 anatase of type 2 is often observed close to type-1 anatase. Magmatic accessory minerals such as apatite and zircon are 231 fragmented and stretched along the $\mathrm{S}$ planes.

In mylonitic samples, the schistosity is defined by folded chlorite + white mica flakes and interconnected layers of white mica (Figure 4g). Flakes are folded up to kinks. Shear planes

234 (C) form at high angle with the main schistosity $\mathrm{S}$ (Figure 4g-h). The kink axes are 235 kinematically compatible with shear movement on the C planes. Quartz grains are partially 236 stretched and most of them are fractured, with cracks subparallel to the C planes (Figure 4g). 237 Within the grains, evidences of ductile deformation are observed such as deformation lamellae.

238 A larger amount of fluid inclusions in quartz are observed with these samples compared to 239 weakly deformed samples. In mylonites (ZA16-96, ZA16-98), only one type of anatase is 240 observed (small anatase grains of type 2 predominate). In these samples, monazite grains, 10$24140 \mu \mathrm{m}$ in size, were identified in mylonites (sample ZA16-98 and ZA16-88). These monazite grains are wrapped by secondary chlorite and white mica (suggesting post-magmatic origin).

243 The largest grains of monazite $(20-40 \mu \mathrm{m})(\mathrm{Mnz} 1)$ are lobate, and stretched along the C planes

244 with fractures perpendicular to them and exhibit irregular grain boundaries, micrometric large 245 fractures, patchy textures with bright and dark spots in BSE images and inclusion-like 246 dissolution figures' (as described by Grand'Homme et al., 2018; Airaghi, Janots, Lanari, de 247 Sigoyer, \& Magnin, 2018, Figure 5c). Small monazite grains $(\sim 10 \mu \mathrm{m})$ with rounded shape 248 share features with that of ZA16-88, aligned along the main foliation or within alteration halos 249 marking the reaction front along secondary fractures subparallel to the C (Figure 5j). In sample 
250 ZA16-98 monazite is locally Th and Si-enriched (tending towards a thorite composition, inset

251 in Figure 5i).

In summary, the granitic rocks experienced a first transformation of the original

253 magmatic mineral assemblage of $\mathrm{Qz}+\mathrm{Fs}+\mathrm{Pl}+\mathrm{Bt} \pm \mathrm{Amph}(+\mathrm{Ilm}+\mathrm{Ap}+\mathrm{Zr} \pm \mathrm{Aln})$ to Qz +

254 ChlA + Ti-phase (Ttn or Ant then Ttn) $\pm \operatorname{Pr} \pm$ KFs. Zircon and titanite grains with anatase core

255 (type-1 Ant) are stretched in the main schistosity and folded during shearing. They therefore

256 predate any deformation stage. ChlB is rarely associated with secondary reaction products and

257 helps to define the C-parallel fractures. It is considered to precipitate from a fluid during a

258 second syn-kinematic (syn C planes) alteration event. The varying characteristics of anatase,

259 only found in deformed samples, suggest that in these samples two generations of anatase may

260 be preserved. That type-2 anatase is located within ChlA flakes along former biotite cleavage

261 suggests that type-2 Ant grew after type-1 Ant on ChlA rims. The chloritization reaction front

262 and replacement is indeed expected to progress from the grain boundaries to the core, along the

263 biotite cleavages. The proximity of type-2 anatase to type- 1 anatase grains indicates however

264 transport scale of the anatase-forming elements limited to some dozen of $\mu \mathrm{m}$. In sample ZA16-

26573 (discretely deformed). Type-2 Ant is observed in C-parallel fractures (Figure 5i). Type-2

266 Ant may have crystallized during the shearing. Mnzl is affected by deformation and mainly

267 observed within the main schistosity, while Mnz2 (and type-2 Ant, see below) are associated

268 with C parallel fractures. Mnz2 is therefore syn C planes forming stage.

2703.2 Samples from Triassic sediments and at the boundary between sediments and the

271 granodiorite

At the brecciated contact between the Triassic sediments and the chlorite-quartz bearing

273 vein in contact with the basement (Figure 6a), the Triassic sediments are composed of quartz,

274 chlorite, white mica, Fe-oxides and clasts in a calcite matrix (sample ZAL18-11). The vein is 
composed of mm-size quartz grains recrystallized by bulging and hosting a dense net of fluid

276 inclusions, interlayered with elongate chlorite and white mica ( \pm calcite) (Figure 6b). Along

277 chlorite grain boundaries, abundant Fe-oxides, 20-40 $\mu \mathrm{m}$ large anatase grains, monazite, REE-

278 bearing carbonates and tourmaline are occasionally observed (Figure 6b). The textural aspect

279 of monazite in sediments is similar to that of monazite in the basement (inset in Figure 6b).

280 The red-coloured, foliated pelitic rocks are comprised of white mica, chlorite and quartz

281 grains on the scale of tents of microns ( \pm detrital monazite, apatite and anatase, samples ZAL18-

28212 and ZAL18-13). The veins are approximately $100 \mu \mathrm{m}$ thick, they opened at high angle with

283 the foliation and are filled by elongate grains of quartz rich in fluid inclusions (in the core of 284 the vein). Chlorite developed along the vein-host rock interface with long axes parallel to the 285 vein opening direction (Figure 6c). Veins are stretched and displaced by 30-50 $\mu \mathrm{m}$ secondary 286 chlorite and quartz-bearing layers cross-cutting the foliation, forming 'en échelon' fractures 287 (Figure 6c-d). Monazite in pelites forms euhedral grains 5-10 $\mu \mathrm{m}$ in diameter, sometimes in association with Fe-oxides and xenotime at the interface between the veins and host-rock.

\section{ANALYTICAL AND COMPUTATIONAL METHODS}

291 The chemical composition of metamorphic chlorite and white mica has been analysed with a CAMECA SX-FIVE electron probe micro-analyser (EPMA) at ISTeP (Sorbonne Université). Point measurements were made under a $15 \mathrm{kV}$ acceleration voltage and $10 \mathrm{nA}$ beam current

294 with a $\sim 1 \mu \mathrm{m}$ beam size, in wavelength-dispersive spectroscopy (WDS) mode, using diopside $295(\mathrm{Ca}, \mathrm{Mg}, \mathrm{Si}), \mathrm{MnTiO}_{3}(\mathrm{Mn}, \mathrm{Ti})$, orthoclase $(\mathrm{K}, \mathrm{Al})$, hematite $(\mathrm{Fe})$, albite $(\mathrm{Na})$ and $\mathrm{Cr}_{2} \mathrm{O}_{3}(\mathrm{Cr})$ 296 as standards for measuring the elements written in parentheses. The compositional variability 297 of chlorite and white mica in different microstructural sites was characterised by element mapping using both the EPMA and a Zeiss SUPRA-55VP scanning electron microscope (SEM) at ISTeP (Sorbonne Université). EPMA elemental maps were acquired in WDS mode at 100 
300 nA beam current, $15 \mathrm{keV}$ acceleration voltage with a $200 \mathrm{~ms}$ dwell time. SEM maps were 301 acquired in energy-dispersive spectroscopy (EDS) mode at $15 \mathrm{keV}$ acceleration voltage, $30 \mathrm{nA}$ 302 beam current with a dwell time of $256 \mu$ s. SEM and EPMA maps were quantified with the 303 software XMapTools (Lanari et al., 2014). The U, Th, Pb and REE contents of monazite were 304 analysed with the JEOL-JXA-8230 EPMA at ISTerre (University of Grenoble), at $15 \mathrm{keV}$ 305 acceleration voltage, $200 \mathrm{nA}$ beam current with a spot beam size. For each monazite analysis, 306 a chemical $\mathrm{U}-\mathrm{Th} / \mathrm{Pb}$ age was determined and the average age of different populations of 307 monazite (identified on the base of compositional criteria) was calculated with the software 308 NiLeDAM. The analytical protocol and data processing are detailed in Appendix 1.

Titanite, anatase and zircon were dated in-situ by the U-Pb LA-ICP-MS technique at 310 Trinity College, Dublin (Ireland), on the same thin sections as used for the microstructural and 311 petrological studies. U-Pb ages were determined using a Photon Machines Analyte Exite 193 nm ArF Excimer laser-ablation system with a Helex 2-volume ablation cell coupled to an Agilent 7900 ICPMS. For titanite, zircon and anatase, 300 shots were ablated per analysis with 314 a laser spot size of $24 \mu \mathrm{m}$ (Figure S1). The laser repetition rate and fluency were set at $15 \mathrm{~Hz}$, $3151.60 \mathrm{~J} / \mathrm{cm}^{2}$ and $11 \mathrm{~Hz}$ and $2.25 \mathrm{~J} / \mathrm{cm}^{2}$ for anatase/titanite and zircon respectively, and typically 31650 analyses were obtained on each sample (usually 1-2 spots per grain) and plotted on a Tera317 Wasserburg diagram (Tera and Wasserburg, 1972) with $2 \sigma$ error ellipses, allowing 318 determination of the initial $\mathrm{Pb}$ intercept on the ${ }^{207} \mathrm{~Pb} /{ }^{206} \mathrm{~Pb}$ axis.

The raw titanite and anatase isotope data were reduced using the "VizualAge 320 UcomPbine" data reduction scheme (DRS) of Chew, Petrus, and Kamber, (2014). The 321 "VizualAge" DRS was employed for zircon data reduction. Common $\mathrm{Pb}$ in the titanite and 322 rutile standards was corrected using the ${ }^{207} \mathrm{~Pb}$-based correction method. For unknowns, the 323 initial $\mathrm{Pb}$ in titanite and anatase was determined from the upper intercept (y-intercept on the $324{ }^{207} \mathrm{~Pb} /{ }^{206} \mathrm{~Pb}$ axis) of the discordia line defined by the analyses on Tera-Wasserbutg Concordia. 
325 The age for each population of grains was calculated from the lower intercept of the discordia

326 line on Tera-Wasserburg Concordia. line with the Pb evolution model. Zircon ages were instead 327 estimated by calculating the weighted average of ${ }^{206} \mathrm{~Pb} /{ }^{238} \mathrm{U}$ corrected ages (Table S3). The

328 primary standards employed, the secondary standard ages and further details on the analytical 329 procedure are reported in Supplementary Material S1. Titanite and anatase included in ilmenite 330 were not dated due to their small size. When anatase core relicts were preserved in titanite grains 331 (type-1 Ant), it was rarely possible to ablate separately relicts of anatase and their titanite host 332 due to the small grain size (e.g. Figure S1). In anatase-dominated samples, where the bulk of 333 grain was anatase, these analyses were classified and processed as anatase, in titanite dominated 334 samples with only minor relict anatase the analyses were classifies as titanite. The contribution 335 of relicts of anatase to the calculated age was estimated by comparison with results from 336 samples where the only anatase or titanite only were sampled. In sample where two types of 337 anatase were observed, all anatase data were processed together. The existence of one or two 338 population(s) of anatase based on the data interpretation was compared to microstructural 339 observations

340 Monazite ages were calculated from the EPMA U, Pb and Th contents. The associated 341 detection limits were 90 ppm for $\mathrm{Pb}, 120 \mathrm{ppm}$ for $\mathrm{U}$ and $115 \mathrm{ppm}$ for Th. For each monazite 342 analysis, a chemical U-Th- $\mathrm{Pb}$ age was determined (see Montel et al., 1996). When the $\mathrm{Pb}$ 343 content was lower than the EPMA detection limit, a value of 90 ppm was used to estimate a 344 maximum age.

345 The crystallization temperature and the minimum content of $\mathrm{XFe}^{3+}\left(\mathrm{Fe}^{3+} /\left(\mathrm{Fe}^{2+}+\mathrm{Fe}^{3+}\right)\right)$ 346 of each generation of chlorite in 23 selected samples were estimated using the Chl-Qz- $\mathrm{H}_{2} \mathrm{O}$ 347 thermodynamic model of Vidal et al. (2006) from element abundances at a reference pressure 348 of 3 kbar and $\mathrm{H}_{2} \mathrm{O}$ activity at fixed unit value. The absolute uncertainty on the crystallization 349 temperature estimates is around $50^{\circ} \mathrm{C}$ (Vidal et al., 2006; Dubacq, Soret, Jewison and Agard, 
$3502019)$, although the relative uncertainty between samples is likely lower $\left(10-20^{\circ} \mathrm{C}\right)$. The semi-

351 empirical thermometer of Bourdelle, Parra, Beyssac, Chopin, and Vidal (2013) formulated for 352 low-temperature chlorite was used for comparison.

\section{RESULTS}

\subsection{Composition of pre-kinematic and syn-kinematic chlorite and white mica}

Chlorite generally exhibits compositional variation at the $\mu \mathrm{m}$-scale with high variability in Fe, $\mathrm{Mg}$ and $\mathrm{Al}$ contents (especially in discretely deformed granites), as shown in Figure 7 (and Table S1). In samples collected east of Lake Urdiceto, two groups of chlorite are recognized.

360 The first, named Chl1, is ubiquitous from undeformed granites (pre-kinematic) to mylonites, 361 and mainly observed in ChlA flakes (Figure 7a). It should be noted that Chl1 refers to a chemical composition while ChlA refers to the microtexture of chlorite (which do not necessarily correspond, e.g. Vidal et al., 2006; Airaghi et al., 2017). Chl1 is Al-poor (2.2-2.4 apfu) and Mg-rich (1.7-2.5 apfu). The second group of chlorites, Chl2, is mainly observed in syn-kinematic ChlB (Figure 7a, c-d) and exhibits higher $\mathrm{Al}$ content $(\mathrm{Al}=2.5-2.86 \mathrm{apfu})$ and lower $\mathrm{Mg}$ content $(\mathrm{Mg}=1.5-1.7 \mathrm{apfu})$. The Fe content increases slightly from Chl1 (2.15-2.3 apfu) to Chl2 (2.4-2.5 apfu). In undeformed samples, only homogeneous Chl1 in chlorite flakes is observed (e.g. sample ZA16-84). In the most deformed samples, chlorite flakes (ChlA) exhibit $\mu \mathrm{m}$-scale $\mathrm{Mg}$ and $\mathrm{Al}$ zoning and portions of Chl1 are pseudomorphically replaced by Ch12. Chl1 is also found as relicts in ChlB in fractures, wrapped by Chl2 (Figure 7a). In samples

371 collected west of Lake Urdiceto, Chl1 in flakes is Fe-rich (1.97-2.13 apfu) and Mg-poor (2.18$2.47 \mathrm{apfu})$ compared to $\mathrm{Chl} 2$ in veins and along fractures $(\mathrm{Fe}=1.39-1.50 \mathrm{apfu}, \mathrm{Mg}=2.83-2.95$ zoning within Chl1 flakes is observed miming cleavage planes of the original magmatic crystal 
375 (biotite or amphibole) in discretely deformed samples, while Chl2 within ChlB within fractures

376 is homogeneous (Figure 7b). In mylonites, chlorite shows intermediate compositions (e.g. 377 sample ZA16-96 in Figure 7c) difficult to relate to a specific microtextural sequence due to 378 extensive deformation. In the Triassic sediments, chlorite is Al-enriched compared to chlorite 379 in the basement, but exhibits similar compositional trends (Figure 7c-e). Two groups are 380 recognized in samples ZAL18-13 and ZAL18-12: one is Mg-depleted (1.7-1.97 apfu) and Al381 enriched (2.90-2.92 apfu) and is observed in veins, the other is Mg-enriched (2.18-2.40 apfu) 382 or Al-depleted (2.68-2.78 apfu) and is observed within the matrix (Figure 7c-e). Only the 383 second group of chlorite is observed in sample ZAL18-11 (Table S1). White mica in the granitic basement shows $55-80 \%$ muscovite $(M s)$, pyrophyllite ( $\operatorname{Prl})$ content in the $0-20 \%$ range and up to $\sim 30 \%$ celadonite content ( $\mathrm{Cel}$, Figure $8 \mathrm{a}$ ). White mica in fractures, veins and in the deformed matrix (syn-kinematic) exhibits lower muscovite $(55-70 \%)$ and higher (or similar) celadonite content (20-30\%) than white mica in chlorite flakes (prekinematic, $70-80 \%$ muscovite, $10-20 \%$ celadonite, Figure $8 \mathrm{a})$. The composition of white mica in sedimentary samples is similar to that of white mica from feldspar in basement samples (Figure 8b), but with lower variability. While in samples ZAL18-12 and ZAL18-11 one single group of white mica is observed, in sample ZAL18-13 two groups are identified: white mica in the veins have higher celadonite contents $(18-28 \%)$ than in the matrix (10-18\%).

\subsection{Thermobarometry of low-grade fluid-rock interaction events}

395 Thermodynamic modelling was combined to empirical thermobarometry to distinguish the conditions of crystallisation of the different generations of chlorite and white mica possibly recorded by the restricted range of compositional variations. Crystallisation temperature calculated with the chlorite thermometers of Vidal et al. (2006) and Bourdelle et al. (2013) are 
400 chlorite calculated with the method of Vidal et al. (2006) ranges between 0.1 and 0.55 , which 401 is realistic for low-T chlorite (Masci et al., 2019). The entire iron content was considered 402 divalent for the thermometer of Bourdelle et al. (2013) as recommended in that study. 403 Calculated temperatures for all groups of chlorites are in the same range, with small but 404 systematic differences between Chl1 and Chl2. In samples recording two compositional groups 405 of chlorites (that correspond to pre and syn-kinematic grains), where Fe variations prevail on 406 other element variations (ZA16-82, B11-19, ZA16-78), Chl1 records higher temperatures (220$\left.407320^{\circ} \mathrm{C}\right)$ than $\mathrm{Chl} 2\left(180-250^{\circ} \mathrm{C}\right)$. In samples where dominant chemical variations involve $\mathrm{Al}$ and $\mathrm{Mg}$ (ZA16-126, ZA16-73, B10-32), Chl1 records lower temperatures (170-280 ${ }^{\circ}$ ) than $\mathrm{Ch} 12$ 409 (230-350 ${ }^{\circ} \mathrm{C}$, Figure 8a). In other samples (ZA16-133, B10-31), where two compositional 410 groups of chlorite were observed, calculated temperature cover the entire temperature range 411 estimated for all samples. Undeformed granite samples preserving only Chl1 (e.g. ZA16-79, B11-16, ZA16-117) record the highest range of temperatures $\left(280-350{ }^{\circ} \mathrm{C}\right)$. Crystallisation 413 temperatures calculated for chlorite in mylonites (e.g. ZA16-96, ZA16-98, ZA16-128, ZA16414 97) are intermediate between the Chl1 and Chl2 crystallisation temperatures. Two ranges of 415 temperatures were also obtained for chlorite in sedimentary samples (ZAL18-12). The highest 416 temperatures $\left(240-350^{\circ} \mathrm{C}\right)$ are generally associated with chlorite in veins, while lowest 417 temperatures $\left(180-280^{\circ} \mathrm{C}\right)$ are mostly associated with chlorites in C planes (with variability, 418 especially for samples collected Est and West of the Lake of Urdiceto, Fig. 1c). Only one range 419 of chlorite temperatures $\left(\mathrm{Chl} 2,200-300^{\circ} \mathrm{C}\right)$ is observed within the vein of sample ZAL18-11. 420 To test for the statistical validity of the two temperature intervals $\left(240-350^{\circ} \mathrm{C}\right.$ and $\left.180-280^{\circ} \mathrm{C}\right)$, 4213000 temperatures were randomly sampled from the dataset calculated with the thermometer 422 of Vidal et al. (2006) assuming a Gaussian error of $\pm 30^{\circ} \mathrm{C}$ (Figure 9b). In the basement, two 423 main peaks at 260 and $330^{\circ} \mathrm{C}$ persist. Scattered values at low-T conditions are attributed to 
424 modelling artefacts due to $\mathrm{Fe}^{3+}$ overestimation and/or to departure of some chlorite

425 compositions from equilibrium with assumed unit activity for quartz and water.

426 The moderate celadonite contents of white mica (accounting for the Tschermark substitution

427 only) suggest crystallization pressures in the range 1-4.5 kbar following the calibration of 428 Massone and Schreyer (1988) (Figure 9c), as opposed to sub-surface hydrous alteration of 429 feldspar only, which would favour pyrophyllitic substitution (Dubacq, Vidal, \& De Andrade, 430 2010). Following this calibration, the higher celadonite content (and Si apfu content) of white 431 mica in fractures, veins and shear bands indicates higher pressure conditions than in white mica 432 growing from feldspar or from biotite (white mica in flakes, Figure 9c).

4345.3 In situ $\mathrm{U}-\mathrm{Th} / \mathrm{Pb}$ dating of magmatic zircon and hydrothermal anatase, titanite and 435 monazite

436 Results for titanite, anatase and monazite dating are shown in Figure 10a-c and summarized in 437 Table 1; the raw isotopic ratios and age data for zircon, titanite and anatase are provided in 438 Table S3. The entire geochronological dataset is combined with previous literature age 439 constraints of metamorphic events, derived from other localities of the Axial zone in Figure 440 10d. The composition of monazite together with all concordias for zircon, titanite and anatase 441 are provided in Figure S2. Zircon analyses yield weighted mean Concordia ages at 300-310 Ma 442 (Figure 10d and f). Some zircon analyses, especially in sample ZA16-119, exhibit Pb loss and 443 broadly define a discordia line intercepting the Concordia at c. 285-290 Ma (see Figure S1).

444 Titanite grains contain on average $16 \mathrm{ppm}$ of $\mathrm{U}, 1.7 \mathrm{ppm}$ of Th and $50 \mathrm{ppm}$ of common $445 \mathrm{~Pb}$, and exhibit a $\mathrm{Th} / \mathrm{U}$ ratio of 0.1 with ages spanning from $336 \pm 12$ to $313 \pm 11$ (Figure 10a 446 and d). Titanite in ZA16-84 provides the age constraint with the lowest uncertainty (313 \pm 11 , 447 MSWD of 1.1, Figure 10a) and exhibits the lowest common Pb content (10 ppm) and the highest $448 \mathrm{Th} / \mathrm{U}$ ratio $(0.20)$. Anatase contains on average lower amounts of $\mathrm{U}(8 \mathrm{ppm})$ and common $\mathrm{Pb}$ 
449 (24 ppm), and higher Th contents (3.4 ppm) than titanite, with $\mathrm{Th} / \mathrm{U}$ ratios of 0.4 , with ages

450 from 301 to $48 \mathrm{Ma}$ (Figure $10 \mathrm{~b}$ and d). The age of anatase is difficult to constrain because of 451 high common $\mathrm{Pb}$ and low radiogenic $\mathrm{Pb}$ contents. When possible, two Tera-Wasserburg 452 concordia lower intercepts for anatase $\mathrm{U}-\mathrm{Pb}$ age data were defined for each sample, delimiting 453 the possible youngest and oldest grain populations (e.g. Figure 10b). In this case, youngest ages 454 cluster at c. 30-80 Ma while oldest ages cluster at c. 240-280 Ma (Figure 10b, d). The mylonitic 455 granite ZA16-96 exhibits the young age population only (Tera-Wasserburg concordia lower 456 intercept of $39 \pm 8 \mathrm{Ma}$, Figures $10 \mathrm{c}$ and $\mathrm{S} 1$ ), despite the low variability of the radiogenic $\mathrm{U} / \mathrm{Pb}$ 457 ratio.

Patchy monazite in samples ZA16-98 and ZAL18-11 shows low and variable U and Th contents (Figure 10e and Table S2), typical of low-temperature secondary monazite 460 (Rasmussen \& Muhling, 2007; Janots, Berger, \& Engi, 2011). The U- $\mathrm{Th} / \mathrm{Pb}$ ages (with $\mathrm{Pb}>$ 461 EPMA detection limits of $\sim 100 \mathrm{ppm}$ ) range between 180 and $325 \mathrm{Ma}$ (Figure 10c). In both samples, $\mathrm{Pb}$-depleted domains $(\mathrm{Pb}<$ EPMA detection limits despite Th contents up to few $\mathrm{wt} \%$, Table S2) are observed within Th-depleted monazite grains, indicating late partial replacement 464 of monazite grains by recrystallization and dissolution-reprecipitation (Figure 10e). The oldest 465 ages generally correspond to monazite with relatively high Th content $\left(\mathrm{ThO}_{2}>2.4 \mathrm{wt} \%\right)$ and 466 variable $\mathrm{U}$ content $\left(0.04 \mathrm{wt} \%<\mathrm{UO}_{2}<1.0 \mathrm{wt} \%\right)$. They cluster around $254 \pm 6 \mathrm{Ma}$. A second 467 group of monazite has a lower Th content $\left(\mathrm{ThO}_{2}<1.9 \mathrm{wt} \%\right)$, variable $\mathrm{U}$ content $(0.01 \mathrm{wt} \%<$ $468 \mathrm{UO}_{2}<1.9 \mathrm{wt} \%$ ) and provides an age of $196 \pm 18 \mathrm{Ma}$. In both samples ZA16-98 and ZAL18-11 469 young ages are estimated from the $\mathrm{Pb}$-depleted domains $(\mathrm{Pb}<$ detection limits $)$. Assuming a $\mathrm{Pb}$ 470 content equal to the EPMA detection limit ( $\sim 90 \mathrm{ppm})$, a maximum age for this late stage 471 crystallisation has been calculated at c. $100 \mathrm{Ma}$ in sample ZA16-98 and at c. $20 \mathrm{Ma}$ in sample 472 ZAL18-11 for the domains with the highest $\mathrm{Th}\left(\mathrm{ThO}_{2}=7.6 \mathrm{wt} . \%\right)$ and $\mathrm{U}$ contents $\left(\mathrm{UO}_{2}=0.9750\right.$ 473 wt.\%) (Figure 10c). Cenozoic ages of c. 50 Ma have also been estimated in sample ZA16-98 
474 from thorite-monazite domains. No ages could be obtained for monazite from the sedimentary

475 samples due to their low $\mathrm{U}$, Th and $\mathrm{Pb}$ contents.

\section{6. DISCUSSION}

\subsection{Evaluation of geochronological results}

480 Estimated crystallisation temperatures are far below the closure temperature of $\mathrm{Pb}$ diffusion in 481 rutile $\left(580-630^{\circ} \mathrm{C}\right)$, titanite $\left(575-700^{\circ} \mathrm{C}\right)$, monazite $\left(\sim 800^{\circ} \mathrm{C}\right)$ and zircon $\left(\sim 900^{\circ} \mathrm{C}\right.$, Rubatto, 482 2017; Engi, 2017 and references therein). Therefore, $\mathrm{U}-\mathrm{Th} / \mathrm{Pb}$ dates are interpreted as 483 crystallisation ages. Zircon is dominantly of magmatic origin as attested by its microtextures.

484 Concordant zircon ages, inferred to bracket the timing of the Bielsa pluton emplacement at 300$485310 \mathrm{Ma}$ (Figure 10d and Table 1), are in agreement with previously published ages (Vacherat et al., 2017). Zircon ages therefore constrain the upper limit of the first alteration event.

487 Discordant ages defining a chord with an intercept at c. $290 \mathrm{Ma}$ in sample ZA16-119 (Figure 488 S1) derive from sampling of metamictic zircon domains, characterized by decreasing 489 crystallinity, $\mathrm{Pb}$ loss and variable incorporation of common $\mathrm{Pb}$. Since metamict domains show 490 typical textures of dissolution-reprecipitation under low-T conditions (Geisler et al., 2003) and 491 are observed in undeformed samples, the c. 290 Ma age may indicate re-opening of the system 492 during the early pre-kinematic fluid-rock interaction, started shortly after the pluton 493 emplacement, in Late Variscan times.

Titanite ages are less accurate but cluster within uncertainty of the zircon ages, at c. 300

495 Ma (Late Variscan) (Figure 10d and Table 1). Some of the titanite ages are older than the corresponding zircon ages from the same sample. The titanite Discordia line on Tera-

497 Wasserbourg Concordia diagram is well constrained due to the large variation in $\mathrm{U}: \mathrm{Pb}$ ratio of 498 the titanite grains (Figure 10a and Figure S2). Therefore, the titanite ages marginally older than 
499 their corresponding zircon ages may originate from local incorporation of radiogenic PB at the

500 time of titanite crystallization (see also Essex and Gromet, 2000). The ${ }^{207} \mathrm{~Pb} /{ }^{206} \mathrm{~Pb}$ initial ratio

501 of metamorphic titanite has been shown to vary up to $2 \%$ in the same sample (Kohn, 2017), and

502 is often significantly lower (i.e. more radiogenic) than the crustal evolution model of Stacey

503 and Kramers (1975). This signs the incorporation of radiogenic Pb from the breakdown of a U-

504 rich mineral phase such as rutile or anatase, anatase being observed here as the most common

505 titanite precursor.

The $\mathrm{U} / \mathrm{Pb}$ ages obtained for anatase are less well constrained than the titanite due to

507 higher common $\mathrm{Pb}$, lower radiogenic $\mathrm{Pb}$ contents and the smaller spread in $\mathrm{U}-\mathrm{Pb}$ ratio of the 508 anatase grains. Calculated ages range between $40 \mathrm{Ma}$ and $280 \mathrm{Ma}$. Anatase does not record the 509 magmatic stage since it is observed to replace magmatic ilmenite. If type-1 Ant or titanite 510 recrystallize into type-2 Ant, inter and intra-grain variations in the radiogenic $\mathrm{Pb}$ and $\mathrm{U}$ content 511 could be inherited from the parent mineral given the small transport scales for anatase-forming 512 elements deduced from microtextural evidence (see section 3.1). Such variations and possible 513 incorporation of type-1 Ant in spot ablations of titanite (see section 4) contribute to the large 514 spread of the anatase ages (e.g. samples ZA16-79, ZA16-73). However, a recurrently young 515 signal of c. 40-80 Ma is exclusively observed only in samples with both types of anatase and in 516 mylonites. As solid-state $\mathrm{Pb}$ diffusion can be excluded due to the low metamorphic grade, the 517 youngest $\mathrm{U}-\mathrm{Pb}$ anatase ages likely indicate partial (re-)crystallization of the smallest anatase 518 grains (type-2 Ant), in agreement with microtextural observations. In addition, the upper limit 519 of the range of anatase ages is the youngest in samples with both types of anatase: This suggests 520 mixing at a smaller scale than laser beam size between older titanite-anatase and new anatase. 521 The two age end-members are shown on Figure 10d for all anatase-bearing samples.

522 Monazite is unlikely to record the magmatic stage since (1) magmatic allanite and apatite 523 incorporate the rare earth elements and phosphorous necessary for monazite crystallization, (2) 
monazite is observed to replace magmatic allanite or apatite and (3) the oldest monazite ages

525 are younger than the zircon ages. Monazite ages in both samples ZA16-98 and ZAL18-11

526 indicate a series of events at c. $250 \mathrm{Ma}, 200 \mathrm{Ma}$, and possibly $<50 \mathrm{Ma}$. This range of ages

527 suggests successive episodes of partial dissolution-reprecipitation, as also shown by the patchy

528 textures and the dissolution figures of monazite grains (Figures $5 \mathrm{c}$ and $6 \mathrm{~b}$ ). The mineral

529 assemblage corresponding to the monazite ages of c. 200 Ma remains difficult to identify as

530 textural and chemical evidences is lacking. The ages of 190-250 Ma obtained in monazite of

531 chlorite-bearing veins in sediments indicate that monazite grains were likely inherited from the

532 underlying basement and partially re-crystallized during vein opening and fluid circulation soon

533 after sediments deposition in Triassic times.

534

\subsection{Timing and conditions of fluid-rock interactions and deformation}

Detailed microstructural and petrological observations show a poly-phase alteration history in

537 the Bielsa basement at temperatures below $350^{\circ} \mathrm{C}$. Within this temperature range, a first fluid538 rock interaction event is related to the breakdown of the original magmatic assemblage and the 539 growth of Ttn, Chl1, anatase (type-1 Ant) at $270-350^{\circ} \mathrm{C}$. This alteration event predates all 540 deformation stages since the earliest deformation phase deforms this mineral assemblage and 541 the alteration event is also observed in undeformed samples (e.g. Figure 4a-b). During the first 542 alteration event, the source of $\mathrm{Al}$ and $\mathrm{Ca}$ for chlorite and titanite ( \pm prehnite) growth is the 543 contemporaneous breakdown of plagioclase and the albitization of alkali-feldspar (always 544 largely consumed), irrespective of the strain intensity. Calcium may also derive from external

545 fluids as attested by calcite growth within weakely deformed granite matrix. Titanite, zircon,

546 anatase and oldest monazite ages allow bracketing the timing of the early alteration(s) to 230-

$547300 \mathrm{Ma}$ (late to post-Variscan). This age range is in agreement with ${ }^{40} \mathrm{Ar} /{ }^{39} \mathrm{Ar}$ ages of biotite of 548 c. 280 and 250 Ma obtained in the Bielsa and Maladeta massifs respectively (Jolivet et al., 2007). 
550 during the formation of C-S structures (see section 3) at $\sim 200-280^{\circ} \mathrm{C}$. The pervasive schistosity,

551 kinking, shearing and fracturing are likely to have occurred at the same time since they are

552 kinematically compatible. The S planes are observed in both the basement and in the Triassic

553 sediments (see section 2). The S-forming stage is therefore post-late Triassic in age at least.

In such deformed samples, the youngest ages are yielded by anatase (in samples bearing

both types of anatase) and by monazite; this suggests that deformation triggered the partial recrystallization of anatase and monazite. The youngest anatase ages only (c.40 Ma) and the youngest monazite ages (c. $50 \mathrm{Ma}$ ) are obtained in mylonites (ZA16-96 and ZA16-98). This 558 observation suggests that the mylonitization related to the deformation stage likely occurred 559 between 40 and $70 \mathrm{Ma}$. These ages are in agreement with Rb-Sr ages of c. $48 \mathrm{Ma}$ obtained for the (re)activation of mylonitic bands in the Néouvielle massif $(\sim 15 \mathrm{~km}$ to the northwest of 561 Bielsa, Wayne \& McCaig, 1998). In sample ZA16-79 an age population at c. 120 Ma is 562 observed, but no clear textural relationships are identified, while the large uncertainties mean it 563 Eocene ages. et al. (2013) are consistent despite differences in how they employ the oxidation state of Fe. The calculated range of temperatures $\left(200-350^{\circ} \mathrm{C}\right)$ is also consistent with hydrothermal

567 conditions typically associated with the appearance of prehnite and titanite in the cleavages of 568 biotite (e.g. Eggleton \& Banfield, 1985; Morad, Sirat, El-Ghali, \& Mansurbeg, 2011; Yuguchi et al., 2015) and with temperatures calculated for the overlying sediments, suggesting that fluids were freely circulating between the basement and the overlying sediments. In details,

571 thermometric results indicate that the pre-kinematic greenschist-facies metamorphism occurred 572 at higher temperature $\left(270-350^{\circ} \mathrm{C}\right)$ than later syn-kinematic fluid-rock interaction $\left(200-280^{\circ} \mathrm{C}\right.$, 573 Figure 9b), with spatial variations (east and west of Lake Urdiceto, Figure 9a) due to local 
variations in fluid composition. The systematic differences between temperatures estimated for

575 Chl1 and Chl2 support however the existence of two different fluid-rock interaction events.

The moderate celadonite content of white mica, especially in fractures, veins and shear

577 bands (Figure 8a and c) suggests crystallization at a pressure of 1-5 kbar (1 - $4.5 \mathrm{kbar}$, following

578 the formulation of Massone and Schreyer, 1988, Figure 9c and below $3 \mathrm{kbar}$ according to

579 Cathelineau et al., 1986 for hydrothermal alteration in granites). No paragonite nor margarite components are observed within our white mica dataset. Pyrophyllite substitution, which increases the $\mathrm{Si}$ content of muscovite, is not predominant compared to the Tschermak substitution (Figure 8a). Increasing pyrophyllite content in sample B11-31 implies

583 crystallization during continuous cooling (Dubacq et al., 2010). Fluid inclusion trapping 584 pressures in the range 2-5 kbar are reported by Henderson and McCaig (1996), consistent with pressure estimates from white mica in mylonite veins (Wayne \& McCaig, 1998). The lowest pressure values of $\sim 2$ kbar are consistent with the maximum burial depth estimated for the Bielsa (Jolivet et al., 2007; Bellahsen et al., 2019).

This low-grade petro-chronological dataset allows the timing of low-grade metamorphism and deformation to be discussed in the light of the Variscan and Alpine orogenic cycles, with major implications for the crustal rheology and the evolution of the deformation 591 style in granites.

\subsection{Pre-orogenic alteration and implications for crustal softening prior to shortening}

594 Petro-chronological results and microstructural analyses show that Late to post-Variscan lower

595 greenschist-facies metamorphism in the Bielsa massif transformed an initially strong assemblage made of plagioclase, K-feldspar (forming together close to half of the primary modal composition) and amphibole into weaker, hydrated minerals such as white mica and 
598 chlorite, without associated deformation (as suggested by Bellashen et al., 2019). The

599 breakdown of feldspar to white mica decreases by up to two orders-of-magnitude the sustained

600 shear stress (at a constant strain rate) at $400^{\circ} \mathrm{C}$, lowering the depth of the brittle-ductile

601 transition by at least a third (Wintsch et al., 1995; Gueydan et al., 2003). In the Bielsa massif,

602 pre-orogenic sub to lower greenschist-facies metamorphic reactions have two effects: they

603 enhance grain size reduction (e.g. from magmatic feldspars to minute white micas) and also

604 replace strong minerals such as feldspar and amphibole with mechanically weaker

605 phyllosilicates (e.g. Shea \& Kronenberg, 1993). Both processes are known to result in enhanced

606 softening of the granitic rocks (e.g. Fitz Gerald \& Stünitz, 1993; Wintsch et al., 1995; Oliot,

607 Goncalves, Marquer, 2010). Early pre-kinematic alteration therefore considerably softened the

608 granite at least $150 \mathrm{Ma}$ before the major Alpine shortening phases. Furthermore, the massive

609 growth of pre-kinematic and pre-Alpine white mica and chlorite indicates high fluid-rock ratios

610 during Late-to post-Variscan times and may strongly influence the water availability in the

611 rocks at the grain boundaries during deformation. This has been shown to have major effects

612 on the mechanisms of strain accommodation in other major phases as quartz (e.g. Palazzin et

613 al., 2018) and hence impact the deformation style.

614 Sericitisation of feldspar and chloritisation of biotite is observed to increase with

615 deformation. This suggests that granite alteration continued during syn-kinematic Alpine

616 shortening. In mylonites, syn-kinematic chlorite and white mica partly replace the pre-

617 kinematic chlorite and mica grains, this time without needing huge supplementary amounts of

618 external fluid. Consequently, in the latter fluid-rock interaction event, there is no evidence for 619 additional large-scale reaction-driven softening outside mylonitic zones, consistent with 620 observations reported for the Néouvielle massif ( $15 \mathrm{~km}$ to the northwest, Wayne \& McCaig, 621 1998). The crystallisation of new phyllosilicates and the larger amount of fluid inclusions in 622 quartz in fractured and mylonitic samples as well as the partial age resetting up to 40 Ma close 
623 to shear zones also appear to indicate progressive localisation of metamorphism with strain

624 localisation.

Zones of fractures and faults indicate that granitic rocks were also deformed by frictional

626 behaviour, as commonly observed at temperatures of $200-350^{\circ} \mathrm{C}$ (e.g. Copley, 2017). The

627 crystallization of phyllosilicates during a pre-orogenic alteration event may weaken fault zones

628 by at least $30 \%$ as phyllosilicates have much lower coefficients of friction than undeformed and 629 unaltered crystalline rocks (e.g. Kaduri, Gratier, Renard, Çakir, \& Lasserre, C., 2017; Copley, 630 2017). The pre-existence of phyllosilicate in the Bielsa basement therefore appears to have 631 generated a network of weak rocks with low friction coefficient resulting in their distributed 632 deformation.

\subsection{Effect of the early alteration on deformation patterns at field scale}

635 The pervasive schistosity (S) observed within the Bielsa massif along the studied transects 636 indicates that the deformation is distributed at the scale of the massif(Figure 1b). The existence 637 of mylonitic corridors shows a phase of strain localization with variations in the sense of shear 638 at the kilometric to decametric scale, but their high density also results in a distributed 639 deformation at the massif scale. At Bielsa, both $\mathrm{S}$ and $\mathrm{C}$ forming stages post-date the Late to 640 post-Variscan pervasive alteration that softened the basement. In the Maladeta massif, (50-60

$641 \mathrm{~km}$ to the northeast), less pervasive pre-kinematic alteration is observed as well as localized 642 deformation and lower density of mylonitic corridors (Leblanc, Gleizes, Lespinasse, Olivier, \& 643 Bouchez, 1994). This indicates that pre-orogenic crustal softening controlled the deformation 644 patterns observed on a outcrop scale, in particular the frequency of mylonitic bands the location 645 of faults and of fractures. 
648 young ages from the mylonites. During this stage, the re-activation of steep faults may have 649 served as fluid pathways and triggered alteration. Interfaces between differently-altered 650 domains formed different phase strength contrasts. Consequently, ductile strain and reactions 651 interacted positively to produce narrow ductile shear zones (as described by Holyoke \& Tullis, 652 2006). Furthermore, deformation at this stage may strongly influence the breakdown on residual 653 feldspar to muscovite (e.g. Knipe \& Wintsch, 1985), as attested by the greater amount of white 654 mica observed in mylonites compared to weakly and undeformed samples. The youngest ages obtained in this study (c. $40 \mathrm{Ma}$ ) are older than the activation of the

656 Gavarnie and Guarga thrusts (Figure 1b), in the hanging and footwall of the Bielsa unit, dated 657 at 32-36 Ma et 20-30 Ma respectively (e.g. Bosch et al., 2016; Elmola et al., 2018) and similar 658 to the age of mylonitization in the Néouvielle massif (Wayne \& McCaig, 1998). This may 659 indicate that the distributed shortening in Bielsa and at a larger scale predates the localization 660 of the strain along some of the major thrust of the Axial zone. These findings are consistent 661 with the distributed / localized modes of shortening documented in the external Western Alps 662 (Bellahsen et al., 2014), in the central Alps (Wehrens, Baumberger, Berger \& Herweg, 2017) 663 and in the Longmen Shan where pre-kinematic and a syn-kinematic greenschist-facies 664 metamorphic events were also recognized (Airaghi et al., 2017b), indicating that they might be 665 typical of the evolution of basement massif in the external domains of the orogenic wedges.

\subsection{Onset and preservation of early alteration in granitic basement}

668 Late to post-Variscan fluid-rock interaction took place on the scale of the Axial zone as metamorphic reactions similar to those observed in the Bielsa massif are found in other crystalline massifs in the Pyrenees (e.g. Maladeta, Néouvielle, Col de Jau; Cathelineau, 1986;

671 Boutin et al., 2016). The early alteration stages may be linked to protracted magmatic activity 672 recorded in the Pyrenean domain throughout the entire Permian (Denèle, Paquette, Olivier, \& 673 Barbey, 2012). Late Variscan magmatic activity has also been invoked as the cause of 
674 hydrothermal alteration in the French Massif Central (Cathelineau, 1986; Turpin, Leroy, \&

675 Sheppard, 1990). Permian to Triassic ages similar to those obtained for fluid-rock interaction events in the Bielsa massif have also been documented in the Variscan basement of the Eastern

677 Iberian Central System and in NE Spain by fluid inclusion and isotopic studies (Tornos, 678 Delgado, Casquet, \& Galindo, 2000 and references therein). The associated hydrothermal 679 alteration in these cases is related to hydrothermal cells formed during extensional tectonic 680 events rather than to plutonism (Tornos et al., 2000; Cardellach, Canals, \& Grandia, 2002). In 681 the Bielsa massif, high fluid-rock ratios have been proposed to result from ingress of seawater 682 and meteoric fluids during the extensional phase following the Variscan orogeny (McCaig, 683 Tritlla, \& Banks, 2000). This is consistent with the fact that the Bielsa basement was exposed 684 at the surface before or during the Permian, as attested by the erosional unconformity between 685 the basement and its sedimentary cover. The preservation of early alteration features in granites over two orogenic cycles (Variscan and Alpine) implies that the basement remained at low grade conditions for over 200 Ma. In the Pyrenees, the main post-Variscan metamorphic phase related to fluid circulation is dated at $110 \mathrm{Ma}$ (e.g. Boutin, 2016). Although Cretaceous ages were obtained for samples which contain both types of anatase, the large $\mathrm{U} / \mathrm{Pb}$ age uncertainties

690 for this time period in our petro-chronological dataset mean that strong evidence for extensive 691 fluid circulation, mineral replacement and deformation during Cretaceous times is lacking. This 692 is in contrast to the eastern Pyrenees where a hydrothermal event characterized by newly formed 693 rutile and titanite associated with Cretaceous rifting overprints the 'post'-Variscan 694 hydrothermal alteration assemblages (Figure 10d; Boutin et al., 2016; Fallourd et al., 2014). 695 This may indicate that, within the Pyrenean belt, metamorphism related to rifting has only 696 weakly affected the southern part of the Axial zone. 
699 The granitic basement rocks in the southern Axial zone of the Pyrenees (Bielsa massif) acted 700 as reactive rocks at low temperatures $\left(\mathrm{T}<350{ }^{\circ} \mathrm{C}\right)$ over a long $(c .300 \mathrm{Ma})$ polyphase history. 701 At an outcrop scale, the Bielsa pluton and the Triassic sedimentary cover exhibit a pervasive 702 N-dipping schistosity, folded or associated to steep C planes localized within a dense network 703 of mylonitic bands. Within the granitic basement, partial mineral replacement and 704 compositional variations at the micron-scale record an extensive fluid-rock interaction event at $705 \sim 270-350^{\circ} \mathrm{C}$ in Late to post-Variscan times (230-300 Ma), as indicated by the titanite, anatase 706 and zircon $\mathrm{U} / \mathrm{Pb}$ ages dataset. This event is ubiquitous and particularly well preserved in the 707 less deformed areas of the massif. It is responsible for pre-kinematic (pre-Alpine) plagioclase 708 and amphibole replacement with growth of phyllosilicates, some tens of million years after 709 pluton emplacement. A second syn-kinematic fluid-rock interaction event is marked by the 710 (re)crystallisation of phyllosilicates at $\sim 200-280^{\circ} \mathrm{C}$ and is related to the formation of mylonitic 711 corridors and fractures parallel to the $\mathrm{C}$ shear planes. In the most deformed samples, despite the 712 low radiogenic $\mathrm{Pb}$ content, monazite and anatase $\mathrm{U}-\mathrm{Th} / \mathrm{Pb}$ ages systematically show a young 713 age component at 40-70 Ma, coeval with the Alpine/ Pyrenean deformation in the southern 714 Axial zone and related to the second alteration event. As the strength of phyllosilicate-rich 715 material is lower by up to an order-of-magnitude than that of the original granite, the granitic massif was significantly softened (and hydrated) at least 150-200 Ma before the major Alpine

717 shortening phase. The dense network of mylonites in the Bielsa pluton resulted in distributed 718 deformation at the massif scale before the localization of the strain along the major thrusting 719 systems of the Axial zone. Pre-orogenic crustal softening controlled the deformation patterns 720 where strain and shortening localized during successive deformation events. Pre-orogenic 721 greenschist-facies metamorphism is often poorly characterized compared to higher-grade 722 metamorphic domains in mountain belts. The case of the Bielsa massif shows that moderate 723 analytical effort can provide valuable information allowing for a better constraints on the 
724 rheological and thermobarometric evolution of basement rocks during the orogenic cycle, even

725 at low-grade conditions.

\section{ACKNOWLEDGEMENTS}

728 This study was founded by the CNRS-BRGM-TOTAL 'OROGEN' project. We acknowledge

729 Prof. S. Duchene and the anonymous review for their constructive comments and remarks.

730 We thank Michel Fialin, Nicolas Rividi and Omar Boudouma for their help with the electron 731 microscopy at ISTeP. DC acknowledges support from Science Foundation Ireland grants 732 12/IP/1663, 15/IA/3024 and 13/RC/2092 (iCRAG Research Centre). iCRAG is funded under 733 the SFI Research Centres Programme and is co-funded under the European Regional

734 Development Fund. We also thank Gary O'Sullivan (Trinity College) for his support with the 735 LA-ICP-MS analysis. Authors have no conflict of interest to declare.

\section{REFERENCES}

738 Airaghi, L., Janots, E., Lanari, P., de Sigoyer, J. \& Magnin, V. (2018). Allanite petrochronology in fresh and retrogressed garnet-biotite metapelites from the Longmen Shan (eastern Tibet), Journal of Petrology, in press.

741 Airaghi, L., de Sigoyer, J., Lanari, P., Guillot, S., Vidal, O., Monié, P., ... Tan, Xibin (2017). Total exhumation across the Beichuan fault in the Longmen Shan (eastern Tibetan plateau, China) : Constraints from petrology and thermobarometry. Journal of Asian Earth Sciences, 140, 108-121.

Bellahsen, N., Mouthereau, F., Boutoux, A., Bellanger, M., Lacombe, O., Jolivet, L., ... Rolland, Y. (2014). Collision kinematics in the western external Alps, Tectonics, 33. 
Bellahsen, N., Bayet, L., Denele, Y., Waldner, M., Airaghi, L., Rosenberg, C. ... Vacherat, A. (2019). Shortening of the axial zone, Pyrenees: Shortening sequence, upper crustal mylonties and crustal strength. Tectonophysics, 766, 433-452.

Bosch, G. V., Teixell, A., Jolivet, M., Labaume, P., Stockli, D., Domènech, M., ...Monié, P. (2016). Timing of Eocene-Miocene thrust activity in the Western Axial Zone and Chaînons Bérnais (west-central Pyrenees) revealed by multi-method thermochronology. Comptes Rendus Geoscience, 348(3-4), 246-256.

Bourdelle, F., Parra, T., Beyssac, O., Chopin, C. \& Vidal, O. (2013). Clay minerals as geothermometer: A comparative study based on high spatial resolution analyses of illite and chlorite in Gulf Coast sandstone (Texas, U. S.A.). American Mineralogist, 98, $914-$ 926.

Boutin, A., de Saint Blanquat, M., Poujol, M., Boulvais, P., de Parseval, P., Rouleau, 759 C., ...Robert, J.-F. (2016). Succession of Permian and Mesozoic metasomatic events in the eastern Pyrenees with emphasis on the Trimouns talc-chlorite deposit. International Journal of Earth Science, 105, 747-770.

Cardellach, E., Canals, A. \& Grandia, F. (2002). Recurrent hydrothermal activity induced by 763 successive extensional episodes: the case of the Berta $\mathrm{F}-(\mathrm{Pb}-\mathrm{Zn})$ vein system $(\mathrm{NE}$ Spain). Ore Geology Reviews, 22, 133-141.

Casas, A.M., Oliva, B., Román-Berdiel, T. and Pueyo, E. (2003). Basement deformation: tertiary folding and fracturing of the Variscan Bielsa granite (Axial zone, central Pyrenees). Geodinamica Acta, 16(2-6), 99-117.

Cathelineau, M. (1986). The Hydrothermal Alkali Metasomatism Effects on Granitic Rocks : Quartz Dissolution and Related Subsolidus Changes. Journal of Petrology, 27, 945-965. mineral standards with variable common $\mathrm{Pb}$. Chemical Geology, 363, 185-199. 
772 Cochelin, B., Lemirre, B., Denèle, Y., de Saint Blanquant, M., Lahfid, A., \& Duchene, S.

773

774

775

776

777

778

779

780

781

782

783

784

785

786

787

788

789

790

791

792

793

794

795 (2017). Structural inheritance in the Central Pyrenees: the Variscan to Alpine tectonometamorphic evolution of the Axial Zone. Journal of the Geological Society, $175(2), 336$.

Copley, A. (2017). The strength of earthquake-generating faults. Journal of the Geological Society, 10.1144/jgs2017-037.

Denèle, Y., Paquette, J.-L., Olivier, P. \& Barbey, P. (2012). Permian granites in the Pyrenees: the Aya pluton (Basque Country). Terra Nova, 24, 105-113.

Dubacq, B., Soret, M., Jewison, E., Agard, P. (2019). Early subduction dynamics recorded by the metamorphic sole of the Mt Albert ophiolitic complex (Gaspé, Quebec). Lithos, 334 - 335, 161 - 179, doi: 10.1016/j.lithos.2019.03.019.

Dubacq, B., Vidal, O., De Andrade, V., 2010. Dehydration of dioctahedral aluminous phyllosilicates: thermodynamic modelling and implications for thermobarometric estimates. Contributions to Mineralogy and Petrology 159, 159-174.

Eggleton, R. A. \& Banfield, J., F. (1985). The alteration of granitic biotite to chlorite. American Mineralogist, 70, 902-910.

Elmola, A. A., Buatier, M., Monié, P., Labaume, P., Trap, P., \& Charpentier, D. (2018). 40Ar/39Ar muscovite dating of thrust activity: a case study from the Axial Zone of the Pyrenees. Tectonophysics, in press.

Engi, M. (2017). Petrochronology Based on REE-Minerals: Monazite, Allanite, Xenotime, Apatite. In: Petrochronology: Methods and Applications, Kohn, M. J., Engi, M. \& Lanari, P. Reviews in Mineralogy \& Geochemistry.

Fallourd, S., Poujol, M., Boulvais, P., Paquette, J.-L., de Saint Blanquant, M., \& Rémy, P. (2014). In situ LA-ICP-MS U-Pb titanite dating of Na-Ca metasomatism in orogenic 
belts: the North Pyrenean example. International Journal of Earth Science, 103, 667682.

Fitz Gerald, J. D. \& Stünitz, H. (1993). Deformation of granitoids at low metamorphic grade. I: Reaction and grain size reduction. Tectonophysics, 221 (3-4), 269-297.

Geisler, T., Rashwan, A. A., Rahn, M. K., Poller, U., Zwingmann, H., Pidgeon, R. 801 T., ...Tomaschek, F. (2003). Low-temperature hydrothermal alteration of natural metamict zircon from the Eastern Desert, Egypt. Mineralogical Megazine, 67(3), 485508.

Goncalves, P., Oliot, E., Marquer, D. \& Connolly, J. A. D. (2012). Role of chemical processes on shear zone formation: an example from the Grimsel metagranodiorite (Aar massif, Central Alps). Journal of Metamorphic Geology, 30(7), 703-722.

Grand'Homme, A., Janots, E., Seydoux-Guillaume, A.M., Guillaume, D., Magnin, V., 808 Hövelmann, J., ...Boiron, M.C. (2018) Mass transport and fractionation during monazite alteration by anisotropic replacement. Chemical Geology, 484, 51-68.

Gueydan, F., Leroy, Y.M., Jolivet, L., \& Agard, P. (2003). Analysis of continental midcrustal strain localization induced by microfracturing and reaction-softening. Journal of

813 Henderson, I. H. C. \& McCaig, A.M. (1996). Fluid pressure and salinity variations in shear zone-related veins, central Pyrenees, France: Implications for the fault-valve model. Tectonophysics, 262, 321-348.

Hansen, E., Reimink, J. \& Harlov, D. (2010). Titaniferous accessory minerals in very low-grade metamorphic rocks, Keweenaw Peninsula Michigan, USA. Lithos, 116, 167-174. shear zone in a granodiorite under greenschist facies conditions, Néouvielle massif, Pyrenees, France. Journal of Structural Geology, 21, 555-576. 
Jackson, J. A., Austrheim, H., McKenzie, D. \& Priestley, K. (2004). Metastability, mechanical strength, and support of mountain belts. Geology, 32(7), 625-628.

Janots, E., Berger, A. \& Engi, M. (2011). Physico-chemical control on the REE minerals in chloritoid-grade metasediments from a single outcrop (Central Alps, Switzerland). Lithos, 121, 1-11.

Jolivet, M., Labaume, P., Monié, P., Brunel, M., Arnaud, N., \& Campani, M. (2007). Thermochronology constraints for the propagation sequence of the south Pyrenean basement thrust system (France-Spain). Tectonics, 26, TC5007.

Kaduri, M., Gratier, J.-P., Renard, F., Çakir, Z., \& Lasserre, C. (2017). The implications of fault zone transformation on aseismic creep: Example of the North Anatolian Fault, Turkey, Journal of Geophysical Research : Solid Earth, 122, 4208-4236.

Knipe, R. J. \& Wintsch, R. P. (1985). Heterogeneous, deformation, foliation development, and metamorphic processes in a polyphaser mylonite. In: Thompson, A. B., Rubie, D. C. (Eds.), Advances in Physical Chemistry, 4, Springer-Verlag, New York, 180-210.

Kohn, M. J. (2017). Titanite petrochronology, In: Petrochronology: Methods and Applications, Kohn, M. J., Engi, M. \& Lanari, P. Reviews in Mineralogy \& Geochemistry.

Lanari, P., Vidal, O., De Andrade, V., Dubacq, B., Lewin, E., Grosch, E., ...Schwartz, S. (2014). XMapTools: a MATLAB-based program for electron microprobe X-ray image processing and geothermobarometry. Computers \& Geosciences 62, 227-240.

Leblanc, D., Gleizes, G., Lespinasse, P., Olivier, Ph. \& Bouchez, J.-L. (1994). The Maladeta granite polydiapir, Spanish Pyrenees : a detailed magneto-structural study. Journal of Structural Geology, 16(2), 223-235.

Mansard, N., Raimbourg, H., Augier, R., Précigout, J. \& Le Breton, N. (2018). Large-scale strain localization induced by phase nucleation in mid-crustal granitoids of the south Armorican massif. Tectonophysics, 745, 46-65. 
846 Masci, L., Dubacq, B., Verlaguet, A., Chopin, C., De Andrade, V., \& Herviou, C. (2019). A 847 XANES and EPMA study of $\mathrm{Fe}^{3+}$ in chlorite: importance of oxychlorite and implications for cation site distribution and thermobarometry. American Mineralogist. 104(3), 403-417.

850 Massonne, H-J. \& Schreyer, W. (1987). Phengite geobarometry based on the limiting assemblage with K-feldspar, phlogopite, and quartz. Contributions to Mineralogy and Petrology, 96, 2, 212-224, doi: 10.1007/BF00375235.

853

McCaig, A. M., Tritlla, J. \& Banks, D.A. (2000). Fluid mixing and recycling during Pyrenean thrusting: evidence from fluid inclusion halogen ratios. Geochimica et Cosmochimica Acta, 64,(19), 3395-3412.

Morad, S., Sirat, M., El-Ghali, M. A., K. \& Mansurbeg, H. (2011). Chloritization in Proterozoic granite from the Äspö Laboratory, southeastern Sweden: record of hydrothermal alterations and implications for nuclear waste storage.Clay Minerals, 46, 495-513.

Oliot, E., Goncalves, P. \& Marquer, D. (2010). Role of plagioclase and reaction softening in metagranite shear zone at mid-crustal conditions (Gotthard Massif, Swiss Central Alps). Journal of Metamorphic Geology, 28, 849-871.

862 Palazzin, G., Raimbourg, H., Stünitz, H., Heilbronner, R., Neufald, K., \& Précigout, J. Evolution in $\mathrm{H}_{2} \mathrm{O}$ contents during deformation of polycrystalline quartz: An experimental study. Journal of Structural geology, 114, 95-110.

865 Parneix, J. C., Beaufort, D., Dudoignon, P. \& Meunier, A. (1985). Biotite chloritization process in hydrothermally altered granites. Chemical Geology, 51, 89-101.

867 Paquette, J.-L., Gleizes, G., Leblanc, D. \& Bouchez, J.-L. (1997). Le granite de Bassiès 868 (pyrénées) : un pluton syntectonique d'âge Westphalien. Géochronologie U-Pb sur zircons. Compte Rendus de l'Académie des Sciences de Paris 324, 387-392. 
870 Rasmussen, B. \& Muhlig, J. R. (2007). Monazite begets monazite: evidence for dissolution of detrital monazite and reprecipitation of syntectonic monazite during low-grade regional metamorphism. Contribution t Mineralogy and Petrology, 154, 675-689.

873

Román-Berdiel, T., Casas, A. M., Oliva-Urcia, B., Pueyo, E. L. \& Rillo, C. (2004). The main Variscan deformation event in the Pyrenees: new data from the structural study of the Bielsa granite. Journal of Structural Geology, 26, 659-677.

Rubatto, D. (2017). Zircon: The Metamorphic Mienral, In: Petrochronology: Methods and Applications, Kohn, M. J., Engi, M. \& Lanari, P. Reviews in Mineralogy \& Geochemistry.

Shea, W. T.J., Kronenberg, A.K. (1993). Strength and anisotropy of foliated rocks with varied mica contents. Journal of Structural Geology, 15, 1097-1121.

Stacey, J. S. \& Kramers, J. D. (1975). Approximation of terrestrial lead isotope evolution by two-stage model. Earth and Planetary Science Letters, 26, 207-221.

Tera, F. \& Wasserburg, G. J. (1972). U-Th-Pb systematics in lunar highland samples from the Luna 20 and Apollo 16 missions. Earth and Planetary Science Letters, 17, 36-51.

Tornos, F., Delgado, A. Casquet, C. \& Galindo, C. (2000). 300 Million years of episodic hydrothermal activity: stable isotope evidence from hydrothermal rocks of the Eastern Iberian Central System. Mineralium Deposita, 35, 551-569.

Tullis, J., Yund, R. \& Farver, J. (1996). Deformation-enhanced fluid distribution in feldspar aggregates and implications for ductile shear zones. Geology, 24(1), 63-66.

Turpin, L., Leroy, J. L. \& Sheppard, S. M. F. (1990). Isotopic systematics (O, H, C, Sr, Nd) of superimposed barren and U-bearing hydrothermal system in a Hercynian granite, Massif Central, France. Chemical Geology, 88, 85-98.

Vacherat, A., Mouthereau, F., Pik, R., Huyghe, D., Paquette, J-L., Christophoul, F., ...Tibari, B. (2017). Rift-to-collision sediment routing in the Pyrenees: A synthesis from 
sedimentological, geochronological and kinematic constraints. Earth-Sciences Reviews,

896

897

898

899

900

901

902

903

904

905

906

907

908

909

910

911

912

913

914

\section{SUPPORTING INFORMATION}

916 Additional Supporting Information may be found online in the supporting information tab for

917 this article.

918 Description Supinfo.doc/x

919 Appendix S1. Analytical methods for geochronology and monazite analysis 
920 Figure S1. Example of location of laser spot for zircon, anatase and titanite dating.

921 Figure S2. Tera-Wasserburg diagrams for all analysed anatase, titanite and zircon. Concordia922 discordia diagrams for zircon.

923 Dataset.xls/x

924 Table S1. Rapresentative chemical composition of chlorite and white mica.

925 Table S2. Representative chemical composition of monazite in samples ZA16-98 and ZAL1892611.

927 Table S3. Original istopic data for zircon, titanite and anatase. Graphs represents weighted 928 mean zircon ages.

929

930 FIGURE CAPTION

931 Figure 1 Geological maps and cross section. (a) Schematic structural map of the Pyrenees, 932 modified from Román-Berdiel, Casas, Oliva-Urcia, Pueyo, and Rillo, 2004. Red square: Bielsa, 933 N: Néouvielle, M: Maladeta, J: Jau. (b) Schematic structural cross section of the Axial Zone 934 (modified from Jolivet et al., 2007). Yellow stars indicate limits of the area where pervasive 935 alteration of the original granite has been documented. Black rectangle denotes the studied area.

936 (c) Geological map of the studied area. (d) Cross sections along transects AA' and BB'.

937

938 Figure 2 Field structures around Lake Urdiceto. (a) Mylonitic corridor along the transect BB' 939 of Figure 1c. White: schistosity (S), yellow: shear planes (C). (b) Second example of mylonitic 940 corridor a few hundred meters eastward of transect BB', at the contact with the Triassic 941 sediments. Schistosity (S, filled white lines) is subparallel to S-dipping shear planes (C, marked 942 in yellow) due to high strain. (c) Mylonitic bands within the basement with C planes varying 943 from S-dipping to N-dipping. (d) N-dipping shear planes in a mylonite at the contact between 
944 the basement and the sedimentary cover. (e) Enlarged view of mylonitic granite (see panel a for

945 location). (f) Enlarged view of weakly deformed (but altered) granite (see panel a for location).

946

947 Figure 3 Field structures in the Triassic sediments. (a) Unconformity between the basement

948 and the Triassic sediments. (b) Chlorite-quartz bearing veins at the contact between the

949 basement and the basal breccia in the Triassic sediments. (c) Two generations of chlorite-quartz

950 bearing veins within Triassic sandstones and pelites.

951

952 Figure 4 Photomicrographs (a, c, e and g) and backscattered electron images (BSE) (panels b,

$953 \mathrm{~d}, \mathrm{f}$ and $\mathrm{h}$ ) of granitic basement with increasing deformation. Abbreviations are from Whitney

954 \& Evans (2010), except Wm: white mica; (a) Undeformed but altered granite; (b) BSE image

955 of a Chl + Wm + Ttn pseudomorph after Bt; (c) weakly deformed and altered granite with dark

956 Chl-Wm pseudomorphs after Bt folded to form kink bands; (d) BSE images of a kinked Chl

957 flake; (e) weakly deformed sample with opening fractures. Chlorite flakes are cross cut by

958 secondary mm-size fractures. (f) BSE image of a Chl + Ttn flake after Bt (Bt relicts still visible),

959 cross cut by a fracture filled with secondary chlorite; (g) mylonite; (h) folded Chl $+\mathrm{Wm}+$ Ant

960 flake cross cut by a secondary fracture in a highly deformed sample. Two types of anatase grains

961 are recognized.

962

963 Figure 5 Backscattered images of accessory minerals in the granitic basement. (a) and (b)

964 magmatic Ilm breaking down to Ant + Cal, then to Ttn. (c) Anatase 1 folded within a Chl flake

965 and replaced by Ttn. (d) Magmatic zircon (core) with metamict rims (low CL intensity) in

966 sample ZA16-119. (e) Chlorite flakes (ChlA) with type-1 Ant cross cut by ChlB-bearing

967 fractures with type-2 Ant (sample ZA16-73). (f) Type-2 Ant rimming titanite after anatase 
(type-1 Ant, preserved in the core of titanite). (g) Chlorite flake (ChlA) in sample ZA16-79

with both types of anatase. (h) Relationships between titanite and anatase in sample ZA16-128.

(i) Monazite in sample ZA16-98. The inset image shows the patchy and porous aspect of the

971 monazite grains. (j) Monazite in reaction halos around fractures in sample ZA16-88.

973 Figure 6 Macro- and micro-scale petrography of the Triassic sediments. (a) Hand sample

974 collected at the boundary between sediments and the granitic basement (ZAL18-11). (b)

975 Backscattered electron image of panel a, showing the occurrence of anatase, Fe-oxides and Mnz

976 within neocrystallized $\mathrm{Chl}$ and $\mathrm{Wm}$. The inset show the patchy texture of monazite grains. (c)

977 Chlorite-bearing veins and shear planes forming 'en-echelon' microstructures in sample ZA16-

978 12. (d) Two generations of chlorite (in veins and shear planes) in samples ZA16-12 (zoom of

979 panel c). ChlA and ChlB refer to chlorite flakes and chlorite in fractures/veins respectively but may be different in origin to ChlA and ChlB observed in the basement.

982 Figure 7 Chemical composition of chlorite. (a) Quantified SEM map of $\mathrm{Al}_{2} \mathrm{O}_{3}$ content in 983 chlorite in sample B10-31 (mylonite). (b) Quantified SEM map of FeO content in chlorite of 984 sample ZA16-82 (fractured sample). (c) Elementary Al vs Mg (\%) content of all analysed 985 chlorites; (d) Trends and compositional groups identified in chlorite in samples collected east of Lake Urdiceto; (e) Trends and compositional groups identified in chlorite in samples collected west of Lake Urdiceto.

988

989 Figure 8 Chemical composition of white mica. (a) Compositional triangular plot and trends for 990 white mica in the basement. (b) Compositional ternary diagram of white mica in the 991 sedimentary samples. Mus: muscovite, Cel: celadonite, Pyr: pyrophillite. 
993 Figure 9 Thermobarometric results. (a) Chlorite temperatures calculated with the method of

994 Vidal et al. (2006) (circles) and Bourdelle et al. (2013) (rectangles). (b) Monte Carlo simulation 995 for the temperature dataset calculated for basement chlorite (3000 iterations, see the text for 996 details). (c) Pressure range estimated from the celadonite content in white mica, following the 997 calibration of Massonne and Schreyer (1988). This calibration does not consider the pyrophillite 998 content in white mica, as it is the case in the Bielsa granite. Pressure values should therefore be 999 considered as minimum values.

1000

1001 Figure 10 Geochronology results. (a) Representative Tera-Wasserburg concordia lower 1002 intercept age defined by titanite analyses in an undeformed sample (ZA16-84). (b) Tera1003 Wasserburg Concordia lower intercept ages defined by various anatase populations (red: old, 1004 blue: young) in a deformed sample (ZA16-128). (c) Frequency distribution of monazite ages. 1005 Note that youngest monazite ages are estimated from $\mathrm{Pb}$ depleted domains $(\mathrm{Pb}<\mathrm{EPMA}$ 1006 detection limits of $\sim 100 \mathrm{ppm}$ ) and monazite-thorite domains (see the text for details). Note the 1007 ages calculated for domains where $\mathrm{Pb}$ is below the EPMA detection limit are maximum ages. 1008 (d) Summary of geochronological results (this study) integrated with previously published data 1009 for metamorphic events in the Axial zone. U-Pb Ant-Ttn indicate the ages obtained for analysis 1010 where a titanite locally preserved anatase cores (type-1 Ant, see the text for details). (e) U and 1011 Th content of monazite in sample ZAL18-11. (f) Probability density plot of all zircon ages for

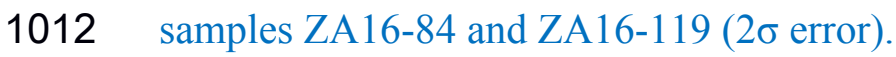

1014 Table 1 Summary of sample descriptions and ages. 


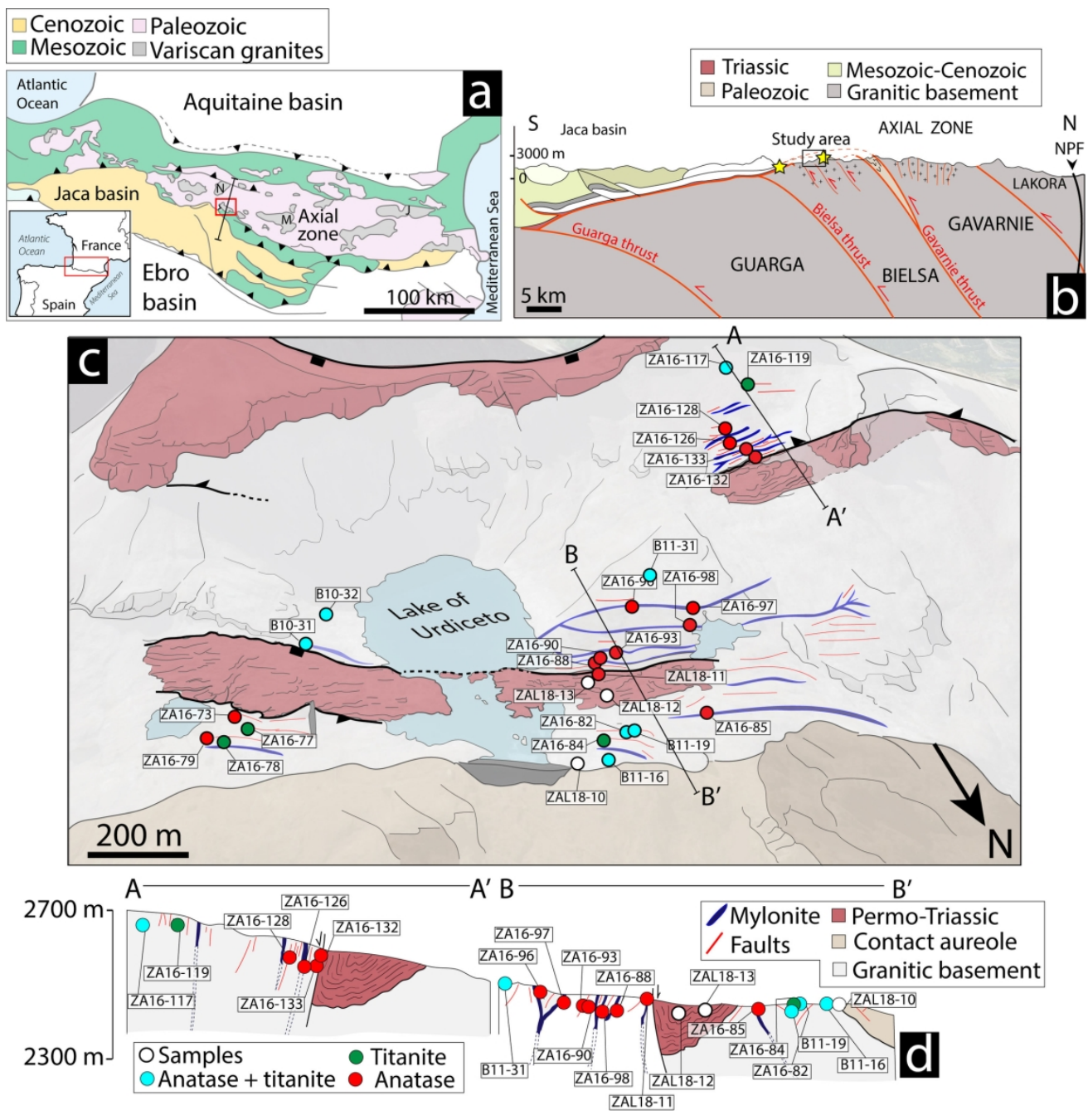

Figure 1. Geological maps and cross section of the studied area

$185 \times 189 \mathrm{~mm}(300 \times 300 \mathrm{DPI})$ 

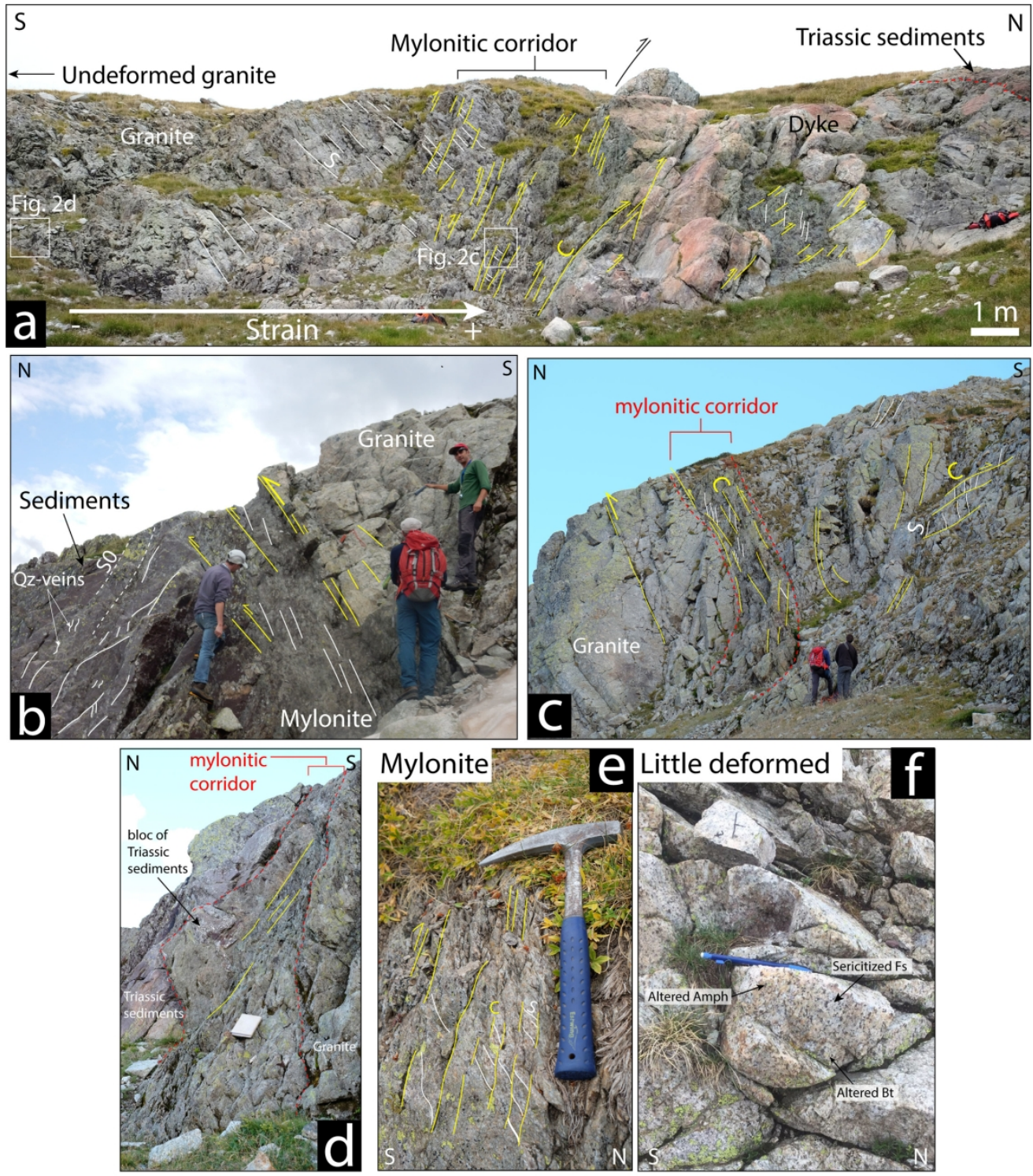

Figure 2. Field relationships within the cristalline basement $180 \times 207 \mathrm{~mm}(300 \times 300 \mathrm{DPI})$ 


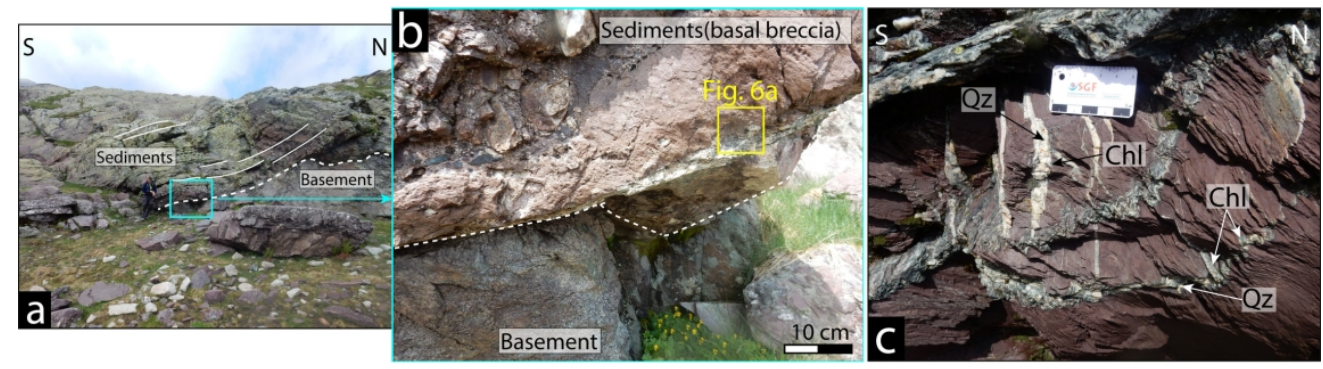

Figure 3. Field relationships within the Triassic sediments $190 \times 53 \mathrm{~mm}(300 \times 300 \mathrm{DPI})$ 


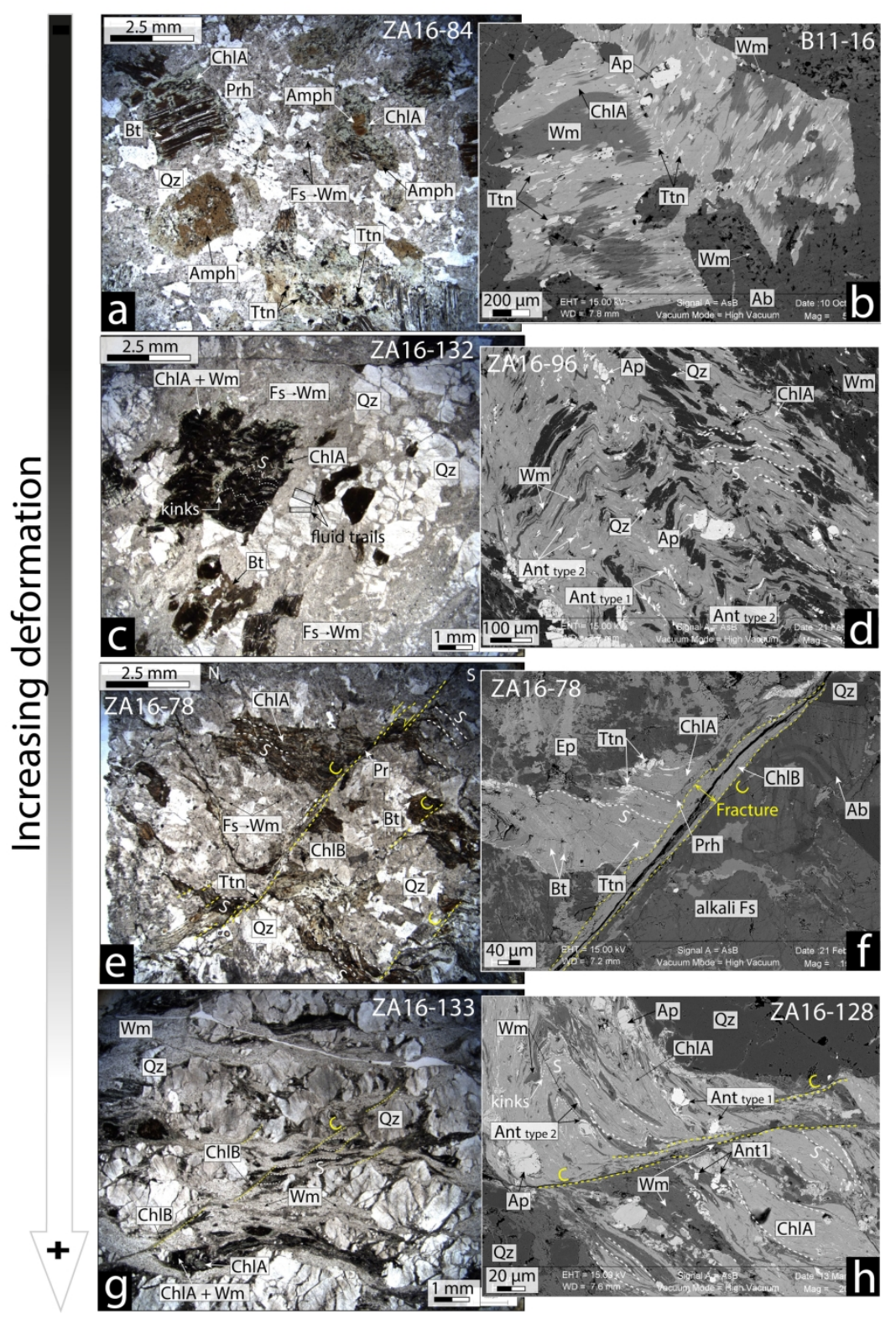

Figure 4. Microphotographs (optical microscope and SEM) of differently deformed granodioritic samples $204 \times 305 \mathrm{~mm}(300 \times 300$ DPI) 

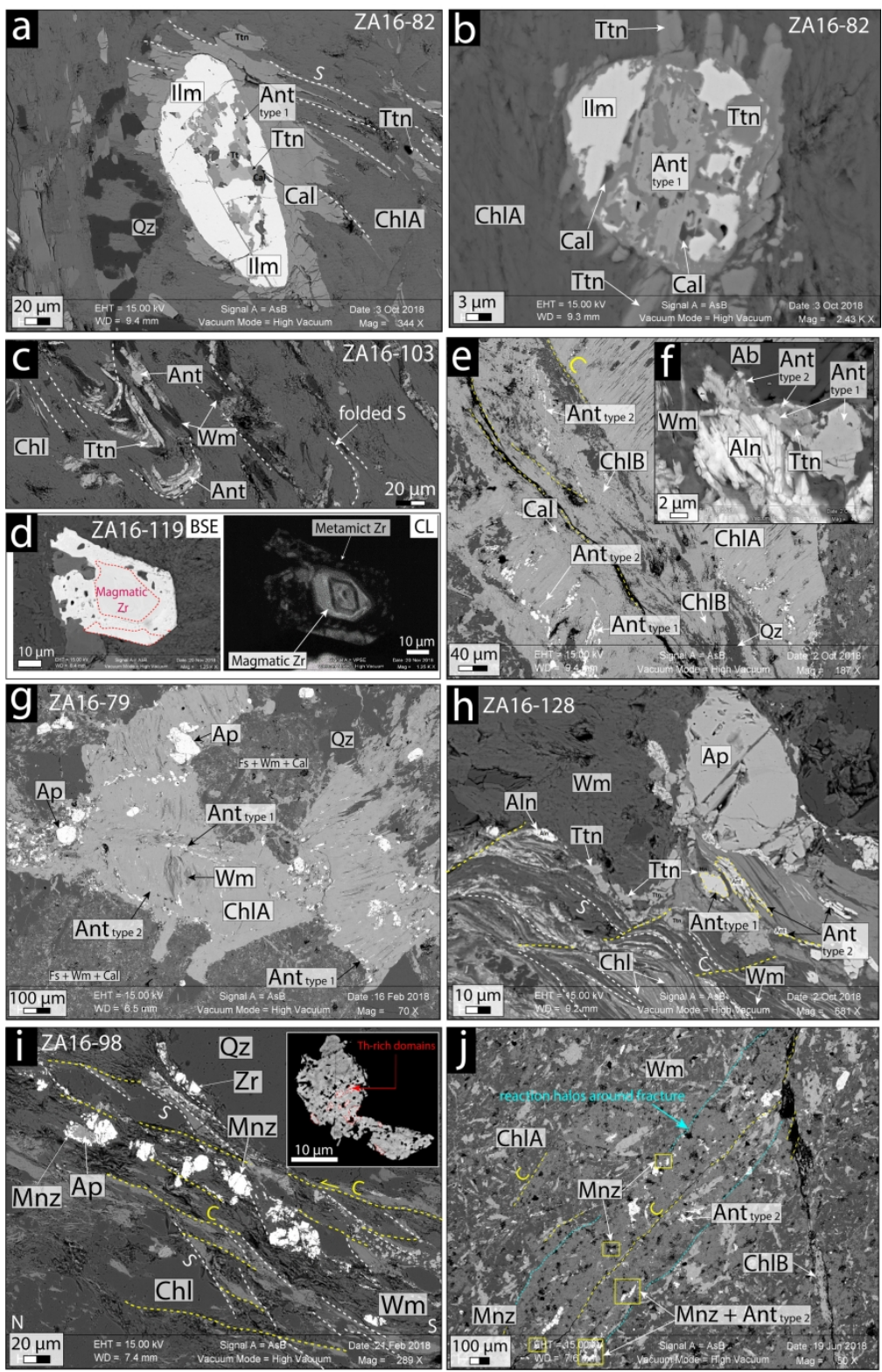

Figure 5. SEM images of accessory minerals in the granodiorite $187 \times 292 \mathrm{~mm}(300 \times 300$ DPI $)$ 


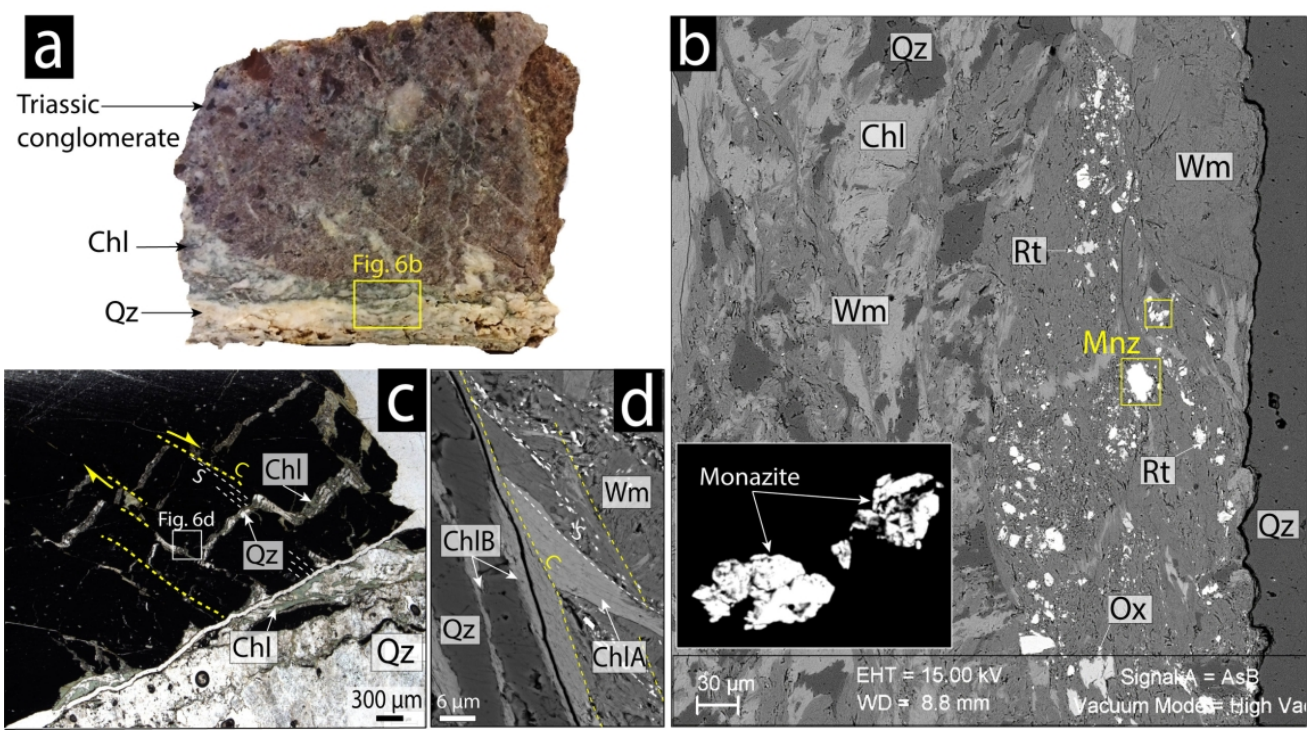

Figure 6. Microviews of Triassic sediments $197 \times 110 \mathrm{~mm}(300 \times 300$ DPI $)$ 

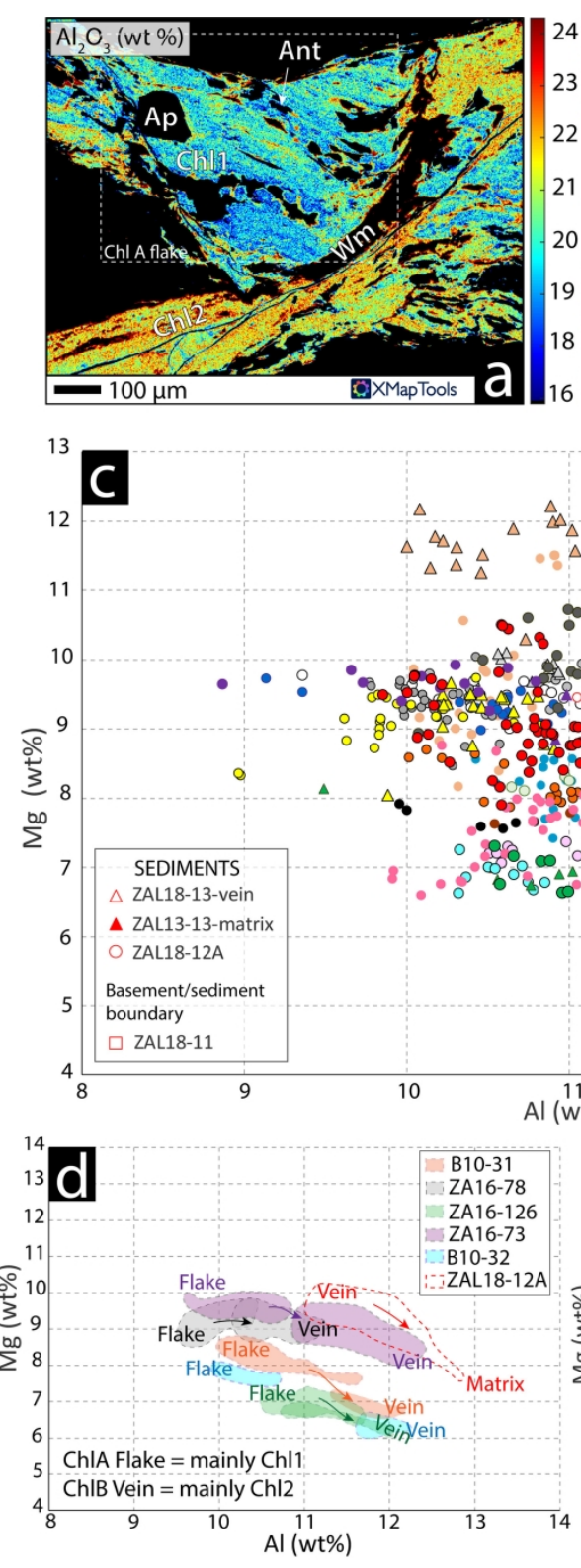

Figure 7. Chlorite chemical composition

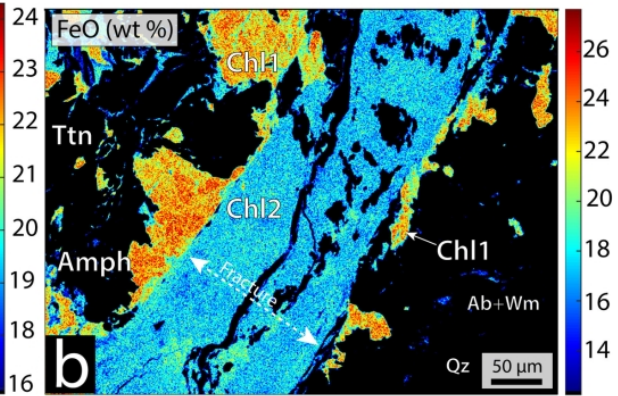

BASEMENT O ZA16-82_flake $\triangle$ ZA16-82_vein OB11-19_flake $\triangle$ B11_19_vein OZA16-98 - B11-31 OZA16-85 - ZA16-97 O za16-79 O ZA16-96 - ZA16-84 O B10-31_flake $\triangle$ B10-31_vein A B10-32_vein - B10-32_flake O ZA16-78_flake $\triangle$ ZA16-78_vein - za16-133 - B11-16 - ZA16-73_flake \ZA16-73_vein OZA16-128_flake - ZA16-117_flake $\triangle$ ZA16-126_flake - ZA16-126_vein - ZA16-93_flake

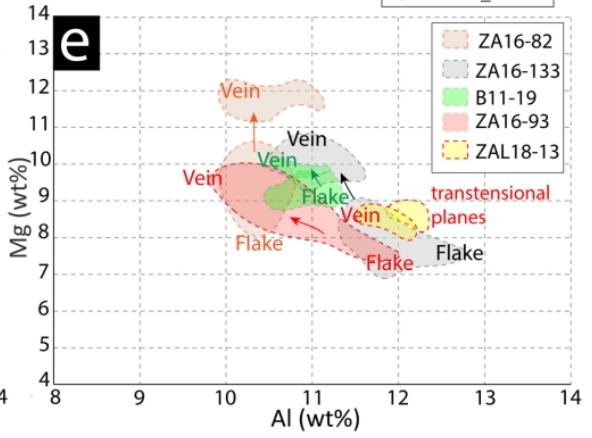

$189 \times 254 \mathrm{~mm}(300 \times 300 \mathrm{DPI})$ 


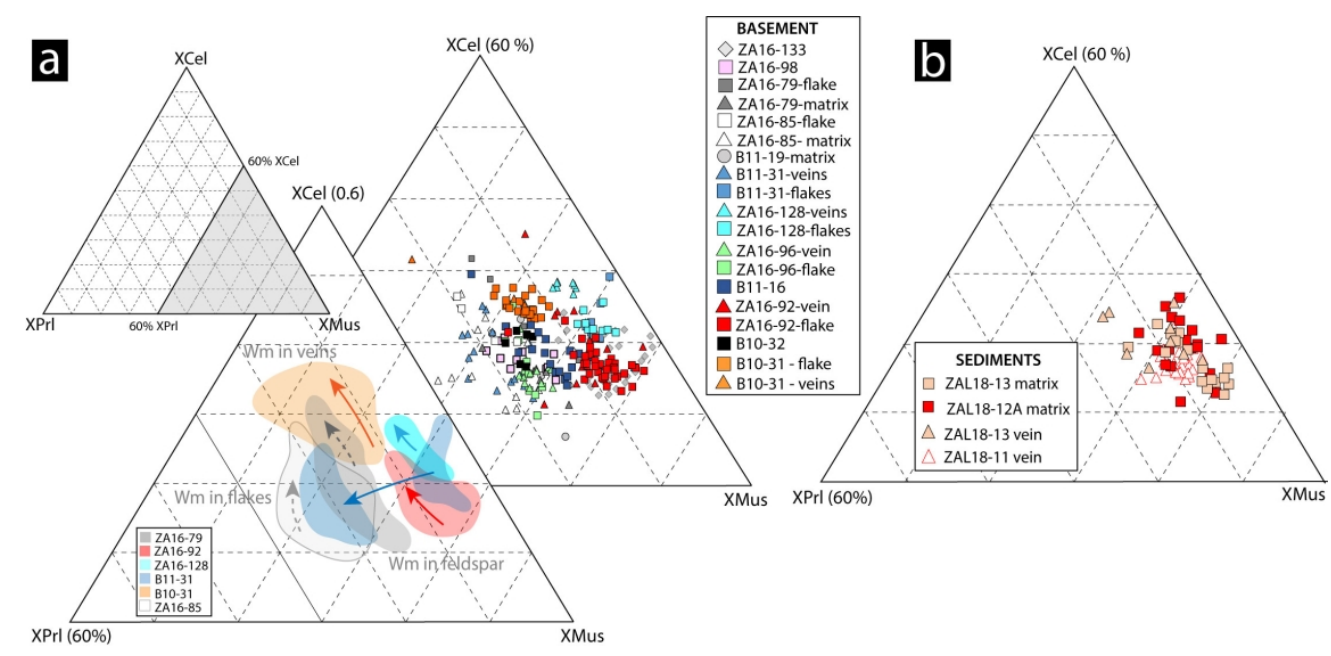

Figure 8. White mica chemical composition

$190 \times 92 \mathrm{~mm}(300 \times 300 \mathrm{DPI})$ 


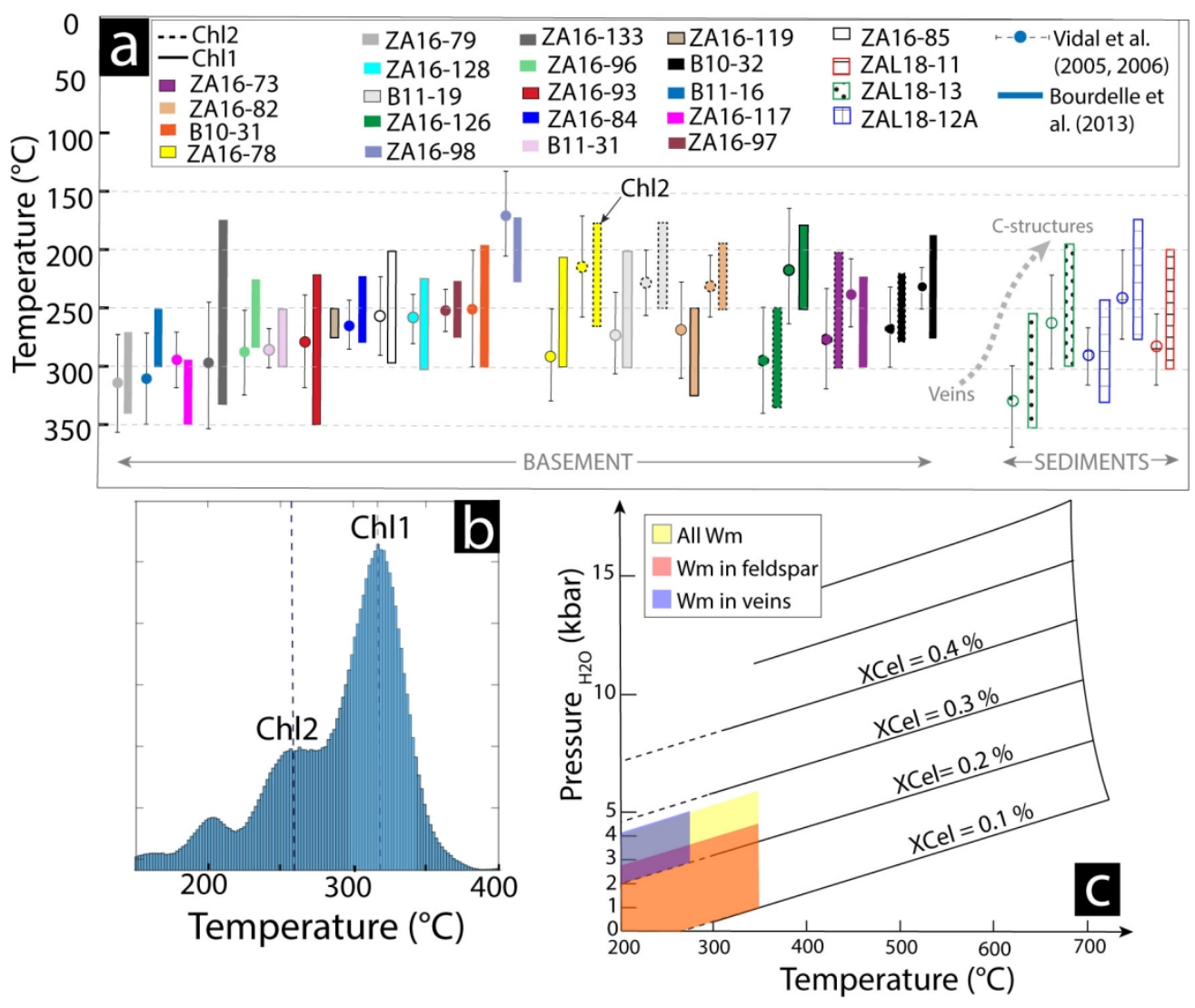

Figure 9. Thermobarometry

$149 \times 125 \mathrm{~mm}(300 \times 300$ DPI $)$ 


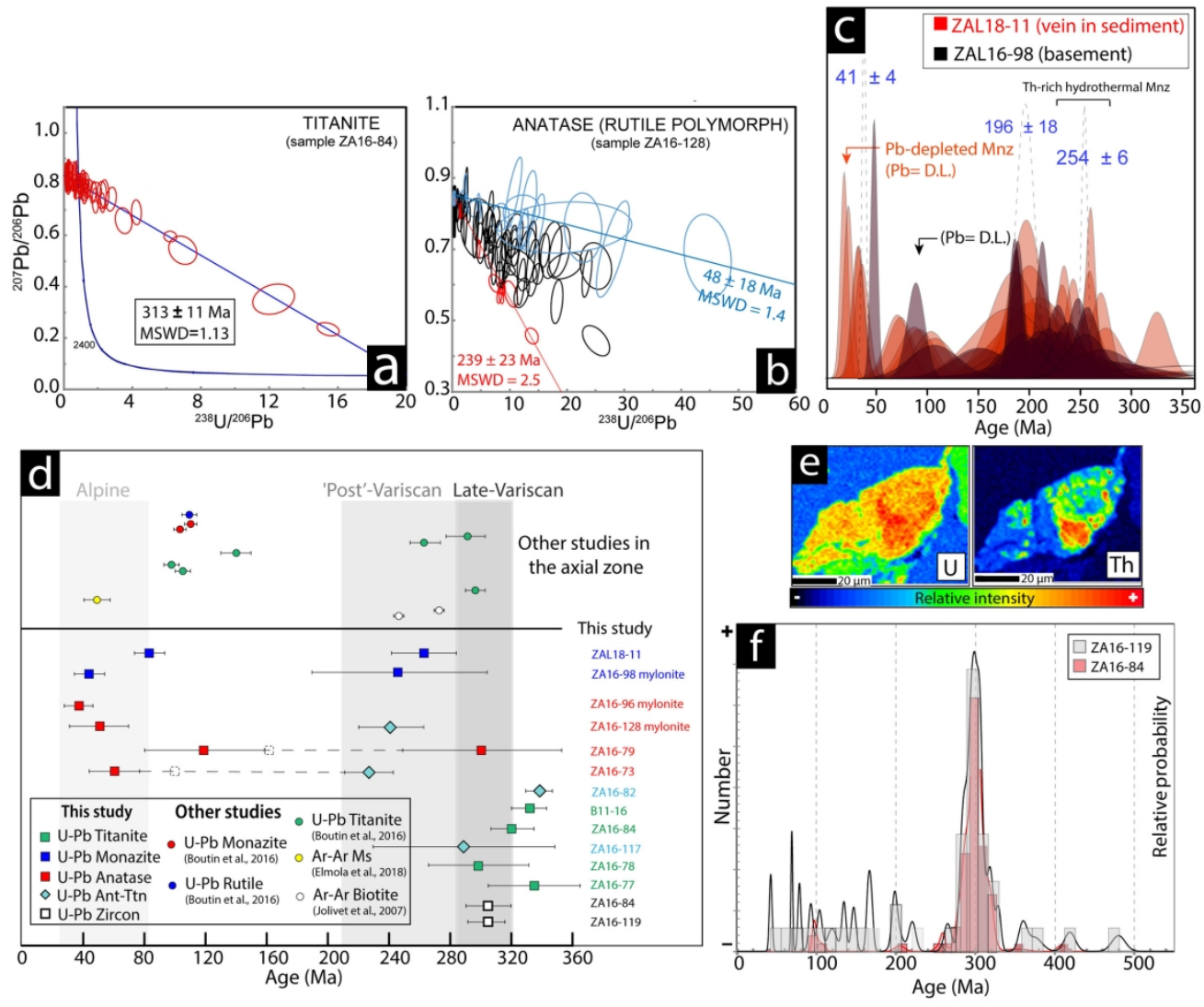

Figure 10. Geochronological results

$189 \times 166 \mathrm{~mm}(300 \times 300$ DPI) 


\begin{tabular}{|c|c|c|c|c|c|c|}
\hline Sample & Deformation & $\begin{array}{c}\text { Ttn age } \\
\text { (Ma) }\end{array}$ & $\begin{array}{c}\text { Ant age } \\
\text { (Ma) }\end{array}$ & $\begin{array}{c}\text { Mnz age } \\
\text { (Ma) }\end{array}$ & $\begin{array}{c}\mathrm{Zr} \text { age } \\
\text { (Ma) }\end{array}$ & Comments \\
\hline B11-16 & undeformed & $336 \pm 12$ & & & & \\
\hline ZA16-119 & undeformed & & & & $\begin{array}{c}310 \pm 20 \\
285-291(?) \\
\end{array}$ & Metamict zircon domains \\
\hline ZA16-117 & undeformed & $\begin{array}{l}337 \pm 20 \\
293 \pm 59\end{array}$ & & & & Relicts of anatase in titanite \\
\hline ZA16-84 & undeformed & $313 \pm 11$ & & & $300 \pm 10$ & \\
\hline ZA16-77 & little deformed & $337 \pm 34$ & & & & \\
\hline ZA16-79 & $\begin{array}{c}\text { distributed to } \\
\text { discretely deformed }\end{array}$ & & $\begin{array}{l}301 \pm 56 \\
120 \pm 39\end{array}$ & & & $\begin{array}{c}\text { Difficult distinction between two } \\
\text { anatase age populations }\end{array}$ \\
\hline ZA16-82 & discretely deformed & $339 \pm 7$ & & & & Relicts of anatase in titanite \\
\hline ZA16-78 & discretely deformed & $303 \pm 36$ & & & & Titanite only \\
\hline ZA16-73 & discretely deformed & & $\begin{array}{c}221 \pm 19 \\
60 \pm 18\end{array}$ & & & \\
\hline ZA16-128 & $\begin{array}{c}\text { discretely deformed to } \\
\text { mylonite }\end{array}$ & & $\begin{array}{c}239 \pm 23 \\
48 \pm 18\end{array}$ & & & \\
\hline ZA16-96 & mylonite & & $39 \pm 8$ & & & Low variability of initial $\mathrm{Pb}$ \\
\hline ZA16-98 & (proto-)mylonite & & & $\begin{array}{c}254 \pm 6 \\
<50\end{array}$ & & $\begin{array}{l}\text { Ages }<100 \mathrm{Ma} \text { for } \mathrm{Pb}<\mathrm{D} . \mathrm{L} . \\
\text { Ages }<50 \mathrm{Ma} \text { for } \mathrm{Mnz}-\mathrm{Thr}\end{array}$ \\
\hline ZA16-11 & Triassic sediment & & & $\begin{array}{l}196 \pm 18 \\
<20\end{array}$ & & Ages $<20 \mathrm{Ma}$ for $\mathrm{Pb}<$ D.L. \\
\hline
\end{tabular}

Table 1 Summary of sample characteristic and ages. 


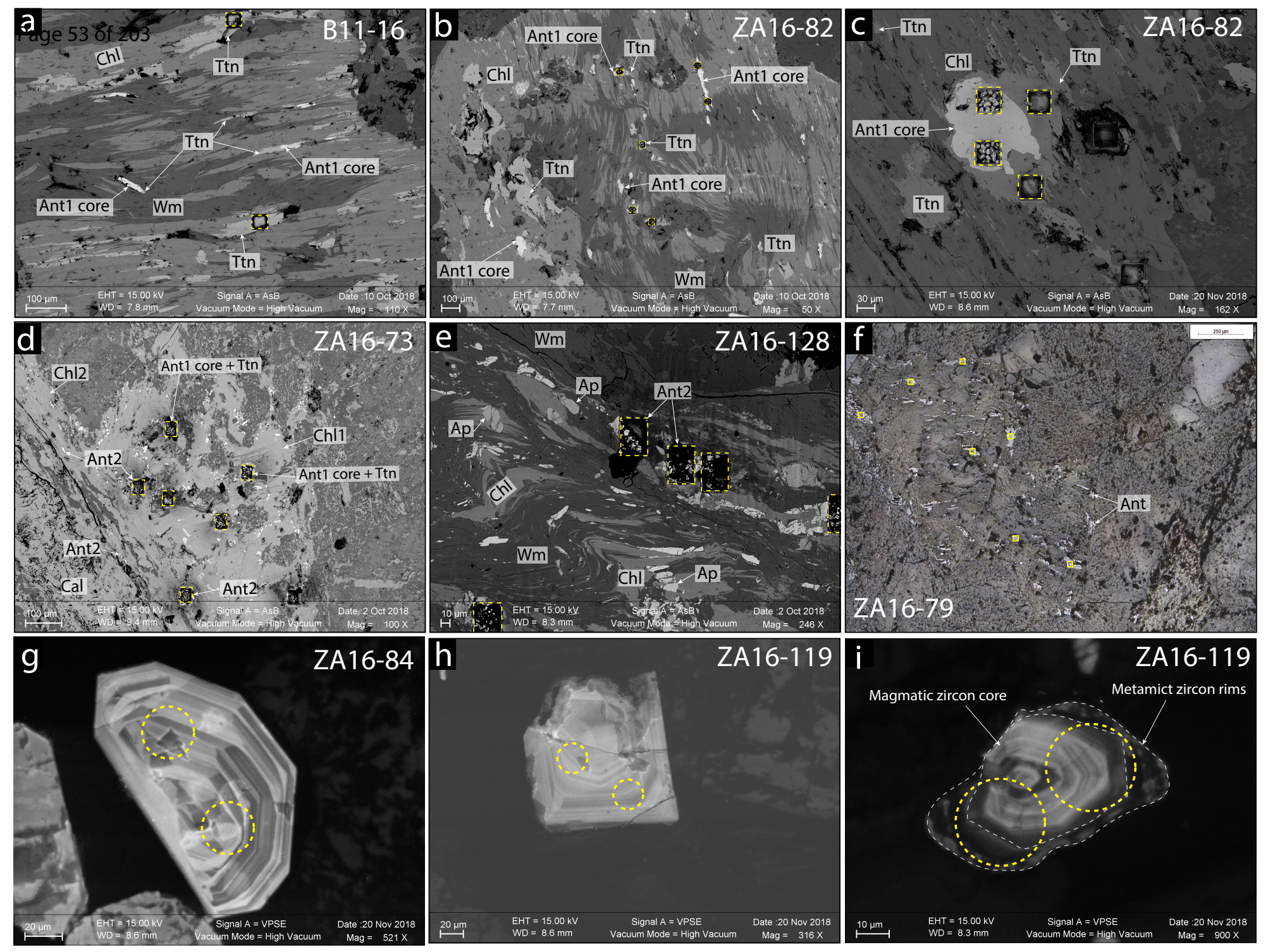

Figure S1. Examples of the location of the laser spots for dating on BSE images (a-e, g-i) and under reflected light (f). Laser spot location is indicated by the yellow dashed square (anatase and titanite) or circle (zircon). 
TITANITE
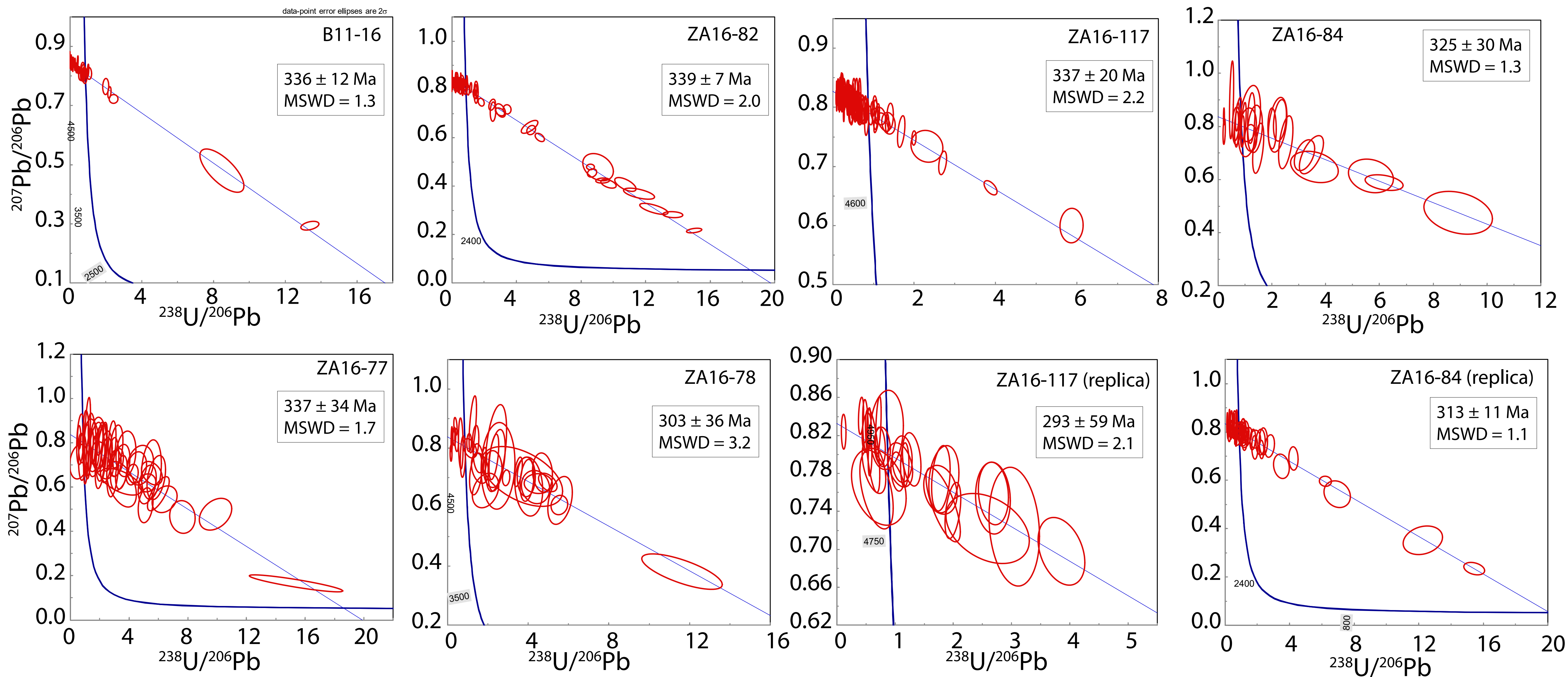

ANATASE
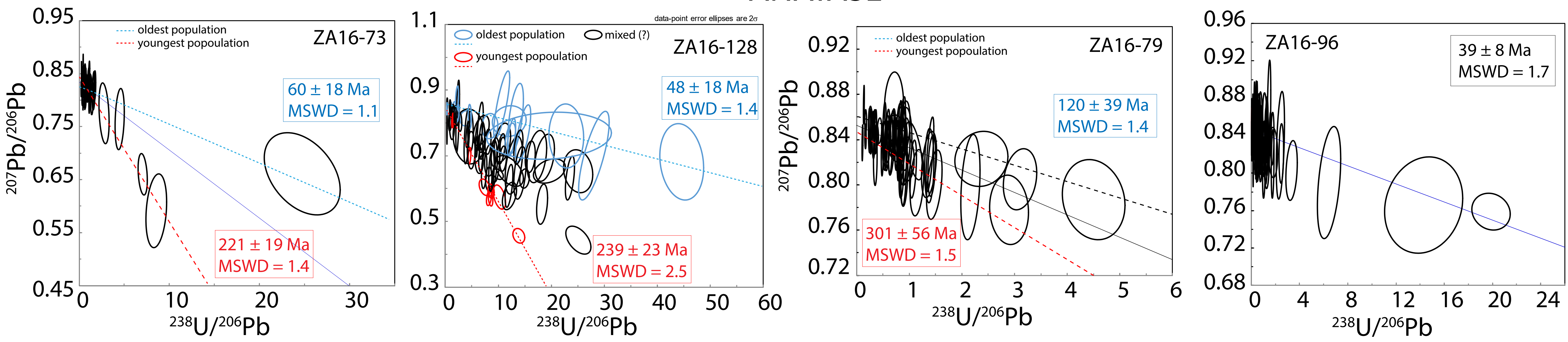

\section{ZIRCON}
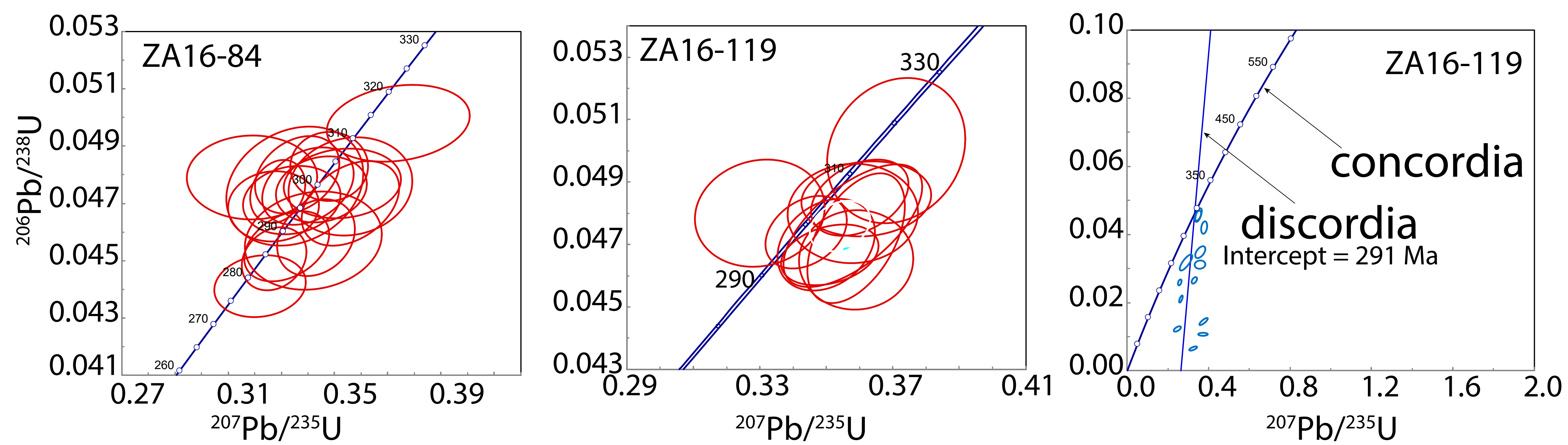

Fig. S1. Tera-Wasserburg diagrams for dated titanite and anatase, concordia diagrams for zircon. 
1 Appendix S1. Analytical methods for geochronology and monazite analysis

2

\section{Protocol used for monazite EPMA compositional analysis}

Monazite was analyzed by electron probe micro-analysis (EPMA) with a JEOL JXA-8230 instrument at the Institute des Sciences de la Terre (ISTerre, Grenoble) on thin sections coated with a 55-60 nm thick layer of carbon to avoid material degradation during analysis at high beam current (Montel et al., 2011). Standards were coated in the same way. For the point analyses, the acceleration voltage was set at $15 \mathrm{keV}$ with a spot beam size and a beam current of $200 \mathrm{nA}$. The duration of each analysis was $\sim 9$ minutes. During this exposure time, the evolution of the absorbed current was negligible $(<5 \mathrm{nA})$.

Quantitative analyses were carried out using the X-ray lines: $\operatorname{SiK} \alpha, \mathrm{UM} \beta, \mathrm{ThM} \alpha, \mathrm{CaK} \alpha, \mathrm{PK} \alpha$, YL $\alpha, \operatorname{DyL} \beta, \operatorname{GdL} \beta, \operatorname{SmL} \beta, \operatorname{PrL} \beta, \operatorname{NdL} \alpha, \operatorname{CeL} \alpha, \operatorname{LaL} \alpha, \operatorname{PbM} \beta$. Background positions were carefully selected with high sensitivity wavelength scans before and after the element signal. The background value for each line was estimated by linear interpolation. A calibrated overlap correction of 0.0079 was applied for peak interference of ThM $\alpha$ on UM $\beta$. A standard monazite (Madagascar, Paquette et al., 2007) was analyzed before and after the unknown analyses of the target ( 5 points each time) to track possible instrumental drift and to verify the consistency of the age calculated for the monazite grains. Other standards used were $\mathrm{ThO}_{2}$ for thorium, $\mathrm{UO}_{2}$ for uranium and crocoite $\left(\mathrm{PbCrO}_{4}\right)$ for lead. ZAF correction procedure was applied using the JEOL software for quantitative analysis. EPMA X-rays maps of monazite grains was done for Si, U, Th, Ca using an acceleration voltage of $15 \mathrm{kV}$, a beam current of $100 \mathrm{nA}$ with a focused beam and a counting time per pixel of $500 \mathrm{~ms}$.

$\mathrm{U}$, Th and $\mathrm{Pb}$ contents were measured with counting time (peak plus background) of 480 s for $\mathrm{Pb}, 300 \mathrm{~s}$ for $\mathrm{U}$ and $160 \mathrm{~s}$ for Th. The detection limit associated were $90 \mathrm{ppm}$ for $\mathrm{Pb}, 120 \mathrm{ppm}$ for $\mathrm{U}$ and $115 \mathrm{ppm}$ for Th. For each monazite analysis, a chemical U-Th-Pb age was determined (Montel et al., 1996). Ages obtained for the standard monazite Madagascar cluster around 552 
$\pm 4 \mathrm{Ma}$. Monazite analyses in studied samples were divided into populations on the base of their composition (e.g. $\mathrm{ThO}_{2}$ content, see the main text for details). The average age of each population was calculated using the software NiLeDAM (Seydoux-Guillaume et al., 2012).

\section{2. $\mathrm{U} / \mathrm{Pb}$ geochronology: titanite, rutile and zircon standards}

Titanite and anatase (rutile polymorph) were dated in-situ by the U-Pb LA-ICP-MS technique at Trinity College, Dublin (Ireland). U-Pb ages were determined using a Photon Machines Analyte Exite 193 nm ArF Excimer laser-ablation system with a Helex 2-volume ablation cell coupled to an Agilent 7900 ICPMS. The ICPMS was tuned using NIST 612 standard glass to yield $\mathrm{Th} / \mathrm{U}$ ratios of unity and low oxide production rates $\left(\mathrm{ThO}^{+} / \mathrm{Th}^{+}\right.$typically $\left.<0.15 \%\right) .0 .15$ 1/min He carrier gas was fed into the cell body and $0.251 /$ min He was fed into the cup. The aerosol was subsequently mixed with 0.6 1/min Ar make-up gas and a small volume of $\mathrm{N}_{2}$ (ca. $10 \mathrm{ml} / \mathrm{min}$ ) in a c. $1 \mathrm{~cm}^{3}$ signal smoothing device to enhance signal sensitivity and reduce oxide formation. NIST612 was used as the trace element primary standard for titanite, rutile and zircon. For titanite analysis, OLT-1 (Kennedy et al., 2010) was used as the primary standard for titanite geochronology (published TIMS ages of $1014.8 \pm 2 \mathrm{Ma}$, obtained age of $1018 \pm 5$ ma) while MKED (Spandler et al., 2016) was used as a secondary age standard (TIMS age of $1517.3 \pm 0.3$, obtained age of $1541 \pm 8 \mathrm{Ma}$ ). The trace elements analysed were: ${ }^{90} \mathrm{Zr},{ }^{139} \mathrm{La},{ }^{51} \mathrm{~V}$, ${ }^{93} \mathrm{Nb},{ }^{43} \mathrm{Ca},{ }^{153} \mathrm{Eu},{ }^{157} \mathrm{Gd},{ }^{202} \mathrm{Hg},{ }^{204} \mathrm{~Pb},{ }^{206} \mathrm{~Pb},{ }^{207} \mathrm{~Pb},{ }^{208} \mathrm{~Pb},{ }^{232} \mathrm{Th},{ }^{238} \mathrm{U},{ }^{172} \mathrm{Yb},{ }^{147} \mathrm{Sm},{ }^{181} \mathrm{Ta}$. For analysis of rutile polymorphs, rutile R10 (Luvizotto et al., 2009) was used as the primary standard (U-Ob TIMS age of 1090 Ma, obtained age of $1089 \pm 5$ Ma). Rutile R19 (TIMS age of $489.5 \pm 0.9 \mathrm{Ma}$, obtained age of $491 \pm 11$ ) and PCA-S207 rutile (age of 1865.0 $\pm 7.5 \mathrm{Ma}$, obtained age of $1921 \pm 36$ ) was used as secondary standards (Zack et al., 2011; Bracciali et al., 2013). The trace elements analyzed were $\mathrm{Al},{ }^{43} \mathrm{Ca},{ }^{49} \mathrm{Ti},{ }^{24} \mathrm{Mg},{ }^{51} \mathrm{~V},{ }^{52} \mathrm{Cr},{ }^{57} \mathrm{Fe},{ }^{89} \mathrm{Y},{ }^{90} \mathrm{Zr},{ }^{93} \mathrm{Nb}$, ${ }^{118} \mathrm{Sn},{ }^{121} \mathrm{Sb},{ }^{181} \mathrm{Ta},{ }^{182} \mathrm{~W},{ }^{202} \mathrm{Hg},{ }^{204} \mathrm{~Pb},{ }^{206} \mathrm{~Pb},{ }^{207} \mathrm{~Pb},{ }^{208} \mathrm{~Pb},{ }^{232} \mathrm{Th}$ and ${ }^{238} \mathrm{U}$. For zircon analysis 
the primary standard employed was zircon 91500. The zircon age standards WRS1348 (TIMS age of $526.26 \pm 0.70$, obtained age of $523.4 \pm 2.1 \mathrm{Ma}$ ), Plesovice and Temora-2 (TIMS age of $416.8 \pm 1.3$, obtained age of $416.5 \pm 2.6 \mathrm{Ma}$ ) were used as secondary age standards (Chew et al, 2019 and references therein). The trace elements analysed were: ${ }^{49} \mathrm{Ti},{ }^{90} \mathrm{Zr},{ }^{202} \mathrm{Hg},{ }^{204} \mathrm{~Pb}$, ${ }^{206} \mathrm{~Pb},{ }^{207} \mathrm{~Pb},{ }^{208} \mathrm{~Pb},{ }^{232} \mathrm{Th}$ and ${ }^{238} \mathrm{U}$. Three of each standard were analysed under the same conditions as the unknowns, with each block of unknowns comprising 20-30 analyses.

The raw titanite and rutile isotope data were reduced using the "VizualAge UcomPbine" data reduction scheme (DRS) of Chew et al. (2014), a modification of the U-Pb geochronology "VizualAge" DRS of Petrus \& Kamber (2012) that can account for the presence of variable common $\mathrm{Pb}$ in the primary standard materials. The "VizualAge" DRS was employed for zircon data reduction. Both DRSs runs within the freeware IOLITE package of Paton et al. (2011). In IOLITE, user-defined time intervals are established for the baseline correction procedure to calculate session-wide baseline-corrected values for each isotope. The time-resolved fractionation response of individual standard analyses is then characterised using a userspecified down-hole correction model (such as an exponential curve, a linear fit or a smoothed cubic spline). The VizualAge data reduction scheme then fits this appropriate session-wide "model" U-Th- $\mathrm{Pb}$ fractionation curve to the time-resolved standard data and the unknowns. Sample-standard bracketing is applied after the correction of down-hole fractionation to account for long-term drift in isotopic or elemental ratios by normalizing all ratios to those of the $\mathrm{U}-\mathrm{Pb}$ reference standards. Common $\mathrm{Pb}$ in the titanite and rutile standards was corrected using the ${ }^{207} \mathrm{~Pb}$-based correction method.

\section{References}


Black, L. P., Kamo, S. L., Allen, C. M., Aleinikoff, J. N., Davis, D. W., Korsch, R. J. \& Foudoulis, C. (2003). TEMORA 1: A new zircon standard for Phanerozoic U-Pb geochronology. Chemical Geology, 200, 155-170.

Bracciali, L., Parrish, R. R., Horstwood, M. S. A., Condon, D. J., \& Najman, Y. (2013). U-Pb LA-(MC)-ICP-MS dating of rutile: New reference materials and applications to sedimentary provenance. Chemical Geology, 347, 82-101.

Chew, D., Drost, K. \& Petrus, J. A. (2019). Ultrafast, > 50 Hz LA-ICP-MS Spot Analysis Applied to U-Pb Dating of Zircon and other U-Bearing Minerals. Geostandards and Geoanalytical Research, 43(1), 39-60.

Chew, D., Petrus, J.A. \& Kamber, B.S. (2014). U-Pb LA-ICPMS dating using accessory mineral standards with variable common $\mathrm{Pb}$. Chemical Geology, 363 185-199

Kennedy, A.K., Kamo, S.L., Nasdala, L., Timms,N.E., 2010. Grenville Skarn Titanite: potential reference material for SIMS U-Th-Pb analysis. Can. Mineral. 48 (6), $1423-$ 1443.

Luvizotto, G.L., Zack, T., Meyer, H.P., Ludwig, T., Triebold, S., Kronz, A., Münker, C., Stockli, D.F., Prowatke, S., Klemme, S., Jacob, D.E., von Eynatten, H., 2009. Rutile crystals as potential trace element and isotope mineral standards for microanalysis. Chem. Geol. 261 (3-4), 346-369.

Montel, J.-M., Foret, S., Veschambre, M., Nicollet, C. \& Provost, A. (1996). Electron microprobe dating of monazite. Chemical Geology, 131, 37-53. 
Montel, J.M., Razafimahatratra, D., Ralison, B., De Parseval, P., Thibault, M. \& Randranja, R. (2011). Monazite from mountain to ocean: a case study from Trolognaro (Fort-Dauphin), Madagascar. European Journal of Mineralogy 23(5), 745-757.

Paquette, J. L. \& tiepolo, M. (2007). High resolution $(5 \mu \mathrm{m})$ U-Th-Pb isotope dating of monazite with excimer laser ablation (ELA)-ICPMS, Chemical Geology, 240(3-4), $222-237$

Pointon, M. A., Cliff, R. A. \& Chew, D. M. (2012). The provenance of Western Irish Namurian Basin sedimentary strata inferred using detrital zircon U-Pb LA-ICP-MS geochronology. Geological Jounral, 47, 77-98.

Seydoux-Guillaume, A.-M., Montel, J.-M., Bingen, B., Bosse, V., de Parseval, P., Paquette, J.L., Janots, E. \& Wirth, R. (2012). Low-temperature alteration of monazite : Fluid mediated coupled dissolution-preciputation, irradiation damage and disturbance of the U-Pb geochronology. Chemical Geology, 330-331, 140-158.

Spandler, C., Hammerli, J., Sha, P., Hilbert-Wolf, H., Hu, Y., Roberts, E. \& Schmitz, M. (2016). MKED1: A new titanite standard for in situ analysis of $\mathrm{Sm}-\mathrm{Nd}$ isotopes and $\mathrm{U}-\mathrm{Pb}$ geochronology. Chemical Geology, 425, 110-126.

Zack, T., Stockli, D. F., Luvizotto, G. L., Barth, M. G., Belousova, E., Wolfe, M. R. \& Hinton, R. W. (2011). In situ U-Pb rutile dating by LA-ICP-MS: Pb-208 correction and prospects for geological applications. Contributions to Mineralogy and Petrology, 162, 515-530. 


\begin{tabular}{|c|c|c|c|c|c|c|c|c|c|c|c|c|c|c|c|}
\hline Sample & \multicolumn{2}{|c|}{ ZA16-82 } & \multicolumn{3}{|c|}{ B11-19 } & \multicolumn{3}{|c|}{ ZA16-98 } & \multicolumn{3}{|c|}{ B11-31 } & \multicolumn{3}{|c|}{ ZA16-85 } & ZA16-97 \\
\hline $\begin{array}{c}\text { Lat } / \text { Long } \\
\text { Deformation }\end{array}$ & \multicolumn{2}{|c|}{$\begin{array}{c}42^{\circ} 40^{\prime} 7.3^{\prime \prime} \mathrm{N} / 0^{\circ} 16^{\prime} 43.3^{\prime \prime} \mathrm{E} \\
\text { Discretely deformed }\end{array}$} & \multicolumn{3}{|c|}{$\begin{array}{c}42^{\circ} 40^{\prime} 6.9^{\prime \prime} \mathrm{N} / 0^{\circ} 16^{\prime} 42.9^{\prime \prime} \mathrm{E} \\
\text { Discretely deformed }\end{array}$} & \multicolumn{3}{|c|}{$\begin{array}{c}42^{\circ} 40^{\prime} 2.3^{\prime \prime} \mathrm{N} / 0^{\circ} 16^{\prime} 31.3^{\prime \prime} \mathrm{E} \\
\text { Mylonite }\end{array}$} & \multicolumn{3}{|c|}{$\begin{array}{c}42^{\circ} 39^{\prime} 51.7^{\prime \prime} \mathrm{N} / 0^{\circ} 16^{\prime} 9.3^{\prime \prime E} \\
\text { Mylonite }\end{array}$} & \multicolumn{3}{|c|}{$\begin{array}{l}42^{\circ} 40^{\prime} 6.1^{\prime \prime} \mathrm{N} / 0^{\circ} 16^{\prime} 40.6^{\prime \prime} \mathrm{E} \\
\text { Distributed deformation }\end{array}$} & \multirow{2}{*}{\begin{tabular}{|c}
$\begin{array}{c}42^{\circ} 40^{\prime} 0.9^{\prime \prime} \mathrm{N} / 0^{\circ} 16^{\prime} 32.4^{\prime \prime} \mathrm{E} \\
\text { Mylonite }\end{array}$ \\
Chl \\
\end{tabular}} \\
\hline Mineral & Chl1 & $\mathrm{Ch} 12$ & Chl1 & $\mathrm{Chl} 2$ & $\mathrm{Wm} 1$ & $\mathrm{Chl}$ & $\mathrm{Wm} 2$ & $\mathrm{Wm} 1$ & $\mathrm{Chl}$ & $\mathrm{Wm} 2$ & Wm1 & Chl & $\mathrm{Wm} 2$ & Wm1 & \\
\hline \multicolumn{16}{|l|}{ Oxides } \\
\hline $\mathrm{SiO} 2$ & 27,60 & 28,37 & 26,80 & 27,74 & 47,62 & 25,65 & 48,08 & 47,50 & 28,28 & 48,70 & 47,43 & 27,41 & 48,66 & 47,39 & 27,09 \\
\hline $\mathrm{TiO} 2$ & 0,07 & 0,05 & 0,09 & 0,02 & 0,02 & 0,11 & 0,17 & 0,02 & 0,17 & 0,16 & 0,15 & 0,12 & 0,06 & 0,20 & 0,02 \\
\hline $\mathrm{A} 12 \mathrm{O} 3$ & 19,81 & 20,57 & 19,82 & 20,76 & 35,97 & 20,07 & 32,35 & 32,42 & 21,25 & 33,60 & 32,27 & 18,96 & 34,52 & 32,82 & 20,80 \\
\hline $\mathrm{FeO}$ & 23,85 & 17,85 & 24,46 & 22,58 & 1,01 & 29,04 & 2,27 & 0,93 & 22,08 & 1,39 & 2,60 & 24,49 & 1,30 & 2,46 & 27,96 \\
\hline $\mathrm{MnO}$ & 0,52 & 0,43 & 0,32 & 0,30 & 0,04 & 0,45 & 0,06 & 0,01 & 0,33 & 0,05 & $<$ D.L. & 0,51 & $<$ D.L. & 0,04 & 0,34 \\
\hline $\mathrm{MgO}$ & 16,28 & 20,26 & 15,27 & 15,92 & 0,78 & 12,10 & 1,58 & 1,11 & 13,70 & 1,28 & 1,40 & 15,98 & 1,15 & 1,92 & 12,90 \\
\hline $\mathrm{CaO}$ & 0,09 & 0,10 & 0,09 & 0,11 & 0,06 & 0,07 & 0,09 & 0,07 & 0,13 & 0,04 & 0,05 & 0,05 & 0,08 & 0,08 & 0,04 \\
\hline $\mathrm{Na} 2 \mathrm{O}$ & 0,07 & 0,02 & 0,05 & $<$ D.L. & 0,19 & 0,01 & 0,16 & 0,32 & 0,04 & 0,16 & 0,24 & 0,02 & 0,41 & 0,42 & 0,03 \\
\hline $\mathrm{K} 2 \mathrm{O}$ & 0,08 & 0,02 & 0,03 & 0,01 & 9,31 & 0,06 & 8,75 & 9,30 & 1,03 & 9,17 & 10,32 & 0,03 & 8,35 & 8,96 & 0,02 \\
\hline Total & 88,38 & 87,67 & 86,93 & 87,44 & 95,02 & 87,56 & 93,52 & 91,68 & 87,03 & 94,55 & 94,47 & 87,64 & 94,52 & 94,30 & 89,21 \\
\hline \multicolumn{16}{|l|}{ Cations } \\
\hline $\mathrm{Si}$ & 2,81 & 2,82 & 2,83 & 2,87 & 3,13 & 2,72 & 3,23 & 3,24 & 2,89 & 3,22 & 3,20 & 2,83 & 3,20 & 3,17 & 2,79 \\
\hline $\mathrm{Ti}$ & 0,01 & 0,03 & 0,01 & 0,00 & $<$ D.L. & 0,01 & 0,01 & 0,00 & 0,01 & 0,01 & 0,01 & 0,01 & 0,00 & 0,01 & 0,00 \\
\hline $\mathrm{Al}$ & 2,38 & 2,33 & 2,47 & 2,53 & 2,79 & 2,51 & 2,56 & 2,61 & 2,56 & 2,62 & 2,56 & 2,31 & 2,68 & 2,59 & 2,52 \\
\hline FeTOT & 1,97 & 1,52 & 2,16 & 1,96 & 0,06 & 2,51 & 0,13 & 0,05 & 1,81 & 0,08 & 0,15 & 2,05 & 0,07 & 0,14 & 2,35 \\
\hline $\mathrm{Mn}$ & 0,05 & 0,03 & 0,03 & 0,03 & 0,00 & 0,04 & 0,00 & 0,00 & 0,03 & 0,00 & 0,00 & 0,04 & 0,00 & 0,00 & 0,03 \\
\hline $\mathrm{Mg}$ & 2,47 & 2,85 & 2,41 & 2,46 & 0,08 & 1,91 & 0,16 & 0,11 & 2,09 & 0,13 & 0,14 & 2,46 & 0,11 & 0,19 & 1,98 \\
\hline $\mathrm{Ca}$ & 0,01 & 0,04 & 0,01 & 0,01 & 0,00 & 0,01 & 0,01 & 0,00 & 0,01 & 0,00 & 0,00 & 0,01 & 0,01 & 0,01 & 0,00 \\
\hline $\mathrm{Na}$ & 0,01 & 0,01 & 0,01 & 0,00 & 0,02 & 0,00 & 0,02 & 0,04 & 0,01 & 0,02 & 0,03 & 0,00 & 0,05 & 0,06 & 0,01 \\
\hline $\mathrm{K}$ & 0,01 & 0,00 & 0,00 & 0,00 & 0,78 & 0,01 & 0,75 & 0,81 & 0,13 & 0,77 & 0,89 & 0,00 & 0,70 & 0,77 & 0,00 \\
\hline Oxygens & 14 & 14 & 14 & 14 & 11 & 14 & 11 & 11 & 14 & 11 & 11 & 14 & 11 & 11 & 14 \\
\hline$X M g$ & 0,63 & 0,78 & 0,62 & 0,65 & 0,58 & 0,50 & 0,55 & 0,68 & 0,65 & 0,62 & 0,49 & 0,62 & 0,61 & 0,58 & 0,52 \\
\hline AlVI & 1,20 & 1,18 & 1,31 & 1,41 & - & 1,24 & - & - & 1,46 & - & - & 1,15 & - & - & 1,31 \\
\hline Octahedral sum & 5,69 & 5,58 & 5,81 & 5,76 & - & 5,71 & - & - & 5,39 & - & - & 5,71 & - & - & 5,67 \\
\hline Xceladonite & - & - & - & - & 0,07 & - & 0,17 & 0,14 & - & 0,14 & 0,21 & - & 0,10 & 0,19 & - \\
\hline Xmuscovite & - & - & - & - & 0,74 & - & 0,62 & 0,68 & - & 0,67 & 0,69 & - & 0,64 & 0,62 & - \\
\hline Xpyrophyllite & - & - & - & - & 0,17 & - & 0,18 & 0,14 & - & 0,18 & 0,07 & - & 0,21 & 0,14 & - \\
\hline
\end{tabular}


Page 61 of 203

Wm1: in flakes or feldspar

Wm2: in the matrix/shear planes

Discretely deformed $=$ fractures

\begin{tabular}{|c|c|c|c|c|c|c|c|c|c|c|c|c|c|c|c|}
\hline Sample & ZA16-84 & \multicolumn{3}{|c|}{ ZA16-96 } & \multicolumn{3}{|c|}{ ZA16-79 } & \multicolumn{3}{|c|}{ B10-31 } & \multicolumn{3}{|c|}{ B10-32 } & \multicolumn{2}{|c|}{ ZA16-78 } \\
\hline $\begin{array}{c}\text { Lat/Long } \\
\text { Deformation }\end{array}$ & $\begin{array}{c}42^{\circ} 40^{\prime} 7.3^{\prime \prime} \mathrm{N} / 0^{\circ} 16^{\prime} 43.3^{\prime \prime} \mathrm{E} \\
\text { Undeformed }\end{array}$ & \multicolumn{3}{|c|}{$\begin{array}{c}42^{\circ} 39^{\prime} 59.1 " \mathrm{~N} / 0^{\circ} 16^{\prime} 35.1 " \mathrm{E} \\
\text { Mylonite }\end{array}$} & \multicolumn{3}{|c|}{$\begin{array}{c}42^{\circ} 39 ' 58.9^{\prime \prime} \mathrm{N} / 0^{\circ} 17^{\prime} 8.95 " \mathrm{E} \\
\text { Distributed deformation }\end{array}$} & \multicolumn{3}{|c|}{$\begin{array}{c}42^{\circ} 39^{\prime} 49.2^{\prime \prime} \mathrm{N} / 0^{\circ} 17^{\prime} 4.4^{\prime \prime} \mathrm{E} \\
\text { Mylonite }\end{array}$} & \multicolumn{3}{|c|}{$\begin{array}{c}42^{\circ} 39^{\prime} 46.3^{\prime \prime} \mathrm{N} / 0^{\circ} 17^{\prime} 2.0^{\prime \prime} \mathrm{E} \\
\text { Mylonite }\end{array}$} & \multicolumn{2}{|c|}{$\begin{array}{c}42^{\circ} 39^{\prime} 59.4^{\prime \prime} \mathrm{N} / 0^{\circ} 17^{\prime} 8.5^{\prime \prime} \mathrm{E} \\
\text { Discretely deformed }\end{array}$} \\
\hline Mineral & $\mathrm{Chl}$ & $\mathrm{Chl}$ & Wm1 & $\mathrm{Wm} 2$ & Chl1 & Wm1 & $\mathrm{Wm} 2$ & Chll & $\mathrm{Chl} 2$ & $\mathrm{Wm}$ & Chl1 & $\mathrm{Ch} 12$ & $\mathrm{Wm}$ & Chl1 & Chl2 \\
\hline \multicolumn{16}{|l|}{ Oxides } \\
\hline $\mathrm{SiO} 2$ & 29,59 & 27,14 & 48,35 & 49,23 & 26,72 & 44,97 & 47,78 & 27,37 & 26,78 & 49,30 & 27,64 & 26,72 & 49,67 & 28,60 & 27,42 \\
\hline $\mathrm{TiO} 2$ & 1,56 & $<$ D.L. & 0,10 & 0,22 & 0,21 & 0,11 & 0,14 & 0,24 & 0,01 & 0,06 & 0,02 & 0,02 & 0,04 & 0,69 & 0,03 \\
\hline $\mathrm{Al} 2 \mathrm{O} 3$ & 17,68 & 21,02 & 33,96 & 32,92 & 18,81 & 34,68 & 33,46 & 19,12 & 22,67 & 31,82 & 18,81 & 22,51 & 32,45 & 18,58 & 20,61 \\
\hline $\mathrm{FeO}$ & 22,98 & 24,78 & 1,78 & 1,04 & 23,93 & 2,79 & 1,34 & 26,67 & 28,07 & 3,43 & 29,36 & 29,05 & 2,19 & 23,99 & 24,34 \\
\hline $\mathrm{MnO}$ & 0,32 & 0,90 & 0,03 & 0,04 & 0,31 & 0,04 & 0,00 & 0,42 & 0,53 & 0,02 & 0,21 & 0,18 & $<$ D.L. & 0,35 & 0,51 \\
\hline $\mathrm{MgO}$ & 15,79 & 14,30 & 1,20 & 1,37 & 15,97 & 1,76 & 1,43 & 14,42 & 10,86 & 2,64 & 13,12 & 10,36 & 1,63 & 14,93 & 14,44 \\
\hline $\mathrm{CaO}$ & 0,53 & 0,04 & 0,03 & $<$ D.L. & 0,04 & 0,07 & 0,10 & 0,03 & 0,05 & 0,03 & 0,03 & 0,05 & 0,07 & 0,15 & 0,11 \\
\hline $\mathrm{Na} 2 \mathrm{O}$ & $<$ D.L. & 0,02 & 0,09 & 0,11 & 0,04 & 0,68 & 0,26 & $<$ D.L. & 0,02 & 0,05 & 0,01 & 0,02 & 0,15 & 0,05 & 0,04 \\
\hline $\mathrm{K} 2 \mathrm{O}$ & 0,33 & 0,03 & 9,25 & 9,35 & 0,02 & 9,37 & 9,16 & 0,02 & 0,10 & 9,66 & 0,06 & 0,03 & 9,85 & 1,12 & 0,06 \\
\hline Total & 88,86 & 88,23 & 94,78 & 94,32 & 86,09 & 94,48 & 93,67 & 88,30 & 89,10 & 97,01 & 89,28 & 88,92 & 96,04 & 88,46 & 87,55 \\
\hline \multicolumn{16}{|l|}{ Cations } \\
\hline $\mathrm{Si}$ & 2,98 & 2,80 & 3,20 & 3,26 & 2,81 & 3,03 & 3,20 & 2,83 & 2,77 & 3,23 & 2,88 & 2,75 & 3,26 & 2,91 & 2,83 \\
\hline $\mathrm{Ti}$ & 0,12 & 0,00 & 0,00 & 0,01 & 0,02 & 0,01 & 0,01 & 0,02 & 0,00 & 0,00 & 0,00 & 0,00 & 0,00 & 0,05 & 0,00 \\
\hline $\mathrm{Al}$ & 2,10 & 2,55 & 2,65 & 2,57 & 2,33 & 2,76 & 2,64 & 2,33 & 2,77 & 2,46 & 2,31 & 2,73 & 2,51 & 2,23 & 2,42 \\
\hline FeTOT & 1,87 & 2,09 & 0,10 & 0,06 & 2,04 & 0,16 & 0,08 & 2,24 & 2,40 & 0,19 & 2,52 & 2,41 & 0,12 & 1,96 & 1,92 \\
\hline $\mathrm{Mn}$ & 0,03 & 0,08 & 0,00 & 0,00 & 0,03 & 0,00 & 0,00 & 0,04 & 0,05 & 0,00 & 0,02 & 0,02 & 0,00 & 0,03 & 0,04 \\
\hline $\mathrm{Mg}$ & 2,37 & 2,20 & 0,12 & 0,14 & 2,50 & 0,18 & 0,14 & 2,22 & 1,68 & 0,26 & 2,04 & 1,59 & 0,16 & 2,27 & 2,39 \\
\hline $\mathrm{Ca}$ & 0,06 & 0,00 & 0,00 & 0,00 & 0,00 & 0,00 & 0,01 & 0,00 & 0,01 & 0,00 & 0,00 & 0,01 & 0,00 & 0,02 & 0,01 \\
\hline $\mathrm{Na}$ & 0,00 & 0,00 & 0,01 & 0,01 & 0,01 & 0,09 & 0,03 & $-0,01$ & 0,00 & 0,01 & 0,00 & 0,00 & 0,02 & 0,01 & 0,00 \\
\hline $\mathrm{K}$ & 0,04 & 0,00 & 0,78 & 0,79 & 0,00 & 0,81 & 0,78 & 0,00 & 0,01 & 0,81 & 0,01 & 0,00 & 0,82 & 0,15 & 0,00 \\
\hline Oxygens & 14 & 14 & 11 & 11 & 14 & 11 & 11 & 14 & 14 & 11 & 14 & 14 & 11 & 14 & 14 \\
\hline$X M g$ & 0,65 & 0,56 & 0,55 & 0,70 & 0,63 & 0,53 & 0,66 & 0,58 & 0,45 & 0,58 & 0,48 & 0,49 & 0,57 & 0,65 & 0,67 \\
\hline AlVI & 1,20 & 1,35 & - & - & 1,15 & - & - & 1,18 & 1,54 & - & 1,20 & 1,47 & - & 1,20 & 1,24 \\
\hline Octahedral sum & 5,47 & 5,72 & - & - & 5,72 & - & - & 5,67 & 5,66 & - & 5,78 & 5,49 & - & 5,46 & 5,59 \\
\hline Xceladonite & - & - & 0,13 & 0,15 & - & 0,16 & 0,14 & - & - & 0,25 & - & - & 0,20 & - & - \\
\hline Xmuscovite & - & - & 0,68 & 0,66 & - & 0,69 & 0,67 & - & - & 0,60 & - & - & 0,64 & - & - \\
\hline Xpyrophyllite & - & - & 0,18 & 0,18 & - & 0,08 & 0,16 & - & - & 0,14 & - & - & 0,14 & - & - \\
\hline
\end{tabular}


Page 62 of 203

\begin{tabular}{|c|c|c|c|c|c|c|c|c|c|c|c|c|c|}
\hline Sample & \multicolumn{3}{|c|}{ ZA16-133 } & \multicolumn{2}{|c|}{ B11-16 } & \multicolumn{2}{|c|}{ ZA16-73 } & \multicolumn{3}{|c|}{ ZA16-128 } & ZA16-117 & \multicolumn{2}{|c|}{ ZA16-126 } \\
\hline $\begin{array}{c}\text { Lat } / \text { Long } \\
\text { Deformation } \\
\end{array}$ & \multicolumn{3}{|c|}{$\begin{array}{c}42^{\circ} 39^{\prime} 49.5^{\prime \prime} \mathrm{N} / 0^{\circ} 16^{\prime} 17.8^{\prime \prime} \mathrm{E} \\
\text { Mylonite }\end{array}$} & \multicolumn{2}{|c|}{$\begin{array}{c}42^{\circ} 40^{\prime} 7.9^{\prime \prime} \mathrm{N} / 0^{\circ} 16^{\prime} 47.9^{\prime \prime} \mathrm{E} \\
\text { Undeformed }\end{array}$} & \multicolumn{2}{|c|}{$\begin{array}{c}42^{\circ} 39^{\prime} 56.2^{\prime \prime} \mathrm{N} / 0^{\circ} 17^{\prime} 6.9^{\prime \prime} \mathrm{E} \\
\text { Discretely deformed }\end{array}$} & \multicolumn{3}{|c|}{$\begin{array}{c}42^{\circ} 39^{\prime} 49.2^{\prime \prime} \mathrm{N} / 0^{\circ} 16^{\prime} 16.6^{\prime \prime} \mathrm{E} \\
\text { Mylonite }\end{array}$} & \multirow{2}{*}{\begin{tabular}{|c|}
$\begin{array}{c}42^{\circ} 39^{\prime} 45.0^{\prime \prime} \mathrm{N} / 0^{\circ} 16^{\prime} 5.7^{\prime \prime} \mathrm{E} \\
\text { Undeformed }\end{array}$ \\
$\mathrm{Chl}$ \\
\end{tabular}} & \multicolumn{2}{|c|}{$\begin{array}{c}42^{\circ} 39^{\prime} 48.9^{\prime \prime} \mathrm{N} / 0^{\circ} 16^{\prime} 17.5^{\prime \prime} \mathrm{F} \\
\text { Mylonite }\end{array}$} \\
\hline Mineral & Chl1 & Chl2 & $\mathrm{Wm}$ & Chl & $\mathrm{Wm}$ & Chl1 & $\mathrm{Chl} 2$ & $\mathrm{Chl}$ & $\mathrm{Wm} 1$ & $\mathrm{Wm} 2$ & & Chl1 & $\mathrm{Ch} 12$ \\
\hline \multicolumn{14}{|l|}{ Oxides } \\
\hline $\mathrm{SiO} 2$ & 26,33 & 27,03 & 48,67 & 26,45 & 49,43 & 28,33 & 26,92 & 26,51 & 48,97 & 48,58 & 26,33 & 26,02 & 28,67 \\
\hline $\mathrm{TiO} 2$ & 0,05 & 0,19 & 0,35 & 0,06 & 0,20 & 1,47 & 0,01 & 0,29 & 0,12 & 0,20 & 0,01 & $<$ D.L & 0,04 \\
\hline $\mathrm{Al} 2 \mathrm{O} 3$ & 23,11 & 21,38 & 34,85 & 21,56 & 34,30 & 18,38 & 22,10 & 19,84 & 32,57 & 31,07 & 19,80 & 21,20 & 23,86 \\
\hline $\mathrm{FeO}$ & 26,22 & 22,15 & 2,41 & 23,85 & 0,84 & 23,74 & 22,90 & 29,63 & 2,04 & 2,93 & 30,71 & 29,50 & 25,93 \\
\hline $\mathrm{MnO}$ & 0,15 & 0,12 & $<$ D.L. & 0,38 & 0,03 & 0,24 & 0,24 & 0,43 & 0,05 & 0,02 & 0,70 & 0,42 & 0,31 \\
\hline $\mathrm{MgO}$ & 13,17 & 17,05 & 1,07 & 15,59 & 1,13 & 16,03 & 15,58 & 11,60 & 1,61 & 2,04 & 11,87 & 11,88 & 8,86 \\
\hline $\mathrm{CaO}$ & 0,04 & 0,10 & 0,03 & 0,08 & 0,07 & 0,18 & 0,09 & 0,06 & 0,08 & 0,11 & 0,06 & 0,07 & 0,11 \\
\hline $\mathrm{Na} 2 \mathrm{O}$ & 0,05 & 0,07 & 0,22 & $<$ D.L. & 0,14 & 0,01 & 0,01 & $<$ D.L. & 0,13 & 0,12 & 0,03 & 0,02 & 0,03 \\
\hline $\mathrm{K} 2 \mathrm{O}$ & 0,02 & 0,04 & 10,55 & $<$ D.L. & 10,07 & 0,23 & $<$ D.L. & 0,11 & 10,73 & 10,75 & 0,04 & $<$ D.L. & 1,80 \\
\hline Total & 89,15 & 88,13 & 98,18 & 88,00 & 96,22 & 88,66 & 87,85 & 88,50 & 96,29 & 95,85 & 89,54 & 89,12 & 89,63 \\
\hline \multicolumn{14}{|l|}{ Cations } \\
\hline $\mathrm{Si}$ & 2,68 & 2,58 & 3,15 & 2,75 & 3,22 & 2,92 & 2,78 & 2,83 & 3,23 & 3,24 & 2,80 & 2,75 & 2,95 \\
\hline $\mathrm{Ti}$ & 0,00 & 0,03 & 0,02 & 0,00 & 0,01 & 0,11 & 0,00 & 0,02 & 0,01 & 0,01 & 0,00 & 0,00 & 0,00 \\
\hline $\mathrm{Al}$ & 2,63 & 2,51 & 2,66 & 2,64 & 2,63 & 2,24 & 2,69 & 2,50 & 2,53 & 2,44 & 2,48 & 2,64 & 2,90 \\
\hline FeTOT & 1,93 & 2,06 & 0,13 & 2,07 & 0,05 & 2,05 & 1,98 & 2,65 & 0,11 & 0,16 & 2,73 & 2,61 & 2,23 \\
\hline $\mathrm{Mn}$ & 0,01 & 0,01 & 0,00 & 0,03 & 0,00 & 0,02 & 0,02 & 0,04 & 0,00 & 0,00 & 0,06 & 0,04 & 0,03 \\
\hline $\mathrm{Mg}$ & 2,57 & 2,53 & 0,10 & 2,42 & 0,11 & 2,47 & 2,40 & 1,85 & 0,16 & 0,20 & 1,88 & 1,87 & 1,36 \\
\hline $\mathrm{Ca}$ & 0,01 & 0,01 & 0,00 & 0,01 & 0,01 & 0,02 & 0,01 & 0,01 & 0,01 & 0,01 & 0,01 & 0,01 & 0,01 \\
\hline $\mathrm{Na}$ & 0,00 & 0,00 & 0,03 & 0,00 & 0,02 & 0,00 & 0,00 & 0,00 & 0,02 & 0,01 & 0,01 & 0,00 & 0,01 \\
\hline K & 0,00 & 0,01 & 0,87 & 0,00 & 0,84 & 0,03 & 0,00 & 0,01 & 0,90 & 0,91 & 0,01 & 0,00 & 0,24 \\
\hline Oxygens & 14 & 14 & 11 & 14 & 11 & 14 & 14 & 14 & 11 & 11 & 14 & 14 & 14 \\
\hline$X M g$ & 0,62 & 0,66 & 0,44 & 0,54 & 0,70 & 0,55 & 0,55 & 0,41 & 0,58 & 0,55 & 0,41 & 0,42 & 0,38 \\
\hline AlVI & 1,31 & 1,13 & - & 1,40 & - & 1,27 & 1,47 & 1,35 & - & - & 1,28 & 1,40 & 1,85 \\
\hline Octahedral sum & 5,82 & 5,73 & - & 5,92 & - & 5,80 & 5,87 & 5,88 & - & - & 5,95 & 5,92 & 5,47 \\
\hline Xceladonite & - & - & 0,17 & - & 0,14 & - & - & - & 0,22 & 0,28 & - & - & - \\
\hline Xmuscovite & - & - & 0,72 & - & 0,72 & - & - & - & 0,69 & 0,65 & - & - & - \\
\hline Xpyrophyllite & - & - & 0,09 & - & 0,13 & - & - & - & 0,07 & 0,05 & - & - & - \\
\hline
\end{tabular}




\begin{tabular}{|c|c|c|c|c|c|c|c|c|c|c|c|c|c|c|}
\hline Sample & ZA16-119 & \multicolumn{4}{|c|}{ ZA16-93 } & \multicolumn{2}{|c|}{ ZAL18-11 } & \multicolumn{3}{|c|}{ ZAL18-12 } & \multicolumn{4}{|c|}{ ZAL18-13 } \\
\hline $\begin{array}{c}\text { Lat/Long } \\
\text { Deformation }\end{array}$ & $\begin{array}{c}42^{\circ} 39^{\prime} 46.4^{\prime \prime} \mathrm{N} / 0^{\circ} 16^{\prime} 14.3^{\prime \prime} \mathrm{E} \\
\text { Undeformed }\end{array}$ & \multicolumn{4}{|c|}{$\begin{array}{l}42^{\circ} 40^{\prime} 1.1^{\prime \prime} \mathrm{N} / 0^{\circ} 16^{\prime} 38.4^{\prime \prime} \mathrm{E} \\
\text { Distributed deformation }\end{array}$} & \multicolumn{2}{|c|}{$\begin{array}{c}42^{\circ} 40^{\prime} 4.6^{\prime \prime} \mathrm{N} / 0^{\circ} 16^{\prime} 41.9^{\prime \prime} \mathrm{E} \\
\text { Vein in sediments } \\
\end{array}$} & \multicolumn{3}{|c|}{$\begin{array}{c}42^{\circ} 40^{\prime} 1.3^{\prime \prime} \mathrm{N} / 0^{\circ} 16^{\prime} 43.4^{\prime \prime} \mathrm{E} \\
\text { Triassic sediments } \\
\end{array}$} & \multicolumn{4}{|c|}{$\begin{array}{c}42^{\circ} 40^{\prime} 4.3^{\prime \prime} \mathrm{N} / 0^{\circ} 16^{\prime} 43.1^{\prime \prime} \mathrm{E} \\
\text { Triassic sediments } \\
\end{array}$} \\
\hline Mineral & Chl & Chl1 & Chl2 & $\mathrm{Wm} 1$ & $\mathrm{Wm} 2$ & Chl2 & $\mathrm{Wm}$ & Chl1 & Chl2 & $\mathrm{Wm}$ & Chl1 & $\mathrm{Chl} 2$ & Wm1 & Wm2 \\
\hline \multicolumn{15}{|l|}{ Oxides } \\
\hline $\mathrm{SiO} 2$ & 27,68 & 27,84 & 25,18 & 48,72 & 48,62 & 27,83 & 48,68 & 27,27 & 28,82 & 47,37 & 26,17 & 27,49 & 47,23 & 49,25 \\
\hline $\mathrm{TiO} 2$ & 0,18 & 0,06 & 0,23 & 0,09 & 0,03 & $<$ D.L. & 0,05 & 0,09 & 0,01 & 0,32 & $<$ D.L. & 0,04 & 0,24 & 0,15 \\
\hline $\mathrm{A} 12 \mathrm{O} 3$ & 17,45 & 20,01 & 21,47 & 33,14 & 33,70 & 23,82 & 34,68 & 23,62 & 22,73 & 35,03 & 23,94 & 22,82 & 35,16 & 32,41 \\
\hline $\mathrm{FeO}$ & 27,88 & 22,63 & 28,73 & 1,17 & 1,36 & 21,27 & 2,59 & 23,23 & 20,77 & 1,09 & 25,03 & 22,89 & 1,73 & 2,55 \\
\hline $\mathrm{MnO}$ & 0,39 & 0,35 & 0,21 & $<$ D.L. & $<$ D.L. & 0,30 & $<$ D.L. & 0,30 & 0,21 & 0,04 & 0,27 & 0,19 & $<$ D.L. & 0,00 \\
\hline $\mathrm{MgO}$ & 13,91 & 17,38 & 11,77 & 1,22 & 1,64 & 13,74 & 0,71 & 12,11 & 16,03 & 0,93 & 13,41 & 14,29 & 0,85 & 0,92 \\
\hline $\mathrm{CaO}$ & 0,22 & 0,03 & $<$ D.L. & $<$ D.L. & $<$ D.L. & 0,04 & 0,03 & 0,05 & 0,12 & 0,13 & 0,01 & 0,05 & 0,04 & 0,08 \\
\hline $\mathrm{Na} 2 \mathrm{O}$ & 0,02 & $<$ D.L. & 0,02 & 0,11 & 0,21 & 0,03 & 0,40 & 0,04 & 0,02 & 1,17 & $<$ D.L. & 0,03 & 0,31 & 0,28 \\
\hline $\mathrm{K} 2 \mathrm{O}$ & 0,17 & $<$ D.L. & $<$ D.L. & 10,42 & 10,82 & 0,74 & 10,12 & 0,54 & 0,11 & 9,13 & 0,02 & 0,39 & 10,61 & 10,46 \\
\hline Total & 87,90 & 88,32 & 87,61 & 94,88 & 96,38 & 87,79 & 97,26 & 87,29 & 88,83 & 95,25 & 88,87 & 88,21 & 96,20 & 96,12 \\
\hline \multicolumn{15}{|l|}{ Cations } \\
\hline $\mathrm{Si}$ & 2,95 & 2,86 & 2,70 & 3,23 & 3,19 & 2,85 & 3,17 & 2,84 & 2,89 & 3,13 & 2,70 & 2,82 & 3,11 & 3,25 \\
\hline $\mathrm{Ti}$ & 0,01 & 0,00 & 0,02 & 0,00 & 0,00 & 0,00 & 0,00 & 0,01 & 0,00 & 0,02 & 0,00 & 0,00 & 0,01 & 0,01 \\
\hline $\mathrm{Al}$ & 2,20 & 2,42 & 2,72 & 2,59 & 2,61 & 2,87 & 2,66 & 2,90 & 2,69 & 2,73 & 2,91 & 2,76 & 2,73 & 2,52 \\
\hline FeTOT & 2,49 & 1,94 & 2,58 & 0,06 & 0,07 & 1,82 & 0,14 & 2,02 & 1,74 & 0,06 & 2,16 & 1,97 & 0,10 & 0,14 \\
\hline $\mathrm{Mn}$ & 0,04 & 0,03 & 0,02 & 0,00 & 0,00 & 0,03 & 0,00 & 0,03 & 0,02 & 0,00 & 0,02 & 0,02 & 0,00 & 0,00 \\
\hline $\mathrm{Mg}$ & 2,21 & 2,66 & 1,88 & 0,12 & 0,16 & 2,10 & 0,07 & 1,88 & 2,40 & 0,09 & 2,06 & 2,19 & 0,08 & 0,09 \\
\hline $\mathrm{Ca}$ & 0,03 & 0,00 & 0,00 & 0,00 & 0,00 & 0,00 & 0,00 & 0,01 & 0,01 & 0,01 & 0,00 & 0,01 & 0,00 & 0,01 \\
\hline $\mathrm{Na}$ & 0,00 & 0,00 & 0,00 & 0,01 & 0,03 & 0,01 & 0,05 & 0,01 & 0,00 & 0,15 & $-0,01$ & 0,01 & 0,04 & 0,04 \\
\hline $\mathrm{K}$ & 0,02 & 0,00 & 0,00 & 0,88 & 0,91 & 0,10 & 0,84 & 0,07 & 0,01 & 0,77 & 0,00 & 0,05 & 0,89 & 0,88 \\
\hline Oxygens & 14 & 14 & 14 & 11 & 11 & 14 & 11 & 14 & 14 & 11 & 14 & 14 & 11 & 11 \\
\hline$X M g$ & 0,47 & 0,52 & 0,42 & 0,65 & 0,68 & 0,54 & 0,33 & 0,48 & 0,58 & 0,60 & 0,49 & 0,53 & 0,47 & 0,39 \\
\hline AlVI & 1,16 & 1,29 & 1,44 & - & - & 1,74 & - & 1,74 & 1,58 & - & 1,61 & 1,59 & - & - \\
\hline Octahedral sum & 5,90 & 5,92 & 5,92 & - & - & 5,69 & - & 5,66 & 5,74 & - & 5,85 & 5,76 & - & - \\
\hline Xceladonite & - & - & - & 0,17 & 0,19 & - & 0,15 & - & - & 0,13 & - & - & 0,14 & 0,21 \\
\hline Xmuscovite & - & - & - & 0,72 & 0,73 & - & 0,71 & - & - & 0,66 & - & - & 0,77 & 0,68 \\
\hline Xpyrophyllite & - & - & - & 0,10 & 0,06 & - & 0,10 & - & - & 0,07 & - & - & 0,06 & 0,07 \\
\hline
\end{tabular}


Table S2. Representative chemical composition of dated monazite for samples ZA16-98 and ZAL18-11.

Page 64 of 203

SESSION 1

\begin{tabular}{|c|c|c|c|c|c|c|c|c|c|c|c|c|c|c|c|c|c|}
\hline $\begin{array}{c}\text { ZA16-98 } \\
\text { No. }\end{array}$ & $\begin{array}{r}\mathbf{W t} \% \\
\mathrm{CaO} \\
\end{array}$ & $\mathrm{SiO2}$ & P2O5 & Y2O3 & Dy203 & Gd2O3 & Sm2O3 & Pr203 & $\mathrm{Nd} 2 \mathrm{O} 3$ & $\mathrm{Ce} 2 \mathrm{O3}$ & La2O3 & ThO2 & UO2 & $\mathrm{PbO}$ & TOTAL & Age (Ma) & std \\
\hline D.L. (ppm) & 99 & 58 & 244 & 217 & 723 & 626 & 559 & 520 & 341 & 391 & 415 & 130 & 136 & 93 & & & \\
\hline za16-98_Mz33 & 1,19 & 0,86 & 27,83 & 0,45 & 0,20 & 0,59 & 1,14 & 3,29 & 10,12 & 34,62 & 15,59 & 7,65 & 0,15 & 0,06 & 103,75 & 188 & 6 \\
\hline za16-98_Mz34 & 1,15 & 0,70 & 28,49 & 0,43 & 0,15 & 0,63 & 1,23 & 3,30 & 10,36 & 33,83 & 16,06 & 7,38 & 0,16 & 0,07 & 103,96 & 212 & 6 \\
\hline za16-98_Mz35 & 0,64 & 1,49 & 28,30 & 0,32 & 0,20 & 0,54 & 1,09 & 3,31 & 10,19 & 35,68 & 16,89 & 2,39 & 0,06 & 0,02 & 101,12 & 227 & 18 \\
\hline za16-98_Mz36 & 0,70 & 1,54 & 27,82 & 0,47 & 0,23 & 0,71 & 1,29 & 3,20 & 9,98 & 33,86 & 16,37 & 2,36 & 0,10 & 0,02 & 98,67 & 210 & 17 \\
\hline za16-98_Mz38 & 1,08 & 1,11 & 27,85 & 0,46 & 0,18 & 0,52 & 0,92 & 3,13 & 9,18 & 35,18 & 18,04 & 3,69 & 0,18 & 0,04 & 101,56 & 227 & 11 \\
\hline za16-98_Mz39 & 0,59 & 0,50 & 29,18 & 0,44 & 0,27 & 0,78 & 1,27 & 3,20 & 10,24 & 35,00 & 17,89 & 1,59 & 0,06 & 0,01 & 101,00 & 150 & 26 \\
\hline za16-98_Mz40 & 0,84 & 0,26 & 27,53 & 0,59 & 0,23 & 0,54 & 0,96 & 3,29 & 9,84 & 37,36 & 18,52 & 1,13 & 0,08 & 0,01 & 101,20 & 253 & 33 \\
\hline za16-98_Mz41 & 0,70 & 0,54 & 29,32 & 0,76 & 0,30 & 0,72 & 1,09 & 3,12 & 9,66 & 34,40 & 17,78 & 1,88 & 0,11 & 0,02 & 100,40 & 190 & 20 \\
\hline za16-98_Mz42 & 0,55 & 0,36 & 28,10 & 1,13 & 0,43 & 0,95 & 1,30 & 3,27 & 10,39 & 35,40 & 18,35 & 0,29 & 0,13 & 0,01 & 100,67 & 353 & 62 \\
\hline za16-98_Mnz1 & 1,23 & 1,23 & 26,94 & 0,42 & 0,25 & 0,51 & 1,07 & 3,15 & 9,74 & 33,57 & 15,02 & 7,77 & 0,16 & 0,06 & 101,11 & 185 & 6 \\
\hline za16-98_Mnz1-2 & 1,13 & 1,02 & 27,34 & 0,43 & 0,18 & 0,62 & 1,19 & 3,14 & 9,89 & 32,85 & 15,38 & 7,17 & 0,14 & 0,06 & 100,54 & 186 & 6 \\
\hline za16-98_Mnz2-2 & 0,73 & 0,36 & 28,35 & 0,61 & 0,27 & 0,72 & 1,26 & 3,39 & 10,45 & 36,07 & 16,39 & 1,45 & 0,07 & 0,02 & 100,13 & 225 & 28 \\
\hline za16-98_Mz3 & 0,43 & 0,27 & 29,82 & 1,05 & 0,52 & 1,51 & 2,21 & 3,36 & 11,57 & 33,35 & 18,45 & 0,00 & 0,05 & $<$ D.L. & 102,61 & 88 & 9 \\
\hline za16-98_Mz7 & 0,79 & 0,17 & 29,61 & 1,16 & 0,51 & 1,62 & 2,23 & 3,39 & 11,94 & 33,09 & 17,39 & 0,01 & 0,05 & $<$ D.L. & 101,93 & 275 & 47 \\
\hline za16-98_Mz9 & 0,72 & 0,14 & 28,58 & 0,76 & 0,36 & 0,85 & 1,40 & 3,41 & 10,74 & 36,89 & 16,51 & 0,82 & 0,10 & 0,01 & 101,30 & 204 & 40 \\
\hline za16-98_Mz10 & 0,90 & 1,38 & 27,41 & 0,44 & 0,28 & 0,70 & 1,30 & 3,27 & 10,23 & 34,14 & 15,76 & 4,29 & 0,09 & 0,05 & 100,24 & 247 & 10 \\
\hline za16-98_Mz15 & 0,79 & 2,58 & 27,73 & 0,40 & 0,18 & 0,58 & 1,01 & 3,16 & 9,50 & 34,18 & 17,62 & 2,50 & 0,15 & 0,03 & 100,39 & 218 & 16 \\
\hline za16-98_Mz16 & 0,73 & 0,61 & 28,18 & 0,32 & 0,12 & 0,43 & 0,90 & 3,26 & 9,49 & 36,36 & 17,94 & 1,95 & 0,10 & 0,02 & 100,40 & 205 & 20 \\
\hline za16-98_Mz17 & 1,13 & 0,58 & 21,80 & 0,39 & 0,13 & 0,51 & 0,91 & 3,33 & 9,94 & 38,70 & 19,34 & 3,63 & 0,16 & 0,04 & 100,58 & 256 & 11 \\
\hline za16-98_Mz18 & 0,85 & 0,72 & 28,43 & 0,45 & 0,21 & 0,58 & 1,00 & 3,29 & 9,79 & 35,67 & 18,10 & 2,75 & 0,15 & 0,03 & 102,02 & 237 & 14 \\
\hline za16-98_Mz23 & 0,93 & 0,22 & 28,06 & 0,58 & 0,26 & 0,55 & 1,01 & 3,20 & 9,49 & 36,10 & 18,21 & 2,15 & 0,11 & 0,02 & 100,90 & 193 & 19 \\
\hline za16-98_Mz24 & 0,74 & 0,31 & 28,83 & 0,66 & 0,23 & 0,63 & 1,07 & 3,19 & 9,57 & 35,67 & 18,63 & 1,75 & 0,08 & 0,01 & 101,39 & 106 & 23 \\
\hline za16-98_Mz25 & 0,58 & 0,12 & 28,74 & 0,70 & 0,29 & 0,79 & 1,24 & 3,30 & 10,26 & 35,85 & 18,75 & 1,29 & 0,11 & 0,02 & 102,04 & 271 & 29 \\
\hline za16-98_Mz26 & 0,60 & 0,02 & 29,52 & 1,68 & 0,50 & 1,14 & 1,38 & 3,14 & 9,99 & 34,48 & 20,04 & 0,65 & 0,12 & 0,02 & 103,27 & 438 & 42 \\
\hline za16-98_Mz27 & 0,53 & 0,02 & 30,28 & 1,44 & 0,58 & 1,13 & 1,39 & 3,06 & 10,07 & 33,16 & 19,17 & 0,58 & 0,09 & 0,00 & 101,51 & 47 & 3 \\
\hline za16-98_Mz28 & 0,75 & 0,42 & 28,55 & 0,50 & 0,16 & 0,53 & 1,00 & 3,17 & 9,44 & 35,41 & 17,91 & 2,28 & 0,08 & 0,03 & 100,22 & 255 & 19 \\
\hline
\end{tabular}


SESSION 2

\begin{tabular}{|c|c|c|c|c|c|c|c|c|c|c|c|c|c|c|c|c|c|}
\hline Sample & ZAL18-11 & & & & & & & & & & & & & & & & \\
\hline No. & $\mathrm{CaO}$ & $\mathrm{SiO2}$ & P2O5 & Y2O3 & Dy203 & Gd2O3 & Sm2O3 & Pr203 & $\mathrm{Nd} 2 \mathrm{O} 3$ & $\mathrm{Ce} 2 \mathrm{O} 3$ & La2O3 & ThO2 & UO2 Corr & $\mathrm{PbO}$ & TOTAL & Age (Ma) & Std \\
\hline D.L. (ppm) & 101 & 41 & 288 & 228 & 729 & 649 & 563 & 527 & 341 & 385 & 414 & 141 & 142 & 101 & & & \\
\hline ZAL18-11-Mnz3 & 1,07 & 0,22 & 30,13 & 2,01 & 0,59 & 1,43 & 1,83 & 3,23 & 12,30 & 29,29 & 14,75 & 4,45 & 0,72 & 0,07 & 102,10 & 259 & 8 \\
\hline ZAL18-11-Mnz1-5 & 0,28 & 0,52 & 29,28 & 0,25 & 0,20 & 1,40 & 2,09 & 3,39 & 13,40 & 33,52 & 14,58 & 2,95 & 0,04 & 0,04 & 101,93 & 275 & 16 \\
\hline ZAL18-11-Mnz2 & 0,60 & 0,20 & 30,49 & 1,45 & 0,59 & 1,45 & 1,95 & 3,32 & 12,73 & 30,60 & 15,50 & 2,75 & 0,37 & 0,05 & 102,04 & 325 & 12 \\
\hline ZAL18-11-Mnz3-2 & 0,30 & 0,22 & 29,53 & 0,25 & 0,20 & 0,97 & 1,76 & 3,30 & 12,29 & 34,37 & 16,19 & 1,50 & 0,01 & 0,01 & 100,92 & 203 & 32 \\
\hline ZAL18-11-Mnz3-3 & 0,55 & 0,35 & 29,28 & 0,29 & 0,16 & 1,09 & 2,18 & 3,49 & 14,69 & 32,33 & 13,79 & 2,96 & 0,06 & 0,03 & 101,24 & 212 & 16 \\
\hline ZAL18-11-Mnz3-4 & 0,55 & 0,14 & 29,75 & 0,40 & 0,19 & 1,11 & 1,81 & 3,22 & 12,20 & 33,16 & 17,35 & 1,18 & 0,03 & 0,01 & 101,10 & 204 & 39 \\
\hline ZAL18-11-Mnz3-6 & 0,64 & 0,25 & 30,02 & 1,19 & 0,49 & 1,47 & 1,78 & 3,11 & 11,65 & 33,03 & 15,21 & 3,08 & 0,30 & 0,04 & 102,28 & 258 & 12 \\
\hline ZAL18-11-Mnz3-7 & 0,33 & 0,38 & 29,14 & 0,24 & 0,20 & 0,96 & 1,83 & 3,39 & 12,83 & 33,68 & 15,52 & 2,56 & 0,04 & 0,03 & 101,13 & 229 & 18 \\
\hline ZAL18-11-Mnz3-8 & 0,38 & 0,29 & 29,72 & 1,06 & 0,39 & 1,15 & 1,51 & 3,05 & 11,37 & 33,73 & 16,17 & 2,39 & 0,13 & 0,03 & 101,39 & 235 & 17 \\
\hline ZAL18-11-Mnz3-9 & 0,99 & 0,31 & 29,62 & 0,72 & 0,37 & 1,45 & 1,96 & 3,17 & 12,83 & 31,96 & 13,25 & 4,99 & 0,26 & 0,06 & 101,91 & 232 & 9 \\
\hline ZAL18-11-Mnz3-10 & 0,53 & 0,19 & 29,83 & 0,75 & 0,26 & 1,07 & 1,71 & 3,22 & 11,99 & 33,62 & 16,63 & 1,44 & 0,05 & 0,01 & 101,30 & 209 & 31 \\
\hline ZAL18-11-Mnz3-11 & 0,39 & 0,20 & 29,93 & 1,07 & 0,35 & 0,98 & 1,42 & 3,06 & 11,39 & 33,85 & 17,16 & 1,85 & 0,14 & 0,02 & 101,80 & 200 & 22 \\
\hline ZAL18-11-Mnz3-12 & 1,04 & 0,28 & 29,88 & 0,39 & 0,23 & 1,20 & 1,90 & 3,23 & 12,62 & 32,06 & 14,17 & 4,74 & 0,18 & 0,06 & 101,97 & 270 & 10 \\
\hline ZAL18-11-Mnz5-3 & 0,59 & 2,85 & 27,91 & 0,31 & 0,13 & 0,95 & 1,24 & 2,86 & 10,55 & 30,48 & 17,04 & 0,63 & 0,01 & 0,01 & 95,59 & 403 & 72 \\
\hline ZAL18-11-Mnz6-1 & 2,54 & 0,34 & 28,50 & 1,71 & 0,52 & 1,37 & 1,99 & 3,08 & 11,34 & 27,18 & 12,89 & 8,53 & 2,14 & 0,06 & 102,18 & 90 & 3 \\
\hline ZAL18-11-Mnz8-1 & 0,66 & 0,69 & 29,83 & 1,32 & 0,47 & 1,35 & 2,15 & 3,36 & 13,36 & 30,23 & 14,43 & 1,85 & 0,43 & 0,03 & 100,15 & 197 & 16 \\
\hline ZAL18-11-Mnz8-2 & 1,18 & 1,31 & 29,93 & 0,84 & 0,32 & 1,31 & 2,24 & 3,35 & 13,19 & 29,16 & 13,50 & 3,51 & 0,84 & 0,06 & 100,75 & 243 & 8 \\
\hline ZAL18-11-Mnz9-1 & 0,96 & 0,18 & 29,78 & 0,65 & 0,32 & 1,44 & 2,58 & 3,54 & 14,75 & 30,17 & 13,18 & 1,60 & 0,18 & 0,02 & 99,34 & 185 & 23 \\
\hline ZAL18-11-Mnz9-6 & 0,95 & 1,41 & 29,85 & 0,40 & 0,24 & 1,21 & 2,16 & 3,40 & 13,48 & 31,40 & 14,61 & 1,09 & 0,08 & 0,01 & 100,30 & 180 & 37 \\
\hline ZAL18-11-Mnz6 & 2,84 & 0,34 & 29,14 & 1,76 & 0,56 & 1,49 & 2,03 & 3,08 & 11,54 & 26,68 & 12,22 & 7,61 & 0,98 & 0,12 & 100,37 & 90 & 3 \\
\hline ZAL18-11-Mnz6-3 & 2,84 & 0,32 & 29,87 & 1,76 & 0,55 & 1,51 & 2,14 & 3,27 & 11,99 & 27,93 & 12,84 & 7,40 & 0,57 & 0,06 & 103,03 & 160 & 6 \\
\hline ZAL18-11-Mnz6-4 & 2,93 & 0,16 & 29,84 & 1,15 & 0,41 & 1,43 & 2,16 & 3,20 & 12,19 & 29,25 & 15,04 & 4,69 & 0,70 & 0,07 & 103,21 & 234 & 7 \\
\hline
\end{tabular}




\begin{tabular}{|c|c|c|c|c|c|c|}
\hline Sample & ${ }^{207} \mathrm{~Pb} /{ }^{235} \mathrm{U}$ & Error $2 \sigma$ & ${ }^{206} \mathrm{~Pb} /{ }^{238} \mathrm{U}$ & Error $2 \sigma$ & orrelation_6_38i & ${ }^{238} \mathrm{U} /{ }^{206} \mathrm{~Pb}$ \\
\hline T_MKED1_1 & 3.830 & 0.200 & 0.271 & 0.006 & 0.185 & 3.685 \\
\hline T_MKED1_2 & 3.469 & 0.067 & 0.262 & 0.005 & 0.295 & 3.824 \\
\hline T_MKED1_3 & 3.830 & 0.550 & 0.270 & 0.006 & -0.010 & 3.708 \\
\hline T_MKED1_4 & 3.480 & 0.074 & 0.263 & 0.005 & 0.795 & 3.797 \\
\hline T_MKED1_5 & 3.452 & 0.068 & 0.269 & 0.006 & 0.266 & 3.723 \\
\hline T_MKED1_6 & 3.387 & 0.068 & 0.258 & 0.005 & 0.630 & 3.880 \\
\hline T_MKED1_7 & 3.497 & 0.080 & 0.263 & 0.005 & 0.276 & 3.804 \\
\hline T_MKED1_8 & 3.534 & 0.091 & 0.267 & 0.006 & 0.859 & 3.751 \\
\hline T_MKED1_9 & 3.400 & 0.110 & 0.261 & 0.004 & 0.227 & 3.837 \\
\hline T_MKED1_10 & 3.580 & 0.110 & 0.265 & 0.006 & 0.505 & 3.771 \\
\hline T_MKED1_11 & 3.389 & 0.094 & 0.260 & 0.005 & 0.346 & 3.849 \\
\hline T_MKED1_12 & 3.605 & 0.089 & 0.272 & 0.006 & 0.262 & 3.679 \\
\hline T_MKED1_13 & 3.620 & 0.082 & 0.273 & 0.005 & 0.664 & 3.662 \\
\hline T_MKED1_14 & 3.740 & 0.110 & 0.272 & 0.005 & 0.078 & 3.674 \\
\hline T_MKED1_15 & 3.680 & 0.110 & 0.281 & 0.007 & 0.475 & 3.557 \\
\hline T_MKED1_16 & 3.570 & 0.130 & 0.269 & 0.005 & 0.673 & 3.720 \\
\hline T_MKED1_17 & 3.650 & 0.110 & 0.274 & 0.006 & 0.596 & 3.650 \\
\hline T_MKED1_18 & 3.491 & 0.076 & 0.265 & 0.007 & 0.648 & 3.774 \\
\hline T_MKED1_19 & 3.570 & 0.160 & 0.265 & 0.006 & 0.810 & 3.774 \\
\hline T_MKED1_20 & 3.570 & 0.150 & 0.268 & 0.006 & 0.010 & 3.727 \\
\hline T_MKED1_21 & 3.370 & 0.140 & 0.257 & 0.006 & 0.381 & 3.894 \\
\hline T_MKED1_22 & 3.641 & 0.092 & 0.279 & 0.006 & 0.672 & 3.583 \\
\hline T_MKED1_23 & 3.650 & 0.120 & 0.274 & 0.007 & 0.963 & 3.652 \\
\hline T_MKED1_24 & 3.880 & 0.270 & 0.269 & 0.007 & 0.013 & 3.717 \\
\hline T_MKED1_25 & 3.650 & 0.240 & 0.264 & 0.007 & 0.842 & 3.782 \\
\hline T_MKED1_26 & 4.800 & 1.200 & 0.272 & 0.007 & 0.446 & 3.682 \\
\hline T_MKED1_27 & 3.700 & 0.260 & 0.259 & 0.005 & 0.876 & 3.867 \\
\hline T_MKED1_28 & 3.590 & 0.100 & 0.268 & 0.006 & 0.406 & 3.736 \\
\hline T_MKED1_29 & 3.682 & 0.089 & 0.277 & 0.006 & 0.148 & 3.614 \\
\hline T_MKED1_30 & 3.360 & 0.110 & 0.259 & 0.007 & 0.426 & 3.864 \\
\hline T_MKED1_31 & 4.040 & 0.290 & 0.285 & 0.008 & 0.462 & 3.506 \\
\hline T_MKED1_32 & 3.680 & 0.100 & 0.278 & 0.008 & 0.439 & 3.604 \\
\hline T_MKED1_33 & 3.448 & 0.095 & 0.262 & 0.005 & 0.351 & 3.811 \\
\hline T_MKED1_34 & 4.080 & 0.330 & 0.282 & 0.012 & 0.567 & 3.546 \\
\hline T_MKED1_35 & 3.990 & 0.330 & 0.274 & 0.007 & 0.317 & 3.647 \\
\hline T_MKED1_36 & 3.590 & 0.150 & 0.272 & 0.006 & 0.272 & 3.681 \\
\hline X16_ttn_1 & 429.000 & 36.000 & 3.720 & 0.300 & 0.963 & 0.269 \\
\hline X16_ttn_2 & 329.000 & 28.000 & 2.880 & 0.250 & 0.989 & 0.347 \\
\hline X16_ttn_3 & 154.000 & 25.000 & 1.320 & 0.180 & 0.715 & 0.758 \\
\hline X16_ttn_4 & 601.000 & 38.000 & 5.270 & 0.320 & 0.966 & 0.190 \\
\hline X16_ttn_5 & 104.000 & 11.000 & 0.938 & 0.098 & 0.975 & 1.066 \\
\hline X16_ttn_6 & 237.000 & 28.000 & 2.120 & 0.250 & 0.983 & 0.472 \\
\hline X16_ttn_7 & 40.900 & 3.500 & 0.410 & 0.033 & 0.564 & 2.439 \\
\hline X16_ttn_8 & 1360.000 & 120.000 & 11.900 & 1.100 & 0.985 & 0.084 \\
\hline X16_ttn_9 & 758.000 & 51.000 & 6.630 & 0.450 & 0.817 & 0.151 \\
\hline X16_ttn_10 & 268.000 & 14.000 & 2.330 & 0.130 & 0.348 & 0.429 \\
\hline X16_ttn_11 & 181.000 & 13.000 & 1.595 & 0.092 & 0.940 & 0.627 \\
\hline X16_ttn_12 & 1700.000 & 210.000 & 14.300 & 1.700 & 0.990 & 0.070 \\
\hline
\end{tabular}




\begin{tabular}{|c|c|c|c|c|c|c|}
\hline X16_ttn_13 & 181.000 & 29.000 & 1.630 & 0.270 & 0.992 & 0.613 \\
\hline X16_ttn_14 & 288.000 & 12.000 & 2.550 & 0.120 & 0.910 & 0.392 \\
\hline X16_ttn_15 & 1359.000 & 47.000 & 11.680 & 0.410 & 0.915 & 0.086 \\
\hline X16_ttn_16 & 1497.000 & 54.000 & 12.960 & 0.500 & 0.943 & 0.077 \\
\hline X16_ttn_17 & 1660.000 & 130.000 & 14.500 & 1.300 & 0.249 & 0.069 \\
\hline X16_ttn_18 & 2120.000 & 250.000 & 18.500 & 2.200 & 0.993 & 0.054 \\
\hline X16_ttn_19 & 1519.000 & 59.000 & 12.970 & 0.640 & 0.015 & 0.077 \\
\hline X16_ttn_20 & 9010.000 & 510.000 & 77.800 & 4.400 & 0.983 & 0.013 \\
\hline X16_ttn_21 & 349.000 & 20.000 & 3.070 & 0.180 & 0.990 & 0.326 \\
\hline X16_ttn_22 & 142.000 & 13.000 & 1.290 & 0.110 & 0.978 & 0.775 \\
\hline X16_ttn_23 & 174.000 & 24.000 & 1.430 & 0.180 & 0.848 & 0.699 \\
\hline X16_ttn_24 & 52.200 & 3.700 & 0.501 & 0.034 & 0.857 & 1.996 \\
\hline X16_ttn_25 & 46.500 & 1.500 & 0.454 & 0.014 & 0.791 & 2.203 \\
\hline X16_ttn_26 & 113.600 & 3.400 & 1.024 & 0.033 & 0.926 & 0.977 \\
\hline X16_ttn_27 & 111.200 & 3.900 & 0.989 & 0.037 & 0.904 & 1.011 \\
\hline X16_ttn_28 & 4350.000 & 900.000 & 41.400 & 9.500 & 0.607 & 0.024 \\
\hline X16_ttn_29 & 1757.000 & 72.000 & 14.900 & 0.650 & 0.946 & 0.067 \\
\hline X16_ttn_30 & 1850.000 & 150.000 & 16.000 & 1.200 & 0.987 & 0.063 \\
\hline X16_ttn_31 & 5390.000 & 510.000 & 46.400 & 4.500 & 0.987 & 0.022 \\
\hline X16_ttn_32 & 138.000 & 19.000 & 1.220 & 0.170 & 0.973 & 0.820 \\
\hline X16_ttn_33 & 1159.000 & 76.000 & 9.960 & 0.680 & 0.951 & 0.100 \\
\hline X16_ttn_34 & -25000000.000 & \#\#\#\#\#\#\#\# & -180000.000 & 130000.000 & 0.997 & 0.000 \\
\hline X16_ttn_35 & 1051.000 & 96.000 & 8.500 & 0.670 & 0.844 & 0.118 \\
\hline X16_ttn_36 & 4080.000 & 670.000 & 33.800 & 5.400 & 0.998 & 0.030 \\
\hline X16_ttn_37 & 8.000 & 1.700 & 0.118 & 0.014 & 0.956 & 8.475 \\
\hline X16_ttn_38 & 143.900 & 9.400 & 1.285 & 0.078 & 0.922 & 0.778 \\
\hline X16_ttn_39 & 3.050 & 0.160 & 0.075 & 0.002 & 0.139 & 13.369 \\
\hline X16_ttn_40 & 151.000 & 10.000 & 1.341 & 0.082 & 0.882 & 0.746 \\
\hline X16_ttn_41 & 294.000 & 29.000 & 2.580 & 0.260 & 0.987 & 0.388 \\
\hline X16_ttn_42 & 220.000 & 11.000 & 1.930 & 0.086 & 0.978 & 0.518 \\
\hline X82_ttn_1 & 1020.000 & 190.000 & 8.900 & 1.600 & 0.997 & 0.112 \\
\hline X82_ttn_2 & 256.000 & 20.000 & 2.260 & 0.180 & 0.980 & 0.442 \\
\hline X82_ttn_3 & 71.100 & 4.300 & 0.685 & 0.045 & 0.986 & 1.460 \\
\hline X82_ttn_4 & 274.000 & 36.000 & 2.450 & 0.300 & 0.990 & 0.408 \\
\hline X82_ttn_5 & 12800.000 & 2500.000 & 110.000 & 23.000 & 0.993 & 0.009 \\
\hline X82_ttn_6 & 8200.000 & 1000.000 & 73.600 & 9.700 & 0.900 & 0.014 \\
\hline X82_ttn_7 & 356.000 & 76.000 & 3.100 & 0.660 & 0.986 & 0.323 \\
\hline X82_ttn_8 & 258.000 & 22.000 & 2.240 & 0.170 & 0.859 & 0.446 \\
\hline X82_ttn_9 & 187.000 & 19.000 & 1.700 & 0.170 & 0.987 & 0.588 \\
\hline X82_ttn_10 & 459.000 & 18.000 & 3.970 & 0.150 & 0.941 & 0.252 \\
\hline X82_ttn_11 & 627.000 & 54.000 & 5.560 & 0.490 & 0.046 & 0.180 \\
\hline X82_ttn_12 & 47000.000 & \#\#\#\#\#\#\#\# & 384.000 & 75.000 & 0.983 & 0.003 \\
\hline X82_ttn_13 & 3.420 & 0.350 & 0.080 & 0.005 & 0.913 & 12.516 \\
\hline X82_ttn_14 & 1.996 & 0.043 & 0.067 & 0.002 & 0.289 & 15.015 \\
\hline X82_ttn_15 & 7.690 & 0.240 & 0.116 & 0.003 & 0.826 & 8.591 \\
\hline X82_ttn_16 & 33.000 & 3.000 & 0.337 & 0.027 & 0.934 & 2.967 \\
\hline X82_ttn_17 & 5.860 & 0.350 & 0.103 & 0.004 & 0.908 & 9.709 \\
\hline X82_ttn_18 & 5.180 & 0.490 & 0.093 & 0.005 & 0.884 & 10.753 \\
\hline X82_ttn_19 & 8.000 & 1.300 & 0.111 & 0.009 & 0.601 & 9.050 \\
\hline
\end{tabular}




\begin{tabular}{|c|c|c|c|c|c|c|}
\hline X82_ttn_20 & 15.600 & 0.980 & 0.184 & 0.008 & 0.927 & 5.441 \\
\hline X82_ttn_21 & 137.000 & 13.000 & 1.200 & 0.110 & 0.899 & 0.833 \\
\hline X82_ttn_22 & 6.260 & 0.240 & 0.107 & 0.004 & 0.852 & 9.346 \\
\hline X82_ttn_23 & 2.890 & 0.130 & 0.073 & 0.003 & 0.762 & 13.680 \\
\hline X82_ttn_24 & 7.290 & 0.300 & 0.115 & 0.003 & 0.514 & 8.688 \\
\hline X82_ttn_25 & 4.400 & 0.430 & 0.086 & 0.006 & 0.916 & 11.601 \\
\hline X82_ttn_26 & 77.100 & 4.700 & 0.712 & 0.040 & 0.985 & 1.404 \\
\hline X82_ttn_27 & 107.900 & 7.200 & 0.997 & 0.064 & 0.886 & 1.003 \\
\hline X82_ttn_28 & 144.000 & 12.000 & 1.340 & 0.120 & 0.599 & 0.746 \\
\hline X82_ttn_29 & 18.200 & 1.300 & 0.208 & 0.018 & 0.535 & 4.808 \\
\hline X82_ttn_30 & 17.820 & 0.780 & 0.204 & 0.009 & 0.887 & 4.912 \\
\hline X82_ttn_31 & 57.500 & 5.100 & 0.553 & 0.046 & 0.973 & 1.808 \\
\hline X82_ttn_32 & 175.100 & 6.000 & 1.564 & 0.052 & 0.941 & 0.639 \\
\hline X82_ttn_33 & 432.000 & 19.000 & 3.760 & 0.160 & 0.882 & 0.266 \\
\hline X82_ttn_34 & 223.900 & 5.700 & 2.006 & 0.051 & 0.882 & 0.499 \\
\hline X82_ttn_35 & 169.500 & 9.800 & 1.507 & 0.083 & 0.770 & 0.664 \\
\hline X82_ttn_36 & 205.900 & 7.000 & 1.801 & 0.048 & 0.373 & 0.555 \\
\hline X82_ttn_37 & 319.000 & 29.000 & 2.990 & 0.320 & 0.610 & 0.334 \\
\hline X82_ttn_38 & 236.000 & 12.000 & 2.010 & 0.093 & 0.523 & 0.498 \\
\hline X82_ttn_39 & 355.000 & 13.000 & 3.050 & 0.110 & 0.931 & 0.328 \\
\hline X82_ttn_40 & 200.000 & 11.000 & 1.743 & 0.082 & 0.961 & 0.574 \\
\hline X82_ttn_41 & 243.000 & 11.000 & 2.180 & 0.100 & 0.913 & 0.459 \\
\hline X82_ttn_42 & 131.500 & 9.800 & 1.330 & 0.220 & 0.203 & 0.752 \\
\hline X82_ttn_43 & 75.100 & 6.200 & 0.670 & 0.049 & 0.147 & 1.493 \\
\hline X82_ttn_44 & 207.200 & 8.400 & 1.805 & 0.074 & 0.921 & 0.554 \\
\hline X82_ttn_45 & 1019.000 & 83.000 & 8.990 & 0.720 & 0.980 & 0.111 \\
\hline X82_ttn_46 & 75.700 & 3.100 & 0.678 & 0.021 & 0.052 & 1.475 \\
\hline X82_ttn_47 & 452.000 & 16.000 & 3.880 & 0.140 & 0.655 & 0.258 \\
\hline X82_ttn_48 & 122.800 & 6.400 & 1.084 & 0.040 & 0.343 & 0.923 \\
\hline X82_ttn_49 & 226.000 & 36.000 & 1.940 & 0.290 & 0.982 & 0.515 \\
\hline X82_ttn_50 & 95.600 & 3.500 & 0.857 & 0.030 & 0.859 & 1.167 \\
\hline X82_ttn_51 & 330.000 & 26.000 & 2.890 & 0.220 & 0.961 & 0.346 \\
\hline X82_ttn_52 & 166.500 & 9.800 & 1.540 & 0.110 & 0.911 & 0.649 \\
\hline X82_ttn_53 & 530.000 & 84.000 & 4.420 & 0.680 & 0.768 & 0.226 \\
\hline X82_ttn_54 & 771.000 & 51.000 & 6.740 & 0.400 & 0.959 & 0.148 \\
\hline X82_ttn_55 & 360.000 & 13.000 & 3.190 & 0.140 & 0.317 & 0.313 \\
\hline X82_ttn_56 & 29.600 & 2.100 & 0.293 & 0.017 & 0.870 & 3.413 \\
\hline X82_ttn_57 & 190.000 & 36.000 & 1.590 & 0.300 & 0.988 & 0.629 \\
\hline X82_ttn_58 & 33.500 & 3.200 & 0.333 & 0.028 & 0.961 & 3.003 \\
\hline X82_ttn_59 & 39.100 & 2.700 & 0.402 & 0.028 & 0.739 & 2.488 \\
\hline X82_ttn_60 & 144.000 & 27.000 & 1.200 & 0.190 & 0.998 & 0.833 \\
\hline X82_ttn_61 & 168.000 & 14.000 & 1.500 & 0.100 & 0.901 & 0.667 \\
\hline X82_ttn_62 & 38.700 & 2.100 & 0.393 & 0.022 & 0.165 & 2.545 \\
\hline X84_ttn_1 & 164.000 & 35.000 & 1.590 & 0.360 & 0.994 & 0.629 \\
\hline X84_ttn_2 & 48.900 & 6.200 & 0.455 & 0.047 & -0.059 & 2.198 \\
\hline X84_ttn_3 & 55.600 & 4.800 & 0.542 & 0.044 & 0.296 & 1.845 \\
\hline X84_ttn_4 & 63.400 & 5.700 & 0.583 & 0.044 & 0.695 & 1.715 \\
\hline X84_ttn_5 & 125.000 & 13.000 & 1.110 & 0.120 & 0.931 & 0.901 \\
\hline$x 84 \operatorname{ttn} 6$ & 111.000 & 23.000 & 0.980 & 0.200 & 0.978 & 1.020 \\
\hline
\end{tabular}




\begin{tabular}{|c|c|c|c|c|c|c|}
\hline X84_ttn_7 & 92.600 & 4.600 & 0.862 & 0.045 & 0.703 & 1.160 \\
\hline X84_ttn_8 & 524.000 & 66.000 & 4.550 & 0.570 & 0.982 & 0.220 \\
\hline X84_ttn_9 & 4.140 & 0.890 & 0.082 & 0.007 & 0.038 & 12.255 \\
\hline X84_ttn_10 & 44.400 & 4.500 & 0.420 & 0.036 & 0.061 & 2.381 \\
\hline X84_ttn_11 & 37.000 & 3.300 & 0.362 & 0.033 & 0.852 & 2.762 \\
\hline X84_ttn_12 & 58.000 & 9.000 & 0.537 & 0.078 & 0.965 & 1.862 \\
\hline X84_ttn_13 & 83.300 & 7.200 & 0.755 & 0.057 & 0.712 & 1.325 \\
\hline X84_ttn_14 & 101.000 & 14.000 & 0.880 & 0.110 & 0.306 & 1.136 \\
\hline X84_ttn_15 & 44.700 & 2.800 & 0.442 & 0.023 & 0.255 & 2.262 \\
\hline X84_ttn_16 & 154.600 & 8.500 & 1.420 & 0.170 & 0.884 & 0.704 \\
\hline X84_ttn_17 & 163.000 & 10.000 & 1.482 & 0.095 & 0.189 & 0.675 \\
\hline X84_ttn_18 & 610.000 & 140.000 & 5.600 & 1.300 & 0.982 & 0.179 \\
\hline X84_ttn_19 & 26.200 & 3.900 & 0.286 & 0.033 & 0.556 & 3.497 \\
\hline X84_ttn_20 & 280.000 & 49.000 & 2.550 & 0.400 & 0.768 & 0.392 \\
\hline X84_ttn_21 & 296.000 & 35.000 & 2.510 & 0.290 & 0.658 & 0.398 \\
\hline X84_ttn_22 & 169.000 & 27.000 & 1.367 & 0.078 & 0.243 & 0.732 \\
\hline X84_ttn_23 & 106.700 & 5.900 & 0.995 & 0.094 & 0.917 & 1.005 \\
\hline X84_ttn_24 & 772.000 & 82.000 & 7.500 & 1.200 & 0.360 & 0.133 \\
\hline X84_ttn_25 & 21.400 & 1.500 & 0.236 & 0.013 & 0.338 & 4.237 \\
\hline X84_ttn_26 & 401.000 & 40.000 & 3.570 & 0.360 & 0.346 & 0.280 \\
\hline X84_ttn_27 & 87.900 & 7.100 & 0.800 & 0.100 & 0.536 & 1.250 \\
\hline X84_ttn_28 & 117.000 & 17.000 & 1.240 & 0.240 & 0.455 & 0.806 \\
\hline X84_ttn_29 & 880.000 & 150.000 & 8.000 & 1.500 & 0.954 & 0.125 \\
\hline X84_ttn_30 & 166.000 & 15.000 & 1.405 & 0.063 & 0.037 & 0.712 \\
\hline X84_ttn_31 & 13.140 & 0.850 & 0.161 & 0.008 & 0.885 & 6.219 \\
\hline X84_ttn_32 & 90.100 & 9.800 & 0.823 & 0.082 & 0.653 & 1.215 \\
\hline X84_ttn_33 & 72.000 & 14.000 & 0.750 & 0.150 & 0.640 & 1.333 \\
\hline X84_ttn_34 & 83.400 & 9.900 & 0.776 & 0.095 & 0.458 & 1.289 \\
\hline X84_ttn_35 & 2.110 & 0.190 & 0.065 & 0.002 & 0.422 & 15.432 \\
\hline X84_ttn_36 & 11.300 & 1.500 & 0.144 & 0.014 & 0.257 & 6.944 \\
\hline X84_ttn_37 & 207.000 & 12.000 & 1.810 & 0.110 & -0.128 & 0.552 \\
\hline X84_ttn_38 & 102.000 & 14.000 & 0.940 & 0.120 & 0.104 & 1.064 \\
\hline X84_ttn_39 & 120.000 & 20.000 & 1.340 & 0.740 & 0.899 & 0.746 \\
\hline X84_ttn_40 & 158.800 & 8.800 & 1.423 & 0.067 & 0.627 & 0.703 \\
\hline X84_ttn_41 & 176.000 & 27.000 & 1.460 & 0.200 & 0.707 & 0.685 \\
\hline X84_ttn_42 & 128.200 & 9.100 & 1.196 & 0.073 & 0.876 & 0.836 \\
\hline X84_ttn_43 & 201.000 & 40.000 & 1.640 & 0.290 & 0.376 & 0.610 \\
\hline X84_ttn_44 & 346.000 & 38.000 & 3.800 & 1.100 & 0.445 & 0.263 \\
\hline X117_ttn_1 & 173.000 & 18.000 & 1.680 & 0.190 & 0.272 & 0.595 \\
\hline X117_ttn_2 & 87.000 & 10.000 & 0.796 & 0.081 & 0.994 & 1.256 \\
\hline X117_ttn_3 & 235.100 & 7.500 & 2.127 & 0.066 & 0.901 & 0.470 \\
\hline X117_ttn_4 & 470.000 & 26.000 & 4.070 & 0.230 & 0.984 & 0.246 \\
\hline X117_ttn_5 & 317.500 & 9.100 & 2.850 & 0.100 & 0.321 & 0.351 \\
\hline X117_ttn_6 & 512.000 & 51.000 & 4.290 & 0.390 & 0.776 & 0.233 \\
\hline X117_ttn_7 & 640.000 & 35.000 & 5.640 & 0.300 & 0.979 & 0.177 \\
\hline X117_ttn_8 & 722.000 & 33.000 & 6.400 & 0.300 & 0.972 & 0.156 \\
\hline X117_ttn_9 & 134.600 & 5.400 & 1.234 & 0.040 & 0.464 & 0.810 \\
\hline X117_ttn_10 & 257.600 & 7.700 & 2.335 & 0.068 & 0.206 & 0.428 \\
\hline $\mathrm{X} 117 \mathrm{ttn} 11$ & 378.900 & 8.900 & 3.416 & 0.091 & 0.789 & 0.293 \\
\hline
\end{tabular}




\begin{tabular}{|c|c|c|c|c|c|c|}
\hline X117_ttn_12 & 281.000 & 16.000 & 2.570 & 0.140 & 0.952 & 0.389 \\
\hline X117_ttn_13 & 931.000 & 85.000 & 8.430 & 0.790 & 0.995 & 0.119 \\
\hline X117_ttn_14 & 201.000 & 11.000 & 1.820 & 0.092 & 0.585 & 0.549 \\
\hline X117_ttn_15 & 378.000 & 36.000 & 3.370 & 0.310 & 0.864 & 0.297 \\
\hline X117_ttn_16 & 727.000 & 39.000 & 6.430 & 0.330 & 0.894 & 0.156 \\
\hline X117_ttn_17 & 630.000 & 110.000 & 6.000 & 1.100 & 0.407 & 0.167 \\
\hline X117_ttn_18 & 274.000 & 16.000 & 2.450 & 0.140 & 0.983 & 0.408 \\
\hline X117_ttn_19 & 263.000 & 13.000 & 2.350 & 0.120 & 0.553 & 0.426 \\
\hline X117_ttn_20 & 520.000 & 34.000 & 4.560 & 0.240 & 0.908 & 0.219 \\
\hline X117_ttn_21 & 298.000 & 11.000 & 2.720 & 0.110 & 0.901 & 0.368 \\
\hline X117_ttn_22 & 1164.000 & 47.000 & 10.570 & 0.420 & 0.961 & 0.095 \\
\hline X117_ttn_23 & 414.000 & 29.000 & 3.560 & 0.240 & 0.981 & 0.281 \\
\hline X117_ttn_24 & 224.000 & 10.000 & 2.052 & 0.094 & 0.911 & 0.487 \\
\hline X117_ttn_25 & 262.000 & 14.000 & 2.380 & 0.120 & 0.600 & 0.420 \\
\hline X117_ttn_26 & 261.000 & 20.000 & 2.330 & 0.170 & 0.988 & 0.429 \\
\hline X117_ttn_27 & 283.000 & 31.000 & 2.500 & 0.260 & 0.851 & 0.400 \\
\hline X117_ttn_28 & 132.200 & 7.200 & 1.202 & 0.060 & 0.657 & 0.832 \\
\hline X117_ttn_29 & 753.000 & 26.000 & 6.650 & 0.240 & 0.968 & 0.150 \\
\hline X117_ttn_30 & 1170.000 & 100.000 & 10.480 & 0.950 & 0.986 & 0.095 \\
\hline X117_ttn_31 & 401.000 & 35.000 & 3.460 & 0.280 & 0.945 & 0.289 \\
\hline X117_ttn_32 & 63.300 & 2.700 & 0.594 & 0.026 & 0.733 & 1.684 \\
\hline X117_ttn_33 & 291.000 & 14.000 & 2.600 & 0.130 & 0.337 & 0.385 \\
\hline X117_ttn_34 & 224.800 & 8.300 & 2.023 & 0.079 & 0.911 & 0.494 \\
\hline X117_ttn_35 & 322.000 & 15.000 & 2.840 & 0.130 & 0.978 & 0.352 \\
\hline X117_ttn_36 & 890.000 & 120.000 & 7.900 & 1.000 & 0.992 & 0.127 \\
\hline X117_ttn_37 & 305.000 & 15.000 & 2.680 & 0.140 & 0.440 & 0.373 \\
\hline X117_ttn_38 & 329.000 & 35.000 & 2.920 & 0.300 & 0.991 & 0.342 \\
\hline X117_ttn_39 & 609.000 & 72.000 & 5.120 & 0.590 & 0.969 & 0.195 \\
\hline X117_ttn_40 & 323.000 & 19.000 & 2.860 & 0.150 & 0.966 & 0.350 \\
\hline X117_ttn_41 & 248.000 & 29.000 & 2.240 & 0.260 & 0.969 & 0.446 \\
\hline X117_ttn_42 & 428.000 & 46.000 & 3.710 & 0.390 & 0.987 & 0.270 \\
\hline X117_ttn_43 & 725.000 & 42.000 & 6.720 & 0.400 & 0.908 & 0.149 \\
\hline X117_ttn_44 & 569.000 & 63.000 & 4.830 & 0.460 & 0.949 & 0.207 \\
\hline X117_ttn_45 & 347.000 & 46.000 & 3.100 & 0.390 & 0.969 & 0.323 \\
\hline X117_ttn_46 & 247.000 & 19.000 & 2.210 & 0.170 & 0.878 & 0.452 \\
\hline X117_ttn_47 & 421.000 & 29.000 & 3.710 & 0.260 & 0.981 & 0.270 \\
\hline X117_ttn_48 & 289.000 & 23.000 & 2.640 & 0.220 & 0.975 & 0.379 \\
\hline X117_ttn_49 & 142.600 & 9.100 & 1.317 & 0.080 & 0.847 & 0.759 \\
\hline X117_ttn_50 & 134.900 & 4.400 & 1.241 & 0.040 & 0.961 & 0.806 \\
\hline X117_ttn_51 & 259.000 & 16.000 & 2.300 & 0.140 & 0.959 & 0.435 \\
\hline X117_ttn_52 & 89.700 & 7.800 & 0.853 & 0.075 & 0.985 & 1.172 \\
\hline X117_ttn_53 & 329.000 & 17.000 & 2.840 & 0.120 & 0.550 & 0.352 \\
\hline X117_ttn_54 & 132.500 & 3.200 & 1.207 & 0.041 & 0.587 & 0.829 \\
\hline X117_ttn_55 & 79.400 & 2.900 & 0.748 & 0.019 & -0.023 & 1.337 \\
\hline X117_ttn_56 & 208.800 & 8.600 & 1.879 & 0.076 & 0.722 & 0.532 \\
\hline X117_ttn_57 & 332.000 & 23.000 & 2.970 & 0.200 & 0.988 & 0.337 \\
\hline X117_ttn_58 & 144.000 & 14.000 & 1.310 & 0.130 & 0.778 & 0.763 \\
\hline X117_ttn_59 & 97.700 & 3.800 & 0.911 & 0.037 & 0.688 & 1.098 \\
\hline X117_ttn_60 & 108.000 & 6.600 & 0.963 & 0.046 & 0.499 & 1.038 \\
\hline $\mathrm{X} 117 \mathrm{ttn} 61$ & 103.000 & 4.200 & 0.941 & 0.037 & 0.772 & 1.063 \\
\hline
\end{tabular}




\begin{tabular}{|c|c|c|c|c|c|c|}
\hline X117_ttn_62 & 224.800 & 5.600 & 2.063 & 0.054 & -0.010 & 0.485 \\
\hline X117_ttn_63 & 13.690 & 0.810 & 0.170 & 0.007 & 0.826 & 5.869 \\
\hline X117_ttn_64 & 35.900 & 1.200 & 0.370 & 0.011 & 0.833 & 2.703 \\
\hline X117_ttn_65 & 80.800 & 8.600 & 0.731 & 0.072 & 0.486 & 1.368 \\
\hline X117_ttn_66 & 193.200 & 6.800 & 1.750 & 0.089 & 0.705 & 0.571 \\
\hline X117_ttn_67 & 46.200 & 7.400 & 0.433 & 0.060 & 0.813 & 2.309 \\
\hline X117_ttn_68 & 219.000 & 14.000 & 1.940 & 0.120 & 0.898 & 0.515 \\
\hline X117_ttn_69 & 597.000 & 48.000 & 5.440 & 0.460 & 0.993 & 0.184 \\
\hline X117_ttn_70 & 271.500 & 6.300 & 2.408 & 0.065 & 0.807 & 0.415 \\
\hline X117_ttn_71 & 241.000 & 14.000 & 2.180 & 0.130 & 0.955 & 0.459 \\
\hline X117_ttn_72 & 375.000 & 13.000 & 3.320 & 0.120 & 0.969 & 0.301 \\
\hline X117_ttn_73 & 422.000 & 16.000 & 3.770 & 0.150 & 0.933 & 0.265 \\
\hline X117_ttn_74 & 1200.000 & 130.000 & 10.960 & 0.750 & 0.344 & 0.091 \\
\hline X117_ttn_75 & 284.000 & 14.000 & 2.570 & 0.180 & 0.877 & 0.389 \\
\hline X117_ttn_76 & 192.000 & 11.000 & 1.770 & 0.140 & 0.376 & 0.565 \\
\hline X117_ttn_77 & 404.000 & 19.000 & 3.660 & 0.160 & 0.952 & 0.273 \\
\hline X117_ttn_78 & 156.300 & 6.500 & 1.420 & 0.065 & 0.933 & 0.704 \\
\hline X117_ttn_79 & 615.000 & 29.000 & 5.380 & 0.260 & 0.935 & 0.186 \\
\hline X117_ttn_80 & 1350.000 & 140.000 & 11.900 & 1.200 & 0.966 & 0.084 \\
\hline X117_ttn_81 & 709.000 & 34.000 & 6.220 & 0.260 & 0.501 & 0.161 \\
\hline X117_ttn_82 & 452.000 & 15.000 & 4.000 & 0.130 & 0.968 & 0.250 \\
\hline X117_ttn_83 & 1103.000 & 62.000 & 9.830 & 0.580 & 0.993 & 0.102 \\
\hline X117_ttn_84 & 196.000 & 7.600 & 1.757 & 0.078 & 0.985 & 0.569 \\
\hline X117_ttn_85 & 421.000 & 16.000 & 3.800 & 0.140 & 0.812 & 0.263 \\
\hline X117_ttn_86 & 101.600 & 5.100 & 0.928 & 0.048 & 0.632 & 1.078 \\
\hline X117_ttn_87 & 118.800 & 6.700 & 1.097 & 0.061 & 0.861 & 0.912 \\
\hline X117_ttn_88 & 206.000 & 11.000 & 1.870 & 0.110 & 0.499 & 0.535 \\
\hline X117_ttn_89 & 218.000 & 16.000 & 1.920 & 0.130 & 0.947 & 0.521 \\
\hline X117_ttn_90 & 111.500 & 4.500 & 1.021 & 0.037 & 0.858 & 0.979 \\
\hline X117_ttn_91 & 581.000 & 43.000 & 4.980 & 0.400 & 0.976 & 0.201 \\
\hline X117_ttn_92 & 311.000 & 13.000 & 2.710 & 0.110 & 0.876 & 0.369 \\
\hline X117_ttn_93 & 226.600 & 6.300 & 2.043 & 0.061 & 0.861 & 0.489 \\
\hline X117_ttn_94 & 23.570 & 0.820 & 0.258 & 0.009 & 0.990 & 3.880 \\
\hline X117_ttn_95 & 103.200 & 8.100 & 0.951 & 0.071 & 0.987 & 1.052 \\
\hline X117_ttn_96 & 163.000 & 12.000 & 1.510 & 0.110 & 0.979 & 0.662 \\
\hline X117_ttn_97 & 198.600 & 7.800 & 1.816 & 0.073 & 0.978 & 0.551 \\
\hline X117_ttn_98 & 135.100 & 7.000 & 1.238 & 0.061 & 0.966 & 0.808 \\
\hline X117_ttn_99 & 192.500 & 6.000 & 1.729 & 0.050 & 0.789 & 0.578 \\
\hline X117_ttn_100 & 52.200 & 1.400 & 0.501 & 0.011 & 0.805 & 1.996 \\
\hline X117_ttn_101 & 661.000 & 17.000 & 5.830 & 0.180 & 0.205 & 0.172 \\
\hline X117_ttn_102 & 182.600 & 5.100 & 1.618 & 0.055 & 0.505 & 0.618 \\
\hline X117_ttn_103 & 689.000 & 30.000 & 6.010 & 0.260 & 0.965 & 0.166 \\
\hline X117_ttn_104 & 163.500 & 9.900 & 1.484 & 0.092 & 0.708 & 0.674 \\
\hline X117_ttn_105 & 155.000 & 13.000 & 1.390 & 0.110 & 0.605 & 0.719 \\
\hline X117_ttn_106 & 222.400 & 7.500 & 2.044 & 0.088 & 0.655 & 0.489 \\
\hline X117_ttn_107 & 279.000 & 19.000 & 2.480 & 0.170 & 0.977 & 0.403 \\
\hline X117_ttn_108 & 176.700 & 9.100 & 1.509 & 0.064 & 0.139 & 0.663 \\
\hline X117_ttn_109 & 529.000 & 52.000 & 4.520 & 0.400 & 0.965 & 0.221 \\
\hline X117_ttn_110 & 396.000 & 15.000 & 3.530 & 0.150 & 0.986 & 0.283 \\
\hline $\mathrm{x} 117 \mathrm{ttn} 111$ & 161.900 & 5.900 & 1.466 & 0.058 & 0.160 & 0.682 \\
\hline
\end{tabular}




\begin{tabular}{l|cccccc} 
X117_ttn_112 & 391.000 & 14.000 & 3.440 & 0.110 & 0.717 & 0.291 \\
X117_ttn_113 & 256.000 & 11.000 & 2.248 & 0.085 & 0.964 & 0.445 \\
X117_ttn_114 & 313.000 & 13.000 & 2.760 & 0.110 & 0.715 & 0.362 \\
X117_ttn_115 & 192.100 & 8.400 & 1.732 & 0.096 & 0.909 & 0.577 \\
X117_ttn_116 & 78.000 & 4.300 & 0.710 & 0.033 & 0.081 & 1.408
\end{tabular}




\begin{tabular}{|c|c|c|c|c|c|}
\hline Error $2 \sigma$ & ${ }^{207} \mathrm{~Pb} /{ }^{206} \mathrm{~Pb}$ & Error $2 \sigma$ & Correlation_38_6v & FinalAge ${ }^{206} \mathrm{~Pb} /{ }^{238} \mathrm{U}$ & Error $2 \sigma$ \\
\hline 0.081 & 0.100 & 0.004 & 0.061 & 1547.904 & 34.220 \\
\hline 0.072 & 0.096 & 0.002 & 0.030 & 1497.512 & 28.060 \\
\hline 0.084 & 0.101 & 0.013 & 0.001 & 1539.279 & 34.815 \\
\hline 0.076 & 0.095 & 0.001 & 0.195 & 1507.214 & 30.327 \\
\hline 0.080 & 0.094 & 0.002 & 0.666 & 1533.692 & 33.118 \\
\hline 0.072 & 0.096 & 0.002 & 0.430 & 1478.064 & 27.531 \\
\hline 0.071 & 0.096 & 0.002 & 0.134 & 1504.662 & 28.044 \\
\hline 0.089 & 0.096 & 0.002 & -0.290 & 1523.521 & 36.002 \\
\hline 0.065 & 0.095 & 0.002 & 0.000 & 1492.911 & 25.206 \\
\hline 0.078 & 0.098 & 0.003 & -0.198 & 1516.391 & 31.449 \\
\hline 0.070 & 0.095 & 0.002 & 0.009 & 1488.819 & 26.934 \\
\hline 0.084 & 0.096 & 0.003 & -0.012 & 1549.932 & 35.355 \\
\hline 0.071 & 0.096 & 0.001 & 0.096 & 1556.518 & 30.207 \\
\hline 0.073 & 0.097 & 0.003 & 0.201 & 1551.959 & 30.788 \\
\hline 0.082 & 0.095 & 0.002 & -0.254 & 1596.900 & 36.926 \\
\hline 0.075 & 0.095 & 0.002 & 0.094 & 1534.708 & 30.831 \\
\hline 0.083 & 0.096 & 0.002 & -0.201 & 1561.074 & 35.324 \\
\hline 0.097 & 0.095 & 0.002 & 0.454 & 1515.372 & 38.885 \\
\hline 0.088 & 0.098 & 0.003 & -0.579 & 1515.372 & 35.454 \\
\hline 0.086 & 0.099 & 0.004 & 0.011 & 1532.167 & 35.406 \\
\hline 0.085 & 0.095 & 0.003 & -0.246 & 1473.449 & 32.131 \\
\hline 0.071 & 0.094 & 0.002 & 0.128 & 1586.828 & 31.270 \\
\hline 0.091 & 0.096 & 0.002 & 0.650 & 1560.062 & 38.745 \\
\hline 0.100 & 0.104 & 0.009 & 0.147 & 1535.724 & 41.105 \\
\hline 0.097 & 0.097 & 0.004 & -0.249 & 1512.314 & 38.895 \\
\hline 0.095 & 0.123 & 0.028 & -0.302 & 1548.918 & 39.921 \\
\hline 0.081 & 0.101 & 0.004 & -0.350 & 1482.675 & 30.961 \\
\hline 0.080 & 0.098 & 0.003 & 0.196 & 1529.117 & 32.559 \\
\hline 0.077 & 0.095 & 0.002 & 0.550 & 1574.721 & 33.577 \\
\hline 0.099 & 0.095 & 0.002 & -0.059 & 1483.700 & 37.838 \\
\hline 0.098 & 0.102 & 0.005 & -0.192 & 1617.498 & 45.372 \\
\hline 0.100 & 0.096 & 0.002 & 0.358 & 1578.759 & 43.807 \\
\hline 0.074 & 0.096 & 0.002 & 0.198 & 1502.109 & 29.195 \\
\hline 0.151 & 0.100 & 0.007 & 0.248 & 1601.427 & 68.146 \\
\hline 0.096 & 0.101 & 0.006 & -0.073 & 1562.086 & 41.018 \\
\hline 0.076 & 0.096 & 0.004 & -0.102 & 1549.425 & 31.935 \\
\hline 0.022 & 0.840 & 0.017 & 0.008 & 10003.602 & 806.742 \\
\hline 0.030 & 0.829 & 0.010 & 0.007 & 8740.275 & 758.704 \\
\hline 0.103 & 0.811 & 0.023 & -0.116 & 5425.091 & 739.785 \\
\hline 0.012 & 0.846 & 0.014 & -0.294 & 11834.175 & 718.584 \\
\hline 0.111 & 0.809 & 0.017 & -0.051 & 4265.312 & 445.630 \\
\hline 0.056 & 0.820 & 0.010 & -0.131 & 7334.943 & 864.970 \\
\hline 0.196 & 0.722 & 0.012 & 0.020 & 2214.922 & 178.274 \\
\hline 0.008 & 0.846 & 0.011 & 0.142 & 16484.946 & 1523.819 \\
\hline 0.010 & 0.836 & 0.017 & 0.167 & 13099.680 & 889.119 \\
\hline 0.024 & 0.849 & 0.016 & 0.484 & 7754.858 & 432.674 \\
\hline 0.036 & 0.807 & 0.019 & -0.111 & 6147.214 & 354.573 \\
\hline 0.008 & 0.846 & 0.012 & -0.153 & 17584.869 & 2090.509 \\
\hline
\end{tabular}




\begin{tabular}{|c|c|c|c|c|c|}
\hline 0.102 & 0.806 & 0.016 & 0.171 & 6233.578 & 1032.556 \\
\hline 0.018 & 0.829 & 0.012 & 0.329 & 8167.269 & 384.342 \\
\hline 0.003 & 0.836 & 0.013 & 0.277 & 16374.059 & 574.774 \\
\hline 0.003 & 0.841 & 0.011 & 0.219 & 16994.012 & 655.633 \\
\hline 0.006 & 0.842 & 0.011 & 0.340 & 17668.590 & 1584.080 \\
\hline 0.006 & 0.850 & 0.011 & -0.149 & 19148.522 & 2277.122 \\
\hline 0.004 & 0.851 & 0.017 & 0.353 & 16998.628 & 838.791 \\
\hline 0.001 & 0.843 & 0.009 & 0.027 & 28150.930 & 1592.083 \\
\hline 0.019 & 0.833 & 0.009 & 0.052 & 9048.464 & 530.529 \\
\hline 0.066 & 0.804 & 0.015 & -0.001 & 5341.188 & 455.450 \\
\hline 0.088 & 0.826 & 0.017 & 0.285 & 5723.715 & 720.468 \\
\hline 0.135 & 0.765 & 0.022 & 0.073 & 2618.092 & 177.675 \\
\hline 0.068 & 0.744 & 0.016 & 0.302 & 2413.011 & 74.410 \\
\hline 0.031 & 0.808 & 0.010 & 0.450 & 4545.210 & 146.477 \\
\hline 0.038 & 0.820 & 0.017 & 0.300 & 4432.761 & 165.836 \\
\hline 0.006 & 0.848 & 0.018 & 0.070 & 24155.670 & 5542.968 \\
\hline 0.003 & 0.848 & 0.011 & 0.107 & 17832.839 & 777.943 \\
\hline 0.005 & 0.833 & 0.010 & -0.256 & 18264.067 & 1369.805 \\
\hline 0.002 & 0.842 & 0.011 & 0.033 & 24874.277 & 2412.376 \\
\hline 0.114 & 0.803 & 0.023 & -0.078 & 5141.062 & 716.377 \\
\hline 0.007 & 0.844 & 0.010 & 0.306 & 15434.342 & 1053.750 \\
\hline 0.000 & 0.859 & 0.019 & 0.341 & \#VALUE! & \#VALUE! \\
\hline 0.009 & 0.844 & 0.017 & -0.148 & 14512.759 & 1143.947 \\
\hline 0.005 & 0.847 & 0.007 & -0.253 & 22882.304 & 3655.753 \\
\hline 1.005 & 0.480 & 0.060 & -0.706 & 719.042 & 85.310 \\
\hline 0.047 & 0.805 & 0.017 & -0.027 & 5327.098 & 323.357 \\
\hline 0.411 & 0.294 & 0.012 & 0.400 & 465.009 & 14.298 \\
\hline 0.046 & 0.798 & 0.014 & 0.343 & 5483.179 & 335.288 \\
\hline 0.039 & 0.826 & 0.012 & 0.182 & 8221.517 & 828.525 \\
\hline 0.023 & 0.821 & 0.010 & -0.117 & 6929.911 & 308.794 \\
\hline 0.020 & 0.834 & 0.012 & 0.092 & 14778.629 & 2656.832 \\
\hline 0.035 & 0.822 & 0.011 & 0.085 & 7617.903 & 606.736 \\
\hline 0.096 & 0.771 & 0.008 & -0.245 & 3363.517 & 220.961 \\
\hline 0.050 & 0.807 & 0.016 & -0.193 & 7983.073 & 977.519 \\
\hline 0.002 & 0.844 & 0.019 & 0.104 & 30359.582 & 6347.913 \\
\hline 0.002 & 0.839 & 0.032 & 0.419 & 27797.844 & 3663.575 \\
\hline 0.069 & 0.832 & 0.032 & -0.025 & 9095.806 & 1936.527 \\
\hline 0.034 & 0.826 & 0.017 & -0.054 & 7578.233 & 575.134 \\
\hline 0.059 & 0.812 & 0.011 & 0.120 & 6402.912 & 640.291 \\
\hline 0.010 & 0.840 & 0.011 & 0.032 & 10336.308 & 390.541 \\
\hline 0.016 & 0.822 & 0.022 & 0.066 & 12125.644 & 1068.627 \\
\hline 0.001 & 0.834 & 0.034 & -0.180 & 38377.072 & 7495.522 \\
\hline 0.721 & 0.306 & 0.018 & -0.694 & 495.526 & 28.528 \\
\hline 0.383 & 0.217 & 0.007 & 0.407 & 415.639 & 10.609 \\
\hline 0.199 & 0.479 & 0.009 & 0.001 & 709.810 & 16.465 \\
\hline 0.238 & 0.708 & 0.013 & -0.387 & 1872.221 & 150.000 \\
\hline 0.386 & 0.410 & 0.015 & -0.474 & 631.966 & 25.156 \\
\hline 0.543 & 0.407 & 0.023 & -0.636 & 573.255 & 28.971 \\
\hline 0.770 & 0.479 & 0.046 & -0.309 & 675.651 & 57.476 \\
\hline
\end{tabular}




\begin{tabular}{|c|c|c|c|c|c|}
\hline 0.240 & 0.602 & 0.014 & -0.463 & 1087.701 & 47.935 \\
\hline 0.076 & 0.808 & 0.024 & 0.070 & 5082.723 & 465.916 \\
\hline 0.349 & 0.423 & 0.008 & 0.126 & 655.302 & 24.497 \\
\hline 0.505 & 0.282 & 0.010 & -0.149 & 454.805 & 16.799 \\
\hline 0.234 & 0.455 & 0.014 & 0.201 & 702.299 & 18.915 \\
\hline 0.781 & 0.369 & 0.018 & -0.599 & 533.024 & 35.865 \\
\hline 0.079 & 0.783 & 0.016 & -0.216 & 3465.994 & 194.719 \\
\hline 0.064 & 0.798 & 0.017 & -0.107 & 4458.637 & 286.211 \\
\hline 0.067 & 0.823 & 0.016 & 0.351 & 5480.425 & 490.784 \\
\hline 0.416 & 0.645 & 0.022 & 0.680 & 1218.154 & 105.417 \\
\hline 0.222 & 0.632 & 0.015 & 0.240 & 1194.631 & 53.981 \\
\hline 0.150 & 0.748 & 0.013 & -0.237 & 2837.638 & 236.042 \\
\hline 0.021 & 0.819 & 0.010 & -0.010 & 6069.741 & 201.807 \\
\hline 0.011 & 0.838 & 0.012 & 0.052 & 10058.003 & 428.000 \\
\hline 0.013 & 0.829 & 0.011 & 0.315 & 7094.990 & 180.381 \\
\hline 0.037 & 0.819 & 0.014 & 0.246 & 5924.814 & 326.317 \\
\hline 0.015 & 0.833 & 0.014 & -0.048 & 6639.655 & 176.959 \\
\hline 0.036 & 0.828 & 0.012 & 0.341 & 8920.491 & 954.701 \\
\hline 0.023 & 0.828 & 0.011 & -0.004 & 7103.562 & 328.672 \\
\hline 0.012 & 0.839 & 0.011 & 0.121 & 9016.708 & 325.193 \\
\hline 0.027 & 0.832 & 0.030 & -0.682 & 6504.768 & 306.019 \\
\hline 0.021 & 0.833 & 0.013 & 0.274 & 7457.735 & 342.098 \\
\hline 0.124 & 0.812 & 0.019 & 0.457 & 5452.817 & 901.970 \\
\hline 0.109 & 0.788 & 0.037 & 0.002 & 3305.873 & 241.773 \\
\hline 0.023 & 0.835 & 0.013 & 0.108 & 6648.854 & 272.585 \\
\hline 0.009 & 0.828 & 0.018 & 0.126 & 14836.968 & 1188.278 \\
\hline 0.046 & 0.797 & 0.020 & 0.021 & 3336.681 & 103.349 \\
\hline 0.009 & 0.843 & 0.014 & 0.075 & 10218.503 & 368.709 \\
\hline 0.034 & 0.825 & 0.027 & -0.014 & 4733.532 & 174.669 \\
\hline 0.077 & 0.800 & 0.015 & -0.222 & 6951.875 & 1039.198 \\
\hline 0.041 & 0.808 & 0.017 & 0.294 & 3990.087 & 139.676 \\
\hline 0.026 & 0.829 & 0.015 & 0.043 & 8756.868 & 666.613 \\
\hline 0.046 & 0.816 & 0.016 & 0.367 & 6009.116 & 429.223 \\
\hline 0.035 & 0.829 & 0.034 & 0.199 & 10895.058 & 1676.163 \\
\hline 0.009 & 0.821 & 0.012 & -0.221 & 13191.953 & 782.905 \\
\hline 0.014 & 0.829 & 0.017 & 0.673 & 9235.782 & 405.332 \\
\hline 0.198 & 0.718 & 0.016 & -0.060 & 1656.503 & 96.111 \\
\hline 0.119 & 0.828 & 0.025 & -0.094 & 6134.781 & 1157.506 \\
\hline 0.253 & 0.713 & 0.022 & -0.402 & 1852.906 & 155.800 \\
\hline 0.173 & 0.737 & 0.019 & 0.377 & 2178.242 & 151.718 \\
\hline 0.132 & 0.817 & 0.015 & -0.024 & 5082.723 & 804.764 \\
\hline 0.044 & 0.811 & 0.029 & 0.094 & 5906.790 & 393.786 \\
\hline 0.142 & 0.708 & 0.033 & 0.101 & 2136.726 & 119.613 \\
\hline 0.142 & 0.794 & 0.019 & 0.232 & 6134.781 & 1389.007 \\
\hline 0.227 & 0.758 & 0.043 & 0.065 & 2417.443 & 249.714 \\
\hline 0.150 & 0.736 & 0.039 & -0.049 & 2791.815 & 226.642 \\
\hline 0.129 & 0.768 & 0.035 & 0.207 & 2960.978 & 223.470 \\
\hline 0.097 & 0.812 & 0.024 & 0.210 & 4813.460 & 520.374 \\
\hline 0.208 & 0.776 & 0.027 & -0.419 & 4403.525 & 898.679 \\
\hline
\end{tabular}




\begin{tabular}{|c|c|c|c|c|c|}
\hline 0.061 & 0.792 & 0.022 & 0.271 & 4007.421 & 209.204 \\
\hline 0.028 & 0.828 & 0.016 & 0.192 & 11047.851 & 1384.017 \\
\hline 1.006 & 0.352 & 0.048 & 0.220 & 505.666 & 41.519 \\
\hline 0.204 & 0.761 & 0.046 & -0.002 & 2260.479 & 193.755 \\
\hline 0.252 & 0.741 & 0.038 & 0.215 & 1991.647 & 181.559 \\
\hline 0.270 & 0.771 & 0.030 & 0.044 & 2770.878 & 402.474 \\
\hline 0.100 & 0.783 & 0.029 & -0.010 & 3625.907 & 273.744 \\
\hline 0.142 & 0.805 & 0.041 & -0.069 & 4069.439 & 508.680 \\
\hline 0.118 & 0.728 & 0.031 & -0.078 & 2359.588 & 122.784 \\
\hline 0.084 & 0.795 & 0.038 & 0.791 & 5697.132 & 682.051 \\
\hline 0.043 & 0.784 & 0.035 & 0.090 & 5860.207 & 375.654 \\
\hline 0.041 & 0.811 & 0.033 & -0.064 & 12164.833 & 2823.979 \\
\hline 0.403 & 0.658 & 0.041 & -0.164 & 1621.509 & 187.097 \\
\hline 0.062 & 0.808 & 0.032 & 0.367 & 8167.269 & 1281.140 \\
\hline 0.046 & 0.850 & 0.030 & 0.108 & 8094.221 & 935.189 \\
\hline 0.042 & 0.842 & 0.040 & -0.074 & 5554.381 & 316.929 \\
\hline 0.095 & 0.817 & 0.022 & 0.321 & 4452.178 & 420.608 \\
\hline 0.021 & 0.839 & 0.034 & 0.282 & 13795.753 & 2207.320 \\
\hline 0.233 & 0.690 & 0.040 & -0.064 & 1365.869 & 75.239 \\
\hline 0.028 & 0.837 & 0.046 & -0.215 & 9795.411 & 987.773 \\
\hline 0.156 & 0.786 & 0.017 & -0.011 & 3789.116 & 473.640 \\
\hline 0.156 & 0.777 & 0.027 & 0.323 & 5198.877 & 1006.234 \\
\hline 0.023 & 0.822 & 0.021 & -0.049 & 14164.220 & 2655.791 \\
\hline 0.032 & 0.820 & 0.033 & 0.011 & 5657.050 & 253.661 \\
\hline 0.313 & 0.596 & 0.017 & -0.058 & 961.221 & 48.420 \\
\hline 0.121 & 0.767 & 0.036 & 0.090 & 3870.965 & 385.685 \\
\hline 0.267 & 0.777 & 0.034 & 0.464 & 3607.515 & 721.503 \\
\hline 0.158 & 0.806 & 0.033 & -0.114 & 3702.586 & 453.280 \\
\hline 0.524 & 0.235 & 0.021 & -0.324 & 404.751 & 13.742 \\
\hline 0.675 & 0.543 & 0.045 & -0.185 & 867.242 & 84.315 \\
\hline 0.034 & 0.842 & 0.028 & 0.502 & 6660.335 & 404.772 \\
\hline 0.136 & 0.798 & 0.045 & 0.380 & 4271.961 & 545.357 \\
\hline 0.412 & 0.805 & 0.027 & 0.037 & 5480.425 & 3026.503 \\
\hline 0.033 & 0.790 & 0.030 & -0.011 & 5705.118 & 268.618 \\
\hline 0.094 & 0.798 & 0.019 & 0.022 & 5802.813 & 794.906 \\
\hline 0.051 & 0.797 & 0.024 & -0.171 & 5070.991 & 309.517 \\
\hline 0.108 & 0.827 & 0.049 & -0.137 & 6258.043 & 1106.605 \\
\hline 0.076 & 0.805 & 0.037 & 0.061 & 10111.948 & 2927.143 \\
\hline 0.067 & 0.801 & 0.019 & 0.023 & 6354.983 & 718.718 \\
\hline 0.128 & 0.775 & 0.013 & -0.486 & 3774.775 & 384.117 \\
\hline 0.015 & 0.801 & 0.011 & -0.154 & 7349.390 & 228.049 \\
\hline 0.014 & 0.820 & 0.010 & 0.087 & 10464.727 & 591.373 \\
\hline 0.012 & 0.814 & 0.013 & 0.620 & 8690.238 & 304.921 \\
\hline 0.021 & 0.837 & 0.017 & -0.013 & 10738.554 & 976.232 \\
\hline 0.009 & 0.828 & 0.011 & -0.006 & 12203.784 & 649.137 \\
\hline 0.007 & 0.824 & 0.008 & 0.224 & 12902.369 & 604.799 \\
\hline 0.026 & 0.799 & 0.017 & -0.239 & 5181.587 & 167.961 \\
\hline 0.012 & 0.796 & 0.008 & 0.661 & 7764.530 & 226.119 \\
\hline 0.008 & 0.810 & 0.012 & 0.566 & 9574.436 & 255.057 \\
\hline
\end{tabular}




\begin{tabular}{|c|c|c|c|c|c|}
\hline 0.021 & 0.805 & 0.012 & -0.107 & 8203.485 & 446.882 \\
\hline 0.011 & 0.814 & 0.009 & 0.203 & 14465.084 & 1355.565 \\
\hline 0.028 & 0.791 & 0.013 & -0.172 & 6683.235 & 337.834 \\
\hline 0.027 & 0.815 & 0.013 & 0.025 & 9506.933 & 874.525 \\
\hline 0.008 & 0.813 & 0.009 & 0.709 & 12928.450 & 663.513 \\
\hline 0.031 & 0.823 & 0.013 & 0.227 & 12544.143 & 2299.760 \\
\hline 0.023 & 0.813 & 0.012 & 0.152 & 7983.073 & 456.176 \\
\hline 0.022 & 0.811 & 0.009 & 0.445 & 7793.459 & 397.964 \\
\hline 0.012 & 0.839 & 0.016 & -0.295 & 11059.456 & 582.077 \\
\hline 0.015 & 0.800 & 0.011 & 0.311 & 8468.807 & 342.489 \\
\hline 0.004 & 0.813 & 0.009 & 0.133 & 15783.501 & 627.159 \\
\hline 0.019 & 0.831 & 0.010 & 0.058 & 9781.290 & 659.413 \\
\hline 0.022 & 0.795 & 0.011 & -0.244 & 7192.890 & 329.499 \\
\hline 0.021 & 0.805 & 0.013 & -0.206 & 7850.931 & 395.845 \\
\hline 0.031 & 0.806 & 0.009 & -0.157 & 7754.858 & 565.805 \\
\hline 0.042 & 0.808 & 0.010 & -0.099 & 8075.829 & 839.886 \\
\hline 0.042 & 0.782 & 0.024 & 0.314 & 5088.580 & 254.006 \\
\hline 0.005 & 0.827 & 0.006 & 0.157 & 13116.555 & 473.379 \\
\hline 0.009 & 0.825 & 0.012 & 0.148 & 15733.160 & 1426.193 \\
\hline 0.023 & 0.828 & 0.013 & 0.156 & 9638.348 & 779.982 \\
\hline 0.074 & 0.759 & 0.022 & 0.460 & 3005.619 & 131.559 \\
\hline 0.019 & 0.814 & 0.014 & 0.541 & 8257.430 & 412.872 \\
\hline 0.019 & 0.802 & 0.011 & 0.309 & 7131.344 & 278.485 \\
\hline 0.016 & 0.822 & 0.008 & 0.080 & 8673.472 & 397.025 \\
\hline 0.016 & 0.835 & 0.011 & 0.097 & 14092.192 & 1783.822 \\
\hline 0.019 & 0.825 & 0.017 & 0.672 & 8399.115 & 438.760 \\
\hline 0.035 & 0.818 & 0.012 & -0.030 & 8806.393 & 904.766 \\
\hline 0.023 & 0.834 & 0.013 & -0.012 & 11678.080 & 1345.716 \\
\hline 0.018 & 0.813 & 0.012 & -0.167 & 8706.960 & 456.659 \\
\hline 0.052 & 0.798 & 0.021 & 0.125 & 7578.233 & 879.616 \\
\hline 0.028 & 0.834 & 0.012 & -0.082 & 9989.930 & 1050.154 \\
\hline 0.009 & 0.816 & 0.017 & 0.299 & 13175.274 & 784.242 \\
\hline 0.020 & 0.809 & 0.010 & 0.079 & 11365.138 & 1082.394 \\
\hline 0.041 & 0.819 & 0.015 & -0.009 & 9095.806 & 1144.311 \\
\hline 0.035 & 0.795 & 0.020 & -0.100 & 7518.266 & 578.328 \\
\hline 0.019 & 0.823 & 0.010 & 0.051 & 9989.930 & 700.103 \\
\hline 0.032 & 0.807 & 0.011 & 0.224 & 8328.662 & 694.055 \\
\hline 0.046 & 0.786 & 0.010 & 0.224 & 5416.749 & 329.036 \\
\hline 0.026 & 0.786 & 0.010 & -0.186 & 5201.755 & 167.663 \\
\hline 0.026 & 0.822 & 0.012 & 0.219 & 7696.519 & 468.484 \\
\hline 0.103 & 0.785 & 0.012 & -0.209 & 3976.187 & 349.606 \\
\hline 0.015 & 0.827 & 0.014 & -0.038 & 8673.472 & 366.485 \\
\hline 0.028 & 0.799 & 0.013 & 0.821 & 5103.201 & 173.348 \\
\hline 0.034 & 0.773 & 0.013 & 0.136 & 3600.144 & 91.447 \\
\hline 0.022 & 0.805 & 0.011 & 0.050 & 6816.716 & 275.716 \\
\hline 0.023 & 0.811 & 0.006 & 0.195 & 8888.097 & 598.525 \\
\hline 0.076 & 0.794 & 0.018 & 0.170 & 5397.244 & 535.604 \\
\hline 0.045 & 0.789 & 0.014 & 0.397 & 4174.870 & 169.561 \\
\hline 0.050 & 0.791 & 0.023 & -0.119 & 4347.938 & 207.690 \\
\hline 0.042 & 0.795 & 0.014 & -0.411 & 4275.283 & 168.104 \\
\hline
\end{tabular}




\begin{tabular}{|c|c|c|c|c|c|}
\hline 0.013 & 0.793 & 0.010 & 0.318 & 7216.083 & 188.884 \\
\hline 0.231 & 0.600 & 0.024 & 0.026 & 1014.315 & 39.882 \\
\hline 0.080 & 0.706 & 0.016 & 0.393 & 2029.400 & 60.334 \\
\hline 0.135 & 0.773 & 0.015 & -0.161 & 3537.143 & 348.392 \\
\hline 0.029 & 0.797 & 0.013 & 0.597 & 6521.198 & 331.650 \\
\hline 0.320 & 0.734 & 0.022 & -0.106 & 2319.227 & 321.371 \\
\hline 0.032 & 0.821 & 0.023 & 0.091 & 6951.875 & 430.013 \\
\hline 0.016 & 0.809 & 0.011 & -0.463 & 12006.630 & 1015.26 \\
\hline 0.011 & 0.818 & 0.011 & 0.438 & 7904.114 & 213.359 \\
\hline 0.027 & 0.805 & 0.014 & 0.408 & 7457.735 & 444.727 \\
\hline 0.011 & 0.815 & 0.009 & 0.425 & 9432.750 & 340.943 \\
\hline 0.011 & 0.811 & 0.013 & 0.413 & 10071.531 & 400.724 \\
\hline 0.006 & 0.819 & 0.021 & -0.075 & 15997.214 & 1094.70 \\
\hline 0.027 & 0.823 & 0.013 & 0.741 & 8203.485 & 574.563 \\
\hline 0.045 & 0.801 & 0.019 & -0.310 & 6567.912 & 519.496 \\
\hline 0.012 & 0.789 & 0.011 & 0.089 & 9921.131 & 433.711 \\
\hline 0.032 & 0.803 & 0.015 & 0.472 & 5697.132 & 260.784 \\
\hline 0.009 & 0.827 & 0.013 & 0.275 & 11946.289 & 577.330 \\
\hline 0.008 & 0.816 & 0.010 & -0.042 & 16484.946 & 1662.34 \\
\hline 0.007 & 0.812 & 0.016 & 0.048 & 12743.626 & 532.692 \\
\hline 0.008 & 0.816 & 0.009 & 0.292 & 10375.103 & 337.191 \\
\hline 0.006 & 0.815 & 0.008 & 0.125 & 15357.422 & 906.135 \\
\hline 0.025 & 0.810 & 0.011 & 0.431 & 6537.587 & 290.229 \\
\hline 0.010 & 0.812 & 0.015 & 0.155 & 10111.948 & 372.545 \\
\hline 0.056 & 0.779 & 0.018 & 0.670 & 4231.963 & 218.895 \\
\hline 0.051 & 0.786 & 0.013 & -0.327 & 4773.620 & 265.443 \\
\hline 0.031 & 0.805 & 0.010 & 0.542 & 6796.532 & 399.796 \\
\hline 0.035 & 0.814 & 0.014 & -0.168 & 6907.872 & 467.720 \\
\hline 0.035 & 0.797 & 0.011 & 0.153 & 4535.648 & 164.367 \\
\hline 0.016 & 0.840 & 0.014 & 0.495 & 11528.900 & 926.016 \\
\hline 0.015 & 0.829 & 0.013 & 0.035 & 8451.454 & 343.048 \\
\hline 0.015 & 0.811 & 0.012 & 0.305 & 7173.853 & 214.197 \\
\hline 0.130 & 0.664 & 0.010 & -0.559 & 1478.064 & 49.326 \\
\hline 0.079 & 0.786 & 0.010 & -0.163 & 4308.410 & 321.658 \\
\hline 0.048 & 0.783 & 0.010 & -0.029 & 5932.524 & 432.171 \\
\hline 0.022 & 0.799 & 0.012 & 0.052 & 6674.085 & 268.286 \\
\hline 0.040 & 0.790 & 0.010 & -0.022 & 5193.119 & 255.881 \\
\hline 0.017 & 0.811 & 0.013 & 0.205 & 6471.782 & 187.154 \\
\hline 0.044 & 0.748 & 0.010 & 0.116 & 2618.092 & 57.483 \\
\hline 0.005 & 0.835 & 0.013 & 0.632 & 12385.655 & 382.404 \\
\hline 0.021 & 0.818 & 0.018 & 0.638 & 6204.098 & 210.893 \\
\hline 0.007 & 0.831 & 0.011 & 0.203 & 12553.345 & 543.073 \\
\hline 0.042 & 0.790 & 0.010 & -0.045 & 5865.400 & 363.623 \\
\hline 0.057 & 0.798 & 0.019 & 0.020 & 5616.718 & 444.488 \\
\hline 0.021 & 0.811 & 0.024 & 0.614 & 7175.971 & 308.946 \\
\hline 0.028 & 0.815 & 0.012 & 0.110 & 8038.887 & 551.053 \\
\hline 0.028 & 0.811 & 0.031 & 0.115 & 5929.955 & 251.502 \\
\hline 0.020 & 0.813 & 0.017 & 0.000 & 11012.911 & 974.594 \\
\hline 0.012 & 0.817 & 0.013 & 0.575 & 9738.739 & 413.827 \\
\hline 0.027 & 0.799 & 0.021 & -0.134 & 5818.517 & 230.2 \\
\hline
\end{tabular}




\begin{tabular}{lllllll}
0.009 & 0.820 & 0.013 & 0.045 & 9609.376 & 307.277 \\
0.017 & 0.820 & 0.014 & 0.030 & 7594.130 & 287.145 \\
0.014 & 0.819 & 0.022 & 0.198 & 8537.753 & 340.273 \\
0.032 & 0.817 & 0.026 & 0.338 & 6478.865 & 359.106 \\
\hline 0.065 & 0.785 & 0.018 & 0.130 & 3458.458 & 160.745
\end{tabular}




\begin{tabular}{|c|c|c|c|c|c|}
\hline inalAge ${ }^{207} \mathrm{~Pb} /{ }^{235} \mathrm{l}$ & Error $2 \sigma$ & alAge ${ }^{207} \mathrm{~Pb} /{ }^{201}$ & Error $2 \sigma$ & FinalAge ${ }^{207} \mathrm{~Pb}$ corr & Error $2 \sigma$ \\
\hline 1599.072 & 83.502 & 1624.083 & 60.091 & 1540.952 & 33.594 \\
\hline 1520.196 & 29.361 & 1543.853 & 30.619 & 1493.591 & 27.240 \\
\hline 1599.072 & 229.632 & 1642.570 & 211.420 & 1529.869 & 40.345 \\
\hline 1522.692 & 32.379 & 1522.114 & 17.680 & 1505.951 & 29.357 \\
\hline 1516.326 & 29.870 & 1516.130 & 30.515 & 1535.215 & 32.245 \\
\hline 1501.392 & 30.143 & 1541.890 & 24.168 & 1472.768 & 26.619 \\
\hline 1526.538 & 34.922 & 1553.632 & 35.493 & 1500.476 & 27.291 \\
\hline 1534.858 & 39.522 & 1537.955 & 30.598 & 1522.270 & 34.950 \\
\hline 1504.396 & 48.672 & 1522.114 & 36.968 & 1490.477 & 24.619 \\
\hline 1545.107 & 47.475 & 1582.592 & 43.691 & 1510.617 & 30.640 \\
\hline 1501.855 & 41.657 & 1535.984 & 32.201 & 1484.887 & 26.165 \\
\hline 1550.635 & 38.282 & 1555.580 & 43.569 & 1549.426 & 34.550 \\
\hline 1553.937 & 35.200 & 1539.924 & 22.551 & 1558.009 & 29.394 \\
\hline 1579.974 & 46.470 & 1565.283 & 51.691 & 1550.758 & 30.316 \\
\hline 1567.039 & 46.841 & 1530.055 & 30.569 & 1603.150 & 36.149 \\
\hline 1542.888 & 56.184 & 1528.074 & 32.170 & 1535.288 & 30.050 \\
\hline 1560.509 & 47.029 & 1549.728 & 29.027 & 1562.101 & 34.394 \\
\hline 1525.182 & 33.204 & 1526.090 & 28.946 & 1514.456 & 37.706 \\
\hline 1542.888 & 69.149 & 1584.503 & 50.173 & 1509.345 & 34.549 \\
\hline 1542.888 & 64.827 & 1607.248 & 58.386 & 1525.450 & 34.665 \\
\hline 1497.449 & 62.209 & 1534.010 & 41.851 & 1468.489 & 31.216 \\
\hline 1558.542 & 39.381 & 1510.122 & 27.282 & 1593.877 & 30.629 \\
\hline 1560.509 & 51.304 & 1547.772 & 33.858 & 1561.173 & 37.747 \\
\hline 1609.530 & 112.003 & 1693.150 & 140.280 & 1521.170 & 42.099 \\
\hline 1560.509 & 102.609 & 1571.075 & 63.037 & 1507.230 & 38.059 \\
\hline 1784.899 & 446.225 & 2000.193 & 455.328 & 1501.864 & 61.561 \\
\hline 1571.369 & 110.421 & 1640.731 & 56.913 & 1469.121 & 30.187 \\
\hline 1547.322 & 43.101 & 1580.679 & 51.772 & 1524.559 & 31.884 \\
\hline 1567.473 & 37.888 & 1534.010 & 25.755 & 1578.443 & 32.758 \\
\hline 1495.123 & 48.947 & 1524.103 & 25.723 & 1480.364 & 36.563 \\
\hline 1642.287 & 117.887 & 1660.831 & 83.042 & 1613.173 & 44.936 \\
\hline 1567.039 & 42.583 & 1545.814 & 33.850 & 1581.796 & 42.728 \\
\hline 1515.413 & 41.753 & 1541.890 & 32.223 & 1498.738 & 28.355 \\
\hline 1650.314 & 133.481 & 1625.942 & 113.702 & 1599.049 & 67.252 \\
\hline 1632.163 & 134.991 & 1649.901 & 92.746 & 1553.855 & 40.778 \\
\hline 1547.322 & 64.651 & 1545.814 & 59.640 & 1549.748 & 31.601 \\
\hline 6157.065 & 516.677 & 4991.118 & 101.011 & -401.040 & 610.286 \\
\hline 5888.300 & 501.132 & 4972.394 & 59.981 & 154.398 & 300.827 \\
\hline 5121.008 & 831.333 & 4941.178 & 140.132 & 748.550 & 250.532 \\
\hline 6498.713 & 410.900 & 5001.222 & 82.763 & -9194.813 & 3356.044 \\
\hline 4725.552 & 499.818 & 4937.664 & 103.758 & 384.788 & 139.866 \\
\hline 5556.451 & 656.458 & 4957.224 & 59.835 & 2392.282 & 349.571 \\
\hline 3792.746 & 324.563 & 4775.137 & 79.365 & 454.768 & 55.969 \\
\hline 7326.979 & 646.498 & 5001.222 & 65.028 & -1074.208 & 1543.692 \\
\hline 6734.022 & 453.081 & 4984.340 & 101.356 & -343.904 & 1074.347 \\
\hline 5680.775 & 296.757 & 5006.245 & 94.346 & -141.388 & 347.474 \\
\hline 5284.060 & 379.518 & 4934.141 & 116.169 & 1385.289 & 229.824 \\
\hline 7553.406 & 933.068 & 5001.222 & 70.939 & 0.000 & 2202.335 \\
\hline
\end{tabular}




\begin{tabular}{|c|c|c|c|c|c|}
\hline 5284.060 & 846.617 & 4932.376 & 97.913 & 1511.353 & 307.697 \\
\hline 5753.594 & 239.733 & 4972.394 & 71.977 & 0.000 & 317.777 \\
\hline 7326.232 & 253.372 & 4984.340 & 77.508 & 0.000 & 1650.374 \\
\hline 7424.365 & 267.813 & 4992.807 & 65.304 & -588.624 & 1570.171 \\
\hline 7529.243 & 589.640 & 4994.495 & 65.249 & -790.532 & 1805.057 \\
\hline 7777.472 & 917.155 & 5007.915 & 64.808 & 0.000 & 3649.522 \\
\hline 7439.169 & 288.947 & 5009.583 & 100.074 & -1819.880 & 2556.074 \\
\hline 9246.283 & 523.374 & 4996.011 & 53.345 & 0.000 & 14678.327 \\
\hline 5948.046 & 340.862 & 4979.404 & 55.586 & 62.943 & 310.882 \\
\hline 5039.188 & 461.334 & 4928.839 & 91.956 & 869.468 & 169.742 \\
\hline 5244.236 & 723.343 & 4967.242 & 102.231 & 464.039 & 210.263 \\
\hline 4035.192 & 286.019 & 4857.929 & 139.705 & 371.889 & 92.303 \\
\hline 3920.120 & 126.455 & 4818.134 & 103.616 & 420.276 & 61.774 \\
\hline 4814.386 & 144.093 & 4935.904 & 61.088 & 470.880 & 99.522 \\
\hline 4792.895 & 168.096 & 4956.877 & 102.765 & 264.286 & 145.243 \\
\hline 8507.043 & 1760.078 & 5004.573 & 106.229 & \#VALUE! & \#VALUE! \\
\hline 7586.873 & 310.902 & 5004.573 & 64.918 & 0.000 & 1908.918 \\
\hline 7639.215 & 619.396 & 4978.380 & 58.006 & 0.000 & 1664.220 \\
\hline 8724.665 & 825.525 & 4994.495 & 65.249 & 0.000 & 8419.178 \\
\hline 5010.381 & 689.835 & 4927.067 & 141.124 & 785.949 & 236.327 \\
\hline 7164.721 & 469.818 & 4997.526 & 56.857 & 0.000 & 1993.199 \\
\hline \#VALUE! & \#VALUE! & 5022.854 & 111.099 & 0.000 & \#\#\#\#\#\#\#\#\#\# \\
\hline 7065.491 & 645.373 & 4997.862 & 100.668 & \#VALUE! & \#VALUE! \\
\hline 8441.994 & 1386.308 & 5002.898 & 40.756 & 0.000 & 10115.893 \\
\hline 2231.025 & 474.093 & 4180.933 & 522.617 & 348.264 & 67.881 \\
\hline 5052.591 & 330.051 & 4930.609 & 104.125 & 839.021 & 177.243 \\
\hline 1420.233 & 74.504 & 3438.894 & 140.363 & 329.210 & 12.206 \\
\hline 5101.163 & 337.825 & 4918.171 & 86.284 & 1098.240 & 160.127 \\
\hline 5774.458 & 569.589 & 4967.242 & 72.163 & \#NUM! & \#VALUE! \\
\hline 5481.203 & 274.060 & 4958.610 & 60.397 & 1627.507 & 176.120 \\
\hline 7035.120 & 1310.464 & 4980.938 & 71.668 & 3385.691 & 4883.850 \\
\hline 5634.438 & 440.190 & 4960.341 & 66.379 & 2969.523 & 401.525 \\
\hline 4343.864 & 262.709 & 4868.901 & 48.632 & 524.547 & 64.606 \\
\hline 5703.174 & 749.322 & 4934.141 & 97.827 & \#VALUE! & \#VALUE! \\
\hline 9602.760 & 1875.539 & 4997.862 & 112.511 & 0.000 & 18707.297 \\
\hline 9150.644 & 1115.932 & 4989.427 & 190.300 & 0.000 & 25304.139 \\
\hline 5968.153 & 1274.100 & 4977.527 & 191.443 & \#VALUE! & \#VALUE! \\
\hline 5642.309 & 481.127 & 4967.242 & 102.231 & 2371.912 & 341.172 \\
\hline 5316.994 & 540.229 & 4942.932 & 66.961 & 1452.917 & 206.668 \\
\hline 6225.543 & 244.139 & 4991.118 & 65.360 & -448.218 & 478.129 \\
\hline 6541.646 & 563.395 & 4960.341 & 132.759 & 0.000 & 1106.807 \\
\hline 10923.414 & 2324.131 & 4980.938 & 203.060 & 0.000 & 109771.265 \\
\hline 1509.001 & 154.430 & 3500.863 & 205.933 & 344.120 & 22.446 \\
\hline 1114.158 & 24.002 & 2954.841 & 96.902 & 333.391 & 9.170 \\
\hline 2195.434 & 68.518 & 4177.848 & 80.243 & 344.383 & 12.061 \\
\hline 3580.607 & 325.510 & 4747.041 & 87.163 & 403.295 & 49.259 \\
\hline 1955.331 & 116.786 & 3946.162 & 144.372 & 360.241 & 18.627 \\
\hline 1849.336 & 174.937 & 3935.141 & 222.379 & 327.230 & 23.293 \\
\hline 2231.025 & 362.541 & 4177.848 & 401.213 & 326.647 & 47.855 \\
\hline
\end{tabular}




\begin{tabular}{|c|c|c|c|c|c|}
\hline 2852.620 & 179.203 & 4512.833 & 104.950 & 368.837 & 26.604 \\
\hline 5003.050 & 474.742 & 4935.904 & 146.611 & 657.971 & 228.086 \\
\hline 2012.875 & 77.171 & 3993.978 & 77.370 & 363.191 & 15.274 \\
\hline 1379.306 & 62.045 & 3374.039 & 119.647 & 328.581 & 13.270 \\
\hline 2147.586 & 88.378 & 4101.635 & 126.204 & 362.310 & 16.052 \\
\hline 1712.341 & 167.342 & 3787.319 & 184.747 & 328.895 & 24.929 \\
\hline 4425.029 & 269.749 & 4891.124 & 99.946 & 457.887 & 98.393 \\
\hline 4762.583 & 317.800 & 4918.171 & 104.773 & 589.581 & 142.139 \\
\hline 5053.291 & 421.108 & 4962.070 & 96.468 & 462.836 & 185.064 \\
\hline 3000.366 & 214.312 & 4612.796 & 157.336 & 346.440 & 46.896 \\
\hline 2980.068 & 130.441 & 4583.348 & 108.782 & 360.615 & 29.887 \\
\hline 4131.621 & 366.457 & 4825.806 & 83.871 & 518.098 & 74.497 \\
\hline 5250.599 & 179.918 & 4955.142 & 60.502 & 883.739 & 144.238 \\
\hline 6164.124 & 271.107 & 4987.733 & 71.423 & \#VALUE! & \#VALUE! \\
\hline 5498.965 & 139.992 & 4972.394 & 65.979 & 1293.145 & 187.114 \\
\hline 5217.785 & 301.677 & 4955.142 & 84.703 & 791.461 & 178.586 \\
\hline 5414.262 & 184.069 & 4979.233 & 83.685 & 619.729 & 214.629 \\
\hline 5857.055 & 532.460 & 4970.679 & 72.039 & 184.230 & 349.723 \\
\hline 5552.176 & 282.314 & 4970.679 & 66.036 & 1377.853 & 193.198 \\
\hline 5965.305 & 218.448 & 4989.427 & 65.416 & -84.562 & 347.716 \\
\hline 5384.886 & 296.169 & 4977.527 & 179.478 & 591.252 & 398.830 \\
\hline 5581.731 & 252.671 & 4979.233 & 77.707 & 1435.972 & 231.504 \\
\hline 4961.753 & 369.773 & 4942.932 & 115.660 & 737.620 & 229.594 \\
\hline 4398.688 & 363.141 & 4900.201 & 230.086 & 381.037 & 194.833 \\
\hline 5420.622 & 219.755 & 4982.640 & 77.574 & 508.575 & 208.014 \\
\hline 7034.125 & 572.946 & 4970.679 & 108.058 & 544.492 & 1382.989 \\
\hline 4406.663 & 180.458 & 4916.385 & 123.372 & 318.017 & 112.720 \\
\hline 6209.973 & 219.822 & 4996.180 & 82.973 & -741.349 & 576.880 \\
\hline 4892.793 & 254.999 & 4965.520 & 162.508 & 233.166 & 239.043 \\
\hline 5508.402 & 877.445 & 4921.737 & 92.283 & 3077.205 & 701.221 \\
\hline 4640.888 & 169.907 & 4935.904 & 103.849 & 338.205 & 124.154 \\
\hline 5891.373 & 464.169 & 4972.394 & 89.971 & 154.930 & 398.089 \\
\hline 5199.760 & 306.052 & 4949.923 & 97.057 & 949.728 & 202.766 \\
\hline 6371.287 & 1009.789 & 4972.394 & 203.934 & \#VALUE! & \#VALUE! \\
\hline 6751.266 & 446.582 & 4958.610 & 72.477 & \#VALUE! & \#VALUE! \\
\hline 5979.467 & 215.925 & 4972.394 & 101.967 & \#VALUE! & \#VALUE! \\
\hline 3473.625 & 246.440 & 4767.170 & 106.232 & 318.086 & 43.464 \\
\hline 5333.069 & 1010.476 & 4970.679 & 150.081 & 573.257 & 325.480 \\
\hline 3595.430 & 343.444 & 4757.143 & 146.784 & 382.988 & 66.532 \\
\hline 3748.161 & 258.824 & 4804.602 & 123.864 & 388.497 & 67.636 \\
\hline 5053.291 & 947.492 & 4951.665 & 90.912 & 472.564 & 169.855 \\
\hline 5208.812 & 434.068 & 4941.178 & 176.688 & 1043.501 & 316.779 \\
\hline 3737.982 & 202.836 & 4747.041 & 221.260 & 482.341 & 102.594 \\
\hline 5184.491 & 1106.446 & 4911.012 & 117.518 & 1826.333 & 456.493 \\
\hline 3970.169 & 503.375 & 4844.795 & 274.837 & 360.070 & 156.127 \\
\hline 4098.095 & 353.792 & 4802.658 & 254.489 & 568.703 & 164.696 \\
\hline 4229.186 & 380.226 & 4863.519 & 221.645 & 436.065 & 160.713 \\
\hline 4910.679 & 510.711 & 4942.932 & 146.096 & 484.710 & 216.178 \\
\hline 1791.084 & 992.747 & 4878.312 & 169.735 & 860.835 & 257.278 \\
\hline
\end{tabular}




\begin{tabular}{|c|c|c|c|c|c|}
\hline 4608.855 & 228.950 & 4907.418 & 136.317 & 520.849 & 151.930 \\
\hline 6359.748 & 801.037 & 4970.679 & 96.052 & 2370.845 & 715.484 \\
\hline 1662.236 & 357.341 & 3715.676 & 506.683 & 322.173 & 39.880 \\
\hline 3874.206 & 392.656 & 4850.440 & 293.193 & 314.020 & 153.177 \\
\hline 3693.543 & 329.424 & 4812.351 & 246.787 & 329.655 & 111.643 \\
\hline 4140.262 & 642.455 & 4869.086 & 189.459 & 374.217 & 137.519 \\
\hline 4502.596 & 389.180 & 4891.124 & 181.153 & 500.483 & 172.963 \\
\hline 4696.119 & 650.947 & 4930.609 & 251.124 & 389.706 & 283.413 \\
\hline 3880.894 & 243.098 & 4787.001 & 203.842 & 473.245 & 108.082 \\
\hline 5124.931 & 281.772 & 4912.805 & 234.826 & 1340.170 & 395.381 \\
\hline 5178.318 & 317.688 & 4892.944 & 218.435 & 1802.651 & 356.221 \\
\hline 6513.781 & 1494.966 & 4941.178 & 201.059 & 1074.127 & 1455.540 \\
\hline 3354.031 & 499.264 & 4641.615 & 289.219 & 458.049 & 103.077 \\
\hline 5725.090 & 1001.891 & 4935.904 & 195.481 & 1243.817 & 629.212 \\
\hline 5781.319 & 683.602 & 5007.915 & 176.750 & -78.036 & 636.027 \\
\hline 5214.803 & 833.134 & 4994.495 & 237.268 & -79.174 & 460.571 \\
\hline 4751.332 & 262.726 & 4951.665 & 133.337 & 312.718 & 182.080 \\
\hline 6752.581 & 717.243 & 4989.427 & 202.194 & -6296.547 & 6039.846 \\
\hline 3156.888 & 221.277 & 4710.033 & 273.045 & 306.919 & 75.734 \\
\hline 6088.696 & 607.351 & 4986.038 & 274.024 & \#VALUE! & \#VALUE! \\
\hline 4556.544 & 368.048 & 4896.577 & 105.906 & 519.000 & 127.968 \\
\hline 4844.072 & 703.840 & 4880.150 & 169.581 & 1332.373 & 338.274 \\
\hline 6885.371 & 1173.643 & 4960.341 & 126.724 & \#VALUE! & \#VALUE! \\
\hline 5196.724 & 469.584 & 4956.877 & 199.484 & 618.875 & 349.911 \\
\hline 2689.757 & 173.995 & 4498.277 & 128.307 & 328.440 & 27.526 \\
\hline 4581.365 & 498.306 & 4861.659 & 228.187 & 729.881 & 228.332 \\
\hline 4356.460 & 847.089 & 4880.150 & 213.546 & 547.493 & 220.887 \\
\hline 4503.800 & 534.624 & 4932.376 & 201.946 & 305.360 & 206.632 \\
\hline 1152.077 & 103.742 & 3086.418 & 275.808 & 315.161 & 14.895 \\
\hline 2548.205 & 338.257 & 4362.409 & 361.526 & 354.526 & 60.321 \\
\hline 5419.646 & 314.182 & 4994.495 & 166.088 & 83.010 & 421.849 \\
\hline 4706.025 & 645.925 & 4918.171 & 277.340 & 529.096 & 325.320 \\
\hline 4869.564 & 811.594 & 4930.609 & 165.374 & 926.873 & 559.754 \\
\hline 5151.975 & 285.500 & 4903.814 & 186.221 & 1478.449 & 294.783 \\
\hline 5255.775 & 806.284 & 4918.171 & 117.099 & 1350.722 & 266.628 \\
\hline 4936.144 & 350.382 & 4916.385 & 148.047 & 868.544 & 218.268 \\
\hline 5389.925 & 1072.622 & 4968.962 & 294.412 & 683.405 & 600.820 \\
\hline 5939.305 & 652.294 & 4930.609 & 226.624 & \#NUM! & \#VALUE! \\
\hline 5238.417 & 545.038 & 4923.516 & 116.788 & 1852.082 & 301.933 \\
\hline 4546.212 & 522.553 & 4876.472 & 81.799 & 618.905 & 107.063 \\
\hline 5548.312 & 176.998 & 4923.516 & 67.614 & 0.000 & 240.115 \\
\hline 6249.539 & 345.719 & 4956.877 & 60.450 & 0.000 & 455.124 \\
\hline 5852.285 & 167.735 & 4946.432 & 78.997 & \#VALUE! & \#VALUE! \\
\hline 6336.270 & 631.152 & 4986.038 & 101.270 & -157.902 & 678.122 \\
\hline 6562.451 & 358.884 & 4970.679 & 66.036 & \#NUM! & \#VALUE! \\
\hline 6684.682 & 305.533 & 4964.486 & 49.380 & 0.000 & 693.909 \\
\hline 4985.236 & 200.002 & 4919.955 & 104.680 & 888.694 & 164.368 \\
\hline 5640.740 & 168.609 & 4915.133 & 51.849 & 1521.573 & 193.744 \\
\hline 6031.282 & 141.669 & 4939.422 & 73.177 & 0.000 & 380.204 \\
\hline
\end{tabular}




\begin{tabular}{|c|c|c|c|c|c|}
\hline 5728.697 & 326.189 & 4930.609 & 73.500 & 1387.576 & 272.470 \\
\hline 6942.512 & 633.849 & 4946.257 & 54.695 & \#VALUE! & \#VALUE! \\
\hline 5389.925 & 294.971 & 4905.617 & 80.623 & 2922.426 & 278.614 \\
\hline 6028.874 & 574.178 & 4948.178 & 78.928 & \#VALUE! & \#VALUE! \\
\hline 6691.680 & 358.976 & 4944.683 & 55.346 & 0.000 & 666.599 \\
\hline 6546.485 & 1143.037 & 4962.070 & 78.380 & \#VALUE! & \#VALUE! \\
\hline 5703.174 & 333.032 & 4944.683 & 72.984 & 442.544 & 278.819 \\
\hline 5661.724 & 279.857 & 4940.652 & 53.630 & \#VALUE! & \#VALUE! \\
\hline 6351.983 & 415.322 & 4989.427 & 95.150 & -1227.326 & 813.690 \\
\hline 5788.134 & 213.656 & 4921.737 & 67.674 & 1747.613 & 270.125 \\
\hline 7169.088 & 289.473 & 4944.858 & 53.517 & \#VALUE! & \#VALUE! \\
\hline 6121.012 & 428.767 & 4975.818 & 59.877 & 374.538 & 360.980 \\
\hline 5499.417 & 245.510 & 4912.805 & 67.976 & 0.000 & 276.127 \\
\hline 5657.871 & 302.329 & 4930.609 & 79.625 & 578.401 & 282.946 \\
\hline 5654.003 & 433.257 & 4932.729 & 55.678 & 544.860 & 222.998 \\
\hline 5735.873 & 628.311 & 4935.904 & 61.088 & 1207.253 & 259.149 \\
\hline 4967.103 & 270.523 & 4889.301 & 150.055 & 1159.129 & 211.552 \\
\hline 6727.311 & 232.284 & 4969.133 & 33.043 & 0.000 & 581.774 \\
\hline 7174.304 & 613.188 & 4965.520 & 72.226 & 0.000 & 1299.774 \\
\hline 6088.696 & 531.432 & 4970.679 & 78.042 & 582.401 & 407.668 \\
\hline 4227.608 & 180.325 & 4846.679 & 140.483 & 502.842 & 105.131 \\
\hline 5764.080 & 277.310 & 4946.432 & 85.074 & 448.747 & 329.889 \\
\hline 5503.020 & 203.181 & 4925.292 & 67.554 & 0.000 & 238.677 \\
\hline 5866.530 & 273.286 & 4960.514 & 47.065 & \#VALUE! & \#VALUE! \\
\hline 6896.831 & 929.910 & 4982.640 & 65.640 & 845.538 & 822.089 \\
\hline 5811.631 & 285.818 & 4965.520 & 102.320 & 229.619 & 404.045 \\
\hline 5888.300 & 626.415 & 4953.404 & 72.666 & \#VALUE! & \#VALUE! \\
\hline 6512.118 & 769.906 & 4980.938 & 77.641 & 702.973 & 599.135 \\
\hline 5869.669 & 345.275 & 4944.683 & 72.984 & 1225.974 & 302.952 \\
\hline 5602.328 & 655.111 & 4918.171 & 129.426 & 666.328 & 394.236 \\
\hline 6154.701 & 661.487 & 4980.938 & 71.668 & 142.573 & 434.619 \\
\hline 6688.887 & 387.494 & 4949.923 & 103.123 & \#VALUE! & \#VALUE! \\
\hline 6443.252 & 713.400 & 4937.664 & 61.034 & 1003.917 & 478.158 \\
\hline 5942.227 & 787.730 & 4955.142 & 90.754 & 1035.941 & 405.027 \\
\hline 5598.242 & 430.634 & 4912.805 & 123.593 & \#VALUE! & \#VALUE! \\
\hline 6137.996 & 422.807 & 4962.760 & 59.669 & 2273.580 & 392.191 \\
\hline 5757.101 & 458.178 & 4934.141 & 67.256 & 1355.796 & 277.558 \\
\hline 5043.440 & 321.846 & 4896.577 & 62.297 & 1322.297 & 134.454 \\
\hline 4987.480 & 162.675 & 4896.577 & 62.297 & 1161.279 & 111.680 \\
\hline 5646.222 & 348.801 & 4960.341 & 72.414 & 3299.381 & 520.199 \\
\hline 4576.897 & 397.991 & 4894.762 & 74.824 & 586.516 & 102.741 \\
\hline 5888.300 & 304.259 & 4968.962 & 84.118 & 197.817 & 369.502 \\
\hline 4969.388 & 120.015 & 4919.955 & 80.049 & 846.700 & 132.374 \\
\hline 4454.500 & 162.696 & 4872.784 & 81.949 & 579.496 & 84.224 \\
\hline 5428.395 & 223.583 & 4930.609 & 67.375 & 2405.458 & 195.936 \\
\hline 5897.489 & 408.561 & 4941.529 & 37.159 & 1397.566 & 232.257 \\
\hline 5053.291 & 491.292 & 4911.012 & 111.333 & 1130.417 & 204.871 \\
\hline 4662.725 & 181.355 & 4902.008 & 86.981 & 608.115 & 109.602 \\
\hline 4763.515 & 291.104 & 4905.617 & 142.641 & 644.651 & 172.839 \\
\hline 1715.836 & 192.296 & 4912.805 & 86.515 & 568.263 & 113.533 \\
\hline
\end{tabular}




\begin{tabular}{|c|c|c|c|c|c|}
\hline 5503.020 & 137.086 & 4908.857 & 59.441 & 697.601 & 197.661 \\
\hline 2728.504 & 161.438 & 4507.998 & 180.320 & 343.417 & 34.886 \\
\hline 3663.717 & 122.464 & 4742.979 & 107.490 & 456.016 & 50.279 \\
\hline 4472.028 & 475.983 & 4872.784 & 94.556 & 559.815 & 105.564 \\
\hline 5349.940 & 188.300 & 4916.385 & 80.192 & 2274.218 & 208.190 \\
\hline 3913.686 & 626.867 & 4798.761 & 143.832 & 437.262 & 96.126 \\
\hline 5476.598 & 350.102 & 4958.610 & 138.914 & 1658.009 & 323.135 \\
\hline 6491.944 & 521.965 & 4937.664 & 67.138 & \#VALUE! & \#VALUE! \\
\hline 5693.901 & 132.124 & 4953.404 & 66.611 & 0.000 & 257.261 \\
\hline 5573.374 & 323.764 & 4930.609 & 85.750 & 0.000 & 351.792 \\
\hline 6020.804 & 208.721 & 4947.654 & 54.657 & 3106.476 & 495.533 \\
\hline 6140.399 & 232.811 & 4941.178 & 79.205 & \#VALUE! & \#VALUE! \\
\hline 7199.990 & 779.999 & 4955.142 & 127.055 & \#VALUE! & \#VALUE! \\
\hline 5739.442 & 282.930 & 4962.070 & 78.380 & 261.252 & 314.885 \\
\hline 5343.646 & 306.146 & 4923.516 & 116.788 & 2167.342 & 291.227 \\
\hline 6096.245 & 286.705 & 4902.008 & 68.342 & 1320.782 & 376.248 \\
\hline 5135.965 & 213.588 & 4927.067 & 92.037 & 1124.928 & 170.721 \\
\hline 6522.056 & 307.544 & 4968.962 & 78.109 & 0.000 & 657.380 \\
\hline 7319.491 & 759.058 & 4950.097 & 57.623 & \#VALUE! & \#VALUE! \\
\hline 6666.259 & 319.680 & 4942.932 & 97.398 & 0.000 & 871.509 \\
\hline 6209.973 & 206.083 & 4950.445 & 56.400 & \#NUM! & \#VALUE! \\
\hline 7114.480 & 399.907 & 4947.480 & 47.373 & 0.000 & 1046.334 \\
\hline 5364.476 & 208.010 & 4939.422 & 67.079 & 1702.488 & 170.393 \\
\hline 6137.996 & 233.273 & 4942.932 & 91.310 & 0.000 & 495.774 \\
\hline 4702.074 & 236.029 & 4883.818 & 112.848 & 747.834 & 135.259 \\
\hline 4859.444 & 274.060 & 4896.577 & 80.987 & 903.376 & 125.732 \\
\hline 5414.752 & 289.137 & 4930.609 & 61.250 & 2366.063 & 213.322 \\
\hline 5471.972 & 401.613 & 4946.432 & 85.074 & 2031.171 & 241.937 \\
\hline 4795.607 & 193.545 & 4916.385 & 67.855 & 631.295 & 103.269 \\
\hline 6464.406 & 478.433 & 4991.118 & 83.185 & \#VALUE! & \#VALUE! \\
\hline 5831.348 & 243.754 & 4972.394 & 77.975 & \#VALUE! & \#VALUE! \\
\hline 5511.083 & 153.221 & 4941.178 & 73.112 & 2805.842 & 237.815 \\
\hline 3250.775 & 113.094 & 4654.711 & 70.101 & 395.354 & 26.699 \\
\hline 4717.787 & 370.291 & 4896.577 & 62.297 & 693.415 & 100.448 \\
\hline 5178.318 & 381.226 & 4891.124 & 62.466 & 1918.645 & 183.106 \\
\hline 5377.789 & 211.212 & 4919.955 & 73.892 & 2457.337 & 201.044 \\
\hline 4988.973 & 258.496 & 4903.814 & 62.074 & 1076.689 & 118.529 \\
\hline 5346.274 & 166.637 & 4941.178 & 79.205 & 1575.284 & 176.862 \\
\hline 4035.192 & 108.224 & 4825.806 & 64.516 & 456.029 & 47.839 \\
\hline 6595.183 & 169.619 & 4982.640 & 77.574 & 382.865 & 701.468 \\
\hline 5292.948 & 147.832 & 4953.404 & 108.999 & 1019.942 & 222.521 \\
\hline 6637.246 & 288.995 & 4975.818 & 65.865 & 3177.747 & 1285.040 \\
\hline 5181.409 & 313.737 & 4903.814 & 62.074 & 1639.353 & 155.770 \\
\hline 5127.538 & 430.052 & 4918.171 & 117.099 & 1196.352 & 212.540 \\
\hline 5492.170 & 185.213 & 4941.178 & 146.225 & 2811.695 & 409.860 \\
\hline 5721.470 & 389.634 & 4948.178 & 72.857 & 408.994 & 283.488 \\
\hline 5259.782 & 270.877 & 4941.178 & 188.874 & 1060.752 & 333.538 \\
\hline 6369.373 & 626.101 & 4944.683 & 103.394 & \#NUM! & \#VALUE! \\
\hline 6075.987 & 230.151 & 4951.665 & 78.790 & 3303.166 & 944.639 \\
\hline 5171.485 & 188.461 & 4919.955 & 129.310 & 1336.083 & 223.248 \\
\hline
\end{tabular}




$\begin{array}{lrrrrr}6063.118 & 217.094 & 4956.877 & 78.585 & 1149.739 & 383.777 \\ 5634.438 & 242.105 & 4956.877 & 84.630 & 3177.038 & 395.950 \\ 5837.836 & 242.466 & 4955.142 & 133.105 & 865.667 & 477.770 \\ 5344.172 & 233.686 & 4951.665 & 157.581 & 1309.415 & 321.703 \\ 4436.663 & 244.585 & 4894.762 & 112.237 & 439.738 & 106.820\end{array}$




\begin{tabular}{|c|c|c|c|c|c|c|}
\hline Sample & ${ }^{207} \mathrm{~Pb} /{ }^{235} \mathrm{U}$ & Error $2 \sigma$ & ${ }^{206} \mathrm{~Pb} /{ }^{238} \mathrm{U}$ & Error $2 \sigma$ & orrelation_6_38 & ${ }^{238} \mathrm{U} /{ }^{206} \mathrm{~Pb}$ \\
\hline BLR_1 & 2.263 & 0.056 & 0.178 & 0.003 & 0.351 & 5.618 \\
\hline BLR_2 & 2.130 & 0.055 & 0.176 & 0.003 & 0.319 & 5.688 \\
\hline BLR_3 & 2.108 & 0.056 & 0.178 & 0.003 & 0.156 & 5.624 \\
\hline BLR_4 & 2.091 & 0.077 & 0.176 & 0.003 & 0.366 & 5.698 \\
\hline BLR_5 & 2.242 & 0.059 & 0.178 & 0.004 & 0.559 & 5.609 \\
\hline BLR_6 & 2.124 & 0.061 & 0.175 & 0.003 & 0.516 & 5.727 \\
\hline BLR_7 & 2.210 & 0.058 & 0.178 & 0.003 & 0.199 & 5.615 \\
\hline BLR_8 & 2.181 & 0.067 & 0.182 & 0.003 & 0.298 & 5.501 \\
\hline BLR_9 & 2.209 & 0.045 & 0.178 & 0.002 & 0.245 & 5.624 \\
\hline BLR_10 & 2.223 & 0.050 & 0.179 & 0.002 & 0.218 & 5.583 \\
\hline BLR_11 & 2.062 & 0.051 & 0.174 & 0.003 & 0.128 & 5.741 \\
\hline BLR_12 & 2.158 & 0.057 & 0.178 & 0.003 & 0.182 & 5.618 \\
\hline BLR_13 & 2.256 & 0.061 & 0.182 & 0.003 & 0.362 & 5.488 \\
\hline BLR_14 & 2.217 & 0.064 & 0.176 & 0.003 & 0.422 & 5.672 \\
\hline BLR_15 & 2.103 & 0.058 & 0.172 & 0.002 & 0.418 & 5.804 \\
\hline BLR_16 & 2.182 & 0.063 & 0.175 & 0.003 & 0.344 & 5.718 \\
\hline BLR_17 & 2.228 & 0.053 & 0.176 & 0.003 & 0.115 & 5.688 \\
\hline BLR_18 & 2.290 & 0.077 & 0.181 & 0.002 & 0.203 & 5.522 \\
\hline BLR_19 & 2.165 & 0.088 & 0.180 & 0.003 & -0.008 & 5.546 \\
\hline BLR_20 & 2.203 & 0.057 & 0.183 & 0.003 & 0.344 & 5.476 \\
\hline T_MKED1_1 & 3.442 & 0.083 & 0.266 & 0.006 & 0.094 & 3.759 \\
\hline T_MKED1_2 & 3.520 & 0.110 & 0.264 & 0.005 & 0.641 & 3.788 \\
\hline T_MKED1_3 & 3.360 & 0.120 & 0.269 & 0.006 & 0.359 & 3.713 \\
\hline T_MKED1_4 & 3.520 & 0.120 & 0.273 & 0.005 & 0.572 & 3.660 \\
\hline T_MKED1_5 & 3.610 & 0.140 & 0.270 & 0.006 & 0.408 & 3.698 \\
\hline T_MKED1_6 & 3.500 & 0.120 & 0.266 & 0.007 & 0.662 & 3.759 \\
\hline T_MKED1_7 & 3.620 & 0.160 & 0.269 & 0.006 & 0.602 & 3.723 \\
\hline T_MKED1_8 & 3.499 & 0.093 & 0.272 & 0.005 & 0.253 & 3.671 \\
\hline T_MKED1_9 & 3.610 & 0.140 & 0.274 & 0.005 & 0.488 & 3.647 \\
\hline T_MKED1_10 & 3.569 & 0.097 & 0.273 & 0.006 & 0.561 & 3.667 \\
\hline T_MKED1_11 & 3.550 & 0.120 & 0.273 & 0.004 & 0.318 & 3.660 \\
\hline T_MKED1_12 & 3.596 & 0.093 & 0.273 & 0.004 & 0.089 & 3.668 \\
\hline T_MKED1_13 & 3.600 & 0.110 & 0.273 & 0.004 & 0.077 & 3.670 \\
\hline T_MKED1_14 & 3.550 & 0.110 & 0.270 & 0.006 & 0.343 & 3.705 \\
\hline T_MKED1_15 & 3.630 & 0.120 & 0.271 & 0.008 & 0.505 & 3.686 \\
\hline T_MKED1_16 & 3.480 & 0.140 & 0.272 & 0.005 & 0.311 & 3.676 \\
\hline T_MKED1_17 & 3.610 & 0.130 & 0.274 & 0.007 & 0.556 & 3.644 \\
\hline T_MKED1_18 & 3.514 & 0.084 & 0.270 & 0.004 & 0.277 & 3.702 \\
\hline T_MKED1_19 & 3.350 & 0.150 & 0.263 & 0.004 & 0.298 & 3.804 \\
\hline T_MKED1_20 & 3.470 & 0.130 & 0.266 & 0.005 & 0.476 & 3.766 \\
\hline ZA16_77_1 & 31.500 & 5.400 & 0.295 & 0.046 & 0.818 & 3.390 \\
\hline ZA16_77_2 & 16.000 & 2.600 & 0.181 & 0.018 & 0.752 & 5.525 \\
\hline ZA16_77_3 & 48.100 & 8.300 & 0.477 & 0.078 & 0.737 & 2.096 \\
\hline ZA16_77_4 & 105.000 & 18.000 & 0.880 & 0.150 & 0.527 & 1.136 \\
\hline ZA16_77_5 & 187.000 & 29.000 & 1.680 & 0.240 & 0.934 & 0.595 \\
\hline ZA16_77_6 & 164.000 & 45.000 & 1.480 & 0.430 & 0.893 & 0.676 \\
\hline ZA16_77_7 & 135.000 & 27.000 & 1.330 & 0.280 & 0.923 & 0.752 \\
\hline
\end{tabular}




\begin{tabular}{|c|c|c|c|c|c|c|}
\hline ZA16_77_8 & 87.000 & 18.000 & 0.675 & 0.095 & 0.818 & 1.481 \\
\hline ZA16_77_9 & 35.200 & 5.000 & 0.318 & 0.037 & 0.728 & 3.145 \\
\hline ZA16_77_10 & 30.300 & 3.100 & 0.316 & 0.036 & 0.799 & 3.165 \\
\hline ZA16_77_11 & 27.100 & 4.400 & 0.264 & 0.038 & 0.591 & 3.788 \\
\hline ZA16_77_12 & 26.300 & 2.400 & 0.289 & 0.027 & 0.572 & 3.460 \\
\hline ZA16_77_13 & 51.300 & 9.100 & 0.496 & 0.065 & 0.685 & 2.016 \\
\hline ZA16_77_14 & 32.000 & 4.000 & 0.326 & 0.034 & 0.845 & 3.067 \\
\hline ZA16_77_15 & 32.300 & 4.800 & 0.346 & 0.039 & 0.390 & 2.890 \\
\hline ZA16_77_16 & 21.300 & 3.700 & 0.210 & 0.032 & 0.832 & 4.762 \\
\hline ZA16_77_17 & 86.000 & 74.000 & 1.700 & 1.500 & 0.964 & 0.588 \\
\hline ZA16_77_18 & 11.800 & 1.500 & 0.156 & 0.017 & 0.834 & 6.410 \\
\hline ZA16_77_19 & 10.300 & 6.000 & 0.164 & 0.074 & 0.974 & 6.098 \\
\hline ZA16_77_20 & 21.500 & 2.200 & 0.238 & 0.019 & 0.765 & 4.202 \\
\hline ZA16_77_21 & 17.000 & 2.000 & 0.191 & 0.017 & 0.877 & 5.236 \\
\hline ZA16_77_22 & 14.500 & 2.000 & 0.195 & 0.016 & 0.459 & 5.128 \\
\hline ZA16_77_23 & 13.300 & 1.000 & 0.167 & 0.017 & 0.094 & 5.988 \\
\hline ZA16_77_24 & 43.000 & 13.000 & 0.460 & 0.130 & 0.937 & 2.174 \\
\hline ZA16_77_25 & 7.520 & 0.970 & 0.131 & 0.012 & 0.610 & 7.634 \\
\hline ZA16_77_26 & 6.680 & 0.860 & 0.101 & 0.009 & 0.693 & 9.891 \\
\hline ZA16_77_27 & 40.900 & 5.300 & 0.403 & 0.049 & 0.670 & 2.481 \\
\hline ZA16_77_28 & 15.000 & 2.000 & 0.171 & 0.015 & 0.293 & 5.848 \\
\hline ZA16_77_29 & 30.000 & 12.000 & 0.291 & 0.098 & 0.974 & 3.436 \\
\hline ZA16_77_30 & 15.100 & 1.600 & 0.191 & 0.018 & 0.435 & 5.236 \\
\hline ZA16_77_31 & 67.000 & 4.100 & 0.626 & 0.037 & 0.187 & 1.597 \\
\hline ZA16_77_32 & 86.000 & 15.000 & 0.870 & 0.130 & 0.768 & 1.149 \\
\hline ZA16_77_33 & 49.300 & 6.600 & 0.471 & 0.067 & 0.676 & 2.123 \\
\hline ZA16_77_34 & 1.750 & 0.750 & 0.065 & 0.011 & 0.987 & 15.385 \\
\hline ZA16_77_35 & 39.300 & 5.100 & 0.412 & 0.054 & 0.587 & 2.427 \\
\hline ZA16_77_36 & 116.000 & 31.000 & 1.220 & 0.330 & 0.860 & 0.820 \\
\hline ZA16_77_37 & 19.700 & 2.500 & 0.221 & 0.026 & 0.766 & 4.525 \\
\hline ZA16_77_38 & 83.000 & 25.000 & 0.690 & 0.200 & 0.802 & 1.449 \\
\hline ZA16_77_39 & 104.000 & 21.000 & 0.950 & 0.170 & 0.498 & 1.053 \\
\hline ZA16_77_40 & 80.000 & 12.000 & 0.770 & 0.120 & 0.733 & 1.299 \\
\hline ZA16_77_41 & 65.000 & 15.000 & 0.520 & 0.120 & 0.867 & 1.923 \\
\hline ZA16_77_42 & 61.000 & 14.000 & 0.580 & 0.140 & 0.797 & 1.724 \\
\hline ZA16_77_43 & 17.300 & 2.800 & 0.180 & 0.019 & 0.900 & 5.556 \\
\hline ZA16_77_44 & 30.700 & 9.600 & 0.287 & 0.068 & 0.953 & 3.484 \\
\hline ZA16_77_45 & 38.800 & 4.500 & 0.353 & 0.027 & 0.455 & 2.833 \\
\hline ZA16_77_46 & 55.200 & 4.000 & 0.491 & 0.043 & 0.482 & 2.037 \\
\hline ZA16_78_1 & 59.200 & 3.300 & 0.549 & 0.030 & 0.715 & 1.821 \\
\hline ZA16_78_2 & 249.000 & 42.000 & 2.230 & 0.370 & 0.965 & 0.448 \\
\hline ZA16_78_3 & 283.000 & 45.000 & 2.470 & 0.390 & 0.981 & 0.405 \\
\hline ZA16_78_4 & 106.000 & 26.000 & 0.900 & 0.220 & 0.987 & 1.111 \\
\hline ZA16_78_5 & 104.600 & 9.300 & 0.927 & 0.081 & 0.905 & 1.079 \\
\hline ZA16_78_6 & 48.600 & 9.700 & 0.445 & 0.075 & 0.935 & 2.247 \\
\hline ZA16_78_7 & 175.000 & 40.000 & 1.510 & 0.220 & 0.856 & 0.662 \\
\hline ZA16_78_8 & 3.750 & 0.420 & 0.037 & 0.004 & 0.012 & 27.322 \\
\hline ZA16_78_9 & 68.000 & 12.000 & 0.710 & 0.130 & 0.964 & 1.408 \\
\hline ZA16 $78 \quad 10$ & 25.100 & 2.700 & 0.256 & 0.024 & 0.737 & 3.906 \\
\hline
\end{tabular}




\begin{tabular}{|c|c|c|c|c|c|c|}
\hline ZA16_78_11 & 29.000 & 14.000 & 0.270 & 0.120 & 0.982 & 3.704 \\
\hline ZA16_78_12 & 22.300 & 3.400 & 0.212 & 0.018 & 0.890 & 4.717 \\
\hline ZA16_78_13 & 67.000 & 15.000 & 0.620 & 0.130 & 0.952 & 1.613 \\
\hline ZA16_78_14 & 45.600 & 3.800 & 0.434 & 0.036 & 0.531 & 2.304 \\
\hline ZA16_78_15 & 55.000 & 17.000 & 0.530 & 0.150 & 0.967 & 1.887 \\
\hline ZA16_78_16 & 153.000 & 28.000 & 1.300 & 0.170 & 0.780 & 0.769 \\
\hline ZA16_78_17 & 536.000 & 50.000 & 4.660 & 0.460 & 0.918 & 0.215 \\
\hline ZA16_78_18 & 14.730 & 0.960 & 0.180 & 0.011 & 0.819 & 5.556 \\
\hline ZA16_78_19 & 20.100 & 2.400 & 0.222 & 0.028 & 0.919 & 4.505 \\
\hline ZA16_78_20 & 33.400 & 1.700 & 0.343 & 0.018 & 0.814 & 2.915 \\
\hline ZA16_78_21 & 610.000 & 110.000 & 5.600 & 1.000 & 0.976 & 0.179 \\
\hline ZA16_78_22 & 4.900 & 1.200 & 0.086 & 0.012 & 0.966 & 11.628 \\
\hline ZA16_78_23 & 18.700 & 1.700 & 0.201 & 0.015 & 0.932 & 4.975 \\
\hline ZA16_78_24 & 349.000 & 53.000 & 2.980 & 0.440 & 0.984 & 0.336 \\
\hline ZA16_78_25 & 42.000 & 15.000 & 0.400 & 0.110 & 0.898 & 2.500 \\
\hline ZA16_78_26 & 21.100 & 3.100 & 0.229 & 0.030 & 0.843 & 4.367 \\
\hline ZA16_78_27 & 81.000 & 8.600 & 0.718 & 0.069 & 0.936 & 1.393 \\
\hline ZA16_78_28 & 24.000 & 4.300 & 0.248 & 0.035 & 0.779 & 4.032 \\
\hline ZA16_78_29 & 28.900 & 1.800 & 0.285 & 0.014 & 0.607 & 3.509 \\
\hline ZA16_78_30 & 92.000 & 10.000 & 0.794 & 0.097 & 0.821 & 1.259 \\
\hline ZA16_78_31 & 14.000 & 1.400 & 0.179 & 0.016 & 0.472 & 5.587 \\
\hline ZA16_78_32 & 44.500 & 8.300 & 0.415 & 0.075 & 0.825 & 2.410 \\
\hline ZA16_78_33 & 23.800 & 2.200 & 0.254 & 0.018 & 0.625 & 3.937 \\
\hline ZA16_78_34 & 43.700 & 6.300 & 0.410 & 0.058 & 0.681 & 2.439 \\
\hline ZA16_78_35 & 66.000 & 7.900 & 0.655 & 0.089 & 0.971 & 1.527 \\
\hline ZA16_117_1 & 101.000 & 18.000 & 0.920 & 0.150 & 0.991 & 1.087 \\
\hline ZA16_117_2 & 142.000 & 21.000 & 1.280 & 0.190 & 0.985 & 0.781 \\
\hline ZA16_117_3 & 135.000 & 37.000 & 1.170 & 0.320 & 0.993 & 0.855 \\
\hline ZA16_117_4 & 51.900 & 2.500 & 0.502 & 0.028 & 0.837 & 1.992 \\
\hline ZA16_117_5 & 162.000 & 57.000 & 1.470 & 0.510 & 0.996 & 0.680 \\
\hline ZA16_117_6 & 191.200 & 9.000 & 1.750 & 0.084 & 0.914 & 0.571 \\
\hline ZA16_117_7 & 182.000 & 33.000 & 1.630 & 0.260 & 0.982 & 0.613 \\
\hline ZA16_117_8 & 880.000 & 240.000 & 8.500 & 2.400 & 0.995 & 0.118 \\
\hline ZA16_117_9 & 38.200 & 3.900 & 0.373 & 0.034 & 0.928 & 2.681 \\
\hline ZA16_117_10 & 33.600 & 5.200 & 0.328 & 0.038 & 0.933 & 3.049 \\
\hline ZA16_117_11 & 275.000 & 27.000 & 2.410 & 0.220 & 0.979 & 0.415 \\
\hline ZA16_117_12 & 100.000 & 14.000 & 0.890 & 0.110 & 0.985 & 1.124 \\
\hline ZA16_117_13 & 156.000 & 87.000 & 1.390 & 0.740 & 0.998 & 0.719 \\
\hline ZA16_117_14 & 205.000 & 11.000 & 1.820 & 0.100 & 0.941 & 0.549 \\
\hline ZA16_117_15 & 37.000 & 8.900 & 0.386 & 0.087 & 0.976 & 2.591 \\
\hline ZA16_117_16 & 56.400 & 6.400 & 0.544 & 0.062 & 0.896 & 1.838 \\
\hline ZA16_117_17 & 170.000 & 46.000 & 1.570 & 0.410 & 0.995 & 0.637 \\
\hline ZA16_117_18 & 24.600 & 2.400 & 0.259 & 0.022 & 0.897 & 3.861 \\
\hline ZA16_117_19 & 57.300 & 7.700 & 0.562 & 0.074 & 0.953 & 1.779 \\
\hline ZA16_117_20 & 39.800 & 3.900 & 0.372 & 0.029 & 0.934 & 2.688 \\
\hline ZA16_117_21 & 57.800 & 9.200 & 0.547 & 0.072 & 0.982 & 1.828 \\
\hline ZA16_117_22 & 104.000 & 11.000 & 0.960 & 0.089 & 0.985 & 1.042 \\
\hline ZA16_117_23 & 88.000 & 9.800 & 0.778 & 0.080 & 0.960 & 1.285 \\
\hline ZA16_117_24 & 218.000 & 26.000 & 1.950 & 0.240 & 0.985 & 0.513 \\
\hline
\end{tabular}




\begin{tabular}{|c|c|c|c|c|c|c|}
\hline ZA16_117_25 & 59.800 & 4.600 & 0.560 & 0.041 & 0.895 & 1.786 \\
\hline ZA16_117_26 & 151.000 & 15.000 & 1.350 & 0.130 & 0.974 & 0.741 \\
\hline ZA16_117_27 & 227.000 & 20.000 & 2.040 & 0.170 & 0.967 & 0.490 \\
\hline ZA16_117_28 & 156.700 & 8.000 & 1.400 & 0.078 & 0.858 & 0.714 \\
\hline ZA16_117_29 & 93.300 & 5.000 & 0.856 & 0.042 & 0.826 & 1.168 \\
\hline ZA184_1 & 157.000 & 16.000 & 1.440 & 0.130 & 0.760 & 0.694 \\
\hline ZA184_2 & 124.000 & 11.000 & 1.052 & 0.091 & 0.822 & 0.951 \\
\hline ZA184_3 & 494.000 & 73.000 & 4.390 & 0.640 & 0.939 & 0.228 \\
\hline ZA184_4 & 105.000 & 31.000 & 0.860 & 0.250 & 0.895 & 1.163 \\
\hline ZA184_5 & 255.000 & 27.000 & 1.960 & 0.240 & 0.588 & 0.510 \\
\hline ZA184_6 & 27.100 & 6.700 & 0.279 & 0.056 & 0.930 & 3.584 \\
\hline ZA184_7 & 161.000 & 14.000 & 1.390 & 0.120 & 0.814 & 0.719 \\
\hline ZA184_8 & 27.900 & 2.600 & 0.288 & 0.030 & 0.681 & 3.472 \\
\hline ZA184_9 & 29.400 & 2.700 & 0.311 & 0.030 & 0.746 & 3.215 \\
\hline ZA184_10 & 99.000 & 21.000 & 0.890 & 0.200 & 0.901 & 1.124 \\
\hline ZA184_11 & 173.000 & 35.000 & 1.460 & 0.280 & 0.937 & 0.685 \\
\hline ZA184_12 & 119.000 & 10.000 & 1.095 & 0.066 & 0.485 & 0.913 \\
\hline ZA184_13 & 505.000 & 29.000 & 4.400 & 0.270 & 0.862 & 0.227 \\
\hline ZA184_14 & 51.800 & 5.200 & 0.434 & 0.038 & 0.687 & 2.304 \\
\hline ZA184_15 & 82.000 & 14.000 & 0.730 & 0.120 & 0.855 & 1.370 \\
\hline ZA184_16 & 65.200 & 6.800 & 0.669 & 0.078 & 0.567 & 1.495 \\
\hline ZA184_17 & 41.900 & 4.900 & 0.397 & 0.035 & 0.540 & 2.519 \\
\hline ZA184_18 & 91.600 & 9.800 & 0.815 & 0.074 & 0.593 & 1.227 \\
\hline ZA184_19 & 55.900 & 4.200 & 0.498 & 0.032 & 0.610 & 2.008 \\
\hline ZA184_20 & 7.800 & 1.600 & 0.112 & 0.013 & 0.746 & 8.929 \\
\hline ZA184_21 & 13.100 & 1.500 & 0.162 & 0.015 & 0.950 & 6.173 \\
\hline ZA184_22 & 48.600 & 7.100 & 0.451 & 0.056 & 0.768 & 2.217 \\
\hline ZA184_23 & 14.700 & 2.300 & 0.176 & 0.021 & 0.825 & 5.682 \\
\hline ZA184_24 & 153.000 & 16.000 & 1.360 & 0.200 & 0.860 & 0.735 \\
\hline ZA184_25 & 87.000 & 13.000 & 0.820 & 0.120 & 0.942 & 1.220 \\
\hline
\end{tabular}




\begin{tabular}{|c|c|c|c|c|c|c|}
\hline Error $2 \sigma$ & ${ }^{207} \mathrm{~Pb} /{ }^{206} \mathrm{~Pb}$ & Error $2 \sigma$ & Jrrelation_38 & |Age ${ }^{206} \mathrm{~Pb} /{ }^{23}$ & Error $2 \sigma$ & FinalAge ${ }^{207} \mathrm{~Pb}$ \\
\hline 0.092 & 0.092 & 0.003 & 0.240 & 1056.039 & 17.205 & 1200.840 \\
\hline 0.097 & 0.089 & 0.002 & 0.407 & 1043.989 & 17.816 & 1158.586 \\
\hline 0.095 & 0.086 & 0.002 & 0.409 & 1054.945 & 17.800 & 1151.423 \\
\hline 0.097 & 0.086 & 0.003 & 0.110 & 1042.344 & 17.818 & 1145.854 \\
\hline 0.120 & 0.090 & 0.002 & 0.243 & 1057.681 & 22.542 & 1194.284 \\
\hline 0.085 & 0.088 & 0.002 & 0.039 & 1037.406 & 15.448 & 1156.637 \\
\hline 0.079 & 0.089 & 0.002 & 0.339 & 1056.586 & 14.831 & 1184.212 \\
\hline 0.088 & 0.087 & 0.003 & 0.215 & 1076.801 & 17.177 & 1174.997 \\
\hline 0.076 & 0.091 & 0.002 & 0.359 & 1054.945 & 14.240 & 1183.895 \\
\hline 0.065 & 0.090 & 0.002 & 0.372 & 1062.056 & 12.453 & 1188.316 \\
\hline 0.092 & 0.086 & 0.002 & 0.401 & 1035.211 & 16.639 & 1136.283 \\
\hline 0.088 & 0.088 & 0.002 & 0.376 & 1056.039 & 16.612 & 1167.628 \\
\hline 0.090 & 0.090 & 0.003 & 0.114 & 1078.982 & 17.766 & 1198.659 \\
\hline 0.084 & 0.091 & 0.002 & 0.314 & 1046.730 & 15.437 & 1186.424 \\
\hline 0.081 & 0.088 & 0.002 & 0.150 & 1024.771 & 14.274 & 1149.789 \\
\hline 0.101 & 0.089 & 0.003 & 0.221 & 1039.053 & 18.417 & 1175.316 \\
\hline 0.084 & 0.092 & 0.003 & 0.435 & 1043.989 & 15.440 & 1189.890 \\
\hline 0.073 & 0.092 & 0.003 & 0.198 & 1072.981 & 14.220 & 1209.207 \\
\hline 0.086 & 0.088 & 0.004 & 0.352 & 1068.613 & 16.595 & 1169.877 \\
\hline 0.075 & 0.088 & 0.002 & 0.129 & 1081.163 & 14.802 & 1181.995 \\
\hline 0.078 & 0.093 & 0.003 & 0.554 & 1520.466 & 31.438 & 1514.042 \\
\hline 0.070 & 0.097 & 0.003 & -0.266 & 1510.274 & 28.032 & 1531.718 \\
\hline 0.076 & 0.092 & 0.003 & 0.217 & 1537.248 & 31.396 & 1495.123 \\
\hline 0.064 & 0.094 & 0.003 & -0.005 & 1557.024 & 27.356 & 1531.718 \\
\hline 0.078 & 0.096 & 0.003 & 0.283 & 1542.832 & 32.523 & 1551.737 \\
\hline 0.095 & 0.096 & 0.002 & 0.037 & 1520.466 & 38.297 & 1527.215 \\
\hline 0.078 & 0.097 & 0.004 & 0.052 & 1533.692 & 31.976 & 1553.937 \\
\hline 0.067 & 0.093 & 0.002 & 0.498 & 1552.973 & 28.505 & 1526.989 \\
\hline 0.068 & 0.095 & 0.003 & 0.115 & 1562.086 & 29.054 & 1551.737 \\
\hline 0.074 & 0.094 & 0.002 & 0.219 & 1554.492 & 31.352 & 1542.666 \\
\hline 0.054 & 0.094 & 0.003 & 0.151 & 1557.024 & 22.797 & 1538.435 \\
\hline 0.052 & 0.096 & 0.003 & 0.345 & 1553.986 & 22.232 & 1548.648 \\
\hline 0.057 & 0.097 & 0.003 & 0.353 & 1553.479 & 23.944 & 1549.532 \\
\hline 0.085 & 0.096 & 0.003 & 0.344 & 1540.294 & 35.383 & 1538.435 \\
\hline 0.109 & 0.098 & 0.003 & 0.322 & 1547.397 & 45.629 & 1556.132 \\
\hline 0.065 & 0.094 & 0.003 & 0.100 & 1550.946 & 27.370 & 1522.692 \\
\hline 0.086 & 0.097 & 0.003 & 0.089 & 1563.097 & 37.027 & 1551.737 \\
\hline 0.055 & 0.093 & 0.002 & 0.330 & 1541.310 & 22.826 & 1530.369 \\
\hline 0.064 & 0.095 & 0.003 & 0.202 & 1504.662 & 25.183 & 1492.792 \\
\hline 0.077 & 0.095 & 0.003 & 0.064 & 1517.920 & 30.873 & 1520.423 \\
\hline 0.529 & 0.690 & 0.085 & 0.398 & 1666.467 & 259.856 & 3534.792 \\
\hline 0.549 & 0.680 & 0.110 & 0.193 & 1072.435 & 106.651 & 2876.797 \\
\hline 0.343 & 0.740 & 0.120 & 0.521 & 2514.185 & 411.125 & 3953.758 \\
\hline 0.194 & 0.810 & 0.160 & 0.593 & 4069.439 & 693.654 & 4735.177 \\
\hline 0.085 & 0.825 & 0.043 & -0.039 & 6354.983 & 907.855 & 5316.994 \\
\hline 0.196 & 0.816 & 0.093 & 0.321 & 5855.011 & 1701.118 & 5184.491 \\
\hline 0.158 & 0.776 & 0.054 & -0.113 & 5452.817 & 1147.962 & 4988.227 \\
\hline
\end{tabular}




\begin{tabular}{|c|c|c|c|c|c|c|}
\hline 0.209 & 0.828 & 0.087 & -0.272 & 3325.145 & 467.983 & 4546.212 \\
\hline 0.366 & 0.783 & 0.079 & 0.289 & 1779.954 & 207.102 & 3644.270 \\
\hline 0.361 & 0.712 & 0.051 & 0.370 & 1770.165 & 201.664 & 3496.591 \\
\hline 0.545 & 0.750 & 0.084 & 0.162 & 1510.274 & 217.388 & 3387.084 \\
\hline 0.323 & 0.668 & 0.062 & 0.356 & 1636.530 & 152.894 & 3357.757 \\
\hline 0.264 & 0.750 & 0.100 & 0.070 & 2596.583 & 340.278 & 4017.867 \\
\hline 0.320 & 0.708 & 0.049 & 0.179 & 1818.965 & 189.708 & 3550.295 \\
\hline 0.326 & 0.690 & 0.110 & 0.332 & 1915.470 & 215.906 & 3559.484 \\
\hline 0.726 & 0.699 & 0.073 & 0.185 & 1228.818 & 187.248 & 3152.345 \\
\hline 0.519 & 0.715 & 0.064 & 0.199 & 6402.912 & 5649.629 & 4534.607 \\
\hline 0.699 & 0.545 & 0.049 & 0.252 & 934.509 & 101.838 & 2588.663 \\
\hline 2.751 & 0.444 & 0.080 & -0.805 & 978.968 & 441.729 & 2462.104 \\
\hline 0.335 & 0.657 & 0.038 & 0.208 & 1376.291 & 109.872 & 3161.411 \\
\hline 0.466 & 0.619 & 0.033 & 0.104 & 1126.790 & 100.290 & 2934.835 \\
\hline 0.421 & 0.518 & 0.064 & 0.234 & 1148.404 & 94.228 & 2783.003 \\
\hline 0.610 & 0.571 & 0.063 & 0.726 & 995.561 & 101.345 & 2701.182 \\
\hline 0.614 & 0.722 & 0.069 & -0.370 & 2439.558 & 689.440 & 3842.402 \\
\hline 0.699 & 0.465 & 0.060 & -0.074 & 793.568 & 72.693 & 2175.373 \\
\hline 0.910 & 0.478 & 0.058 & 0.348 & 620.852 & 57.111 & 2069.980 \\
\hline 0.302 & 0.763 & 0.074 & 0.136 & 2182.838 & 265.407 & 3792.746 \\
\hline 0.513 & 0.643 & 0.082 & 0.365 & 1017.619 & 89.265 & 2815.240 \\
\hline 1.157 & 0.625 & 0.049 & -0.579 & 1646.524 & 554.500 & 3486.812 \\
\hline 0.493 & 0.612 & 0.071 & 0.355 & 1126.790 & 106.190 & 2821.566 \\
\hline 0.094 & 0.767 & 0.052 & 0.651 & 3133.750 & 185.222 & 4284.417 \\
\hline 0.172 & 0.760 & 0.100 & -0.023 & 4035.058 & 602.940 & 4534.607 \\
\hline 0.302 & 0.781 & 0.092 & 0.661 & 2487.945 & 353.911 & 3978.276 \\
\hline 2.604 & 0.165 & 0.031 & -0.891 & 405.962 & 68.701 & 1027.162 \\
\hline 0.318 & 0.722 & 0.083 & 0.336 & 2224.059 & 291.503 & 3753.213 \\
\hline 0.222 & 0.737 & 0.086 & 0.137 & 5141.062 & 1390.615 & 4835.431 \\
\hline 0.532 & 0.689 & 0.057 & 0.184 & 1287.157 & 151.430 & 3076.746 \\
\hline 0.420 & 0.789 & 0.097 & -0.099 & 3382.617 & 980.469 & 4498.976 \\
\hline 0.188 & 0.800 & 0.120 & 0.046 & 4305.105 & 770.387 & 4725.552 \\
\hline 0.202 & 0.758 & 0.086 & 0.558 & 3680.771 & 573.627 & 4462.049 \\
\hline 0.444 & 0.801 & 0.094 & -0.017 & 2699.180 & 622.888 & 4254.104 \\
\hline 0.416 & 0.770 & 0.100 & 0.410 & 2948.750 & 711.767 & 4190.622 \\
\hline 0.586 & 0.663 & 0.044 & -0.355 & 1066.975 & 112.625 & 2951.618 \\
\hline 0.826 & 0.614 & 0.084 & -0.446 & 1626.520 & 385.378 & 3509.485 \\
\hline 0.217 & 0.798 & 0.082 & 0.340 & 1948.908 & 149.067 & 3740.536 \\
\hline 0.178 & 0.818 & 0.061 & 0.575 & 2575.001 & 225.509 & 4090.894 \\
\hline 0.100 & 0.759 & 0.035 & 0.304 & 2821.012 & 154.154 & 4160.707 \\
\hline 0.074 & 0.828 & 0.032 & -0.140 & 7558.305 & 1254.069 & 5606.398 \\
\hline 0.064 & 0.874 & 0.025 & -0.301 & 8020.336 & 1266.369 & 5735.873 \\
\hline 0.272 & 0.800 & 0.027 & -0.372 & 4137.656 & 1011.427 & 4744.711 \\
\hline 0.094 & 0.819 & 0.025 & 0.051 & 4228.618 & 369.491 & 4731.338 \\
\hline 0.379 & 0.729 & 0.051 & 0.003 & 2372.985 & 399.941 & 3964.046 \\
\hline 0.096 & 0.816 & 0.071 & -0.122 & 5932.524 & 864.341 & 5250.022 \\
\hline 3.210 & 0.750 & 0.120 & 0.670 & 231.724 & 27.224 & 1582.114 \\
\hline 0.258 & 0.713 & 0.058 & 0.343 & 3458.458 & 633.239 & 4299.240 \\
\hline 0.366 & 0.703 & 0.055 & 0.005 & 1469.345 & 137.751 & 3312.114 \\
\hline
\end{tabular}




\begin{tabular}{|c|c|c|c|c|c|c|}
\hline 1.646 & 0.704 & 0.083 & -0.493 & 1540.802 & 684.801 & 3453.518 \\
\hline 0.400 & 0.718 & 0.054 & -0.112 & 1239.464 & 105.238 & 3196.886 \\
\hline 0.338 & 0.732 & 0.042 & -0.270 & 3109.919 & 652.080 & 4284.417 \\
\hline 0.191 & 0.771 & 0.063 & 0.519 & 2323.724 & 192.751 & 3900.696 \\
\hline 0.534 & 0.657 & 0.048 & 0.281 & 2741.452 & 775.883 & 4087.274 \\
\hline 0.101 & 0.771 & 0.057 & 0.185 & 5369.277 & 702.136 & 5114.436 \\
\hline 0.021 & 0.830 & 0.035 & 0.238 & 11174.368 & 1103.049 & 6382.696 \\
\hline 0.340 & 0.607 & 0.027 & 0.100 & 1066.975 & 65.204 & 2797.959 \\
\hline 0.568 & 0.680 & 0.027 & -0.024 & 1292.434 & 163.010 & 3096.180 \\
\hline 0.153 & 0.700 & 0.022 & 0.143 & 1901.086 & 99.765 & 3592.483 \\
\hline 0.032 & 0.783 & 0.022 & -0.020 & 12164.833 & 2172.292 & 6513.781 \\
\hline 1.622 & 0.382 & 0.049 & -0.732 & 531.837 & 74.210 & 1802.257 \\
\hline 0.371 & 0.684 & 0.026 & -0.448 & 1180.690 & 88.111 & 3026.470 \\
\hline 0.050 & 0.840 & 0.025 & 0.033 & 8904.315 & 1314.731 & 5948.046 \\
\hline 0.688 & 0.740 & 0.120 & 0.079 & 2169.039 & 596.486 & 3819.059 \\
\hline 0.572 & 0.669 & 0.046 & -0.102 & 1329.256 & 174.138 & 3143.197 \\
\hline 0.134 & 0.825 & 0.043 & 0.198 & 3488.547 & 335.250 & 4474.508 \\
\hline 0.569 & 0.670 & 0.081 & -0.117 & 1428.153 & 201.554 & 3268.392 \\
\hline 0.172 & 0.720 & 0.041 & 0.520 & 1616.495 & 79.407 & 3450.128 \\
\hline 0.154 & 0.881 & 0.079 & 0.577 & 3767.592 & 460.273 & 4602.325 \\
\hline 0.499 & 0.623 & 0.066 & 0.341 & 1061.509 & 94.884 & 2749.708 \\
\hline 0.435 & 0.800 & 0.120 & 0.422 & 2237.741 & 404.411 & 3876.440 \\
\hline 0.279 & 0.691 & 0.058 & 0.246 & 1459.071 & 103.399 & 3260.236 \\
\hline 0.345 & 0.759 & 0.085 & 0.415 & 2214.922 & 313.330 & 3858.429 \\
\hline 0.207 & 0.766 & 0.040 & -0.267 & 3247.710 & 441.292 & 4269.374 \\
\hline 0.177 & 0.789 & 0.019 & -0.328 & 4205.158 & 685.624 & 4696.119 \\
\hline 0.116 & 0.808 & 0.017 & -0.133 & 5312.976 & 788.645 & 5039.188 \\
\hline 0.234 & 0.826 & 0.034 & 0.104 & 4994.212 & 1365.938 & 4988.227 \\
\hline 0.111 & 0.741 & 0.024 & 0.432 & 2622.386 & 146.269 & 4029.449 \\
\hline 0.236 & 0.763 & 0.034 & -0.468 & 5828.965 & 2022.294 & 5172.108 \\
\hline 0.027 & 0.797 & 0.016 & 0.226 & 6521.198 & 313.018 & 5339.429 \\
\hline 0.098 & 0.804 & 0.036 & -0.600 & 6233.578 & 994.313 & 5289.624 \\
\hline 0.033 & 0.824 & 0.015 & -0.048 & 14512.759 & 4097.720 & 6885.371 \\
\hline 0.244 & 0.754 & 0.031 & -0.075 & 2043.501 & 186.271 & 3725.112 \\
\hline 0.353 & 0.724 & 0.051 & -0.159 & 1828.680 & 211.859 & 3598.369 \\
\hline 0.038 & 0.841 & 0.016 & 0.280 & 7907.896 & 721.883 & 5706.860 \\
\hline 0.139 & 0.795 & 0.020 & -0.034 & 4103.638 & 507.191 & 4686.115 \\
\hline 0.383 & 0.766 & 0.033 & -0.300 & 5616.718 & 2990.195 & 5134.026 \\
\hline 0.030 & 0.827 & 0.018 & 0.222 & 6683.235 & 367.211 & 5409.835 \\
\hline 0.584 & 0.722 & 0.030 & -0.357 & 2104.251 & 474.274 & 3693.543 \\
\hline 0.210 & 0.774 & 0.029 & 0.192 & 2800.171 & 319.137 & 4112.346 \\
\hline 0.166 & 0.787 & 0.023 & -0.468 & 6084.808 & 1589.026 & 5220.758 \\
\hline 0.328 & 0.698 & 0.029 & -0.337 & 1484.724 & 126.116 & 3292.473 \\
\hline 0.234 & 0.763 & 0.021 & -0.214 & 2874.888 & 378.544 & 4128.143 \\
\hline 0.210 & 0.759 & 0.027 & -0.132 & 2038.804 & 158.939 & 3765.733 \\
\hline 0.241 & 0.747 & 0.032 & -0.726 & 2812.684 & 370.225 & 4136.815 \\
\hline 0.097 & 0.782 & 0.017 & -0.230 & 4338.079 & 402.176 & 4725.552 \\
\hline 0.132 & 0.795 & 0.023 & -0.269 & 3709.841 & 381.475 & 4557.685 \\
\hline 0.063 & 0.823 & 0.017 & 0.201 & 6973.764 & 858.309 & 5471.972 \\
\hline
\end{tabular}




\begin{tabular}{|c|c|c|c|c|c|c|}
\hline 0.131 & 0.775 & 0.028 & 0.279 & 2866.629 & 209.878 & 4170.777 \\
\hline 0.071 & 0.809 & 0.016 & -0.194 & 5507.915 & 530.392 & 5101.163 \\
\hline 0.041 & 0.831 & 0.022 & -0.203 & 7167.494 & 597.291 & 5512.866 \\
\hline 0.040 & 0.831 & 0.025 & 0.455 & 5643.634 & 314.431 & 5138.543 \\
\hline 0.057 & 0.789 & 0.019 & 0.085 & 3986.615 & 195.605 & 4616.420 \\
\hline 0.063 & 0.790 & 0.055 & 0.182 & 5750.189 & 519.114 & 5140.473 \\
\hline 0.082 & 0.810 & 0.066 & 0.561 & 4633.779 & 400.831 & 4902.588 \\
\hline 0.033 & 0.781 & 0.040 & 0.293 & 10859.277 & 1583.129 & 6300.003 \\
\hline 0.338 & 0.820 & 0.110 & 0.362 & 4000.493 & 1162.934 & 4735.177 \\
\hline 0.062 & 0.900 & 0.120 & 0.418 & 6995.579 & 856.602 & 5630.479 \\
\hline 0.719 & 0.647 & 0.048 & -0.274 & 1586.324 & 318.402 & 3387.084 \\
\hline 0.062 & 0.837 & 0.044 & 0.463 & 5616.718 & 484.897 & 5165.859 \\
\hline 0.362 & 0.694 & 0.059 & 0.614 & 1631.527 & 169.951 & 3415.588 \\
\hline 0.310 & 0.692 & 0.047 & 0.429 & 1745.626 & 168.388 & 3466.967 \\
\hline 0.252 & 0.806 & 0.052 & 0.129 & 4103.638 & 922.166 & 4676.012 \\
\hline 0.131 & 0.802 & 0.058 & 0.082 & 5802.813 & 1112.868 & 5238.417 \\
\hline 0.055 & 0.781 & 0.048 & 0.301 & 4767.469 & 287.354 & 4861.138 \\
\hline 0.014 & 0.838 & 0.026 & 0.160 & 10871.226 & 667.098 & 6322.320 \\
\hline 0.202 & 0.823 & 0.064 & 0.295 & 2323.724 & 203.460 & 4027.528 \\
\hline 0.225 & 0.791 & 0.073 & 0.223 & 3533.418 & 580.836 & 4486.816 \\
\hline 0.174 & 0.718 & 0.077 & 0.478 & 3302.012 & 384.988 & 4257.177 \\
\hline 0.222 & 0.740 & 0.081 & 0.490 & 2155.211 & 190.006 & 3816.695 \\
\hline 0.111 & 0.832 & 0.078 & 0.243 & 3842.614 & 348.900 & 4597.948 \\
\hline 0.129 & 0.793 & 0.063 & -0.031 & 2605.195 & 167.402 & 4103.463 \\
\hline 1.036 & 0.475 & 0.066 & -0.275 & 684.353 & 79.434 & 2208.206 \\
\hline 0.572 & 0.589 & 0.024 & -0.360 & 967.882 & 89.619 & 2686.881 \\
\hline 0.275 & 0.807 & 0.084 & 0.339 & 2399.697 & 297.967 & 3964.046 \\
\hline 0.678 & 0.614 & 0.052 & -0.194 & 1045.085 & 124.698 & 2796.020 \\
\hline 0.108 & 0.828 & 0.067 & 0.681 & 5535.288 & 814.013 & 5114.436 \\
\hline 0.178 & 0.757 & 0.041 & 0.010 & 3860.348 & 564.929 & 4546.212 \\
\hline
\end{tabular}


Page 96 of 203 


\begin{tabular}{|c|c|c|c|c|}
\hline Error $2 \sigma$ & FinalAge ${ }^{207} \mathrm{~Pb}$ & Error $2 \sigma$ & FinalAge $207 \mathrm{~Pb}$ corr & Error $2 \sigma$ \\
\hline 29.716 & 1473.566 & 39.912 & 1034.455 & 16.528 \\
\hline 29.917 & 1399.849 & 34.681 & 1026.331 & 17.090 \\
\hline 30.588 & 1335.933 & 34.215 & 1041.105 & 17.142 \\
\hline 42.195 & 1340.429 & 43.591 & 1027.880 & 17.268 \\
\hline 31.429 & 1431.882 & 31.714 & 1038.571 & 21.475 \\
\hline 33.218 & 1382.484 & 34.562 & 1020.531 & 14.884 \\
\hline 31.079 & 1412.744 & 36.346 & 1038.543 & 14.328 \\
\hline 36.096 & 1360.495 & 39.095 & 1062.308 & 16.624 \\
\hline 24.117 & 1442.411 & 31.771 & 1035.156 & 13.687 \\
\hline 26.728 & 1433.994 & 30.139 & 1042.940 & 12.014 \\
\hline 28.104 & 1333.681 & 37.306 & 1020.896 & 16.076 \\
\hline 30.841 & 1382.484 & 37.704 & 1039.682 & 16.032 \\
\hline 32.411 & 1423.406 & 42.750 & 1060.956 & 17.168 \\
\hline 34.249 & 1448.694 & 36.575 & 1026.375 & 14.853 \\
\hline 31.711 & 1373.727 & 31.364 & 1008.038 & 13.742 \\
\hline 33.934 & 1408.458 & 42.633 & 1020.787 & 17.739 \\
\hline 28.305 & 1463.252 & 39.849 & 1022.728 & 14.888 \\
\hline 40.659 & 1461.181 & 50.990 & 1052.557 & 14.014 \\
\hline 47.552 & 1384.666 & 64.440 & 1052.505 & 16.543 \\
\hline 30.583 & 1378.112 & 34.531 & 1065.817 & 14.329 \\
\hline 36.509 & 1487.888 & 43.197 & 1523.232 & 30.815 \\
\hline 47.866 & 1573.001 & 54.966 & 1504.857 & 27.610 \\
\hline 53.397 & 1465.320 & 47.834 & 1543.420 & 30.956 \\
\hline 52.218 & 1512.128 & 41.736 & 1561.021 & 26.962 \\
\hline 60.178 & 1539.924 & 53.156 & 1543.089 & 32.009 \\
\hline 52.362 & 1539.924 & 38.659 & 1518.786 & 37.227 \\
\hline 68.682 & 1557.526 & 62.947 & 1531.591 & 31.625 \\
\hline 40.586 & 1483.809 & 36.775 & 1559.046 & 28.014 \\
\hline 60.178 & 1530.055 & 45.049 & 1564.970 & 28.635 \\
\hline 41.927 & 1510.122 & 38.515 & 1558.429 & 30.738 \\
\hline 52.003 & 1514.130 & 48.170 & 1560.846 & 22.759 \\
\hline 40.051 & 1547.772 & 45.143 & 1554.544 & 22.094 \\
\hline 47.347 & 1559.469 & 48.431 & 1552.940 & 23.770 \\
\hline 47.670 & 1555.580 & 53.251 & 1538.939 & 34.695 \\
\hline 51.442 & 1594.022 & 48.598 & 1543.176 & 44.363 \\
\hline 61.258 & 1498.035 & 54.474 & 1555.604 & 27.247 \\
\hline 55.880 & 1574.924 & 53.360 & 1562.015 & 36.320 \\
\hline 36.583 & 1481.766 & 31.969 & 1546.469 & 22.445 \\
\hline 66.841 & 1522.114 & 54.648 & 1503.189 & 24.988 \\
\hline 56.961 & 1534.010 & 51.509 & 1516.537 & 30.326 \\
\hline 605.964 & 4710.033 & 580.221 & 392.959 & 200.363 \\
\hline 467.479 & 4689.023 & 758.518 & 245.631 & 157.484 \\
\hline 682.249 & 4810.418 & 780.068 & 465.316 & 436.577 \\
\hline 811.745 & 4939.422 & 975.688 & 330.614 & 1083.929 \\
\hline 824.561 & 4965.520 & 258.809 & 835.558 & 532.600 \\
\hline 1422.574 & 4949.923 & 564.146 & 848.010 & 1002.931 \\
\hline 997.645 & 4878.312 & 339.470 & 1570.974 & 566.641 \\
\hline
\end{tabular}




\begin{tabular}{|c|c|c|c|c|}
\hline 940.596 & 4970.679 & 522.281 & 67.214 & 475.481 \\
\hline 517.652 & 4891.124 & 493.485 & 160.279 & 200.902 \\
\hline 357.737 & 4755.129 & 340.606 & 363.478 & 130.747 \\
\hline 549.932 & 4829.625 & 540.918 & 208.059 & 177.380 \\
\hline 306.411 & 4663.371 & 432.828 & 438.751 & 141.606 \\
\hline 712.721 & 4829.625 & 643.950 & 440.561 & 378.961 \\
\hline 443.787 & 4747.041 & 328.538 & 388.232 & 128.974 \\
\hline 528.964 & 4710.033 & 750.875 & 470.812 & 290.457 \\
\hline 547.590 & 4728.664 & 493.838 & 254.143 & 125.898 \\
\hline 3901.872 & 4761.163 & 426.174 & 2816.309 & 3305.071 \\
\hline 329.067 & 4367.791 & 392.701 & 382.578 & 71.414 \\
\hline 1434.235 & 4065.230 & 732.474 & 533.375 & 254.291 \\
\hline 323.493 & 4639.420 & 268.338 & 376.535 & 75.876 \\
\hline 345.275 & 4553.243 & 242.742 & 357.428 & 58.120 \\
\hline 383.862 & 4293.266 & 530.442 & 524.253 & 101.988 \\
\hline 203.096 & 4435.883 & 489.423 & 375.609 & 88.821 \\
\hline 1161.656 & 4775.137 & 456.350 & 522.825 & 277.438 \\
\hline 280.600 & 4133.909 & 533.408 & 402.866 & 69.883 \\
\hline 266.494 & 4174.756 & 506.560 & 299.260 & 52.925 \\
\hline 491.481 & 4854.190 & 470.786 & 290.856 & 234.574 \\
\hline 375.365 & 4608.308 & 587.685 & 284.107 & 111.695 \\
\hline 1394.725 & 4567.221 & 358.070 & 547.612 & 208.941 \\
\hline 298.974 & 4536.749 & 526.322 & 368.427 & 109.309 \\
\hline 262.181 & 4861.659 & 329.604 & 487.491 & 247.424 \\
\hline 790.920 & 4848.561 & 637.969 & 869.667 & 624.755 \\
\hline 532.589 & 4887.476 & 575.733 & 268.635 & 339.939 \\
\hline 440.212 & 2507.554 & 471.116 & 351.289 & 60.450 \\
\hline 487.058 & 4775.137 & 548.942 & 457.427 & 264.417 \\
\hline 1292.227 & 4804.602 & 560.646 & 1979.795 & 847.913 \\
\hline 390.450 & 4707.946 & 389.482 & 287.583 & 103.734 \\
\hline 1355.113 & 4902.008 & 602.655 & 390.045 & 522.286 \\
\hline 954.198 & 4921.737 & 738.260 & 513.290 & 854.391 \\
\hline 669.307 & 4844.795 & 549.673 & 736.457 & 489.066 \\
\hline 981.716 & 4923.516 & 577.791 & 196.824 & 388.720 \\
\hline 961.782 & 4867.233 & 632.108 & 420.946 & 450.886 \\
\hline 477.719 & 4652.537 & 308.766 & 269.914 & 68.248 \\
\hline 1097.429 & 4541.482 & 621.310 & 565.516 & 220.936 \\
\hline 433.825 & 4918.171 & 505.376 & 130.615 & 231.936 \\
\hline 296.442 & 4953.404 & 369.386 & 94.254 & 242.520 \\
\hline 231.931 & 4846.679 & 223.496 & 452.590 & 149.723 \\
\hline 945.657 & 4970.679 & 192.104 & 2113.084 & 599.165 \\
\hline 912.065 & 5047.387 & 144.376 & -7773.769 & 3123.516 \\
\hline 1163.797 & 4921.737 & 166.109 & 465.880 & 220.223 \\
\hline 420.664 & 4955.142 & 151.256 & 245.230 & 191.232 \\
\hline 791.178 & 4788.968 & 335.031 & 473.007 & 188.582 \\
\hline 1200.005 & 4949.923 & 430.692 & 897.541 & 765.974 \\
\hline 177.197 & 4829.625 & 772.740 & 25.991 & 35.883 \\
\hline 758.689 & 4757.143 & 386.977 & 985.294 & 334.588 \\
\hline 356.283 & 4736.863 & 370.594 & 306.856 & 113.107 \\
\hline
\end{tabular}




\begin{tabular}{|c|c|c|c|c|}
\hline 1667.216 & 4738.905 & 558.706 & 323.205 & 223.438 \\
\hline 487.418 & 4767.170 & 358.534 & 222.103 & 92.374 \\
\hline 959.198 & 4794.852 & 275.114 & 703.929 & 238.191 \\
\hline 325.058 & 4869.086 & 397.863 & 284.823 & 214.628 \\
\hline 1263.339 & 4639.420 & 338.953 & 939.459 & 312.357 \\
\hline 935.975 & 4869.086 & 359.971 & 1596.406 & 521.135 \\
\hline 595.401 & 4974.107 & 209.752 & 1850.412 & 1221.136 \\
\hline 182.352 & 4524.844 & 201.270 & 353.447 & 43.587 \\
\hline 369.693 & 4689.023 & 186.182 & 306.030 & 60.963 \\
\hline 182.851 & 4730.719 & 148.680 & 435.922 & 63.696 \\
\hline 1174.616 & 4891.124 & 137.426 & 0.000 & 1858.966 \\
\hline 441.369 & 3839.697 & 492.527 & 319.368 & 54.613 \\
\hline 275.134 & 4697.466 & 178.559 & 268.039 & 46.358 \\
\hline 903.285 & 4991.118 & 148.545 & 0.000 & 850.375 \\
\hline 1363.950 & 4810.418 & 780.068 & 375.003 & 380.281 \\
\hline 461.797 & 4665.528 & 320.799 & 337.926 & 92.534 \\
\hline 475.071 & 4965.520 & 258.809 & 101.911 & 251.876 \\
\hline 585.587 & 4667.681 & 564.302 & 366.477 & 162.029 \\
\hline 214.887 & 4771.159 & 271.691 & 303.261 & 92.735 \\
\hline 500.253 & 5058.683 & 453.616 & -518.623 & 571.843 \\
\hline 274.971 & 4562.578 & 483.355 & 327.836 & 95.648 \\
\hline 723.021 & 4921.737 & 738.260 & 151.053 & 397.099 \\
\hline 301.366 & 4712.115 & 395.518 & 330.543 & 116.271 \\
\hline 556.249 & 4846.679 & 542.777 & 312.718 & 273.158 \\
\hline 511.031 & 4859.795 & 253.775 & 526.503 & 209.876 \\
\hline 836.932 & 4902.008 & 118.046 & 618.570 & 169.432 \\
\hline 745.232 & 4935.904 & 103.849 & 763.448 & 203.842 \\
\hline 1367.144 & 4967.242 & 204.463 & 261.000 & 325.582 \\
\hline 194.097 & 4812.351 & 155.866 & 491.381 & 97.800 \\
\hline 1819.816 & 4854.190 & 216.307 & 2329.268 & 902.691 \\
\hline 251.333 & 4916.385 & 98.698 & 2274.218 & 229.987 \\
\hline 959.108 & 4928.839 & 220.694 & 1586.692 & 460.118 \\
\hline 1877.828 & 4963.796 & 90.360 & \#VALUE! & \#VALUE! \\
\hline 380.313 & 4837.232 & 198.878 & 296.440 & 95.726 \\
\hline 556.890 & 4779.104 & 336.650 & 344.135 & 135.272 \\
\hline 560.310 & 4992.807 & 94.988 & 1167.503 & 314.074 \\
\hline 656.056 & 4912.805 & 123.593 & 515.254 & 155.283 \\
\hline 2863.207 & 4859.795 & 209.365 & 1968.621 & 1082.343 \\
\hline 290.284 & 4968.962 & 108.152 & 994.687 & 254.744 \\
\hline 888.447 & 4775.137 & 198.413 & 423.259 & 129.745 \\
\hline 466.649 & 4874.629 & 182.641 & 363.894 & 131.128 \\
\hline 1412.676 & 4898.390 & 143.155 & 1994.055 & 572.291 \\
\hline 321.217 & 4726.607 & 196.378 & 322.072 & 65.218 \\
\hline 554.742 & 4854.190 & 133.602 & 443.899 & 110.438 \\
\hline 369.004 & 4846.679 & 172.412 & 278.055 & 83.652 \\
\hline 658.455 & 4823.892 & 206.646 & 516.189 & 149.140 \\
\hline 499.818 & 4889.301 & 106.289 & 755.601 & 144.969 \\
\hline 507.560 & 4912.805 & 142.131 & 412.261 & 149.142 \\
\hline 652.621 & 4962.070 & 102.497 & 1557.591 & 303.804 \\
\hline
\end{tabular}




$\begin{array}{ccccc}320.829 & 4876.472 & 176.182 & 372.455 & 126.852 \\ 506.738 & 4937.664 & 97.655 & 842.833 & 187.630 \\ 485.715 & 4975.818 & 131.730 & 1227.460 & 338.128 \\ 262.338 & 4975.818 & 149.694 & 281.068 & 285.428 \\ 247.397 & 4902.008 & 118.046 & 547.157 & 132.688 \\ & & & & \\ 523.870 & 4903.814 & 341.405 & 1521.680 & 535.476 \\ 434.907 & 4939.422 & 402.471 & 465.050 & 525.907 \\ 930.972 & 4887.476 & 250.319 & \text { \#VALUE! } & \text { \#VALUE! } \\ 1398.005 & 4956.877 & 664.947 & 200.911 & 747.813 \\ 596.168 & 5088.870 & 678.516 & -9576.750 & 10702.179 \\ 837.397 & 4617.270 & 342.549 & 471.613 & 137.189 \\ 449.205 & 4986.038 & 262.110 & 86.062 & 498.014 \\ 318.298 & 4718.345 & 401.127 & 372.530 & 135.895 \\ 318.395 & 4714.195 & 320.184 & 411.601 & 118.431 \\ 991.881 & 4932.376 & 318.218 & 385.601 & 366.103 \\ 1059.795 & 4925.292 & 356.193 & 1236.119 & 615.626 \\ 408.499 & 4887.476 & 300.383 & 978.164 & 371.950 \\ 363.064 & 4987.733 & 154.751 & -286.523 & 1020.538 \\ 404.308 & 4962.070 & 385.872 & 57.655 & 226.222 \\ 766.042 & 4905.617 & 452.731 & 407.501 & 411.383 \\ 444.000 & 4767.170 & 511.242 & 874.842 & 375.637 \\ 446.344 & 4810.418 & 526.546 & 371.627 & 248.348 \\ 491.920 & 4977.527 & 466.643 & 49.873 & 516.650 \\ 308.310 & 4909.216 & 390.013 & 227.287 & 247.916 \\ 452.965 & 4165.438 & 578.777 & 334.706 & 68.532 \\ 307.658 & 4481.095 & 182.591 & 340.244 & 43.587 \\ 579.110 & 4934.141 & 513.591 & 135.552 & 303.410 \\ 437.473 & 4541.482 & 384.621 & 335.078 & 80.925 \\ 534.843 & 4970.679 & 402.217 & 340.959 & 704.188 \\ 679.319 & 4842.908 & 262.298 & 820.106 & 268.033\end{array}$




\begin{tabular}{|c|c|c|c|c|c|c|}
\hline Sample & ${ }^{207} \mathrm{~Pb} /{ }^{235} \mathrm{U}$ & Error $2 \sigma$ & ${ }^{206} \mathrm{~Pb} /{ }^{238} \mathrm{U}$ & Error $2 \sigma$ & rrelation_6_3 & ${ }^{8} \mathrm{U} /{ }^{206} \mathrm{~Pb}$ \\
\hline R_R19_1 & 0.680 & 0.120 & 0.088 & 0.005 & 0.244 & 11.429 \\
\hline R_R19_2 & 0.970 & 0.150 & 0.094 & 0.005 & -0.098 & 10.616 \\
\hline R_R19_3 & 0.760 & 0.120 & 0.087 & 0.005 & 0.216 & 11.494 \\
\hline R_R19_4 & 0.880 & 0.100 & 0.079 & 0.003 & 0.261 & 12.674 \\
\hline R_R19_5 & 1.300 & 1.000 & 0.084 & 0.005 & 0.005 & 11.862 \\
\hline R_R19_6 & 0.680 & 0.130 & 0.080 & 0.004 & 0.937 & 12.563 \\
\hline R_R19_7 & 1.050 & 0.580 & 0.087 & 0.006 & -0.036 & 11.547 \\
\hline R_R19_8 & 1.200 & 1.100 & 0.087 & 0.013 & 0.428 & 11.494 \\
\hline R_R19_9 & 0.600 & 0.110 & 0.086 & 0.004 & 0.672 & 11.574 \\
\hline R_R19_10 & 0.672 & 0.094 & 0.077 & 0.004 & 0.247 & 13.072 \\
\hline R_R19_11 & 6.900 & 6.300 & 0.084 & 0.011 & 0.889 & 11.905 \\
\hline R_R19_12 & 0.649 & 0.089 & 0.076 & 0.003 & -0.043 & 13.245 \\
\hline R_R19_13 & 1.230 & 0.680 & 0.079 & 0.003 & 0.219 & 12.723 \\
\hline R_R19_14 & 0.940 & 0.360 & 0.085 & 0.004 & 0.661 & 11.792 \\
\hline R_R19_16 & 0.614 & 0.085 & 0.084 & 0.002 & 0.030 & 11.976 \\
\hline R_R19_18 & 1.310 & 0.950 & 0.084 & 0.009 & 0.067 & 11.905 \\
\hline X73_1 & 100.000 & 17.000 & 0.850 & 0.140 & 0.968 & 1.176 \\
\hline$x 73 \_2$ & 168.000 & 10.000 & 1.480 & 0.100 & 0.949 & 0.676 \\
\hline$x 73 \_3$ & 121.500 & 5.800 & 1.090 & 0.049 & 0.646 & 0.917 \\
\hline$x 73 \_4$ & 112.000 & 5.300 & 0.974 & 0.045 & 0.908 & 1.027 \\
\hline$x 73 \_5$ & 224.900 & 9.700 & 1.946 & 0.098 & 0.893 & 0.514 \\
\hline$x 73 \_6$ & 253.000 & 22.000 & 2.320 & 0.200 & 0.073 & 0.431 \\
\hline$x 73 \_7$ & 3.720 & 0.700 & 0.040 & 0.006 & 0.916 & 24.752 \\
\hline$x 73 \_8$ & 64.500 & 3.800 & 0.575 & 0.029 & 0.889 & 1.739 \\
\hline X73_9 & 204.000 & 30.000 & 1.860 & 0.280 & 0.979 & 0.538 \\
\hline X73_10 & 110.900 & 4.000 & 0.959 & 0.030 & 0.740 & 1.043 \\
\hline X73_11 & 291.000 & 13.000 & 2.520 & 0.110 & 0.861 & 0.397 \\
\hline X73_12 & 117.300 & 5.200 & 1.041 & 0.042 & 0.943 & 0.961 \\
\hline$x 73 \_13$ & 249.000 & 14.000 & 2.210 & 0.130 & 0.971 & 0.452 \\
\hline X73_14 & 136.500 & 5.500 & 1.196 & 0.047 & 0.950 & 0.836 \\
\hline$x 73 \_15$ & 336.000 & 11.000 & 2.937 & 0.096 & 0.869 & 0.340 \\
\hline$x 73 \_16$ & 300.000 & 17.000 & 2.600 & 0.130 & 0.919 & 0.385 \\
\hline$x 73 \_17$ & 176.000 & 14.000 & 1.560 & 0.120 & 0.972 & 0.641 \\
\hline X73_18 & 167.000 & 8.900 & 1.418 & 0.084 & 0.916 & 0.705 \\
\hline X73_19 & 192.500 & 9.500 & 1.648 & 0.081 & 0.866 & 0.607 \\
\hline$X 73 \_20$ & 209.000 & 12.000 & 1.828 & 0.095 & 0.151 & 0.547 \\
\hline X73_21 & 219.000 & 12.000 & 1.902 & 0.094 & 0.951 & 0.526 \\
\hline$x 73 \_22$ & 271.000 & 11.000 & 2.390 & 0.110 & 0.546 & 0.418 \\
\hline$x 73 \_23$ & 478.000 & 42.000 & 4.220 & 0.370 & 0.975 & 0.237 \\
\hline$x 73 \_24$ & 271.000 & 11.000 & 2.260 & 0.200 & 0.761 & 0.442 \\
\hline X73_25 & 244.000 & 24.000 & 2.140 & 0.220 & 1.000 & 0.467 \\
\hline$X 73 \_26$ & 190.000 & 11.000 & 1.720 & 0.120 & 0.767 & 0.581 \\
\hline$x 73 \_27$ & 188.000 & 16.000 & 1.620 & 0.110 & 0.495 & 0.617 \\
\hline$X 73 \_28$ & 170.400 & 6.200 & 1.485 & 0.060 & 0.862 & 0.673 \\
\hline$x 73 \_29$ & 154.600 & 9.000 & 1.387 & 0.053 & 0.625 & 0.721 \\
\hline X73_30 & 73.700 & 7.000 & 0.627 & 0.056 & 0.920 & 1.595 \\
\hline X73_31 & 250.000 & 17.000 & 2.220 & 0.150 & 0.926 & 0.450 \\
\hline X73_32 & 155.000 & 12.000 & 1.810 & 0.670 & 0.583 & 0.552 \\
\hline
\end{tabular}




\begin{tabular}{|c|c|c|c|c|c|c|}
\hline X73_33 & 289.000 & 19.000 & 2.510 & 0.160 & 0.935 & 0.398 \\
\hline X73_ 34 & 180.000 & 10.000 & 1.571 & 0.087 & 0.856 & 0.637 \\
\hline$x 73 \_35$ & 183.000 & 16.000 & 1.550 & 0.140 & 0.953 & 0.645 \\
\hline$x 73 \_36$ & 374.000 & 16.000 & 3.240 & 0.150 & 0.909 & 0.309 \\
\hline X73__37 & 296.000 & 15.000 & 2.560 & 0.130 & 0.857 & 0.391 \\
\hline$X 73 \_38$ & 207.500 & 8.800 & 1.858 & 0.079 & 0.432 & 0.538 \\
\hline X73_39 & 76.200 & 5.300 & 0.701 & 0.053 & 0.882 & 1.427 \\
\hline$x 73 \_40$ & 9.500 & 1.300 & 0.117 & 0.013 & 0.757 & 8.547 \\
\hline$X 73 \_41$ & 37.400 & 7.600 & 0.364 & 0.061 & 0.961 & 2.747 \\
\hline$X 73 \_42$ & 23.600 & 2.900 & 0.223 & 0.021 & 0.782 & 4.484 \\
\hline$X 73 \_43$ & 13.400 & 1.100 & 0.142 & 0.008 & 0.828 & 7.057 \\
\hline$X 73 \_44$ & 95.800 & 3.300 & 0.877 & 0.030 & 0.672 & 1.140 \\
\hline$X 73 \_45$ & 99.000 & 9.000 & 0.863 & 0.075 & 0.963 & 1.159 \\
\hline$X 73 \_46$ & 167.000 & 16.000 & 1.520 & 0.150 & 0.965 & 0.658 \\
\hline$X 73 \_47$ & 104.300 & 4.600 & 0.894 & 0.038 & 0.901 & 1.119 \\
\hline$X 73 \_48$ & 103.500 & 4.700 & 0.924 & 0.051 & 0.637 & 1.082 \\
\hline$x 73 \_49$ & 456.000 & 32.000 & 3.910 & 0.260 & 0.909 & 0.256 \\
\hline$X 73 \_50$ & 354.000 & 15.000 & 3.030 & 0.150 & 0.931 & 0.330 \\
\hline X79_1 & 35.400 & 2.900 & 0.322 & 0.027 & 0.973 & 3.106 \\
\hline X79_2 & 272.000 & 22.000 & 2.320 & 0.190 & 0.987 & 0.431 \\
\hline X79_3 & 37.100 & 3.900 & 0.345 & 0.036 & 0.950 & 2.899 \\
\hline X79_4 & 23.900 & 2.900 & 0.222 & 0.024 & 0.963 & 4.505 \\
\hline X79_5 & 51.500 & 3.700 & 0.463 & 0.032 & 0.761 & 2.160 \\
\hline X79_6 & 82.300 & 4.600 & 0.726 & 0.038 & 0.152 & 1.377 \\
\hline X79_7 & 72.900 & 4.600 & 0.678 & 0.055 & 0.349 & 1.475 \\
\hline X79_8 & 125.800 & 6.100 & 1.116 & 0.052 & 0.769 & 0.896 \\
\hline X79_9 & 98.300 & 9.100 & 0.872 & 0.080 & 0.938 & 1.147 \\
\hline X79_10 & 183.000 & 6.100 & 1.589 & 0.052 & 0.934 & 0.629 \\
\hline X79_11 & 303.000 & 19.000 & 2.570 & 0.150 & 0.987 & 0.389 \\
\hline X79_12 & 123.800 & 9.500 & 1.079 & 0.083 & 0.930 & 0.927 \\
\hline$X 79 \_13$ & 193.000 & 11.000 & 1.703 & 0.092 & 0.956 & 0.587 \\
\hline X79_14 & 140.000 & 8.000 & 1.217 & 0.070 & 0.913 & 0.822 \\
\hline$X 79 \_15$ & 132.900 & 5.600 & 1.172 & 0.047 & 0.855 & 0.853 \\
\hline$X 79 \_16$ & 135.300 & 6.800 & 1.177 & 0.066 & 0.872 & 0.850 \\
\hline X79_17 & 192.000 & 11.000 & 1.685 & 0.098 & 0.145 & 0.593 \\
\hline$X 79 \_18$ & 130.300 & 8.100 & 1.113 & 0.070 & 0.896 & 0.898 \\
\hline X79_19 & 81.700 & 3.300 & 0.701 & 0.030 & 0.755 & 1.427 \\
\hline$X 79 \_20$ & 142.400 & 7.600 & 1.227 & 0.069 & 0.904 & 0.815 \\
\hline X79_21 & 124.500 & 8.400 & 1.108 & 0.072 & 0.899 & 0.903 \\
\hline$x 79 \_22$ & 152.700 & 8.600 & 1.328 & 0.065 & 0.930 & 0.753 \\
\hline$x 79 \_23$ & 236.200 & 8.400 & 2.038 & 0.081 & 0.807 & 0.491 \\
\hline$x 79 \_24$ & 45.900 & 7.500 & 0.422 & 0.073 & 0.982 & 2.370 \\
\hline$x 79 \_25$ & 143.600 & 5.300 & 1.226 & 0.052 & 0.652 & 0.816 \\
\hline$x 79 \_26$ & 148.100 & 5.800 & 1.295 & 0.055 & 0.885 & 0.772 \\
\hline$X 79 \_27$ & 199.000 & 14.000 & 1.740 & 0.120 & 0.932 & 0.575 \\
\hline$X 79 \_28$ & 461.000 & 47.000 & 3.840 & 0.370 & 0.986 & 0.260 \\
\hline$X 79 \_29$ & 676.000 & 56.000 & 5.730 & 0.480 & 0.992 & 0.175 \\
\hline$X 79 \_30$ & 117.000 & 13.000 & 1.090 & 0.130 & 0.293 & 0.917 \\
\hline X79_31 & 143.000 & 15.000 & 1.210 & 0.120 & 0.925 & 0.826 \\
\hline
\end{tabular}




\begin{tabular}{|c|c|c|c|c|c|c|}
\hline$x 79 \_32$ & 161.300 & 8.100 & 1.490 & 0.120 & 0.240 & 0.671 \\
\hline$X 79 \_33$ & 180.000 & 40.000 & 1.400 & 0.290 & 0.993 & 0.714 \\
\hline$x 79 \_34$ & 325.000 & 15.000 & 2.770 & 0.140 & 0.922 & 0.361 \\
\hline$X 79 \_35$ & 170.000 & 12.000 & 1.450 & 0.100 & 0.984 & 0.690 \\
\hline X79__36 & 174.700 & 7.500 & 1.482 & 0.063 & -0.097 & 0.675 \\
\hline X79__37 & 152.000 & 31.000 & 1.320 & 0.280 & 0.994 & 0.758 \\
\hline$X 79 \_38$ & 170.600 & 9.300 & 1.497 & 0.049 & 0.987 & 0.668 \\
\hline X79_39 & 130.800 & 9.400 & 1.088 & 0.092 & 0.439 & 0.919 \\
\hline$X 79 \_40$ & 180.000 & 14.000 & 1.540 & 0.120 & 0.991 & 0.649 \\
\hline$x 79 \_41$ & 685.000 & 47.000 & 5.900 & 0.420 & 0.990 & 0.169 \\
\hline$X 79 \_42$ & 448.000 & 50.000 & 3.700 & 0.380 & 0.982 & 0.270 \\
\hline$X 79 \_43$ & 134.100 & 6.200 & 1.172 & 0.081 & 0.307 & 0.853 \\
\hline$X 79 \_44$ & 337.000 & 26.000 & 2.820 & 0.200 & 0.964 & 0.355 \\
\hline$X 79 \_45$ & 119.000 & 15.000 & 1.050 & 0.130 & 0.995 & 0.952 \\
\hline$X 79 \_46$ & 81.300 & 3.100 & 0.719 & 0.031 & 0.653 & 1.391 \\
\hline$x 79 \_47$ & 133.700 & 6.300 & 1.143 & 0.062 & 0.866 & 0.875 \\
\hline$x 79 \_48$ & 315.000 & 24.000 & 2.810 & 0.210 & 0.961 & 0.356 \\
\hline$X 79 \_49$ & 105.000 & 14.000 & 0.920 & 0.110 & 0.978 & 1.087 \\
\hline$X 79 \_50$ & 182.000 & 8.100 & 1.547 & 0.077 & 0.925 & 0.646 \\
\hline$X 79 \_51$ & 104.300 & 4.600 & 0.933 & 0.045 & 0.863 & 1.072 \\
\hline$X 79 \_52$ & 81.600 & 6.700 & 0.727 & 0.061 & 0.963 & 1.376 \\
\hline$X 79 \_53$ & 364.000 & 25.000 & 3.110 & 0.200 & 0.943 & 0.322 \\
\hline$X 79 \_54$ & 994.000 & 59.000 & 8.730 & 0.560 & 0.951 & 0.115 \\
\hline$X 79 \_55$ & 880.000 & 54.000 & 7.360 & 0.460 & 0.949 & 0.136 \\
\hline$X 79 \_56$ & 417.000 & 21.000 & 3.660 & 0.190 & 0.935 & 0.273 \\
\hline$X 79 \_57$ & 484.000 & 36.000 & 4.060 & 0.280 & 0.965 & 0.246 \\
\hline$X 79 \_58$ & 215.000 & 15.000 & 1.850 & 0.120 & 0.931 & 0.541 \\
\hline X79_59 & 215.000 & 11.000 & 1.880 & 0.100 & 0.780 & 0.532 \\
\hline$X 128 \_1$ & 65.000 & 14.000 & 0.570 & 0.110 & 0.983 & 1.754 \\
\hline$X 128 \_2$ & 29.700 & 6.600 & 0.263 & 0.057 & 0.994 & 3.802 \\
\hline$x 128 \_3$ & 131.000 & 34.000 & 1.180 & 0.300 & 0.994 & 0.847 \\
\hline$X 128 \_4$ & 88.800 & 6.700 & 0.778 & 0.056 & 0.932 & 1.285 \\
\hline$X 128 \_5$ & 405.000 & 58.000 & 3.720 & 0.530 & 0.066 & 0.269 \\
\hline$x 128 \_6$ & 7.550 & 0.950 & 0.098 & 0.009 & 0.985 & 10.194 \\
\hline$x 128 \_7$ & 5.600 & 0.570 & 0.058 & 0.005 & 0.443 & 17.271 \\
\hline$X 128 \_8$ & 16.500 & 1.200 & 0.152 & 0.011 & 0.544 & 6.579 \\
\hline$X 128 \_9$ & 9.400 & 2.000 & 0.083 & 0.018 & 0.763 & 12.048 \\
\hline$X 128 \_10$ & 2.580 & 0.390 & 0.040 & 0.003 & 0.912 & 25.126 \\
\hline X128_11 & 6.490 & 0.640 & 0.083 & 0.008 & -0.079 & 12.107 \\
\hline $\mathrm{X} 128 \_12$ & 17.100 & 1.400 & 0.220 & 0.120 & -0.016 & 4.545 \\
\hline X128_13 & 14.900 & 1.800 & 0.157 & 0.016 & 0.920 & 6.369 \\
\hline X128_14 & 14.700 & 2.000 & 0.148 & 0.027 & 0.406 & 6.757 \\
\hline X128_15 & 104.000 & 36.000 & 0.800 & 0.260 & 0.990 & 1.250 \\
\hline$x 128 \_16$ & 9.300 & 1.100 & 0.093 & 0.011 & 0.919 & 10.753 \\
\hline$X 128 \_17$ & 11.000 & 1.200 & 0.094 & 0.011 & 0.421 & 10.638 \\
\hline X128_18 & 46.400 & 5.400 & 0.432 & 0.056 & 0.622 & 2.315 \\
\hline X128_19 & 5.730 & 0.340 & 0.067 & 0.003 & 0.701 & 14.925 \\
\hline$X 128 \_20$ & 3.770 & 0.280 & 0.043 & 0.001 & -0.116 & 23.529 \\
\hline$X 128 \_21$ & 8.320 & 0.860 & 0.075 & 0.007 & 0.471 & 13.263 \\
\hline
\end{tabular}




\begin{tabular}{|c|c|c|c|c|c|c|}
\hline$x 128 \_22$ & 10.210 & 0.920 & 0.106 & 0.007 & 0.346 & 9.425 \\
\hline$X 128 \_23$ & 9.390 & 0.440 & 0.092 & 0.004 & 0.768 & 10.881 \\
\hline$X 128 \_24$ & 12.560 & 0.720 & 0.124 & 0.007 & 0.800 & 8.045 \\
\hline$X 128 \_25$ & 15.200 & 1.100 & 0.151 & 0.009 & 0.913 & 6.636 \\
\hline$X 128 \_26$ & 38.900 & 3.600 & 0.360 & 0.034 & 0.923 & 2.778 \\
\hline$X 128 \_27$ & 17.220 & 0.680 & 0.171 & 0.005 & -0.030 & 5.851 \\
\hline$X 128 \_28$ & 14.170 & 0.840 & 0.150 & 0.007 & 0.476 & 6.662 \\
\hline$X 128 \_29$ & 5.220 & 0.440 & 0.053 & 0.004 & 0.589 & 18.727 \\
\hline X128_30 & 106.000 & 24.000 & 0.950 & 0.210 & 0.992 & 1.053 \\
\hline X128_31 & 3.670 & 0.370 & 0.040 & 0.004 & 0.795 & 25.063 \\
\hline X128_32 & 9.470 & 0.590 & 0.095 & 0.005 & 0.770 & 10.549 \\
\hline X128_33 & 22.400 & 2.500 & 0.211 & 0.019 & 0.791 & 4.739 \\
\hline$X 128 \_34$ & 13.000 & 1.800 & 0.119 & 0.017 & 0.968 & 8.403 \\
\hline$X 128 \_35$ & 7.500 & 1.700 & 0.083 & 0.015 & 0.981 & 12.048 \\
\hline$X 128 \_36$ & 7.040 & 0.530 & 0.083 & 0.004 & 0.099 & 12.019 \\
\hline$X 128 \_37$ & 11.500 & 1.100 & 0.119 & 0.011 & 0.761 & 8.403 \\
\hline X128_38 & 52.700 & 5.000 & 0.456 & 0.050 & 0.360 & 2.193 \\
\hline X128_39 & 6.090 & 0.390 & 0.062 & 0.003 & 0.476 & 16.026 \\
\hline$X 128 \_40$ & 336.000 & 43.000 & 2.840 & 0.370 & 0.993 & 0.352 \\
\hline$X 128 \_41$ & 52.500 & 5.700 & 0.455 & 0.044 & 0.965 & 2.198 \\
\hline$X 128 \_42$ & 76.100 & 6.800 & 0.667 & 0.062 & 0.948 & 1.499 \\
\hline$X 128 \_43$ & 48.600 & 4.800 & 0.428 & 0.042 & -0.013 & 2.336 \\
\hline$X 128 \_44$ & 7.250 & 0.610 & 0.077 & 0.008 & 0.571 & 12.920 \\
\hline$X 128 \_45$ & 8.320 & 0.500 & 0.080 & 0.004 & 0.139 & 12.484 \\
\hline$X 128 \_46$ & 8.600 & 0.590 & 0.081 & 0.005 & 0.591 & 12.285 \\
\hline$X 128 \_47$ & 95.300 & 5.800 & 0.841 & 0.052 & 0.956 & 1.189 \\
\hline$X 128 \_48$ & 21.580 & 0.790 & 0.220 & 0.009 & 0.115 & 4.554 \\
\hline$X 128 \_49$ & 12.100 & 0.860 & 0.137 & 0.008 & 0.502 & 7.321 \\
\hline$X 128 \_50$ & 78.500 & 5.400 & 0.703 & 0.046 & 0.931 & 1.422 \\
\hline$X 128 \_51$ & 19.800 & 1.100 & 0.205 & 0.025 & 0.004 & 4.878 \\
\hline X128_52 & 11.100 & 1.500 & 0.135 & 0.015 & 0.968 & 7.407 \\
\hline$X 128 \_53$ & 9.350 & 0.580 & 0.108 & 0.005 & -0.435 & 9.268 \\
\hline$X 128 \_54$ & 9.250 & 0.330 & 0.116 & 0.004 & 0.841 & 8.621 \\
\hline$X 128 \_55$ & 4.890 & 0.690 & 0.051 & 0.006 & 0.024 & 19.646 \\
\hline X128_56 & 10.450 & 0.570 & 0.109 & 0.005 & 0.387 & 9.158 \\
\hline$X 128 \_57$ & 9.220 & 0.440 & 0.118 & 0.005 & 0.689 & 8.503 \\
\hline$X 128 \_58$ & 59.800 & 4.700 & 0.534 & 0.049 & 0.178 & 1.873 \\
\hline X128_59 & 131.000 & 12.000 & 1.102 & 0.093 & 0.140 & 0.907 \\
\hline$X 128 \_60$ & 25.100 & 3.300 & 0.246 & 0.027 & 0.769 & 4.065 \\
\hline$X 128 \_61$ & 7.140 & 0.500 & 0.068 & 0.005 & 0.383 & 14.706 \\
\hline$X 128 \_62$ & 8.740 & 0.540 & 0.092 & 0.005 & 0.839 & 10.881 \\
\hline X128_63 & 4.600 & 0.350 & 0.044 & 0.005 & -0.025 & 22.831 \\
\hline$X 128 \_64$ & 16.800 & 1.300 & 0.158 & 0.013 & 0.879 & 6.329 \\
\hline$X 128 \_65$ & 3.300 & 2.500 & 0.022 & 0.002 & 0.292 & 44.643 \\
\hline$X 128 \_66$ & 3.350 & 0.350 & 0.036 & 0.003 & -0.036 & 28.011 \\
\hline X128_67 & 7.290 & 0.410 & 0.073 & 0.004 & 0.277 & 13.793 \\
\hline X128_68 & 3.750 & 0.490 & 0.051 & 0.025 & 0.034 & 19.608 \\
\hline$X 128 \_69$ & 24.500 & 1.600 & 0.218 & 0.012 & 0.801 & 4.587 \\
\hline X128_70 & 362.000 & 16.000 & 3.190 & 0.160 & 0.305 & 0.313 \\
\hline X128_71 & 4.550 & 0.440 & 0.072 & 0.005 & 0.907 & 13.870 \\
\hline
\end{tabular}




$\begin{array}{lcccccc}\text { X128_72 } & 10.790 & 0.860 & 0.122 & 0.007 & 0.807 & 8.183 \\ \text { X128_73 } & 9.270 & 0.640 & 0.116 & 0.006 & 0.867 & 8.621 \\ \text { X128_74 } & 9.550 & 0.490 & 0.122 & 0.006 & 0.353 & 8.197 \\ \text { X128_75 } & 9.000 & 0.790 & 0.101 & 0.008 & 0.975 & 9.940 \\ \text { X128_76 } & 10.230 & 0.900 & 0.115 & 0.006 & 0.609 & 8.688 \\ \text { X128_77 } & 6.220 & 0.500 & 0.071 & 0.005 & 0.385 & 14.104 \\ \text { X128_78 } & 4.000 & 0.290 & 0.055 & 0.003 & 0.161 & 18.282 \\ \text { X128_79 } & 25.400 & 1.400 & 0.239 & 0.013 & 0.333 & 4.184 \\ \text { X128_80 } & 15.000 & 1.000 & 0.145 & 0.009 & 0.604 & 6.878\end{array}$


Page 108 of 203 


\begin{tabular}{|c|c|c|c|c|c|c|}
\hline Error $2 \sigma$ & ${ }^{207} \mathrm{~Pb} /{ }^{206} \mathrm{~Pb}$ & Error $2 \sigma$ & Jrrelation_38_ & lalAge ${ }^{206} \mathrm{~Pb} /{ }^{23}$ & Error $2 \sigma$ & FinalAge ${ }^{207} \mathrm{Pl}$ \\
\hline 0.679 & 0.058 & 0.010 & 0.037 & 540.735 & 32.135 & 526.774 \\
\hline 0.507 & 0.079 & 0.015 & 0.081 & 580.329 & 27.723 & 688.464 \\
\hline 0.674 & 0.062 & 0.011 & 0.154 & 537.770 & 31.524 & 574.010 \\
\hline 0.466 & 0.077 & 0.008 & 0.072 & 489.554 & 17.994 & 640.983 \\
\hline 0.746 & 0.106 & 0.092 & 0.095 & 521.738 & 32.802 & 845.722 \\
\hline 0.616 & 0.058 & 0.010 & 0.210 & 493.735 & 24.191 & 526.774 \\
\hline 0.760 & 0.090 & 0.052 & 0.039 & 535.398 & 35.240 & 728.882 \\
\hline 1.718 & 0.130 & 0.110 & 0.002 & 537.770 & 80.356 & 800.586 \\
\hline 0.509 & 0.052 & 0.010 & -0.424 & 534.211 & 23.495 & 477.234 \\
\hline 0.701 & 0.064 & 0.009 & -0.210 & 475.198 & 25.468 & 521.928 \\
\hline 1.559 & 0.180 & 0.150 & -0.168 & 519.954 & 68.089 & 2098.657 \\
\hline 0.579 & 0.062 & 0.009 & 0.217 & 469.207 & 20.508 & 507.863 \\
\hline 0.534 & 0.089 & 0.041 & 0.095 & 487.761 & 20.479 & 814.339 \\
\hline 0.612 & 0.083 & 0.035 & -0.362 & 524.710 & 27.226 & 672.882 \\
\hline 0.301 & 0.052 & 0.007 & -0.032 & 516.980 & 13.002 & 486.080 \\
\hline 1.219 & 0.074 & 0.026 & -0.024 & 519.954 & 53.233 & 850.127 \\
\hline 0.194 & 0.822 & 0.023 & 0.002 & 3965.741 & 653.181 & 4686.115 \\
\hline 0.046 & 0.828 & 0.015 & 0.333 & 5855.011 & 395.609 & 5208.812 \\
\hline 0.041 & 0.822 & 0.021 & 0.306 & 4752.065 & 213.625 & 4882.074 \\
\hline 0.047 & 0.836 & 0.017 & 0.096 & 4383.961 & 202.544 & 4800.109 \\
\hline 0.026 & 0.828 & 0.018 & 0.412 & 6965.017 & 350.756 & 5503.470 \\
\hline 0.037 & 0.834 & 0.043 & 0.366 & 7735.470 & 666.851 & 5622.515 \\
\hline 3.431 & 0.661 & 0.063 & -0.390 & 255.312 & 35.390 & 1575.680 \\
\hline 0.088 & 0.828 & 0.022 & 0.168 & 2928.318 & 147.689 & 4246.383 \\
\hline 0.081 & 0.821 & 0.025 & -0.017 & 6774.031 & 1019.747 & 5404.894 \\
\hline 0.033 & 0.835 & 0.021 & 0.176 & 4334.789 & 135.603 & 4790.177 \\
\hline 0.017 & 0.842 & 0.017 & 0.328 & 8112.561 & 354.120 & 5764.080 \\
\hline 0.039 & 0.820 & 0.017 & 0.063 & 4599.129 & 185.556 & 4846.651 \\
\hline 0.027 & 0.819 & 0.012 & 0.255 & 7518.266 & 442.251 & 5606.398 \\
\hline 0.033 & 0.825 & 0.014 & -0.199 & 5070.991 & 199.278 & 4999.364 \\
\hline 0.011 & 0.832 & 0.011 & 0.320 & 8834.289 & 288.761 & 5909.614 \\
\hline 0.019 & 0.821 & 0.015 & 0.120 & 8257.430 & 412.872 & 5794.903 \\
\hline 0.049 & 0.832 & 0.015 & 0.103 & 6059.676 & 466.129 & 5255.775 \\
\hline 0.042 & 0.835 & 0.019 & 0.393 & 5691.802 & 337.173 & 5202.786 \\
\hline 0.030 & 0.817 & 0.021 & 0.120 & 6277.548 & 308.545 & 5346.274 \\
\hline 0.028 & 0.816 & 0.024 & 0.045 & 6701.497 & 348.273 & 5429.362 \\
\hline 0.026 & 0.826 & 0.014 & 0.105 & 6868.011 & 339.428 & 5476.598 \\
\hline 0.019 & 0.839 & 0.022 & 0.503 & 7869.975 & 362.216 & 5692.036 \\
\hline 0.021 & 0.821 & 0.012 & 0.072 & 10652.683 & 934.003 & 6266.640 \\
\hline 0.039 & 0.830 & 0.016 & 0.028 & 7617.903 & 674.151 & 5692.036 \\
\hline 0.048 & 0.841 & 0.020 & 0.245 & 7376.134 & 758.294 & 5585.884 \\
\hline 0.041 & 0.820 & 0.022 & 0.535 & 6450.488 & 450.034 & 5333.069 \\
\hline 0.042 & 0.815 & 0.027 & -0.292 & 6209.021 & 421.600 & 5322.381 \\
\hline 0.027 & 0.828 & 0.019 & 0.432 & 5867.995 & 237.091 & 5223.130 \\
\hline 0.028 & 0.830 & 0.025 & -0.040 & 5608.621 & 214.316 & 5124.931 \\
\hline 0.142 & 0.818 & 0.030 & 0.329 & 3137.714 & 280.242 & 4379.835 \\
\hline 0.030 & 0.838 & 0.023 & 0.295 & 7538.317 & 509.346 & 5610.451 \\
\hline 0.205 & 0.820 & 0.025 & 0.700 & 6660.335 & 2465.428 & 5127.538 \\
\hline
\end{tabular}




\begin{tabular}{|c|c|c|c|c|c|c|}
\hline 0.025 & 0.833 & 0.020 & 0.054 & 8094.221 & 515.966 & 5757.101 \\
\hline 0.035 & 0.829 & 0.025 & 0.310 & 6087.316 & 337.108 & 5278.466 \\
\hline 0.058 & 0.844 & 0.020 & 0.201 & 6034.446 & 545.047 & 5295.157 \\
\hline 0.014 & 0.843 & 0.016 & 0.234 & 9312.253 & 431.123 & 6018.100 \\
\hline 0.020 & 0.831 & 0.022 & 0.196 & 8185.402 & 415.665 & 5781.319 \\
\hline 0.023 & 0.817 & 0.025 & 0.573 & 6769.522 & 287.832 & 5422.084 \\
\hline 0.108 & 0.803 & 0.025 & 0.266 & 3424.440 & 258.909 & 4413.260 \\
\hline 0.950 & 0.591 & 0.057 & 0.237 & 713.273 & 79.253 & 2387.547 \\
\hline 0.460 & 0.775 & 0.047 & -0.285 & 2001.106 & 335.350 & 3704.176 \\
\hline 0.422 & 0.764 & 0.047 & 0.235 & 1297.707 & 122.206 & 3252.014 \\
\hline 0.418 & 0.660 & 0.032 & -0.286 & 854.268 & 50.641 & 2708.258 \\
\hline 0.039 & 0.803 & 0.023 & 0.249 & 4059.144 & 138.853 & 4642.988 \\
\hline 0.101 & 0.824 & 0.018 & 0.074 & 4010.882 & 348.570 & 4676.012 \\
\hline 0.065 & 0.805 & 0.017 & 0.104 & 5958.156 & 587.976 & 5202.786 \\
\hline 0.048 & 0.835 & 0.015 & 0.136 & 4117.267 & 175.007 & 4728.449 \\
\hline 0.060 & 0.836 & 0.031 & 0.526 & 4218.574 & 232.843 & 4720.706 \\
\hline 0.017 & 0.842 & 0.025 & 0.061 & 10258.011 & 682.118 & 6218.900 \\
\hline 0.016 & 0.832 & 0.018 & 0.454 & 8984.795 & 444.792 & 5962.449 \\
\hline 0.260 & 0.805 & 0.022 & 0.215 & 1799.489 & 150.889 & 3649.864 \\
\hline 0.035 & 0.848 & 0.009 & 0.217 & 7735.470 & 633.508 & 5695.763 \\
\hline 0.302 & 0.778 & 0.025 & -0.078 & 1910.679 & 199.375 & 3696.212 \\
\hline 0.487 & 0.787 & 0.029 & -0.142 & 1292.434 & 139.723 & 3264.322 \\
\hline 0.149 & 0.798 & 0.038 & 0.305 & 2452.790 & 169.523 & 4021.743 \\
\hline 0.072 & 0.817 & 0.027 & 0.084 & 3518.495 & 184.164 & 4490.479 \\
\hline 0.120 & 0.800 & 0.025 & 0.425 & 3336.681 & 270.675 & 4368.902 \\
\hline 0.042 & 0.811 & 0.024 & 0.308 & 4831.765 & 225.136 & 4917.105 \\
\hline 0.105 & 0.802 & 0.024 & 0.277 & 4041.949 & 370.821 & 4668.879 \\
\hline 0.021 & 0.839 & 0.009 & 0.267 & 6132.291 & 200.679 & 5295.157 \\
\hline 0.023 & 0.844 & 0.008 & -0.273 & 8203.485 & 478.803 & 5804.973 \\
\hline 0.071 & 0.836 & 0.019 & 0.189 & 4718.047 & 362.927 & 4900.962 \\
\hline 0.032 & 0.838 & 0.015 & -0.071 & 6410.071 & 346.287 & 5348.894 \\
\hline 0.047 & 0.829 & 0.019 & 0.295 & 5132.344 & 295.205 & 5024.887 \\
\hline 0.034 & 0.809 & 0.017 & 0.312 & 5000.151 & 200.518 & 4972.425 \\
\hline 0.048 & 0.832 & 0.024 & 0.333 & 5014.974 & 281.213 & 4990.464 \\
\hline 0.035 & 0.841 & 0.021 & 0.332 & 6366.999 & 370.306 & 5343.646 \\
\hline 0.057 & 0.828 & 0.023 & 0.329 & 4822.619 & 303.309 & 4952.515 \\
\hline 0.061 & 0.817 & 0.022 & 0.327 & 3424.440 & 146.552 & 4483.139 \\
\hline 0.046 & 0.850 & 0.018 & 0.333 & 5161.356 & 290.247 & 5042.025 \\
\hline 0.059 & 0.808 & 0.022 & 0.248 & 4807.347 & 312.391 & 4906.641 \\
\hline 0.037 & 0.837 & 0.018 & -0.098 & 5447.281 & 266.621 & 5112.456 \\
\hline 0.020 & 0.831 & 0.019 & 0.315 & 7163.252 & 284.702 & 5553.032 \\
\hline 0.410 & 0.823 & 0.020 & 0.085 & 2269.552 & 392.600 & 3907.212 \\
\hline 0.035 & 0.839 & 0.029 & 0.610 & 5158.461 & 218.793 & 5050.486 \\
\hline 0.033 & 0.835 & 0.018 & 0.423 & 5355.248 & 227.443 & 5081.604 \\
\hline 0.040 & 0.837 & 0.020 & 0.293 & 6497.714 & 448.118 & 5379.822 \\
\hline 0.025 & 0.847 & 0.016 & -0.081 & 10165.445 & 979.483 & 6229.949 \\
\hline 0.015 & 0.856 & 0.008 & 0.004 & 12290.573 & 1029.577 & 6617.933 \\
\hline 0.109 & 0.834 & 0.028 & 0.195 & 4752.065 & 566.760 & 4844.072 \\
\hline 0.082 & 0.846 & 0.019 & 0.281 & 5111.958 & 506.971 & 5046.264 \\
\hline
\end{tabular}




\begin{tabular}{|c|c|c|c|c|c|c|}
\hline 0.054 & 0.838 & 0.024 & 0.580 & 5880.952 & 473.634 & 5167.738 \\
\hline 0.148 & 0.868 & 0.026 & 0.057 & 5643.634 & 1169.038 & 5278.466 \\
\hline 0.018 & 0.847 & 0.016 & 0.361 & 8554.875 & 432.376 & 5875.918 \\
\hline 0.048 & 0.849 & 0.012 & -0.068 & 5776.555 & 398.383 & 5220.758 \\
\hline 0.029 & 0.850 & 0.013 & 0.455 & 5860.207 & 249.118 & 5248.290 \\
\hline 0.161 & 0.841 & 0.017 & -0.154 & 5425.091 & 1150.777 & 5107.821 \\
\hline 0.022 & 0.842 & 0.014 & -0.271 & 5899.049 & 193.088 & 5224.315 \\
\hline 0.078 & 0.852 & 0.018 & 0.226 & 4745.893 & 401.307 & 4956.375 \\
\hline 0.051 & 0.848 & 0.008 & 0.150 & 6009.116 & 468.243 & 5278.466 \\
\hline 0.012 & 0.843 & 0.009 & -0.062 & 12451.387 & 886.370 & 6631.342 \\
\hline 0.028 & 0.851 & 0.015 & -0.142 & 9976.229 & 1024.586 & 6200.968 \\
\hline 0.059 & 0.845 & 0.027 & 0.709 & 5000.151 & 345.574 & 4981.485 \\
\hline 0.025 & 0.847 & 0.016 & -0.160 & 8639.809 & 612.752 & 5912.622 \\
\hline 0.118 & 0.823 & 0.016 & -0.029 & 4627.493 & 572.928 & 4861.138 \\
\hline 0.060 & 0.827 & 0.027 & 0.688 & 3492.298 & 150.572 & 4478.216 \\
\hline 0.047 & 0.841 & 0.023 & 0.419 & 4913.500 & 266.524 & 4978.474 \\
\hline 0.027 & 0.824 & 0.020 & 0.097 & 8622.912 & 644.417 & 5844.283 \\
\hline 0.130 & 0.830 & 0.021 & -0.583 & 4205.158 & 502.791 & 4735.177 \\
\hline 0.032 & 0.836 & 0.021 & 0.407 & 6026.857 & 299.979 & 5289.624 \\
\hline 0.052 & 0.814 & 0.021 & 0.422 & 4248.659 & 204.919 & 4728.449 \\
\hline 0.115 & 0.818 & 0.024 & 0.386 & 3522.229 & 295.538 & 4481.911 \\
\hline 0.021 & 0.844 & 0.017 & -0.013 & 9111.510 & 585.949 & 5990.656 \\
\hline 0.007 & 0.837 & 0.013 & 0.475 & 14666.971 & 940.837 & 7008.928 \\
\hline 0.008 & 0.871 & 0.014 & 0.526 & 13688.693 & 855.543 & 6885.371 \\
\hline 0.014 & 0.848 & 0.015 & 0.387 & 9921.131 & 515.031 & 6128.326 \\
\hline 0.017 & 0.848 & 0.016 & 0.000 & 10452.000 & 720.828 & 6279.280 \\
\hline 0.035 & 0.835 & 0.018 & 0.079 & 6751.452 & 437.932 & 5457.967 \\
\hline 0.028 & 0.845 & 0.023 & 0.297 & 6818.954 & 362.710 & 5457.967 \\
\hline 0.339 & 0.834 & 0.025 & -0.082 & 2907.820 & 561.158 & 4254.104 \\
\hline 0.824 & 0.814 & 0.019 & 0.090 & 1505.172 & 326.216 & 3476.938 \\
\hline 0.215 & 0.804 & 0.023 & 0.159 & 5023.851 & 1277.250 & 4957.914 \\
\hline 0.093 & 0.812 & 0.022 & 0.181 & 3709.841 & 267.032 & 4566.772 \\
\hline 0.038 & 0.795 & 0.049 & 0.145 & 10003.602 & 1425.244 & 6098.749 \\
\hline 0.966 & 0.574 & 0.030 & -0.432 & 603.265 & 57.190 & 2178.942 \\
\hline 1.491 & 0.698 & 0.065 & 0.331 & 362.842 & 31.333 & 1916.099 \\
\hline 0.476 & 0.790 & 0.060 & 0.563 & 912.165 & 66.012 & 2906.230 \\
\hline 2.613 & 0.806 & 0.038 & 0.086 & 514.005 & 111.471 & 2377.830 \\
\hline 1.957 & 0.443 & 0.035 & -0.499 & 251.593 & 19.596 & 1294.982 \\
\hline 1.099 & 0.578 & 0.034 & 0.420 & 511.623 & 46.455 & 2044.544 \\
\hline 2.479 & 0.759 & 0.068 & -0.062 & 1281.875 & 699.205 & 2940.460 \\
\hline 0.649 & 0.687 & 0.034 & -0.049 & 940.083 & 95.805 & 2808.874 \\
\hline 1.233 & 0.777 & 0.038 & 0.428 & 889.742 & 162.318 & 2796.020 \\
\hline 0.406 & 0.793 & 0.036 & -0.013 & 3789.116 & 1231.463 & 4725.552 \\
\hline 1.272 & 0.740 & 0.048 & 0.340 & 573.255 & 67.804 & 2368.019 \\
\hline 1.245 & 0.855 & 0.084 & 0.801 & 579.150 & 67.773 & 2523.132 \\
\hline 0.300 & 0.820 & 0.090 & 0.576 & 2314.727 & 300.057 & 3917.980 \\
\hline 0.735 & 0.630 & 0.024 & 0.159 & 418.056 & 20.591 & 1935.904 \\
\hline 0.720 & 0.632 & 0.054 & 0.534 & 268.311 & 8.207 & 1586.380 \\
\hline 1.214 & 0.814 & 0.087 & 0.556 & 468.607 & 42.883 & 2266.500 \\
\hline
\end{tabular}




\begin{tabular}{|c|c|c|c|c|c|c|}
\hline 0.657 & 0.687 & 0.055 & 0.175 & 650.058 & 45.339 & 2453.984 \\
\hline 0.414 & 0.730 & 0.021 & 0.095 & 566.764 & 21.585 & 2376.853 \\
\hline 0.440 & 0.743 & 0.031 & 0.193 & 755.266 & 41.318 & 2647.230 \\
\hline 0.383 & 0.738 & 0.023 & -0.151 & 904.886 & 52.240 & 2827.853 \\
\hline 0.262 & 0.802 & 0.023 & -0.077 & 1982.174 & 187.205 & 3743.084 \\
\hline 0.164 & 0.727 & 0.025 & 0.173 & 1017.068 & 28.566 & 2947.170 \\
\hline 0.302 & 0.686 & 0.028 & 0.066 & 901.524 & 40.842 & 2761.151 \\
\hline 1.508 & 0.694 & 0.058 & 0.386 & 335.362 & 27.005 & 1855.887 \\
\hline 0.233 & 0.841 & 0.018 & -0.056 & 4305.105 & 951.655 & 4744.711 \\
\hline 2.261 & 0.653 & 0.053 & -0.159 & 252.213 & 22.756 & 1564.867 \\
\hline 0.601 & 0.720 & 0.038 & 0.488 & 583.863 & 33.258 & 2384.641 \\
\hline 0.427 & 0.775 & 0.049 & -0.042 & 1234.143 & 111.131 & 3201.235 \\
\hline 1.200 & 0.790 & 0.031 & 0.076 & 724.805 & 103.544 & 2679.654 \\
\hline 2.177 & 0.654 & 0.038 & -0.490 & 514.005 & 92.892 & 2172.987 \\
\hline 0.592 & 0.608 & 0.053 & 0.555 & 515.195 & 25.388 & 2116.494 \\
\hline 0.777 & 0.687 & 0.040 & 0.212 & 724.805 & 66.999 & 2564.582 \\
\hline 0.240 & 0.870 & 0.035 & 0.387 & 2421.872 & 265.556 & 4044.690 \\
\hline 0.770 & 0.697 & 0.039 & 0.239 & 390.205 & 18.760 & 1988.816 \\
\hline 0.046 & 0.859 & 0.014 & 0.040 & 8673.472 & 1129.995 & 5909.614 \\
\hline 0.213 & 0.815 & 0.019 & -0.133 & 2417.443 & 233.775 & 4040.901 \\
\hline 0.139 & 0.825 & 0.026 & 0.064 & 3294.283 & 306.215 & 4411.944 \\
\hline 0.229 & 0.831 & 0.022 & 0.371 & 2296.695 & 225.377 & 3964.046 \\
\hline 1.319 & 0.671 & 0.072 & 0.449 & 480.585 & 49.052 & 2142.675 \\
\hline 0.655 & 0.756 & 0.046 & 0.577 & 496.720 & 26.045 & 2266.500 \\
\hline 0.800 & 0.751 & 0.039 & 0.418 & 504.474 & 32.847 & 2296.556 \\
\hline 0.074 & 0.830 & 0.014 & 0.200 & 3934.304 & 243.263 & 4637.730 \\
\hline 0.191 & 0.700 & 0.021 & 0.381 & 1279.761 & 53.615 & 3165.015 \\
\hline 0.402 & 0.655 & 0.045 & 0.342 & 825.408 & 45.319 & 2612.187 \\
\hline 0.093 & 0.808 & 0.018 & 0.177 & 3432.015 & 224.570 & 4443.070 \\
\hline 0.595 & 0.717 & 0.047 & 0.018 & 1202.125 & 146.601 & 3081.640 \\
\hline 0.823 & 0.604 & 0.020 & -0.357 & 816.327 & 90.703 & 2531.559 \\
\hline 0.438 & 0.640 & 0.040 & 0.879 & 660.540 & 31.221 & 2372.937 \\
\hline 0.312 & 0.588 & 0.010 & 0.745 & 707.500 & 25.616 & 2363.078 \\
\hline 2.432 & 0.654 & 0.030 & 0.329 & 320.045 & 39.613 & 1800.534 \\
\hline 0.453 & 0.708 & 0.032 & 0.143 & 668.100 & 33.038 & 2475.493 \\
\hline 0.347 & 0.570 & 0.019 & -0.307 & 716.735 & 29.254 & 2360.102 \\
\hline 0.172 & 0.819 & 0.027 & 0.069 & 2758.283 & 253.101 & 4170.777 \\
\hline 0.077 & 0.845 & 0.021 & -0.084 & 4788.972 & 404.151 & 4957.914 \\
\hline 0.446 & 0.757 & 0.067 & 0.513 & 1417.814 & 155.614 & 3312.114 \\
\hline 1.146 & 0.768 & 0.071 & 0.683 & 424.095 & 33.054 & 2129.045 \\
\hline 0.533 & 0.691 & 0.031 & 0.225 & 566.764 & 27.752 & 2311.257 \\
\hline 2.658 & 0.760 & 0.080 & -0.041 & 276.344 & 32.177 & 1749.268 \\
\hline 0.521 & 0.765 & 0.039 & 0.426 & 945.653 & 77.807 & 2923.489 \\
\hline 3.388 & 0.682 & 0.095 & -0.156 & 142.806 & 10.838 & 1481.053 \\
\hline 2.354 & 0.700 & 0.110 & 0.753 & 226.124 & 19.002 & 1492.792 \\
\hline 0.685 & 0.726 & 0.043 & 0.444 & 451.200 & 22.404 & 2147.586 \\
\hline 9.612 & 0.761 & 0.059 & 0.121 & 320.658 & 157.185 & 1582.114 \\
\hline 0.253 & 0.820 & 0.030 & -0.110 & 1271.298 & 69.980 & 3288.499 \\
\hline 0.016 & 0.843 & 0.015 & 0.607 & 9235.782 & 463.237 & 5985.077 \\
\hline $0 . \varsigma$ & 56 & 0.018 & -0.237 & 448.795 & 30.501 & 1740.161 \\
\hline
\end{tabular}




\begin{tabular}{llll|lll}
0.469 & 0.630 & 0.029 & 0.105 & 743.214 & 42.574 & 2505.206 \\
0.461 & 0.592 & 0.018 & -0.188 & 707.500 & 37.815 & 2365.058 \\
0.383 & 0.571 & 0.019 & 0.372 & 742.065 & 34.670 & 2392.370 \\
0.790 & 0.644 & 0.013 & -0.370 & 617.924 & 49.139 & 2338.006 \\
0.438 & 0.654 & 0.051 & 0.300 & 702.299 & 35.390 & 2455.794 \\
0.935 & 0.648 & 0.065 & 0.406 & 441.576 & 29.272 & 2007.265 \\
0.836 & 0.553 & 0.050 & 0.313 & 343.313 & 15.691 & 1634.196 \\
0.228 & 0.765 & 0.032 & 0.541 & 1381.496 & 75.144 & 3323.718 \\
0.416 & 0.746 & 0.046 & 0.302 & 875.126 & 52.965 & 2815.240
\end{tabular}


Page 114 of 203 
Page 115 of 203

Page 115 of 203
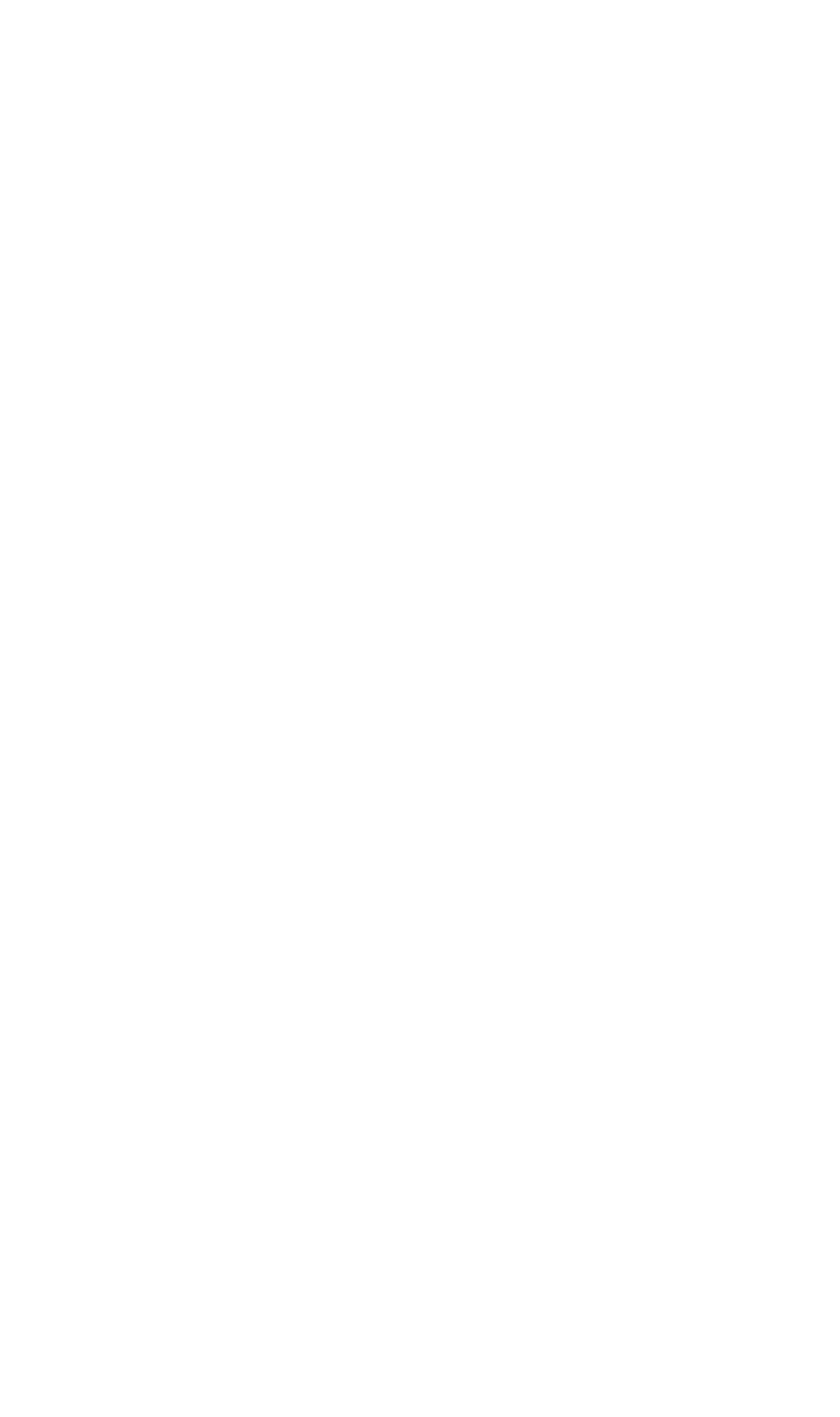


\begin{tabular}{|c|c|c|c|c|}
\hline Error $2 \sigma$ & FinalAge ${ }^{207} \mathrm{~Pb}$ & Error $2 \sigma$ & FinalAge ${ }^{207} \mathrm{~Pb}$ corr & Error $2 \sigma$ \\
\hline 92.960 & 529.762 & 91.338 & 540.924 & 32.029 \\
\hline 106.463 & 1172.135 & 222.557 & 566.699 & 28.373 \\
\hline 90.633 & 674.109 & 119.600 & 535.329 & 31.437 \\
\hline 72.839 & 1131.515 & 122.800 & 477.481 & 17.868 \\
\hline 650.555 & 1731.725 & 1503.007 & 491.426 & 65.318 \\
\hline 100.707 & 518.389 & 88.944 & 493.353 & 24.349 \\
\hline 402.621 & 1425.530 & 823.639 & 514.901 & 47.089 \\
\hline 733.871 & 2097.963 & 1775.199 & 491.219 & 101.274 \\
\hline 87.493 & 294.181 & 55.793 & 537.999 & 23.945 \\
\hline 73.008 & 744.932 & 101.106 & 470.887 & 25.189 \\
\hline 1916.165 & 2652.870 & 2210.725 & 442.861 & 110.598 \\
\hline 69.645 & 687.846 & 98.106 & 465.824 & 20.551 \\
\hline 450.204 & 1404.160 & 646.860 & 468.862 & 30.898 \\
\hline 257.700 & 1269.216 & 535.211 & 508.853 & 33.964 \\
\hline 67.291 & 298.548 & 39.388 & 520.308 & 13.476 \\
\hline 616.504 & 1041.468 & 365.921 & 509.792 & 53.515 \\
\hline 796.640 & 4960.341 & 138.793 & 173.354 & 166.386 \\
\hline 310.048 & 4970.679 & 90.049 & 448.415 & 192.587 \\
\hline 233.054 & 4960.341 & 126.724 & 290.458 & 190.335 \\
\hline 227.148 & 4984.340 & 101.356 & 16.214 & 149.925 \\
\hline 237.366 & 4970.679 & 108.058 & 1213.151 & 266.439 \\
\hline 488.914 & 4980.938 & 256.811 & 1787.042 & 681.328 \\
\hline 296.499 & 4648.179 & 443.019 & 58.375 & 22.183 \\
\hline 250.174 & 4970.679 & 132.071 & 52.099 & 109.274 \\
\hline 794.837 & 4958.610 & 150.993 & 1428.435 & 383.614 \\
\hline 172.775 & 4982.640 & 125.312 & 29.716 & 175.671 \\
\hline 257.502 & 4994.495 & 100.839 & 1349.044 & 325.782 \\
\hline 214.856 & 4956.877 & 102.765 & 294.640 & 152.131 \\
\hline 315.219 & 4955.142 & 72.603 & 3025.584 & 362.410 \\
\hline 201.440 & 4965.520 & 84.263 & 298.413 & 150.266 \\
\hline 193.469 & 4977.527 & 65.809 & \#VALUE! & \#VALUE! \\
\hline 328.378 & 4958.610 & 90.596 & 305.523 & 352.334 \\
\hline 418.073 & 4977.527 & 89.739 & 377.927 & 205.139 \\
\hline 277.274 & 4982.640 & 113.377 & 163.983 & 232.276 \\
\hline 263.842 & 4951.665 & 127.277 & 1120.597 & 257.702 \\
\hline 311.734 & 4949.923 & 145.586 & 1614.651 & 311.359 \\
\hline 300.088 & 4967.242 & 84.191 & 1236.386 & 215.693 \\
\hline 231.042 & 4989.427 & 130.831 & 1385.310 & 380.925 \\
\hline 550.625 & 4958.610 & 72.477 & 0.000 & 575.783 \\
\hline 231.042 & 4974.107 & 95.886 & 2019.055 & 319.781 \\
\hline 549.431 & 4992.807 & 118.735 & 555.995 & 346.578 \\
\hline 308.757 & 4956.877 & 132.989 & 1142.588 & 284.318 \\
\hline 452.969 & 4948.178 & 163.927 & 1142.035 & 318.379 \\
\hline 190.043 & 4970.679 & 114.061 & 453.505 & 231.514 \\
\hline 298.347 & 4974.107 & 149.823 & 302.436 & 281.536 \\
\hline 415.995 & 4953.404 & 181.665 & 133.295 & 155.659 \\
\hline 381.511 & 4987.733 & 136.895 & 1034.133 & 381.328 \\
\hline 396.971 & 4956.877 & 151.124 & 1353.427 & 577.371 \\
\hline
\end{tabular}




\begin{tabular}{|c|c|c|c|c|}
\hline 378.495 & 4979.233 & 119.549 & 2833.533 & 480.231 \\
\hline 293.248 & 4972.394 & 149.952 & 509.851 & 308.671 \\
\hline 462.965 & 4997.862 & 118.433 & -130.682 & 279.598 \\
\hline 257.459 & 4996.180 & 94.827 & -197.347 & 489.098 \\
\hline 292.972 & 4975.818 & 131.730 & 0.000 & 440.307 \\
\hline 229.949 & 4951.665 & 151.520 & 1649.011 & 323.759 \\
\hline 306.959 & 4927.067 & 153.396 & 284.903 & 144.066 \\
\hline 326.717 & 4486.026 & 432.662 & 240.881 & 58.641 \\
\hline 752.720 & 4876.472 & 295.734 & 215.986 & 140.621 \\
\hline 399.612 & 4856.061 & 298.737 & 144.690 & 85.455 \\
\hline 222.320 & 4645.995 & 225.260 & 213.151 & 38.401 \\
\hline 159.936 & 4927.067 & 141.124 & 410.642 & 162.251 \\
\hline 425.092 & 4963.796 & 108.432 & 154.381 & 136.028 \\
\hline 498.471 & 4930.609 & 104.125 & 1277.305 & 225.483 \\
\hline 208.541 & 4982.640 & 89.509 & 22.592 & 124.609 \\
\hline 214.370 & 4984.340 & 184.826 & 11.632 & 241.326 \\
\hline 436.414 & 4994.495 & 148.293 & \#VALUE! & \#VALUE! \\
\hline 252.646 & 4977.527 & 107.687 & 89.122 & 485.991 \\
\hline 299.000 & 4930.609 & 134.750 & 95.542 & 61.001 \\
\hline 460.687 & 5004.405 & 53.709 & 6.502 & 231.721 \\
\hline 388.551 & 4881.985 & 156.876 & 192.831 & 73.859 \\
\hline 396.089 & 4898.390 & 180.500 & 98.343 & 54.494 \\
\hline 288.941 & 4918.171 & 234.199 & 183.128 & 142.392 \\
\hline 250.987 & 4951.665 & 163.641 & 176.617 & 161.963 \\
\hline 275.678 & 4921.737 & 153.804 & 294.494 & 139.534 \\
\hline 238.429 & 4941.178 & 146.225 & 508.316 & 211.543 \\
\hline 432.216 & 4925.292 & 147.390 & 418.053 & 170.942 \\
\hline 176.505 & 4990.104 & 50.531 & 85.941 & 151.551 \\
\hline 364.008 & 4998.535 & 45.581 & 1089.098 & 210.791 \\
\hline 376.084 & 4984.340 & 113.280 & 29.707 & 181.428 \\
\hline 304.859 & 4987.733 & 89.279 & 231.221 & 227.895 \\
\hline 287.136 & 4972.394 & 113.963 & 221.634 & 197.581 \\
\hline 209.523 & 4937.664 & 103.758 & 604.962 & 163.090 \\
\hline 250.814 & 4977.527 & 143.583 & 136.361 & 237.469 \\
\hline 306.146 & 4992.807 & 124.672 & 60.937 & 305.385 \\
\hline 307.869 & 4970.679 & 138.074 & 191.508 & 214.264 \\
\hline 181.082 & 4951.665 & 133.337 & 166.951 & 130.314 \\
\hline 269.097 & 5007.915 & 106.050 & -292.111 & 210.425 \\
\hline 331.051 & 4935.904 & 134.393 & 553.192 & 194.834 \\
\hline 287.931 & 4986.038 & 107.227 & 63.931 & 211.814 \\
\hline 197.483 & 4975.818 & 113.767 & 1222.400 & 288.797 \\
\hline 638.433 & 4962.070 & 120.585 & 55.597 & 74.346 \\
\hline 186.404 & 4989.427 & 172.459 & -12.717 & 301.791 \\
\hline 199.009 & 4982.640 & 107.410 & 108.172 & 204.905 \\
\hline 378.480 & 4986.038 & 119.141 & 318.962 & 289.842 \\
\hline 635.157 & 5002.898 & 94.506 & -364.081 & 592.728 \\
\hline 548.231 & 5018.556 & 49.225 & -5689.769 & 1570.951 \\
\hline 538.230 & 4980.938 & 167.226 & 69.460 & 256.182 \\
\hline 529.328 & 5001.222 & 112.321 & -185.542 & 212.711 \\
\hline
\end{tabular}




\begin{tabular}{|c|c|c|c|c|}
\hline 259.508 & 4987.733 & 142.847 & 94.260 & 302.335 \\
\hline 1172.992 & 5037.628 & 150.897 & -1044.046 & 443.883 \\
\hline 271.196 & 5002.898 & 94.506 & 1184.548 & 347.330 \\
\hline 368.524 & 5006.245 & 70.760 & -330.343 & 185.316 \\
\hline 225.313 & 5007.915 & 76.592 & -379.474 & 200.948 \\
\hline 1041.727 & 4992.807 & 100.925 & -53.996 & 205.832 \\
\hline 284.796 & 4994.495 & 83.044 & -57.780 & 200.528 \\
\hline 356.192 & 5011.250 & 105.871 & -284.534 & 187.699 \\
\hline 410.547 & 5004.573 & 47.803 & -307.365 & 156.854 \\
\hline 454.997 & 4995.843 & 52.756 & -7802.717 & 2332.840 \\
\hline 692.072 & 5009.583 & 88.301 & \#NUM! & \#VALUE! \\
\hline 230.315 & 4999.543 & 159.749 & -155.763 & 277.938 \\
\hline 456.167 & 5002.898 & 94.506 & 1362.315 & 356.185 \\
\hline 612.748 & 4962.070 & 96.468 & 250.041 & 150.150 \\
\hline 170.756 & 4968.962 & 162.227 & 84.012 & 163.119 \\
\hline 234.588 & 4992.807 & 136.545 & -64.932 & 230.631 \\
\hline 445.279 & 4963.796 & 120.480 & 262.991 & 483.616 \\
\hline 631.357 & 4974.107 & 125.851 & 94.115 & 166.802 \\
\hline 235.417 & 4984.340 & 125.205 & 205.445 & 273.680 \\
\hline 208.541 & 4946.432 & 127.611 & 315.205 & 162.390 \\
\hline 368.000 & 4953.404 & 145.332 & 167.972 & 146.441 \\
\hline 411.446 & 4997.862 & 100.668 & 0.000 & 481.163 \\
\hline 416.023 & 4986.038 & 77.441 & -3155.743 & 1795.990 \\
\hline 422.511 & 5042.516 & 81.051 & -2481.884 & 1398.378 \\
\hline 308.621 & 5004.573 & 88.524 & -377.805 & 538.007 \\
\hline 467.054 & 5004.573 & 94.426 & \#VALUE! & \#VALUE! \\
\hline 380.788 & 4982.640 & 107.410 & 571.313 & 272.291 \\
\hline 279.245 & 4999.543 & 136.082 & -78.477 & 377.323 \\
\hline 916.269 & 4980.938 & 149.309 & 12.180 & 122.352 \\
\hline 772.653 & 4946.432 & 115.457 & 53.430 & 45.422 \\
\hline 1286.787 & 4928.839 & 140.999 & 711.352 & 271.171 \\
\hline 344.565 & 4942.932 & 133.922 & 247.811 & 143.251 \\
\hline 873.401 & 4912.805 & 302.802 & \#VALUE! & \#VALUE! \\
\hline 274.172 & 4443.524 & 232.240 & 214.528 & 30.925 \\
\hline 195.031 & 4726.607 & 440.157 & 66.482 & 31.049 \\
\hline 211.362 & 4903.814 & 372.442 & 60.980 & 74.376 \\
\hline 505.921 & 4932.376 & 232.544 & 20.920 & 26.607 \\
\hline 195.753 & 4061.872 & 320.915 & 128.055 & 14.942 \\
\hline 201.619 & 4453.645 & 261.979 & 177.261 & 27.569 \\
\hline 240.739 & 4846.679 & 434.222 & 152.282 & 145.128 \\
\hline 339.327 & 4703.764 & 232.792 & 201.899 & 47.403 \\
\hline 380.411 & 4880.150 & 238.669 & 75.969 & 48.216 \\
\hline 1635.768 & 4909.216 & 222.865 & 450.846 & 264.043 \\
\hline 280.088 & 4810.418 & 312.027 & 75.528 & 37.426 \\
\hline 275.251 & 5016.235 & 492.823 & -15.672 & 65.017 \\
\hline 455.972 & 4956.877 & 544.048 & 70.717 & 314.665 \\
\hline 114.870 & 4578.760 & 174.429 & 114.759 & 14.409 \\
\hline 117.821 & 4583.348 & 391.615 & 71.561 & 18.737 \\
\hline 234.278 & 4946.432 & 528.673 & 13.827 & 53.666 \\
\hline
\end{tabular}




\begin{tabular}{|c|c|c|c|c|}
\hline 221.123 & 4703.764 & 376.575 & 133.839 & 47.731 \\
\hline 111.375 & 4790.932 & 137.821 & 82.315 & 16.705 \\
\hline 151.752 & 4816.209 & 200.945 & 99.169 & 32.200 \\
\hline 204.647 & 4806.544 & 149.797 & 128.112 & 29.912 \\
\hline 346.404 & 4925.292 & 141.249 & 119.771 & 70.884 \\
\hline 116.381 & 4785.031 & 164.547 & 162.759 & 35.508 \\
\hline 163.682 & 4701.668 & 191.905 & 193.780 & 35.145 \\
\hline 156.435 & 4718.345 & 394.329 & 62.974 & 25.667 \\
\hline 1074.274 & 4992.807 & 106.862 & -60.601 & 156.173 \\
\hline 157.766 & 4630.604 & 375.838 & 60.263 & 18.031 \\
\hline 148.568 & 4771.159 & 251.811 & 92.966 & 29.878 \\
\hline 357.281 & 4876.472 & 308.319 & 115.518 & 84.267 \\
\hline 371.029 & 4903.814 & 192.428 & 46.954 & 31.521 \\
\hline 492.544 & 4632.813 & 269.185 & 126.464 & 34.150 \\
\hline 159.338 & 4527.234 & 394.644 & 158.215 & 36.017 \\
\hline 245.308 & 4703.764 & 273.873 & 150.840 & 40.746 \\
\hline 383.747 & 5040.889 & 202.794 & -170.967 & 139.963 \\
\hline 127.363 & 4724.546 & 264.358 & 72.288 & 20.204 \\
\hline 756.290 & 5022.854 & 81.863 & -2386.617 & 675.423 \\
\hline 438.726 & 4948.178 & 115.356 & 99.417 & 75.963 \\
\hline 394.234 & 4965.520 & 156.489 & 90.243 & 146.224 \\
\hline 391.511 & 4975.818 & 131.730 & 21.055 & 81.868 \\
\hline 180.280 & 4669.830 & 501.085 & 106.850 & 46.083 \\
\hline 136.208 & 4841.019 & 294.559 & 53.943 & 30.307 \\
\hline 157.554 & 4831.531 & 250.905 & 58.268 & 26.375 \\
\hline 282.254 & 4974.107 & 83.901 & 77.135 & 110.125 \\
\hline 115.865 & 4730.719 & 141.922 & 264.918 & 39.298 \\
\hline 185.660 & 4635.019 & 318.436 & 210.793 & 50.081 \\
\hline 305.638 & 4935.904 & 109.958 & 244.266 & 109.290 \\
\hline 171.202 & 4765.170 & 312.361 & 215.893 & 80.750 \\
\hline 342.103 & 4517.650 & 149.591 & 264.956 & 36.371 \\
\hline 147.198 & 4601.546 & 287.597 & 178.259 & 35.503 \\
\hline 84.304 & 4478.622 & 76.167 & 241.593 & 13.545 \\
\hline 254.063 & 4632.813 & 212.514 & 76.735 & 15.731 \\
\hline 135.027 & 4747.041 & 214.556 & 118.729 & 29.155 \\
\hline 112.630 & 4433.327 & 147.778 & 262.229 & 20.956 \\
\hline 327.804 & 4955.142 & 163.356 & 99.869 & 121.045 \\
\hline 454.160 & 4999.543 & 124.249 & -145.643 & 209.060 \\
\hline 435.457 & 4842.908 & 428.633 & 176.853 & 132.439 \\
\hline 149.093 & 4863.519 & 449.622 & 38.718 & 39.474 \\
\hline 142.801 & 4712.115 & 211.397 & 112.256 & 23.954 \\
\hline 133.096 & 4848.561 & 510.375 & 27.571 & 28.767 \\
\hline 226.222 & 4857.929 & 247.659 & 97.968 & 50.963 \\
\hline 1122.010 & 4693.251 & 653.752 & 28.318 & 17.458 \\
\hline 155.963 & 4730.719 & 743.399 & 40.062 & 32.062 \\
\hline 120.783 & 4783.058 & 283.294 & 66.829 & 25.700 \\
\hline 206.730 & 4850.440 & 376.052 & 31.782 & 29.122 \\
\hline 214.759 & 4956.877 & 181.349 & 31.240 & 55.053 \\
\hline 264.534 & 4996.180 & 88.900 & 0.000 & 450.088 \\
\hline 168.279 & 4104.897 & 162.035 & 225.241 & 18.392 \\
\hline
\end{tabular}




$\begin{array}{lcccc}199.673 & 4578.760 & 210.768 & 212.892 & 30.889 \\ 163.283 & 4488.485 & 136.474 & 237.812 & 21.239 \\ 122.750 & 4435.883 & 147.604 & 271.344 & 22.537 \\ 205.225 & 4610.554 & 93.070 & 162.493 & 17.230 \\ 216.052 & 4632.813 & 361.274 & 177.273 & 47.571 \\ 161.356 & 4619.502 & 463.376 & 111.097 & 37.748 \\ 118.479 & 4389.109 & 396.845 & 127.704 & 22.683 \\ 183.197 & 4857.929 & 203.208 & 154.166 & 62.917 \\ 187.683 & 4821.975 & 297.334 & 113.363 & 54.581\end{array}$


Page 121 of 203

Page 121 of 203 


\begin{tabular}{|c|c|c|c|c|c|c|}
\hline Sample & ${ }^{207} \mathrm{~Pb} /{ }^{235} \mathrm{U}$ & Error $2 \sigma$ & ${ }^{206} \mathrm{~Pb} /{ }^{238} \mathrm{U}$ & Error $2 \sigma$ & \multicolumn{2}{|c|}{ rrelation_6_3 $3 \varepsilon^{238} \mathrm{U} /{ }^{206} \mathrm{~Pb}$} \\
\hline PCA_1 & 5.420 & 0.290 & 0.349 & 0.014 & 0.462 & 2.865 \\
\hline PCA_2 & 5.910 & 0.270 & 0.351 & 0.012 & 0.349 & 2.849 \\
\hline PCA_3 & 5.570 & 0.300 & 0.336 & 0.014 & 0.390 & 2.976 \\
\hline PCA_4 & 5.570 & 0.290 & 0.351 & 0.012 & 0.424 & 2.849 \\
\hline PCA_5 & 5.980 & 0.280 & 0.356 & 0.016 & 0.451 & 2.809 \\
\hline PCA_6 & 6.170 & 0.370 & 0.362 & 0.011 & 0.372 & 2.762 \\
\hline PCA_7 & 5.690 & 0.320 & 0.335 & 0.011 & 0.365 & 2.985 \\
\hline PCA_8 & 5.470 & 0.300 & 0.356 & 0.012 & 0.233 & 2.809 \\
\hline PCA_9 & 6.050 & 0.310 & 0.361 & 0.014 & 0.493 & 2.770 \\
\hline PCA_10 & 5.480 & 0.290 & 0.344 & 0.012 & 0.516 & 2.907 \\
\hline PCA_11 & 5.520 & 0.290 & 0.362 & 0.014 & 0.390 & 2.762 \\
\hline PCA_12 & 7.900 & 0.680 & 0.372 & 0.014 & 0.566 & 2.688 \\
\hline PCA_13 & 5.340 & 0.290 & 0.356 & 0.014 & 0.431 & 2.809 \\
\hline PCA_14 & 5.070 & 0.310 & 0.306 & 0.010 & 0.381 & 3.268 \\
\hline PCA_15 & 4.950 & 0.310 & 0.305 & 0.011 & 0.313 & 3.279 \\
\hline PCA_16 & 5.470 & 0.240 & 0.344 & 0.010 & 0.253 & 2.905 \\
\hline PCA_17 & 5.420 & 0.310 & 0.349 & 0.012 & 0.391 & 2.865 \\
\hline PCA_18 & 5.720 & 0.310 & 0.357 & 0.014 & 0.344 & 2.801 \\
\hline PCA_19 & 5.690 & 0.280 & 0.355 & 0.011 & 0.271 & 2.817 \\
\hline PCA_20 & 5.320 & 0.280 & 0.329 & 0.011 & 0.211 & 3.040 \\
\hline PCA_21 & 5.200 & 0.310 & 0.342 & 0.013 & 0.505 & 2.924 \\
\hline PCA_22 & 5.320 & 0.270 & 0.345 & 0.011 & 0.186 & 2.899 \\
\hline PCA_23 & 5.470 & 0.260 & 0.345 & 0.012 & 0.375 & 2.899 \\
\hline PCA_24 & 5.670 & 0.340 & 0.365 & 0.014 & 0.565 & 2.740 \\
\hline PCA_25 & 5.590 & 0.260 & 0.342 & 0.010 & 0.340 & 2.924 \\
\hline PCA_26 & 6.480 & 0.440 & 0.363 & 0.016 & 0.705 & 2.755 \\
\hline PCA_27 & 5.620 & 0.260 & 0.368 & 0.013 & 0.309 & 2.717 \\
\hline PCA_28 & 5.700 & 0.300 & 0.356 & 0.015 & 0.489 & 2.809 \\
\hline PCA_29 & 5.210 & 0.240 & 0.332 & 0.012 & 0.478 & 3.012 \\
\hline R_R19_1 & 0.620 & 0.110 & 0.076 & 0.004 & 0.290 & 13.141 \\
\hline R_R19_2 & 0.720 & 0.110 & 0.084 & 0.005 & 0.010 & 11.933 \\
\hline R_R19_3 & 0.561 & 0.094 & 0.078 & 0.005 & 0.221 & 12.837 \\
\hline R_R19_4 & 0.645 & 0.098 & 0.082 & 0.005 & 0.188 & 12.255 \\
\hline R_R19_5 & 0.710 & 0.100 & 0.082 & 0.004 & 0.164 & 12.136 \\
\hline R_R19_6 & 0.760 & 0.110 & 0.084 & 0.004 & 0.290 & 11.848 \\
\hline R_R19_7 & 0.723 & 0.093 & 0.081 & 0.005 & 0.076 & 12.285 \\
\hline R_R19_8 & 0.620 & 0.100 & 0.082 & 0.005 & 0.010 & 12.136 \\
\hline R_R19_9 & 0.557 & 0.076 & 0.080 & 0.005 & 0.010 & 12.563 \\
\hline R_R19_10 & 0.715 & 0.088 & 0.086 & 0.005 & 0.261 & 11.682 \\
\hline R_R19_11 & 0.608 & 0.098 & 0.077 & 0.005 & 0.122 & 13.004 \\
\hline R_R19_12 & 0.595 & 0.091 & 0.073 & 0.003 & 0.363 & 13.717 \\
\hline R_R19_13 & 0.670 & 0.110 & 0.081 & 0.005 & 0.027 & 12.346 \\
\hline R_R19_14 & 0.670 & 0.096 & 0.083 & 0.004 & 0.218 & 11.990 \\
\hline R_R19_15 & 0.631 & 0.078 & 0.079 & 0.005 & 0.125 & 12.594 \\
\hline R_R19_16 & 0.761 & 0.096 & 0.080 & 0.005 & 0.143 & 12.453 \\
\hline R_R19_17 & 0.474 & 0.079 & 0.075 & 0.004 & 0.188 & 13.263 \\
\hline R_R19_18 & 0.557 & 0.076 & 0.077 & 0.004 & 0.091 & 12.953 \\
\hline R_R19_19 & 0.690 & 0.110 & 0.091 & 0.004 & 0.265 & 10.989 \\
\hline
\end{tabular}




\begin{tabular}{|c|c|c|c|c|c|c|}
\hline R_R19_20 & 0.650 & 0.110 & 0.079 & 0.004 & 0.117 & 12.723 \\
\hline R_R19_21 & 0.643 & 0.092 & 0.079 & 0.004 & 0.105 & 12.626 \\
\hline R_R19_22 & 0.569 & 0.076 & 0.070 & 0.004 & 0.212 & 14.306 \\
\hline R_R19_23 & 0.546 & 0.090 & 0.076 & 0.004 & 0.222 & 13.123 \\
\hline R_R19_24 & 0.682 & 0.084 & 0.083 & 0.004 & 0.216 & 12.077 \\
\hline R_R19_25 & 0.578 & 0.083 & 0.084 & 0.005 & 0.270 & 11.933 \\
\hline R_R19_26 & 0.493 & 0.080 & 0.062 & 0.004 & 0.198 & 16.181 \\
\hline R_R19_27 & 0.462 & 0.075 & 0.070 & 0.005 & 0.145 & 14.306 \\
\hline R_R19_28 & 0.590 & 0.100 & 0.076 & 0.005 & 0.430 & 13.245 \\
\hline R_R19_29 & 0.462 & 0.074 & 0.073 & 0.005 & 0.158 & 13.624 \\
\hline R632_1 & 0.645 & 0.022 & 0.081 & 0.002 & 0.345 & 12.407 \\
\hline R632_2 & 0.653 & 0.022 & 0.083 & 0.001 & 0.384 & 12.077 \\
\hline R632_3 & 0.658 & 0.022 & 0.080 & 0.001 & 0.270 & 12.563 \\
\hline R632_4 & 0.634 & 0.023 & 0.081 & 0.002 & 0.614 & 12.346 \\
\hline R632_5 & 0.620 & 0.019 & 0.079 & 0.001 & 0.416 & 12.594 \\
\hline R632_6 & 0.671 & 0.016 & 0.084 & 0.002 & 0.353 & 11.862 \\
\hline R632_7 & 0.636 & 0.019 & 0.080 & 0.002 & 0.489 & 12.531 \\
\hline R632_8 & 0.631 & 0.023 & 0.081 & 0.002 & 0.398 & 12.285 \\
\hline R632_9 & 0.669 & 0.027 & 0.083 & 0.002 & 0.381 & 11.990 \\
\hline R632_10 & 0.646 & 0.029 & 0.080 & 0.002 & 0.499 & 12.500 \\
\hline R632_11 & 0.611 & 0.021 & 0.077 & 0.002 & 0.209 & 12.970 \\
\hline R632_12 & 0.631 & 0.022 & 0.080 & 0.002 & 0.484 & 12.547 \\
\hline R632_13 & 0.670 & 0.020 & 0.085 & 0.002 & 0.306 & 11.751 \\
\hline R632_14 & 0.668 & 0.020 & 0.083 & 0.002 & 0.545 & 12.063 \\
\hline R632_15 & 0.596 & 0.018 & 0.076 & 0.002 & 0.140 & 13.141 \\
\hline R632_16 & 0.678 & 0.023 & 0.084 & 0.002 & 0.270 & 11.905 \\
\hline R632_17 & 0.628 & 0.022 & 0.083 & 0.002 & 0.442 & 12.092 \\
\hline R632_18 & 0.658 & 0.024 & 0.082 & 0.002 & 0.354 & 12.195 \\
\hline R632_19 & 0.639 & 0.023 & 0.082 & 0.002 & 0.558 & 12.255 \\
\hline R632_20 & 0.632 & 0.023 & 0.080 & 0.002 & 0.385 & 12.484 \\
\hline R632_21 & 0.638 & 0.023 & 0.080 & 0.002 & 0.202 & 12.531 \\
\hline R632_22 & 0.653 & 0.022 & 0.083 & 0.002 & 0.215 & 11.990 \\
\hline R632_23 & 0.573 & 0.022 & 0.072 & 0.002 & 0.531 & 13.850 \\
\hline R632_24 & 0.700 & 0.019 & 0.089 & 0.002 & 0.624 & 11.249 \\
\hline R632_25 & 0.630 & 0.018 & 0.078 & 0.002 & 0.329 & 12.870 \\
\hline$R 632$ 26 & 0.578 & 0.020 & 0.070 & 0.002 & 0.475 & 14.265 \\
\hline$R 632$ R27 & 0.575 & 0.022 & 0.074 & 0.002 & 0.614 & 13.605 \\
\hline R632_28 & 0.680 & 0.020 & 0.083 & 0.002 & 0.552 & 12.019 \\
\hline R632_29 & 0.685 & 0.019 & 0.086 & 0.002 & 0.365 & 11.641 \\
\hline X11B_1 & 55.100 & 3.000 & 0.476 & 0.025 & 0.758 & 2.101 \\
\hline X11B_2 & 84.100 & 6.400 & 0.725 & 0.064 & 0.954 & 1.379 \\
\hline X11B_3 & 15.600 & 2.700 & 0.153 & 0.026 & 0.870 & 6.536 \\
\hline X11B_4 & 19.700 & 2.700 & 0.188 & 0.022 & 0.937 & 5.319 \\
\hline X11B_5 & 18.580 & 0.990 & 0.189 & 0.009 & 0.752 & 5.291 \\
\hline X11B_6 & 20.780 & 0.790 & 0.215 & 0.008 & 0.472 & 4.647 \\
\hline X11B_7 & 5.270 & 0.560 & 0.057 & 0.005 & 0.764 & 17.422 \\
\hline X11B_8 & 18.100 & 1.200 & 0.165 & 0.010 & 0.686 & 6.075 \\
\hline X11B_9 & 13.200 & 1.300 & 0.124 & 0.012 & 0.866 & 8.065 \\
\hline
\end{tabular}




\begin{tabular}{|c|c|c|c|c|c|c|}
\hline X11B_10 & 41.400 & 1.700 & 0.368 & 0.016 & 0.881 & 2.717 \\
\hline X11B_11 & 49.800 & 3.700 & 0.452 & 0.031 & 0.885 & 2.212 \\
\hline X11B_12 & 11.750 & 0.600 & 0.145 & 0.007 & 0.870 & 6.916 \\
\hline X11B_13 & 13.840 & 0.440 & 0.164 & 0.005 & 0.454 & 6.098 \\
\hline X11B_14 & 30.650 & 0.900 & 0.277 & 0.008 & 0.571 & 3.613 \\
\hline X11B_15 & 52.100 & 1.900 & 0.463 & 0.023 & 0.827 & 2.160 \\
\hline X11B_16 & 22.300 & 1.700 & 0.217 & 0.016 & 0.828 & 4.608 \\
\hline X93_1 & 108.600 & 7.500 & 0.910 & 0.058 & 0.966 & 1.099 \\
\hline X93_2 & 138.800 & 6.800 & 1.204 & 0.065 & 0.912 & 0.831 \\
\hline X93_3 & 273.000 & 10.000 & 2.381 & 0.092 & 0.882 & 0.420 \\
\hline X93_4 & 360.000 & 18.000 & 3.060 & 0.140 & 0.907 & 0.327 \\
\hline X93_5 & 790.000 & 130.000 & 6.900 & 1.100 & 0.980 & 0.145 \\
\hline X93_6 & 1620.000 & 470.000 & 13.900 & 4.000 & 0.995 & 0.072 \\
\hline X93_7 & 272.000 & 26.000 & 2.310 & 0.210 & 0.911 & 0.433 \\
\hline X93_8 & 1200.000 & 340.000 & 10.900 & 3.300 & 0.911 & 0.092 \\
\hline X93_9 & 650.000 & 120.000 & 5.500 & 1.000 & 0.985 & 0.182 \\
\hline X93_10 & 1170.000 & 200.000 & 10.000 & 1.700 & 0.986 & 0.100 \\
\hline X93_11 & 245.000 & 24.000 & 2.270 & 0.220 & 0.952 & 0.441 \\
\hline X93_12 & 312.000 & 23.000 & 2.700 & 0.190 & 0.915 & 0.370 \\
\hline X93_13 & 236.000 & 17.000 & 2.070 & 0.160 & 0.927 & 0.483 \\
\hline X93_14 & 255.000 & 18.000 & 2.200 & 0.150 & 0.914 & 0.455 \\
\hline X93_15 & 214.000 & 18.000 & 1.840 & 0.150 & 0.936 & 0.543 \\
\hline X93_16 & 287.000 & 17.000 & 2.560 & 0.170 & 0.913 & 0.391 \\
\hline X93_17 & 82.000 & 13.000 & 0.760 & 0.120 & 0.961 & 1.316 \\
\hline X93_18 & 800.000 & 210.000 & 7.000 & 1.800 & 0.991 & 0.143 \\
\hline X93_19 & 437.000 & 40.000 & 3.840 & 0.350 & 0.934 & 0.260 \\
\hline X93_20 & 440.000 & 65.000 & 4.040 & 0.650 & 0.950 & 0.248 \\
\hline X93_21 & 226.000 & 20.000 & 1.910 & 0.170 & 0.962 & 0.524 \\
\hline X93_22 & 560.000 & 87.000 & 4.660 & 0.680 & 0.979 & 0.215 \\
\hline X93_23 & 1410.000 & 380.000 & 12.500 & 3.400 & 0.993 & 0.080 \\
\hline X93_24 & 192.000 & 15.000 & 1.700 & 0.140 & 0.872 & 0.588 \\
\hline X93_25 & 225.000 & 15.000 & 1.870 & 0.130 & 0.856 & 0.535 \\
\hline X93_26 & 920.000 & 110.000 & 7.900 & 1.000 & 0.981 & 0.127 \\
\hline X93_27 & 316.000 & 32.000 & 2.690 & 0.290 & 0.934 & 0.372 \\
\hline X93_28 & 78.400 & 5.600 & 0.686 & 0.040 & 0.479 & 1.458 \\
\hline X93_29 & 238.000 & 28.000 & 2.120 & 0.270 & 0.950 & 0.472 \\
\hline X93_30 & 69.400 & 6.500 & 0.623 & 0.050 & 0.876 & 1.605 \\
\hline X93_31 & 74.100 & 8.300 & 0.656 & 0.071 & 0.934 & 1.524 \\
\hline X93_32 & 173.000 & 15.000 & 1.550 & 0.140 & 0.961 & 0.645 \\
\hline X93_33 & 89.000 & 16.000 & 0.790 & 0.140 & 0.947 & 1.266 \\
\hline X93_34 & 148.000 & 15.000 & 1.300 & 0.120 & 0.952 & 0.769 \\
\hline X93_35 & 131.000 & 11.000 & 1.160 & 0.110 & 0.953 & 0.862 \\
\hline X93_36 & 384.000 & 28.000 & 3.330 & 0.280 & 0.878 & 0.300 \\
\hline X93_37 & 532.000 & 39.000 & 4.710 & 0.360 & 0.900 & 0.212 \\
\hline X93_38 & 970.000 & 130.000 & 8.200 & 1.100 & 0.974 & 0.122 \\
\hline X93_39 & 3230.000 & 630.000 & 28.200 & 5.300 & 0.989 & 0.035 \\
\hline X93_40 & 970.000 & 190.000 & 8.500 & 1.700 & 0.989 & 0.118 \\
\hline X93_41 & 183.000 & 14.000 & 1.650 & 0.130 & 0.807 & 0.606 \\
\hline X93_42 & 156.000 & 16.000 & 1.370 & 0.120 & 0.879 & 0.730 \\
\hline
\end{tabular}




\begin{tabular}{|c|c|c|c|c|c|c|}
\hline X93_43 & 55.700 & 5.400 & 0.488 & 0.040 & 0.841 & 2.049 \\
\hline X93_44 & 279.000 & 31.000 & 2.410 & 0.300 & 0.927 & 0.415 \\
\hline X93_45 & 2710.000 & 410.000 & 22.700 & 3.300 & 0.993 & 0.044 \\
\hline X93_46 & 399.000 & 39.000 & 3.430 & 0.340 & 0.920 & 0.292 \\
\hline X93_47 & 161.000 & 18.000 & 1.360 & 0.150 & 0.876 & 0.735 \\
\hline X93_48 & 151.000 & 44.000 & 1.200 & 0.240 & 0.978 & 0.833 \\
\hline X93_49 & 111.200 & 7.900 & 0.979 & 0.064 & 0.885 & 1.021 \\
\hline X93_50 & 273.000 & 23.000 & 2.290 & 0.170 & 0.850 & 0.437 \\
\hline X93_51 & 2500.000 & 780.000 & 19.300 & 5.700 & 0.989 & 0.052 \\
\hline X93_52 & 262.000 & 56.000 & 2.160 & 0.470 & 0.981 & 0.463 \\
\hline X93_53 & 212.000 & 22.000 & 1.910 & 0.200 & 0.902 & 0.524 \\
\hline X93_54 & 1620.000 & 220.000 & 14.200 & 1.900 & 0.991 & 0.070 \\
\hline X93_55 & 130.000 & 11.000 & 1.104 & 0.088 & 0.890 & 0.906 \\
\hline X93_56 & 320.000 & 32.000 & 2.710 & 0.280 & 0.890 & 0.369 \\
\hline X93_57 & 66.700 & 5.300 & 0.600 & 0.045 & 0.851 & 1.667 \\
\hline X96_1 & 590.000 & 120.000 & 5.100 & 1.100 & 0.989 & 0.196 \\
\hline X96_2 & 316.000 & 35.000 & 2.620 & 0.280 & 0.985 & 0.382 \\
\hline X96_3 & 415.000 & 23.000 & 3.610 & 0.220 & 0.955 & 0.277 \\
\hline X96_4 & 165.000 & 6.800 & 1.407 & 0.061 & 0.889 & 0.711 \\
\hline X96_5 & 2380.000 & 280.000 & 21.200 & 2.700 & 0.984 & 0.047 \\
\hline X96_6 & 304.000 & 31.000 & 2.570 & 0.260 & 0.985 & 0.389 \\
\hline X96_7 & 323.000 & 18.000 & 2.780 & 0.160 & 0.936 & 0.360 \\
\hline X96_8 & 1278.000 & 72.000 & 10.770 & 0.600 & 0.973 & 0.093 \\
\hline X96_9 & 136.000 & 13.000 & 1.150 & 0.100 & 0.946 & 0.870 \\
\hline X96_10 & 5.240 & 0.360 & 0.050 & 0.003 & 0.955 & 19.881 \\
\hline X96_11 & 197.000 & 13.000 & 1.670 & 0.110 & 0.939 & 0.599 \\
\hline X96_12 & 262.000 & 35.000 & 2.320 & 0.320 & 0.982 & 0.431 \\
\hline X96_13 & 45.700 & 4.900 & 0.412 & 0.047 & 0.917 & 2.427 \\
\hline X96_14 & 157.900 & 8.500 & 1.356 & 0.078 & 0.925 & 0.737 \\
\hline X96_15 & 1405.000 & 77.000 & 12.050 & 0.740 & 0.926 & 0.083 \\
\hline X96_16 & 314.000 & 20.000 & 2.690 & 0.180 & 0.961 & 0.372 \\
\hline X96_17 & 147.000 & 5.700 & 1.299 & 0.053 & 0.918 & 0.770 \\
\hline X96_18 & 602.000 & 24.000 & 5.140 & 0.190 & 0.952 & 0.195 \\
\hline X96_19 & 5750.000 & 740.000 & 50.200 & 6.400 & 0.991 & 0.020 \\
\hline X96_20 & 439.000 & 38.000 & 3.790 & 0.320 & 0.965 & 0.264 \\
\hline X96_21 & 234.000 & 40.000 & 2.030 & 0.330 & 0.985 & 0.493 \\
\hline X96_22 & 1470.000 & 320.000 & 12.400 & 2.700 & 0.995 & 0.081 \\
\hline X96_23 & 461.000 & 33.000 & 3.960 & 0.280 & 0.975 & 0.253 \\
\hline X96_24 & 299.000 & 17.000 & 2.660 & 0.150 & 0.943 & 0.376 \\
\hline X96_25 & 185.000 & 43.000 & 1.620 & 0.390 & 0.991 & 0.617 \\
\hline X96_26 & 107.500 & 9.500 & 0.962 & 0.092 & 0.940 & 1.040 \\
\hline X96_27 & 339.000 & 30.000 & 3.020 & 0.290 & 0.947 & 0.331 \\
\hline X96_30 & 920.000 & 320.000 & 6.900 & 1.900 & 0.998 & 0.145 \\
\hline X96_31 & 451.000 & 25.000 & 3.780 & 0.210 & 0.916 & 0.265 \\
\hline X96_32 & 153.000 & 22.000 & 1.300 & 0.180 & 0.981 & 0.769 \\
\hline X96_33 & 326.000 & 17.000 & 2.890 & 0.160 & 0.918 & 0.346 \\
\hline X96_34 & 337.000 & 24.000 & 2.890 & 0.200 & 0.967 & 0.346 \\
\hline X96_35 & 1520.000 & 130.000 & 13.000 & 1.100 & 0.978 & 0.077 \\
\hline X96_36 & 1620.000 & 120.000 & 13.740 & 0.960 & 0.980 & 0.073 \\
\hline
\end{tabular}




\begin{tabular}{|c|c|c|c|c|c|c|}
\hline X96_37 & 700.000 & 46.000 & 6.020 & 0.390 & 0.977 & 0.166 \\
\hline X96_38 & 1750.000 & 250.000 & 14.600 & 2.100 & 0.995 & 0.068 \\
\hline X96_39 & 3060.000 & 450.000 & 26.100 & 3.700 & 0.984 & 0.038 \\
\hline X96_40 & 81.800 & 6.700 & 0.682 & 0.060 & 0.763 & 1.466 \\
\hline X96_41 & 32.300 & 3.100 & 0.301 & 0.035 & 0.888 & 3.322 \\
\hline X96_42 & 3600.000 & 1400.000 & 30.000 & 12.000 & 0.999 & 0.033 \\
\hline X96_43 & 565.000 & 30.000 & 4.900 & 0.270 & 0.926 & 0.204 \\
\hline X96_44 & 495.000 & 42.000 & 4.040 & 0.340 & 0.929 & 0.248 \\
\hline X96_45 & 630.000 & 59.000 & 5.450 & 0.510 & 0.979 & 0.183 \\
\hline X96_46 & 222.000 & 31.000 & 1.950 & 0.260 & 0.990 & 0.513 \\
\hline X96_47 & 108.000 & 18.000 & 0.980 & 0.170 & 0.994 & 1.020 \\
\hline X96_48 & 505.000 & 52.000 & 4.360 & 0.450 & 0.985 & 0.229 \\
\hline X96_49 & 2450.000 & 280.000 & 20.900 & 2.400 & 0.987 & 0.048 \\
\hline X96_50 & 150.000 & 22.000 & 1.320 & 0.200 & 0.980 & 0.758 \\
\hline x96_51 & 275.000 & 19.000 & 2.280 & 0.170 & 0.955 & 0.439 \\
\hline x96_52 & 92.000 & 8.100 & 0.799 & 0.071 & 0.917 & 1.252 \\
\hline X96_53 & 940.000 & 190.000 & 8.500 & 1.800 & 0.994 & 0.118 \\
\hline X96_54 & 112.000 & 17.000 & 0.880 & 0.130 & 0.966 & 1.136 \\
\hline X96_55 & 1510.000 & 110.000 & 12.610 & 0.900 & 0.962 & 0.079 \\
\hline X96_56 & 200.000 & 11.000 & 1.725 & 0.091 & 0.964 & 0.580 \\
\hline X96_57 & 172.400 & 5.800 & 1.482 & 0.053 & 0.854 & 0.675 \\
\hline x96_58 & 1019.000 & 97.000 & 8.650 & 0.790 & 0.983 & 0.116 \\
\hline X96_59 & 144.000 & 11.000 & 1.268 & 0.096 & 0.932 & 0.789 \\
\hline x96_60 & 46.500 & 3.100 & 0.401 & 0.026 & 0.845 & 2.494 \\
\hline x96_61 & 73.300 & 3.300 & 0.636 & 0.028 & 0.852 & 1.572 \\
\hline x96_62 & 16.700 & 2.000 & 0.155 & 0.019 & 0.885 & 6.452 \\
\hline x96_63 & 66.100 & 4.500 & 0.571 & 0.039 & 0.915 & 1.751 \\
\hline x96_64 & 292.000 & 16.000 & 2.430 & 0.150 & 0.950 & 0.412 \\
\hline x96_65 & 217.000 & 21.000 & 1.850 & 0.180 & 0.931 & 0.541 \\
\hline x96_66 & 2220.000 & 310.000 & 18.900 & 2.600 & 0.993 & 0.053 \\
\hline x96_67 & 86.000 & 6.600 & 0.759 & 0.056 & 0.907 & 1.318 \\
\hline x96_68 & 124.000 & 26.000 & 1.070 & 0.210 & 0.996 & 0.935 \\
\hline x96_69 & 247.000 & 22.000 & 2.150 & 0.180 & 0.827 & 0.465 \\
\hline x96_70 & 730.000 & 120.000 & 6.300 & 1.100 & 0.964 & 0.159 \\
\hline x96_71 & 154.000 & 12.000 & 1.350 & 0.110 & 0.960 & 0.741 \\
\hline x96_72 & 63.100 & 3.500 & 0.565 & 0.028 & 0.764 & 1.770 \\
\hline x96_73 & 862.000 & 79.000 & 7.260 & 0.640 & 0.974 & 0.138 \\
\hline X96_74 & 318.000 & 42.000 & 2.750 & 0.340 & 0.979 & 0.364 \\
\hline X96_75 & 1007.000 & 84.000 & 8.440 & 0.680 & 0.966 & 0.118 \\
\hline x96_76 & 203.000 & 24.000 & 1.710 & 0.180 & 0.973 & 0.585 \\
\hline X96_77 & 361.000 & 58.000 & 3.200 & 0.510 & 0.985 & 0.313 \\
\hline x96_78 & 87.000 & 12.000 & 0.780 & 0.110 & 0.972 & 1.282 \\
\hline x96_79 & 173.000 & 16.000 & 1.480 & 0.140 & 0.962 & 0.676 \\
\hline x96_80 & 116.000 & 11.000 & 0.990 & 0.081 & 0.964 & 1.010 \\
\hline X96_81 & 540.000 & 100.000 & 4.390 & 0.830 & 0.995 & 0.228 \\
\hline X96_82 & 1062.000 & 80.000 & 9.320 & 0.700 & 0.970 & 0.107 \\
\hline X96_83 & 64.000 & 11.000 & 0.554 & 0.095 & 0.991 & 1.805 \\
\hline X96_84 & 7.400 & 1.300 & 0.070 & 0.013 & 0.990 & 14.286 \\
\hline X96_85 & 95.600 & 4.600 & 0.836 & 0.037 & 0.856 & 1.196 \\
\hline X96_86 & 480.000 & 51.000 & 4.220 & 0.440 & 0.937 & 0.237 \\
\hline
\end{tabular}




$\begin{array}{lllllll}\text { X96_87 } & 360.000 & 200.000 & 3.000 & 1.700 & 0.987 & 0.333 \\ \text { X96_88 } & 171.000 & 14.000 & 1.460 & 0.120 & 0.958 & 0.685 \\ \text { X96_89 } & 319.000 & 17.000 & 2.800 & 0.150 & 0.930 & 0.357 \\ \text { X96_90 } & 151.000 & 23.000 & 1.260 & 0.190 & 0.975 & 0.794 \\ \text { X96_91 } & 100.000 & 15.000 & 0.840 & 0.120 & 0.991 & 1.190 \\ \text { X96_92 } & 194.000 & 12.000 & 1.650 & 0.110 & 0.961 & 0.606 \\ \text { X96_93 } & 273.000 & 16.000 & 2.330 & 0.140 & 0.932 & 0.429 \\ \text { X96_94 } & 204.000 & 11.000 & 1.752 & 0.086 & 0.948 & 0.571 \\ \text { X96_95 } & 178.900 & 8.300 & 1.527 & 0.074 & 0.956 & 0.655 \\ \text { X96_96 } & 254.000 & 17.000 & 2.220 & 0.140 & 0.951 & 0.450\end{array}$




\begin{tabular}{|c|c|c|c|c|c|c|}
\hline Error $2 \sigma$ & ${ }^{207} \mathrm{~Pb} /{ }^{206} \mathrm{~Pb}$ & Error $2 \sigma$ & Jrrelation_38 & alAge ${ }^{206} \mathrm{~Pb} /{ }^{23}$ & Error $2 \sigma$ & IalAge ${ }^{207} \mathrm{~Pb} /{ }^{23}$ \\
\hline 0.115 & 0.112 & 0.005 & 0.329 & 1929.822 & 77.414 & 1888.022 \\
\hline 0.097 & 0.121 & 0.007 & 0.321 & 1939.372 & 66.303 & 1962.705 \\
\hline 0.124 & 0.118 & 0.007 & 0.383 & 1867.398 & 77.808 & 1911.473 \\
\hline 0.097 & 0.117 & 0.006 & 0.307 & 1939.372 & 66.303 & 1911.473 \\
\hline 0.126 & 0.123 & 0.006 & 0.290 & 1963.186 & 88.233 & 1972.939 \\
\hline 0.084 & 0.118 & 0.007 & 0.188 & 1991.647 & 60.520 & 2000.209 \\
\hline 0.098 & 0.123 & 0.007 & 0.325 & 1862.571 & 61.159 & 1929.851 \\
\hline 0.095 & 0.112 & 0.007 & 0.357 & 1963.186 & 66.175 & 1895.899 \\
\hline 0.107 & 0.122 & 0.006 & 0.251 & 1986.912 & 77.055 & 1983.071 \\
\hline 0.101 & 0.118 & 0.006 & 0.255 & 1905.884 & 66.484 & 1897.467 \\
\hline 0.107 & 0.113 & 0.006 & 0.331 & 1991.647 & 77.025 & 1903.716 \\
\hline 0.101 & 0.153 & 0.012 & -0.133 & 2038.804 & 76.729 & 2219.679 \\
\hline 0.110 & 0.113 & 0.006 & 0.333 & 1963.186 & 77.204 & 1875.289 \\
\hline 0.107 & 0.116 & 0.007 & 0.260 & 1720.993 & 56.242 & 1831.100 \\
\hline 0.118 & 0.116 & 0.007 & 0.327 & 1716.055 & 61.891 & 1810.825 \\
\hline 0.081 & 0.114 & 0.005 & 0.311 & 1906.843 & 53.183 & 1895.899 \\
\hline 0.099 & 0.115 & 0.006 & 0.262 & 1929.822 & 66.355 & 1888.022 \\
\hline 0.110 & 0.117 & 0.006 & 0.398 & 1967.938 & 77.174 & 1934.394 \\
\hline 0.087 & 0.120 & 0.006 & 0.280 & 1958.430 & 60.684 & 1929.851 \\
\hline 0.102 & 0.118 & 0.007 & 0.400 & 1833.533 & 61.304 & 1872.081 \\
\hline 0.111 & 0.111 & 0.006 & 0.194 & 1896.284 & 72.081 & 1852.616 \\
\hline 0.092 & 0.112 & 0.006 & 0.375 & 1910.679 & 60.920 & 1872.081 \\
\hline 0.101 & 0.117 & 0.006 & 0.404 & 1910.679 & 66.458 & 1895.899 \\
\hline 0.105 & 0.113 & 0.006 & 0.015 & 2005.830 & 76.936 & 1926.811 \\
\hline 0.085 & 0.118 & 0.006 & 0.356 & 1896.284 & 55.447 & 1914.559 \\
\hline 0.121 & 0.129 & 0.006 & -0.165 & 1996.378 & 87.995 & 2043.187 \\
\hline 0.096 & 0.109 & 0.005 & 0.342 & 2019.983 & 71.358 & 1919.171 \\
\hline 0.118 & 0.115 & 0.005 & 0.433 & 1963.186 & 82.719 & 1931.368 \\
\hline 0.109 & 0.118 & 0.005 & 0.285 & 1848.068 & 66.798 & 1854.253 \\
\hline 0.691 & 0.053 & 0.009 & -0.009 & 472.802 & 24.852 & 489.847 \\
\hline 0.712 & 0.064 & 0.010 & 0.264 & 518.765 & 30.953 & 550.667 \\
\hline 0.791 & 0.055 & 0.009 & 0.207 & 483.576 & 29.797 & 452.177 \\
\hline 0.781 & 0.057 & 0.009 & 0.403 & 505.666 & 32.224 & 505.397 \\
\hline 0.604 & 0.062 & 0.009 & 0.238 & 510.432 & 25.398 & 544.746 \\
\hline 0.547 & 0.068 & 0.010 & -0.064 & 522.333 & 24.136 & 574.010 \\
\hline 0.679 & 0.064 & 0.009 & 0.378 & 504.474 & 27.889 & 552.436 \\
\hline 0.766 & 0.057 & 0.009 & 0.256 & 510.432 & 32.212 & 489.847 \\
\hline 0.726 & 0.053 & 0.008 & 0.231 & 493.735 & 28.532 & 449.572 \\
\hline 0.641 & 0.061 & 0.007 & 0.201 & 529.462 & 29.071 & 547.711 \\
\hline 0.795 & 0.062 & 0.011 & 0.227 & 477.593 & 29.190 & 482.298 \\
\hline 0.640 & 0.058 & 0.008 & -0.023 & 453.604 & 21.156 & 474.056 \\
\hline 0.716 & 0.062 & 0.010 & 0.375 & 502.089 & 29.134 & 520.712 \\
\hline 0.532 & 0.055 & 0.008 & 0.047 & 516.385 & 22.909 & 520.712 \\
\hline 0.746 & 0.061 & 0.009 & 0.175 & 492.540 & 29.155 & 496.719 \\
\hline 0.775 & 0.072 & 0.010 & 0.352 & 497.913 & 31.003 & 574.587 \\
\hline 0.721 & 0.048 & 0.009 & 0.243 & 468.607 & 25.481 & 393.948 \\
\hline 0.654 & 0.053 & 0.007 & 0.187 & 479.388 & 24.218 & 449.572 \\
\hline 0.531 & 0.055 & 0.009 & 0.076 & 561.449 & 27.147 & 532.800 \\
\hline
\end{tabular}




\begin{tabular}{|c|c|c|c|c|c|c|}
\hline 0.696 & 0.059 & 0.009 & 0.353 & 487.761 & 26.684 & 508.479 \\
\hline 0.686 & 0.060 & 0.009 & 0.325 & 491.346 & 26.677 & 504.162 \\
\hline 0.737 & 0.059 & 0.008 & 0.010 & 435.553 & 22.432 & 457.368 \\
\hline 0.672 & 0.056 & 0.009 & 0.179 & 473.401 & 24.229 & 442.373 \\
\hline 0.627 & 0.061 & 0.008 & 0.306 & 512.814 & 26.632 & 527.982 \\
\hline 0.669 & 0.053 & 0.008 & 0.130 & 518.765 & 29.095 & 463.175 \\
\hline 1.021 & 0.056 & 0.009 & 0.223 & 386.563 & 24.395 & 406.953 \\
\hline 1.023 & 0.044 & 0.008 & 0.171 & 435.553 & 31.155 & 385.648 \\
\hline 0.789 & 0.054 & 0.009 & -0.183 & 469.207 & 27.966 & 470.868 \\
\hline 0.928 & 0.043 & 0.007 & 0.160 & 456.607 & 31.104 & 385.648 \\
\hline 0.262 & 0.058 & 0.002 & 0.300 & 499.703 & 10.540 & 505.397 \\
\hline 0.204 & 0.057 & 0.002 & 0.134 & 512.814 & 8.671 & 510.323 \\
\hline 0.221 & 0.060 & 0.002 & 0.218 & 493.735 & 8.684 & 513.390 \\
\hline 0.274 & 0.056 & 0.002 & 0.081 & 502.089 & 11.158 & 498.585 \\
\hline 0.206 & 0.057 & 0.002 & 0.074 & 492.540 & 8.064 & 489.847 \\
\hline 0.211 & 0.058 & 0.002 & 0.309 & 521.738 & 9.284 & 521.320 \\
\hline 0.283 & 0.057 & 0.002 & 0.354 & 494.929 & 11.164 & 499.827 \\
\hline 0.241 & 0.057 & 0.002 & 0.128 & 504.474 & 9.916 & 496.719 \\
\hline 0.259 & 0.060 & 0.002 & 0.163 & 516.385 & 11.145 & 520.104 \\
\hline 0.250 & 0.059 & 0.002 & 0.029 & 496.123 & 9.922 & 506.014 \\
\hline 0.252 & 0.056 & 0.002 & 0.301 & 478.790 & 9.315 & 484.191 \\
\hline 0.252 & 0.058 & 0.002 & 0.041 & 494.332 & 9.924 & 496.719 \\
\hline 0.235 & 0.059 & 0.002 & 0.314 & 526.492 & 10.517 & 520.712 \\
\hline 0.306 & 0.059 & 0.002 & 0.283 & 513.409 & 13.006 & 519.496 \\
\hline 0.259 & 0.057 & 0.002 & 0.442 & 472.802 & 9.319 & 474.692 \\
\hline 0.227 & 0.059 & 0.002 & 0.331 & 519.954 & 9.904 & 525.565 \\
\hline 0.234 & 0.055 & 0.002 & 0.221 & 512.219 & 9.910 & 494.849 \\
\hline 0.238 & 0.058 & 0.002 & 0.197 & 508.050 & 9.913 & 513.390 \\
\hline 0.285 & 0.057 & 0.002 & -0.009 & 505.666 & 11.774 & 501.687 \\
\hline 0.265 & 0.058 & 0.002 & 0.121 & 496.720 & 10.542 & 497.341 \\
\hline 0.283 & 0.057 & 0.002 & 0.455 & 494.929 & 11.164 & 501.067 \\
\hline 0.244 & 0.057 & 0.002 & 0.422 & 516.385 & 10.526 & 510.323 \\
\hline 0.345 & 0.058 & 0.002 & 0.170 & 449.396 & 11.204 & 459.953 \\
\hline 0.202 & 0.057 & 0.001 & 0.005 & 549.028 & 9.881 & 538.791 \\
\hline 0.282 & 0.059 & 0.002 & 0.398 & 482.380 & 10.554 & 496.096 \\
\hline 0.387 & 0.060 & 0.002 & 0.373 & 436.758 & 11.838 & 463.175 \\
\hline 0.370 & 0.059 & 0.002 & 0.165 & 457.208 & 12.441 & 461.243 \\
\hline 0.246 & 0.059 & 0.002 & 0.119 & 515.195 & 10.527 & 526.774 \\
\hline 0.217 & 0.058 & 0.002 & 0.362 & 531.243 & 9.895 & 529.792 \\
\hline 0.110 & 0.837 & 0.030 & 0.318 & 2509.819 & 131.818 & 4089.085 \\
\hline 0.122 & 0.817 & 0.020 & 0.026 & 3514.759 & 310.268 & 4512.187 \\
\hline 1.111 & 0.720 & 0.046 & 0.010 & 917.758 & 155.959 & 2852.620 \\
\hline 0.622 & 0.749 & 0.035 & -0.040 & 1110.532 & 129.956 & 3076.746 \\
\hline 0.260 & 0.715 & 0.026 & 0.340 & 1115.956 & 54.912 & 3020.266 \\
\hline 0.175 & 0.716 & 0.027 & 0.468 & 1256.462 & 47.292 & 3128.387 \\
\hline 1.427 & 0.622 & 0.041 & 0.001 & 359.794 & 29.460 & 1864.016 \\
\hline 0.351 & 0.814 & 0.040 & 0.165 & 982.290 & 56.694 & 2995.064 \\
\hline 0.780 & 0.776 & 0.040 & 0.432 & 753.546 & 72.924 & 2694.057 \\
\hline
\end{tabular}




\begin{tabular}{|c|c|c|c|c|c|c|}
\hline 0.118 & 0.809 & 0.017 & 0.136 & 2019.983 & 87.825 & 3804.791 \\
\hline 0.152 & 0.808 & 0.028 & -0.119 & 2404.138 & 164.886 & 3988.319 \\
\hline 0.349 & 0.596 & 0.016 & 0.258 & 870.622 & 43.953 & 2584.689 \\
\hline 0.193 & 0.613 & 0.021 & 0.567 & 978.968 & 31.040 & 2738.819 \\
\hline 0.110 & 0.802 & 0.022 & 0.474 & 1575.226 & 47.803 & 3507.883 \\
\hline 0.107 & 0.824 & 0.022 & 0.693 & 2452.790 & 121.845 & 4033.281 \\
\hline 0.340 & 0.751 & 0.034 & 0.140 & 1266.004 & 93.346 & 3196.886 \\
\hline 0.070 & 0.852 & 0.023 & 0.406 & 4171.496 & 265.876 & 4769.089 \\
\hline 0.045 & 0.830 & 0.017 & 0.462 & 5094.433 & 275.032 & 5016.208 \\
\hline 0.016 & 0.842 & 0.017 & 0.481 & 7852.838 & 303.428 & 5699.475 \\
\hline 0.015 & 0.855 & 0.018 & 0.179 & 9032.606 & 413.256 & 5979.467 \\
\hline 0.023 & 0.840 & 0.035 & 0.064 & 13323.853 & 2124.093 & 6775.954 \\
\hline 0.021 & 0.843 & 0.029 & -0.153 & 17414.093 & 5011.250 & 7504.492 \\
\hline 0.039 & 0.820 & 0.030 & 0.195 & 7716.024 & 701.457 & 5695.763 \\
\hline 0.028 & 0.822 & 0.026 & 0.165 & 15964.792 & 4833.377 & 7199.990 \\
\hline 0.033 & 0.842 & 0.024 & 0.169 & 12066.412 & 2193.893 & 6578.169 \\
\hline 0.017 & 0.861 & 0.029 & 0.244 & 15457.826 & 2627.830 & 7174.304 \\
\hline 0.043 & 0.792 & 0.027 & 0.142 & 7637.647 & 740.212 & 5590.020 \\
\hline 0.026 & 0.830 & 0.026 & 0.288 & 8434.055 & 593.508 & 5834.597 \\
\hline 0.037 & 0.821 & 0.025 & 0.358 & 7230.798 & 558.902 & 5552.176 \\
\hline 0.031 & 0.835 & 0.022 & 0.236 & 7498.152 & 511.238 & 5630.479 \\
\hline 0.044 & 0.844 & 0.024 & 0.200 & 6728.793 & 548.543 & 5453.255 \\
\hline 0.026 & 0.825 & 0.025 & 0.337 & 8185.402 & 543.562 & 5750.074 \\
\hline 0.208 & 0.836 & 0.032 & -0.348 & 3644.247 & 575.407 & 4486.816 \\
\hline 0.037 & 0.841 & 0.025 & 0.298 & 13404.941 & 3446.985 & 6788.710 \\
\hline 0.024 & 0.843 & 0.026 & 0.232 & 10165.445 & 926.538 & 6175.782 \\
\hline 0.040 & 0.839 & 0.035 & 0.334 & 10426.470 & 1677.526 & 6182.713 \\
\hline 0.047 & 0.855 & 0.020 & 0.126 & 6885.757 & 612.868 & 5508.402 \\
\hline 0.031 & 0.845 & 0.026 & 0.175 & 11174.368 & 1630.595 & 6427.091 \\
\hline 0.022 & 0.823 & 0.032 & 0.534 & 16778.016 & 4563.620 & 7363.613 \\
\hline 0.048 & 0.818 & 0.031 & 0.171 & 6402.912 & 527.299 & 5343.646 \\
\hline 0.037 & 0.868 & 0.032 & 0.230 & 6796.532 & 472.486 & 5503.919 \\
\hline 0.016 & 0.846 & 0.026 & 0.131 & 14092.192 & 1783.822 & 6930.456 \\
\hline 0.040 & 0.868 & 0.041 & 0.185 & 8416.609 & 907.367 & 5847.491 \\
\hline 0.085 & 0.808 & 0.051 & 0.385 & 3367.342 & 196.346 & 4441.792 \\
\hline 0.060 & 0.838 & 0.034 & 0.116 & 7334.943 & 934.167 & 5560.708 \\
\hline 0.129 & 0.824 & 0.034 & -0.015 & 3121.846 & 250.549 & 4319.636 \\
\hline 0.165 & 0.814 & 0.032 & 0.216 & 3251.604 & 351.927 & 4385.257 \\
\hline 0.058 & 0.829 & 0.026 & 0.177 & 6034.446 & 545.047 & 5238.417 \\
\hline 0.224 & 0.842 & 0.061 & 0.340 & 3753.203 & 665.125 & 4569.030 \\
\hline 0.071 & 0.813 & 0.022 & -0.024 & 5369.277 & 495.626 & 5080.922 \\
\hline 0.082 & 0.837 & 0.027 & 0.388 & 4964.437 & 470.766 & 4957.914 \\
\hline 0.025 & 0.833 & 0.033 & 0.546 & 9447.655 & 794.397 & 6044.822 \\
\hline 0.016 & 0.812 & 0.025 & 0.395 & 11231.065 & 858.425 & 6375.104 \\
\hline 0.016 & 0.847 & 0.025 & 0.314 & 14305.905 & 1919.085 & 6984.136 \\
\hline 0.007 & 0.833 & 0.025 & 0.325 & 21751.289 & 4088.008 & 8204.850 \\
\hline 0.024 & 0.857 & 0.033 & 0.116 & 14512.759 & 2902.552 & 6984.136 \\
\hline 0.048 & 0.812 & 0.041 & 0.195 & 6282.415 & 494.978 & 5295.157 \\
\hline 0.064 & 0.857 & 0.041 & -0.145 & 5562.546 & 487.230 & 5134.026 \\
\hline
\end{tabular}




\begin{tabular}{|c|c|c|c|c|c|c|}
\hline 0.168 & 0.812 & 0.039 & 0.132 & 2562.017 & 210.001 & 4099.888 \\
\hline 0.052 & 0.847 & 0.033 & 0.079 & 7907.896 & 984.385 & 5721.470 \\
\hline 0.006 & 0.833 & 0.018 & 0.238 & 20405.963 & 2966.506 & 8026.677 \\
\hline 0.029 & 0.816 & 0.035 & 0.426 & 9594.840 & 951.092 & 6083.632 \\
\hline 0.081 & 0.841 & 0.036 & 0.376 & 5535.288 & 610.510 & 5165.859 \\
\hline 0.167 & 0.805 & 0.063 & -0.001 & 5082.723 & 1016.545 & 5101.163 \\
\hline 0.067 & 0.834 & 0.028 & 0.129 & 4400.269 & 287.658 & 4792.895 \\
\hline 0.032 & 0.847 & 0.036 & -0.076 & 7676.954 & 569.905 & 5699.475 \\
\hline 0.015 & 0.850 & 0.032 & -0.105 & 19407.709 & 5731.810 & 7944.810 \\
\hline 0.101 & 0.856 & 0.036 & 0.052 & 7417.064 & 1613.898 & 5657.871 \\
\hline 0.055 & 0.811 & 0.028 & 0.041 & 6885.757 & 721.022 & 5443.765 \\
\hline 0.009 & 0.831 & 0.017 & -0.269 & 17542.597 & 2347.249 & 7504.492 \\
\hline 0.072 & 0.864 & 0.041 & 0.462 & 4795.103 & 382.218 & 4950.193 \\
\hline 0.038 & 0.848 & 0.027 & 0.228 & 8451.454 & 873.213 & 5860.224 \\
\hline 0.125 & 0.800 & 0.034 & 0.299 & 3029.838 & 227.238 & 4279.927 \\
\hline 0.042 & 0.846 & 0.025 & 0.209 & 11656.978 & 2514.250 & 6479.988 \\
\hline 0.041 & 0.856 & 0.016 & 0.055 & 8293.144 & 886.290 & 5847.491 \\
\hline 0.017 & 0.833 & 0.015 & 0.281 & 9851.590 & 600.374 & 6123.456 \\
\hline 0.031 & 0.844 & 0.017 & 0.470 & 5662.409 & 245.492 & 5190.626 \\
\hline 0.006 & 0.836 & 0.019 & -0.029 & 19984.479 & 2545.193 & 7894.883 \\
\hline 0.039 & 0.858 & 0.015 & 0.043 & 8203.485 & 829.925 & 5808.308 \\
\hline 0.021 & 0.839 & 0.017 & 0.303 & 8571.952 & 493.350 & 5869.669 \\
\hline 0.005 & 0.858 & 0.011 & 0.087 & 15893.982 & 885.459 & 7263.882 \\
\hline 0.076 & 0.834 & 0.028 & 0.118 & 4934.523 & 429.089 & 4995.665 \\
\hline 1.304 & 0.759 & 0.016 & -0.129 & 316.363 & 20.755 & 1859.146 \\
\hline 0.039 & 0.855 & 0.019 & 0.373 & 6330.885 & 417.004 & 5369.617 \\
\hline 0.059 & 0.824 & 0.018 & -0.069 & 7735.470 & 1066.961 & 5657.871 \\
\hline 0.277 & 0.830 & 0.047 & 0.413 & 2224.059 & 253.715 & 3902.873 \\
\hline 0.042 & 0.844 & 0.020 & 0.380 & 5524.353 & 317.773 & 5146.241 \\
\hline 0.005 & 0.852 & 0.017 & 0.247 & 16559.472 & 1016.930 & 7360.008 \\
\hline 0.025 & 0.860 & 0.018 & 0.210 & 8416.609 & 563.193 & 5841.065 \\
\hline 0.031 & 0.823 & 0.020 & 0.132 & 5366.474 & 218.955 & 5074.085 \\
\hline 0.007 & 0.852 & 0.012 & 0.079 & 11699.112 & 432.457 & 6500.398 \\
\hline 0.003 & 0.837 & 0.015 & 0.221 & 25371.407 & 3234.602 & 8790.302 \\
\hline 0.022 & 0.841 & 0.018 & 0.034 & 10098.504 & 852.644 & 6180.408 \\
\hline 0.080 & 0.817 & 0.021 & -0.179 & 7146.254 & 1161.706 & 5543.571 \\
\hline 0.018 & 0.857 & 0.017 & -0.028 & 16730.087 & 3642.841 & 7405.897 \\
\hline 0.018 & 0.849 & 0.013 & 0.106 & 10323.325 & 729.932 & 6229.949 \\
\hline 0.021 & 0.813 & 0.016 & 0.283 & 8363.985 & 471.653 & 5791.524 \\
\hline 0.149 & 0.846 & 0.032 & 0.158 & 6209.021 & 1494.764 & 5306.135 \\
\hline 0.099 & 0.806 & 0.027 & 0.279 & 4344.653 & 415.497 & 4758.847 \\
\hline 0.032 & 0.829 & 0.025 & 0.345 & 8968.779 & 861.240 & 5918.613 \\
\hline 0.040 & 0.848 & 0.016 & -0.092 & 13323.853 & 3668.887 & 6930.456 \\
\hline 0.015 & 0.856 & 0.018 & 0.223 & 10085.032 & 560.280 & 6207.729 \\
\hline 0.107 & 0.847 & 0.021 & -0.024 & 5369.277 & 743.438 & 5114.436 \\
\hline 0.019 & 0.837 & 0.019 & 0.294 & 8756.868 & 484.809 & 5879.027 \\
\hline 0.024 & 0.837 & 0.016 & 0.119 & 8756.868 & 606.012 & 5912.622 \\
\hline 0.007 & 0.848 & 0.014 & 0.025 & 17012.457 & 1439.516 & 7439.837 \\
\hline 0.005 & 0.855 & 0.015 & -0.028 & 17344.496 & 1211.842 & 7504.492 \\
\hline
\end{tabular}




\begin{tabular}{|c|c|c|c|c|c|c|}
\hline 0.011 & 0.847 & 0.014 & 0.061 & 12562.535 & 813.852 & 6653.305 \\
\hline 0.010 & 0.848 & 0.014 & 0.064 & 17710.046 & 2547.335 & 7582.822 \\
\hline 0.005 & 0.854 & 0.021 & 0.026 & 21270.161 & 3015.310 & 8149.969 \\
\hline 0.129 & 0.862 & 0.048 & 0.409 & 3352.029 & 294.900 & 4484.366 \\
\hline 0.386 & 0.803 & 0.026 & 0.259 & 1696.266 & 197.240 & 3559.484 \\
\hline 0.013 & 0.854 & 0.013 & -0.121 & 22136.904 & 8854.762 & 8314.938 \\
\hline 0.011 & 0.838 & 0.017 & 0.144 & 11442.078 & 630.482 & 6436.101 \\
\hline 0.021 & 0.866 & 0.026 & 0.386 & 10426.470 & 877.475 & 6302.052 \\
\hline 0.017 & 0.843 & 0.018 & -0.001 & 12016.633 & 1124.492 & 6546.485 \\
\hline 0.068 & 0.828 & 0.017 & -0.217 & 6973.764 & 929.835 & 5490.351 \\
\hline 0.177 & 0.829 & 0.019 & 0.087 & 4403.525 & 763.877 & 4763.515 \\
\hline 0.024 & 0.847 & 0.014 & 0.008 & 10823.297 & 1117.083 & 6322.320 \\
\hline 0.005 & 0.848 & 0.019 & 0.208 & 19896.771 & 2284.797 & 7924.305 \\
\hline 0.115 & 0.820 & 0.024 & 0.030 & 5425.091 & 821.983 & 5094.461 \\
\hline 0.033 & 0.860 & 0.017 & 0.190 & 7657.331 & 570.941 & 5706.860 \\
\hline 0.111 & 0.835 & 0.038 & 0.382 & 3785.534 & 336.387 & 4602.325 \\
\hline 0.025 & 0.839 & 0.019 & 0.172 & 14512.759 & 3073.290 & 6952.270 \\
\hline 0.168 & 0.848 & 0.031 & -0.229 & 4069.439 & 601.167 & 4800.109 \\
\hline 0.006 & 0.855 & 0.016 & 0.355 & 16830.329 & 1201.213 & 7433.139 \\
\hline 0.031 & 0.851 & 0.013 & 0.180 & 6462.327 & 340.911 & 5384.886 \\
\hline 0.024 & 0.857 & 0.016 & 0.453 & 5860.207 & 209.576 & 5234.910 \\
\hline 0.011 & 0.859 & 0.017 & 0.071 & 14613.750 & 1334.666 & 7034.125 \\
\hline 0.060 & 0.828 & 0.023 & 0.318 & 5278.958 & 399.669 & 5053.291 \\
\hline 0.162 & 0.821 & 0.026 & 0.127 & 2173.642 & 140.934 & 3920.120 \\
\hline 0.069 & 0.842 & 0.019 & 0.204 & 3173.275 & 139.704 & 4374.383 \\
\hline 0.791 & 0.790 & 0.049 & 0.420 & 928.931 & 113.869 & 2917.769 \\
\hline 0.120 & 0.837 & 0.023 & 0.204 & 2911.925 & 198.888 & 4270.888 \\
\hline 0.025 & 0.874 & 0.020 & 0.429 & 7945.594 & 490.469 & 5767.551 \\
\hline 0.053 & 0.847 & 0.022 & 0.064 & 6751.452 & 656.898 & 5467.325 \\
\hline 0.007 & 0.853 & 0.013 & 0.197 & 19279.418 & 2652.195 & 7824.250 \\
\hline 0.097 & 0.830 & 0.027 & 0.206 & 3640.583 & 268.607 & 4534.607 \\
\hline 0.183 & 0.827 & 0.021 & 0.167 & 4690.080 & 920.483 & 4902.588 \\
\hline 0.039 & 0.844 & 0.044 & 0.334 & 7396.631 & 619.253 & 5598.242 \\
\hline 0.028 & 0.828 & 0.035 & 0.191 & 12814.661 & 2237.481 & 6695.856 \\
\hline 0.060 & 0.842 & 0.024 & 0.048 & 5507.915 & 448.793 & 5121.008 \\
\hline 0.088 & 0.810 & 0.030 & 0.227 & 2887.258 & 143.085 & 4224.445 \\
\hline 0.012 & 0.864 & 0.017 & 0.000 & 13611.117 & 1199.878 & 6864.411 \\
\hline 0.045 & 0.851 & 0.026 & -0.226 & 8520.586 & 1053.454 & 5853.877 \\
\hline 0.010 & 0.840 & 0.017 & 0.217 & 14471.916 & 1165.984 & 7022.108 \\
\hline 0.062 & 0.846 & 0.025 & -0.113 & 6426.744 & 676.499 & 5399.929 \\
\hline 0.050 & 0.839 & 0.019 & 0.103 & 9251.149 & 1474.402 & 5982.276 \\
\hline 0.181 & 0.819 & 0.029 & 0.043 & 3717.089 & 524.205 & 4546.212 \\
\hline 0.064 & 0.833 & 0.020 & -0.126 & 5855.011 & 553.852 & 5238.417 \\
\hline 0.083 & 0.826 & 0.024 & -0.150 & 4436.001 & 362.946 & 4835.431 \\
\hline 0.043 & 0.858 & 0.014 & -0.321 & 10859.277 & 2053.121 & 6390.231 \\
\hline 0.008 & 0.838 & 0.018 & -0.088 & 15046.471 & 1130.100 & 7076.053 \\
\hline 0.310 & 0.822 & 0.022 & -0.066 & 2841.787 & 487.310 & 4238.602 \\
\hline 2.653 & 0.766 & 0.042 & 0.139 & 436.156 & 81.000 & 2160.970 \\
\hline 0.053 & 0.840 & 0.024 & 0.212 & 3916.772 & 173.350 & 4640.888 \\
\hline 0.025 & 0.834 & 0.016 & 0.070 & 10652.683 & 1110.706 & 6270.871 \\
\hline
\end{tabular}




$\begin{array}{lllllll}0.189 & 0.834 & 0.025 & 0.047 & 8936.628 & 5064.089 & 5979.467 \\ 0.056 & 0.859 & 0.018 & 0.288 & 5802.813 & 476.944 & 5226.679 \\ 0.019 & 0.831 & 0.016 & 0.159 & 8605.970 & 461.034 & 5857.055 \\ 0.120 & 0.855 & 0.026 & -0.126 & 5256.179 & 792.598 & 5101.163 \\ 0.170 & 0.836 & 0.017 & -0.043 & 3930.801 & 561.543 & 4686.115 \\ 0.040 & 0.849 & 0.020 & 0.444 & 6282.415 & 418.828 & 5354.114 \\ 0.026 & 0.841 & 0.018 & 0.348 & 7754.858 & 465.957 & 5699.475 \\ 0.028 & 0.855 & 0.014 & -0.145 & 6525.885 & 320.335 & 5404.894 \\ 0.032 & 0.845 & 0.015 & 0.399 & 5976.038 & 289.605 & 5272.276 \\ 0.028 & 0.838 & 0.018 & -0.063 & 7538.317 & 475.389 & 5626.505\end{array}$




\begin{tabular}{|c|c|c|c|c|}
\hline Error $2 \sigma$ & alAge ${ }^{207} \mathrm{~Pb} /{ }^{20 \epsilon}$ & Error $2 \sigma$ & alAge $207 \mathrm{~Pb} \mathrm{c}$ & Error $2 \sigma$ \\
\hline 101.020 & 1835.353 & 86.697 & 1943.094 & 78.102 \\
\hline 89.667 & 1966.596 & 105.906 & 1935.338 & 67.059 \\
\hline 102.952 & 1927.673 & 109.360 & 1859.215 & 77.441 \\
\hline 99.520 & 1910.892 & 94.728 & 1943.515 & 67.073 \\
\hline 92.378 & 2000.193 & 95.944 & 1957.502 & 88.297 \\
\hline 119.948 & 1923.116 & 111.012 & 2002.218 & 62.645 \\
\hline 108.533 & 1998.748 & 107.337 & 1843.730 & 61.018 \\
\hline 103.980 & 1832.121 & 109.600 & 1982.234 & 68.169 \\
\hline 101.612 & 1979.834 & 91.177 & 1988.019 & 77.816 \\
\hline 100.413 & 1929.190 & 96.296 & 1902.590 & 66.723 \\
\hline 100.014 & 1843.404 & 99.776 & 2013.927 & 79.029 \\
\hline 191.061 & 2379.728 & 186.645 & 1974.421 & 79.069 \\
\hline 101.842 & 1846.612 & 98.137 & 1980.208 & 78.631 \\
\hline 111.961 & 1890.811 & 106.225 & 1701.423 & 55.434 \\
\hline 113.405 & 1901.658 & 120.896 & 1694.696 & 61.011 \\
\hline 83.184 & 1870.454 & 88.291 & 1911.893 & 53.947 \\
\hline 107.986 & 1881.450 & 101.347 & 1936.721 & 67.328 \\
\hline 104.836 & 1906.282 & 102.910 & 1977.164 & 78.400 \\
\hline 94.966 & 1957.705 & 92.914 & 1958.539 & 61.563 \\
\hline 98.531 & 1930.704 & 110.979 & 1820.782 & 61.265 \\
\hline 110.444 & 1819.120 & 98.154 & 1906.697 & 72.701 \\
\hline 95.012 & 1836.967 & 101.418 & 1920.835 & 62.019 \\
\hline 90.116 & 1917.017 & 97.974 & 1909.782 & 66.873 \\
\hline 115.541 & 1843.404 & 89.962 & 2030.610 & 78.972 \\
\hline 89.049 & 1930.704 & 89.762 & 1891.464 & 55.783 \\
\hline 138.735 & 2087.113 & 103.386 & 1981.528 & 88.125 \\
\hline 88.787 & 1784.441 & 83.416 & 2055.776 & 73.874 \\
\hline 101.651 & 1878.317 & 88.276 & 1975.708 & 83.652 \\
\hline 85.417 & 1926.156 & 88.146 & 1837.682 & 66.131 \\
\hline 86.908 & 328.797 & 58.315 & 474.807 & 24.993 \\
\hline 84.130 & 741.630 & 115.880 & 514.845 & 30.643 \\
\hline 75.766 & 408.153 & 68.397 & 484.679 & 29.689 \\
\hline 76.789 & 495.398 & 74.613 & 505.828 & 31.924 \\
\hline 76.725 & 670.656 & 98.594 & 507.727 & 25.305 \\
\hline 83.080 & 856.315 & 120.340 & 516.186 & 24.046 \\
\hline 71.060 & 751.514 & 102.851 & 500.248 & 27.548 \\
\hline 79.008 & 475.982 & 79.050 & 510.979 & 32.022 \\
\hline 61.342 & 345.831 & 51.810 & 495.907 & 28.410 \\
\hline 67.411 & 635.704 & 76.201 & 527.615 & 28.659 \\
\hline 77.739 & 674.109 & 119.600 & 474.507 & 29.067 \\
\hline 72.503 & 529.762 & 76.724 & 452.525 & 21.169 \\
\hline 85.490 & 674.109 & 108.727 & 499.234 & 28.947 \\
\hline 74.610 & 424.372 & 60.625 & 517.840 & 22.964 \\
\hline 61.401 & 632.166 & 88.378 & 490.303 & 28.817 \\
\hline 72.484 & 977.437 & 130.870 & 489.197 & 30.331 \\
\hline 65.658 & 94.333 & 17.134 & 473.416 & 25.657 \\
\hline 61.342 & 320.212 & 44.272 & 481.633 & 24.165 \\
\hline 84.939 & 424.372 & 70.601 & 563.829 & 27.287 \\
\hline
\end{tabular}




$\begin{array}{lcccc}86.050 & 555.987 & 89.034 & 486.705 & 26.613 \\ 72.135 & 585.440 & 84.618 & 489.864 & 26.494 \\ 61.090 & 559.699 & 72.342 & 433.854 & 22.256 \\ 72.919 & 464.219 & 77.507 & 473.534 & 24.308 \\ 65.030 & 646.271 & 81.312 & 510.567 & 26.332 \\ 66.511 & 333.072 & 47.044 & 521.635 & 28.946 \\ 66.037 & 448.399 & 73.797 & 385.845 & 24.292 \\ 62.605 & -88.460 & -14.942 & 441.441 & 31.179 \\ 79.808 & 383.513 & 64.272 & 470.410 & 27.910 \\ 61.770 & -150.333 & -23.956 & 463.663 & 31.142 \\ & & & & \\ 17.238 & 533.535 & 17.448 & 499.169 & 10.352 \\ 17.193 & 499.252 & 14.838 & 513.032 & 8.537 \\ 17.165 & 599.964 & 19.031 & 492.045 & 8.535 \\ 18.087 & 448.399 & 12.834 & 502.918 & 10.963 \\ 15.011 & 479.884 & 13.542 & 492.734 & 7.943 \\ 12.431 & 522.189 & 14.455 & 521.731 & 9.120 \\ 14.932 & 506.935 & 13.247 & 494.743 & 10.944 \\ 18.105 & 475.982 & 16.819 & 504.920 & 9.773 \\ 20.991 & 599.964 & 22.035 & 514.988 & 10.941 \\ 22.716 & 552.267 & 20.734 & 495.238 & 9.770 \\ 16.642 & 460.278 & 16.380 & 479.062 & 9.188 \\ 17.318 & 533.535 & 17.448 & 493.720 & 9.754 \\ 15.544 & 548.538 & 15.003 & 526.122 & 10.311 \\ 15.554 & 548.538 & 15.940 & 512.835 & 12.732 \\ 14.336 & 495.398 & 15.617 & 472.470 & 9.168 \\ 17.829 & 578.128 & 17.549 & 518.981 & 9.719 \\ 17.335 & 424.372 & 13.813 & 513.596 & 9.768 \\ 18.725 & 525.980 & 16.352 & 507.762 & 9.741 \\ 18.058 & 506.935 & 16.780 & 505.646 & 11.561 \\ 18.099 & 518.389 & 17.968 & 496.382 & 10.366 \\ 18.064 & 479.884 & 18.620 & 495.160 & 10.995 \\ 17.193 & 487.660 & 17.998 & 516.849 & 10.371 \\ 17.660 & 537.299 & 19.387 & 448.161 & 11.000 \\ 14.624 & 503.098 & 10.536 & 549.826 & 9.685 \\ 14.174 & 567.096 & 17.301 & 481.080 & 10.346 \\ 16.027 & 585.440 & 19.679 & 434.701 & 11.592 \\ 17.648 & 548.538 & 15.003 & 455.895 & 12.176 \\ 15.493 & 570.782 & 15.453 & 514.277 & 10.311 \\ 14.695 & 518.389 & 14.375 & 531.460 & 9.716 \\ 222.636 & 4986.038 & 178.711 & -6.510 & 121.171 \\ 343.377 & 4951.665 & 121.216 & 176.223 & 124.623 \\ 493.723 & 4771.159 & 304.824 & 153.718 & 62.167 \\ 421.686 & 4827.717 & 225.594 & 144.546 & 56.037 \\ 160.929 & 4761.163 & 173.133 & 200.933 & 41.233 \\ 118.933 & 4763.168 & 179.617 & 229.490 & 47.770 \\ 198.074 & 4560.250 & 300.595 & 101.792 & 20.785 \\ 198.568 & 4946.432 & 243.068 & 31.646 & 54.559 \\ 265.324 & 4878.312 & 251.459 & 63.973 & 41.217\end{array}$




\begin{tabular}{|c|c|c|c|c|}
\hline 156.235 & 4937.664 & 103.758 & 97.770 & 55.676 \\
\hline 296.321 & 4935.904 & 171.046 & 131.288 & 105.298 \\
\hline 131.984 & 4498.277 & 120.759 & 294.026 & 24.003 \\
\hline 87.072 & 4539.118 & 155.500 & 312.456 & 29.273 \\
\hline 103.005 & 4925.292 & 135.108 & 87.761 & 52.108 \\
\hline 147.087 & 4963.796 & 132.529 & 57.886 & 87.873 \\
\hline 243.709 & 4831.531 & 218.738 & 165.305 & 61.010 \\
\hline 329.357 & 5011.250 & 135.280 & -214.530 & 189.955 \\
\hline 245.751 & 4974.107 & 101.879 & 192.279 & 179.259 \\
\hline 208.772 & 4994.495 & 100.839 & 949.020 & 317.425 \\
\hline 298.973 & 5016.235 & 105.605 & -484.607 & 526.826 \\
\hline 1115.030 & 4991.118 & 207.963 & -6698.243 & 6170.266 \\
\hline 2177.229 & 4996.180 & 171.873 & 0.000 & 4155.742 \\
\hline 544.448 & 4956.877 & 181.349 & 0.000 & 569.772 \\
\hline 2039.997 & 4960.341 & 156.896 & 0.000 & 3281.484 \\
\hline 1214.431 & 4994.495 & 142.361 & -4742.728 & 2748.354 \\
\hline 1226.377 & 5026.151 & 169.290 & -2406.996 & 3450.406 \\
\hline 547.594 & 4907.418 & 167.298 & 1609.095 & 472.860 \\
\hline 430.115 & 4974.107 & 155.815 & 0.000 & 572.979 \\
\hline 399.945 & 4958.610 & 150.993 & 2111.130 & 389.962 \\
\hline 397.446 & 4982.640 & 131.279 & 1294.628 & 357.764 \\
\hline 458.685 & 4997.862 & 142.119 & -30.103 & 380.375 \\
\hline 340.597 & 4965.520 & 150.470 & 0.000 & 523.338 \\
\hline 711.324 & 4984.340 & 190.788 & 2.541 & 204.962 \\
\hline 1782.036 & 4992.807 & 148.419 & -11193.841 & 11797.549 \\
\hline 565.289 & 4996.180 & 154.093 & -728.047 & 954.297 \\
\hline 913.355 & 4989.427 & 208.141 & -353.954 & 1248.184 \\
\hline 487.469 & 5016.235 & 117.339 & -890.326 & 402.674 \\
\hline 998.495 & 4999.543 & 153.832 & -1334.809 & 1283.412 \\
\hline 1984.520 & 4962.070 & 192.936 & 0.000 & 4007.151 \\
\hline 417.472 & 4953.404 & 187.721 & 1189.794 & 379.418 \\
\hline 366.928 & 5037.628 & 185.719 & -2082.530 & 754.107 \\
\hline 828.642 & 5001.222 & 153.702 & 0.000 & 2939.200 \\
\hline 592.151 & 5037.628 & 237.952 & -6614.516 & 3134.434 \\
\hline 317.271 & 4935.904 & 311.548 & 235.227 & 277.108 \\
\hline 654.201 & 4987.733 & 202.366 & 809.326 & 536.915 \\
\hline 404.577 & 4963.796 & 204.817 & 88.365 & 175.106 \\
\hline 491.196 & 4946.432 & 194.454 & 173.780 & 172.160 \\
\hline 454.198 & 4972.394 & 155.950 & 486.031 & 318.436 \\
\hline 821.399 & 4994.495 & 361.834 & -61.574 & 401.548 \\
\hline 514.958 & 4944.683 & 133.804 & 673.073 & 228.725 \\
\hline 416.313 & 4986.038 & 160.840 & 22.991 & 265.778 \\
\hline 440.768 & 4979.233 & 197.257 & 192.377 & 902.121 \\
\hline 467.348 & 4942.932 & 152.184 & \#NUM! & \#VALUE! \\
\hline 936.018 & 5002.898 & 147.665 & 0.000 & 3391.160 \\
\hline 1600.327 & 4979.233 & 149.437 & 0.000 & 6066.830 \\
\hline 1368.027 & 5019.549 & 193.285 & -1638.437 & 2947.108 \\
\hline 405.094 & 4942.932 & 249.582 & 1328.142 & 468.519 \\
\hline 526.567 & 5019.549 & 240.142 & -585.610 & 522.277 \\
\hline
\end{tabular}




$\begin{array}{ccccc}397.476 & 4942.932 & 237.407 & 124.561 & 155.375 \\ 635.719 & 5002.898 & 194.918 & 276.740 & 631.691 \\ 1214.368 & 4979.233 & 107.594 & \text { \#VALUE! } & \text { \#VALUE! } \\ 594.641 & 4949.923 & 212.313 & 3187.661 & 1727.165 \\ 577.549 & 4992.807 & 213.723 & -48.540 & 412.158 \\ 1486.432 & 4930.609 & 385.874 & 718.123 & 565.258 \\ 340.502 & 4980.938 & 167.226 & 47.785 & 231.064 \\ 480.176 & 5002.898 & 212.638 & 92.096 & 670.812 \\ 2478.781 & 5007.915 & 188.533 & \text { \#VALUE! } & \text { \#VALUE! } \\ 1209.316 & 5017.893 & 211.033 & -1172.322 & 839.330 \\ 564.919 & 4941.178 & 170.596 & 2176.854 & 428.209 \\ 1019.128 & 4975.818 & 101.792 & \text { \#VALUE! } & \text { \#VALUE! } \\ 418.862 & 5031.082 & 238.743 & -552.576 & 420.327 \\ 586.022 & 5004.573 & 159.344 & 764.786 & 554.463 \\ 340.084 & 4921.737 & 209.174 & 246.466 & 164.433 \\ 1317.964 & 5001.222 & 147.790 & -1776.273 & 1495.844 \\ 647.665 & 5017.893 & 93.792 & -1331.355 & 504.329 \\ 339.372 & 4979.233 & 89.662 & 219.362 & 491.529 \\ 213.917 & 4997.862 & 100.668 & -142.844 & 222.684 \\ 928.810 & 4984.340 & 113.280 & -66.077 & 3618.676 \\ 592.295 & 5021.203 & 87.783 & -1859.514 & 538.210 \\ 327.102 & 4989.427 & 101.097 & 3438.879 & 1001.142 \\ 409.233 & 5021.203 & 64.374 & -2264.366 & 1667.933 \\ 477.527 & 4980.938 & 167.226 & 84.741 & 269.386 \\ 127.728 & 4846.679 & 102.170 & 32.173 & 7.522 \\ 354.340 & 5016.235 & 111.472 & -722.033 & 325.290 \\ 755.822 & 4963.796 & 108.432 & 3106.419 & 756.651 \\ 418.470 & 4974.107 & 281.666 & 24.267 & 159.822 \\ 277.030 & 4997.862 & 118.433 & -143.222 & 245.657 \\ 403.360 & 5011.250 & 99.990 & -1798.165 & 2369.308 \\ 372.042 & 5024.504 & 105.164 & -2622.357 & 714.560 \\ 196.750 & 4962.070 & 120.585 & 424.705 & 212.524 \\ 259.152 & 5011.250 & 70.581 & -3121.563 & 1012.100 \\ 1131.274 & 4986.038 & 89.356 & \text { \#VALUE!} & \text { \#VALUE! } \\ 534.978 & 4992.807 & 106.862 & -511.807 & 662.611 \\ 947.619 & 4951.665 & 127.277 & 2259.699 & 483.485 \\ 1612.168 & 5019.549 & 99.571 & \text { \#VALUE!} & \text { \#VALUE! } \\ 445.962 & 5006.245 & 76.656 & -443.475 & 529.363 \\ 329.284 & 4944.683 & 97.312 & 1097.356 & 349.049 \\ 1233.318 & 5001.222 & 189.172 & -216.036 & 454.700 \\ 420.549 & 4932.376 & 165.228 & 444.503 & 208.108 \\ 523.771 & 4972.394 & 149.952 & 161.842 & 638.826 \\ 3410.594 & 5004.573 & 94.426 & \text { \#VALUE!} & \text { \#VALUE!} \\ 535.109 & 5017.893 & 105.516 & 1890.887 & 520.857 \\ 555.888 & 5016.235 & 88.004 & -2549.439 & 2744.870\end{array}$




\begin{tabular}{|c|c|c|c|c|}
\hline 437.217 & 5002.898 & 82.693 & 0.000 & 1055.176 \\
\hline 1083.260 & 5004.573 & 82.623 & 0.000 & 2818.139 \\
\hline 1198.525 & 5014.575 & 123.309 & 0.000 & 10412.026 \\
\hline 367.301 & 5027.797 & 279.970 & -228.966 & 284.118 \\
\hline 341.622 & 4927.067 & 159.531 & 93.914 & 66.586 \\
\hline 3233.587 & 5014.575 & 76.334 & \#VALUE! & \#VALUE! \\
\hline 341.740 & 4987.733 & 101.183 & -1021.769 & 884.809 \\
\hline 534.720 & 5034.359 & 151.147 & 0.000 & 1339.931 \\
\hline 613.084 & 4996.180 & 106.680 & \#VALUE! & \#VALUE! \\
\hline 766.671 & 4970.679 & 102.055 & 1222.915 & 293.775 \\
\hline 793.919 & 4972.394 & 113.963 & 124.703 & 163.208 \\
\hline 651.011 & 5002.898 & 82.693 & -1459.899 & 742.932 \\
\hline 905.635 & 5004.573 & 112.131 & 0.000 & 5013.396 \\
\hline 747.188 & 4956.877 & 145.079 & 521.873 & 260.080 \\
\hline 394.292 & 5024.504 & 99.322 & -1946.945 & 522.533 \\
\hline 405.205 & 4982.640 & 226.755 & 15.002 & 252.858 \\
\hline 1405.246 & 4989.427 & 112.991 & \#VALUE! & \#VALUE! \\
\hline 728.588 & 5004.573 & 182.950 & -149.583 & 238.202 \\
\hline 541.487 & 5016.235 & 93.871 & -2301.500 & 2550.459 \\
\hline 296.169 & 5009.583 & 76.527 & -505.171 & 239.257 \\
\hline 176.116 & 5019.549 & 93.714 & -676.530 & 248.669 \\
\hline 669.588 & 5022.854 & 99.405 & -1848.490 & 1724.495 \\
\hline 386.015 & 4970.679 & 138.074 & 275.461 & 240.799 \\
\hline 261.341 & 4958.610 & 157.033 & 60.226 & 88.335 \\
\hline 196.937 & 4994.495 & 112.702 & -46.916 & 108.848 \\
\hline 349.433 & 4903.814 & 304.161 & 62.278 & 62.541 \\
\hline 290.756 & 4986.038 & 137.012 & -7.602 & 114.234 \\
\hline 316.030 & 5047.387 & 115.501 & -7166.158 & 1711.174 \\
\hline 529.096 & 5002.898 & 129.945 & -237.187 & 368.255 \\
\hline 1092.575 & 5012.914 & 76.398 & 0.000 & 4955.393 \\
\hline 348.005 & 4974.107 & 161.808 & 62.705 & 172.930 \\
\hline 1027.962 & 4968.962 & 126.177 & 190.786 & 193.534 \\
\hline 498.629 & 4997.862 & 260.552 & 261.931 & 743.369 \\
\hline 1100.689 & 4970.679 & 210.113 & 0.000 & 1909.128 \\
\hline 399.040 & 4994.495 & 142.361 & -80.861 & 283.222 \\
\hline 234.319 & 4939.422 & 182.942 & 164.704 & 139.205 \\
\hline 629.105 & 5031.082 & 98.991 & \#VALUE! & \#VALUE! \\
\hline 773.154 & 5009.583 & 153.054 & 139.261 & 589.268 \\
\hline 585.757 & 4991.118 & 101.011 & 0.000 & 1570.644 \\
\hline 638.415 & 5001.222 & 147.790 & -204.650 & 379.508 \\
\hline 961.141 & 4989.427 & 112.991 & \#VALUE! & \#VALUE! \\
\hline 627.064 & 4955.142 & 175.457 & 178.818 & 187.171 \\
\hline 484.478 & 4979.233 & 119.549 & 272.266 & 249.315 \\
\hline 458.532 & 4967.242 & 144.327 & 173.975 & 198.828 \\
\hline 1183.376 & 5021.203 & 81.931 & \#NUM! & \#VALUE! \\
\hline 533.036 & 4987.733 & 107.135 & -10115.085 & 7631.405 \\
\hline 728.510 & 4960.341 & 132.759 & 86.631 & 105.524 \\
\hline 379.630 & 4859.795 & 266.464 & 41.066 & 25.376 \\
\hline 223.306 & 4991.118 & 142.603 & -41.827 & 174.735 \\
\hline 666.280 & 4980.938 & 95.558 & \#VALUE! & \#VALUE! \\
\hline
\end{tabular}




$\begin{array}{ccccc}3321.926 & 4980.938 & 149.309 & 39.586 & 643.188 \\ 427.915 & 5022.854 & 105.252 & -743.060 & 278.708 \\ 312.131 & 4975.818 & 95.804 & 105.023 & 407.670 \\ 776.998 & 5016.235 & 152.540 & -445.224 & 312.337 \\ 702.917 & 4984.340 & 101.356 & 6.008 & 129.634 \\ 331.182 & 5006.245 & 117.933 & -372.809 & 311.637 \\ 334.035 & 4992.807 & 106.862 & 954.149 & 327.702 \\ 291.440 & 5016.235 & 82.137 & -780.551 & 271.038 \\ 244.605 & 4999.543 & 88.749 & -176.235 & 220.322 \\ 376.577 & 4987.733 & 107.135 & 1034.133 & 310.733\end{array}$




\begin{tabular}{|c|c|c|c|c|c|c|}
\hline Sample & ${ }^{207} \mathrm{~Pb} /{ }^{235} \mathrm{U}$ & Error $2 \sigma$ & ${ }^{206} \mathrm{~Pb} /{ }^{238} \mathrm{U}$ & Error $2 \sigma$ & rrelation_6_3ع & ${ }^{8} \mathrm{U} /{ }^{206} \mathrm{~Pb}$ \\
\hline WRS_1 & 0.662 & 0.030 & 0.085 & 0.002 & 0.088 & 11.779 \\
\hline WRS_2 & 0.660 & 0.031 & 0.085 & 0.002 & 0.028 & 11.806 \\
\hline WRS_3 & 0.674 & 0.033 & 0.084 & 0.002 & 0.261 & 11.905 \\
\hline WRS_4 & 0.644 & 0.036 & 0.084 & 0.002 & 0.206 & 11.933 \\
\hline WRS_5 & 0.654 & 0.028 & 0.084 & 0.002 & 0.011 & 11.862 \\
\hline WRS_6 & 0.669 & 0.032 & 0.084 & 0.002 & 0.010 & 11.947 \\
\hline WRS_7 & 0.661 & 0.030 & 0.084 & 0.002 & 0.127 & 11.919 \\
\hline WRS_8 & 0.661 & 0.031 & 0.083 & 0.002 & 0.010 & 12.077 \\
\hline WRS_9 & 0.670 & 0.030 & 0.084 & 0.002 & 0.010 & 11.933 \\
\hline WRS_10 & 0.674 & 0.034 & 0.083 & 0.002 & 0.230 & 11.990 \\
\hline WRS_11 & 0.682 & 0.031 & 0.084 & 0.002 & 0.010 & 11.962 \\
\hline WRS_12 & 0.684 & 0.032 & 0.086 & 0.002 & 0.010 & 11.696 \\
\hline WRS_13 & 0.710 & 0.034 & 0.087 & 0.002 & 0.010 & 11.547 \\
\hline WRS_14 & 0.672 & 0.029 & 0.085 & 0.002 & 0.058 & 11.737 \\
\hline WRS_15 & 0.667 & 0.032 & 0.085 & 0.002 & 0.087 & 11.806 \\
\hline WRS_16 & 0.711 & 0.040 & 0.086 & 0.002 & 0.010 & 11.669 \\
\hline WRS_17 & 0.684 & 0.037 & 0.087 & 0.002 & 0.241 & 11.455 \\
\hline WRS_18 & 0.679 & 0.040 & 0.086 & 0.002 & 0.171 & 11.614 \\
\hline WRS_19 & 0.682 & 0.045 & 0.084 & 0.002 & 0.192 & 11.919 \\
\hline WRS_20 & 0.675 & 0.035 & 0.086 & 0.002 & 0.076 & 11.628 \\
\hline ZA16_84_1 & 0.769 & 0.048 & 0.049 & 0.002 & 0.332 & 20.408 \\
\hline ZA16_84_2 & 0.345 & 0.014 & 0.047 & 0.001 & 0.163 & 21.057 \\
\hline ZA16_84_3 & 0.332 & 0.013 & 0.046 & 0.001 & 0.349 & 21.978 \\
\hline ZA16_84_5 & 0.395 & 0.023 & 0.048 & 0.001 & 0.144 & 20.964 \\
\hline ZA16_84_6 & 0.484 & 0.034 & 0.048 & 0.001 & 0.335 & 21.008 \\
\hline ZA16_84_7 & 0.149 & 0.010 & 0.015 & 0.001 & 0.862 & 64.935 \\
\hline ZA16_84_8 & 0.334 & 0.015 & 0.047 & 0.001 & 0.150 & 21.227 \\
\hline ZA16_84_9 & 0.319 & 0.020 & 0.048 & 0.001 & 0.010 & 20.877 \\
\hline ZA16_84_10 & 0.340 & 0.014 & 0.048 & 0.001 & 0.318 & 21.008 \\
\hline ZA16_84_11 & 0.327 & 0.014 & 0.047 & 0.001 & 0.044 & 21.322 \\
\hline ZA16_84_13 & 0.353 & 0.018 & 0.048 & 0.001 & 0.116 & 20.964 \\
\hline ZA16_84_14 & 0.356 & 0.019 & 0.048 & 0.001 & 0.107 & 21.008 \\
\hline ZA16_84_15 & 0.514 & 0.044 & 0.050 & 0.002 & 0.725 & 19.960 \\
\hline ZA16_84_16 & 0.342 & 0.021 & 0.046 & 0.001 & 0.129 & 21.882 \\
\hline ZA16_84_17 & 0.391 & 0.016 & 0.048 & 0.001 & 0.641 & 20.790 \\
\hline ZA16_84_18 & 0.625 & 0.034 & 0.048 & 0.002 & 0.575 & 20.747 \\
\hline ZA16_84_19 & 0.204 & 0.010 & 0.017 & 0.002 & 0.148 & 60.241 \\
\hline ZA16_84_20 & 0.466 & 0.020 & 0.045 & 0.001 & 0.037 & 22.026 \\
\hline ZA16_84_21 & 0.665 & 0.044 & 0.051 & 0.002 & 0.220 & 19.763 \\
\hline ZA16_84_22 & 0.658 & 0.057 & 0.051 & 0.002 & 0.508 & 19.763 \\
\hline ZA16_84_23 & 0.380 & 0.021 & 0.048 & 0.001 & 0.194 & 20.964 \\
\hline ZA16_84_24 & 0.348 & 0.012 & 0.048 & 0.001 & 0.010 & 20.708 \\
\hline ZA16_84_25 & 0.652 & 0.052 & 0.049 & 0.001 & 0.735 & 20.450 \\
\hline ZA16_84_26 & 0.402 & 0.010 & 0.048 & 0.001 & 0.376 & 20.799 \\
\hline ZA16_84_27 & 0.445 & 0.018 & 0.047 & 0.001 & 0.034 & 21.277 \\
\hline ZA16_84_29 & 2.510 & 0.150 & 0.065 & 0.002 & 0.725 & 15.291 \\
\hline ZA16_84_30 & 0.162 & 0.006 & 0.015 & 0.001 & 0.310 & 65.833 \\
\hline ZA16_84_31 & 0.344 & 0.021 & 0.041 & 0.002 & 0.347 & 24.691 \\
\hline
\end{tabular}




\begin{tabular}{|c|c|c|c|c|c|c|}
\hline ZA16_84_32 & 0.335 & 0.021 & 0.048 & 0.002 & 0.213 & 21.008 \\
\hline ZA16_84_33 & 0.659 & 0.079 & 0.048 & 0.002 & 0.284 & 20.747 \\
\hline ZA16_84_34 & 0.274 & 0.014 & 0.032 & 0.002 & 0.776 & 30.960 \\
\hline ZA16_84_35 & 0.329 & 0.008 & 0.047 & 0.001 & 0.214 & 21.093 \\
\hline ZA16_84_36 & 0.310 & 0.010 & 0.041 & 0.001 & 0.442 & 24.396 \\
\hline ZA16_84_37 & 0.393 & 0.018 & 0.044 & 0.001 & 0.308 & 22.779 \\
\hline ZA16_84_38 & 0.336 & 0.013 & 0.048 & 0.001 & 0.299 & 20.833 \\
\hline ZA16_84_39 & 0.343 & 0.012 & 0.046 & 0.001 & 0.227 & 21.834 \\
\hline ZA16_84_40 & 0.475 & 0.031 & 0.048 & 0.002 & 0.250 & 20.704 \\
\hline ZA16_84_41 & 0.530 & 0.042 & 0.048 & 0.002 & 0.649 & 20.661 \\
\hline ZA16_84_42 & 0.479 & 0.026 & 0.046 & 0.001 & 0.225 & 21.882 \\
\hline ZA16_84_43 & 0.650 & 0.055 & 0.047 & 0.002 & 0.449 & 21.231 \\
\hline ZA16_84_44 & 1.340 & 0.300 & 0.056 & 0.005 & 0.913 & 17.762 \\
\hline ZA16_84_45 & 0.400 & 0.022 & 0.047 & 0.002 & 0.274 & 21.459 \\
\hline ZA16_84_46 & 0.356 & 0.015 & 0.047 & 0.001 & 0.368 & 21.277 \\
\hline ZA16_84_47 & 0.398 & 0.026 & 0.047 & 0.002 & 0.230 & 21.413 \\
\hline ZA16_84_48 & 0.569 & 0.055 & 0.049 & 0.002 & 0.550 & 20.576 \\
\hline ZA16_84_49 & 0.336 & 0.014 & 0.046 & 0.002 & 0.502 & 21.978 \\
\hline ZA16_84_50 & 0.338 & 0.014 & 0.046 & 0.002 & 0.334 & 21.786 \\
\hline ZA16_84_51 & 0.346 & 0.018 & 0.046 & 0.002 & 0.784 & 21.739 \\
\hline ZA16_84_53 & 0.328 & 0.016 & 0.045 & 0.002 & 0.520 & 22.371 \\
\hline ZA16_84_54 & 0.347 & 0.015 & 0.048 & 0.002 & 0.403 & 21.008 \\
\hline ZA16_84_55 & 0.374 & 0.029 & 0.049 & 0.002 & 0.234 & 20.450 \\
\hline ZA16_84_56 & 0.333 & 0.017 & 0.047 & 0.002 & 0.291 & 21.277 \\
\hline ZA16_84_57 & 0.335 & 0.026 & 0.046 & 0.002 & 0.191 & 21.834 \\
\hline ZA16_84_58 & 0.368 & 0.022 & 0.049 & 0.001 & 0.066 & 20.243 \\
\hline ZA16_84_59 & 0.345 & 0.019 & 0.048 & 0.001 & 0.062 & 20.704 \\
\hline ZA16_84_60 & 0.341 & 0.016 & 0.047 & 0.001 & 0.007 & 21.186 \\
\hline ZA16_84_61 & 0.350 & 0.016 & 0.048 & 0.001 & 0.113 & 20.661 \\
\hline ZA16_84_62 & 0.577 & 0.060 & 0.050 & 0.002 & 0.402 & 19.960 \\
\hline ZA16_84_63 & 0.341 & 0.018 & 0.047 & 0.001 & 0.177 & 21.186 \\
\hline ZA16_84_64 & 0.455 & 0.024 & 0.048 & 0.001 & 0.252 & 20.964 \\
\hline ZA16_84_65 & 0.330 & 0.014 & 0.046 & 0.001 & 0.134 & 21.678 \\
\hline ZA16_84_66 & 0.392 & 0.017 & 0.048 & 0.001 & 0.004 & 20.704 \\
\hline ZA16_84_67 & 0.349 & 0.015 & 0.047 & 0.001 & 0.225 & 21.169 \\
\hline ZA16_84_68 & 0.623 & 0.061 & 0.051 & 0.001 & 0.436 & 19.763 \\
\hline ZA16_84_69 & 0.368 & 0.023 & 0.047 & 0.001 & 0.323 & 21.277 \\
\hline ZA16_84_70 & 0.351 & 0.026 & 0.047 & 0.001 & 0.510 & 21.209 \\
\hline ZA16_84_71 & 0.367 & 0.013 & 0.049 & 0.001 & 0.030 & 20.462 \\
\hline ZA16_84_72 & 0.355 & 0.014 & 0.048 & 0.001 & 0.250 & 20.921 \\
\hline ZA16_84_73 & 0.509 & 0.035 & 0.051 & 0.002 & 0.287 & 19.646 \\
\hline ZA16_84_74 & 0.699 & 0.048 & 0.051 & 0.001 & 0.010 & 19.724 \\
\hline ZA16_119_1 & 0.687 & 0.016 & 0.023 & 0.001 & 0.562 & 43.459 \\
\hline ZA16_119_2 & 0.330 & 0.011 & 0.027 & 0.001 & 0.504 & 37.736 \\
\hline ZA16_119_3 & 3.080 & 0.300 & 0.060 & 0.003 & 0.868 & 16.722 \\
\hline ZA16_119_4 & 0.344 & 0.010 & 0.047 & 0.001 & 0.333 & 21.102 \\
\hline ZA16_119_5 & 0.774 & 0.073 & 0.051 & 0.002 & 0.444 & 19.763 \\
\hline ZA16_119_6 & 0.351 & 0.012 & 0.047 & 0.001 & 0.296 & 21.427 \\
\hline ZA16_119_7 & 0.330 & 0.016 & 0.048 & 0.001 & 0.107 & 20.833 \\
\hline
\end{tabular}




\begin{tabular}{|c|c|c|c|c|c|c|}
\hline ZA16_119_8 & 0.514 & 0.020 & 0.057 & 0.002 & 0.120 & 17.422 \\
\hline ZA16_119_9 & 0.552 & 0.026 & 0.052 & 0.001 & 0.291 & 19.084 \\
\hline ZA16_119_10 & 0.362 & 0.016 & 0.048 & 0.001 & 0.103 & 20.636 \\
\hline ZA16_119_11 & 0.388 & 0.023 & 0.048 & 0.001 & 0.452 & 20.921 \\
\hline ZA16_119_12 & 0.408 & 0.016 & 0.049 & 0.001 & 0.010 & 20.325 \\
\hline ZA16_119_13 & 3.650 & 0.230 & 0.077 & 0.003 & 0.918 & 12.920 \\
\hline ZA16_119_14 & 0.358 & 0.021 & 0.031 & 0.001 & 0.070 & 32.031 \\
\hline ZA16_119_15 & 0.289 & 0.026 & 0.032 & 0.002 & 0.788 & 31.447 \\
\hline ZA16_119_16 & 0.360 & 0.021 & 0.035 & 0.001 & 0.537 & 28.736 \\
\hline ZA16_119_17 & 0.541 & 0.018 & 0.047 & 0.002 & 0.348 & 21.459 \\
\hline ZA16_119_18 & 0.378 & 0.012 & 0.042 & 0.002 & 0.265 & 23.810 \\
\hline ZA16_119_19 & 0.350 & 0.012 & 0.047 & 0.001 & 0.268 & 21.277 \\
\hline ZA16_119_20 & 0.358 & 0.016 & 0.048 & 0.001 & 0.024 & 20.833 \\
\hline ZA16_119_21 & 0.259 & 0.007 & 0.026 & 0.001 & 0.462 & 38.580 \\
\hline ZA16_119_22 & 0.579 & 0.060 & 0.048 & 0.003 & 0.463 & 21.008 \\
\hline ZA16_119_24 & 0.843 & 0.075 & 0.051 & 0.001 & 0.512 & 19.685 \\
\hline ZA16_119_25 & 2.760 & 0.190 & 0.067 & 0.002 & 0.766 & 14.881 \\
\hline ZA16_119_26 & 0.347 & 0.013 & 0.046 & 0.001 & 0.501 & 21.978 \\
\hline ZA16_119_27 & 0.351 & 0.014 & 0.046 & 0.002 & 0.606 & 21.882 \\
\hline ZA16_119_28 & 0.400 & 0.011 & 0.049 & 0.001 & 0.142 & 20.547 \\
\hline ZA16_119_29 & 0.358 & 0.017 & 0.048 & 0.002 & 0.637 & 21.008 \\
\hline ZA16_119_30 & 0.467 & 0.022 & 0.045 & 0.001 & 0.424 & 22.075 \\
\hline ZA16_119_31 & 0.938 & 0.036 & 0.046 & 0.002 & 0.267 & 21.978 \\
\hline ZA16_119_32 & 0.372 & 0.019 & 0.011 & 0.000 & 0.010 & 93.371 \\
\hline ZA16_119_33 & 0.631 & 0.034 & 0.016 & 0.001 & 0.551 & 61.728 \\
\hline ZA16_119_34 & 0.836 & 0.050 & 0.019 & 0.002 & 0.742 & 53.476 \\
\hline ZA16_119_35 & 0.324 & 0.016 & 0.007 & 0.001 & 0.673 & 152.905 \\
\hline ZA16_119_36 & 0.247 & 0.014 & 0.012 & 0.001 & 0.607 & 81.833 \\
\hline ZA16_119_37 & 0.371 & 0.017 & 0.050 & 0.002 & 0.161 & 20.000 \\
\hline ZA16_119_39 & 0.360 & 0.012 & 0.049 & 0.001 & 0.363 & 20.619 \\
\hline ZA16_119_40 & 0.264 & 0.007 & 0.021 & 0.001 & 0.701 & 47.461 \\
\hline ZA16_119_41 & 0.375 & 0.016 & 0.014 & 0.001 & 0.817 & 69.061 \\
\hline ZA16_119_42 & 0.648 & 0.023 & 0.045 & 0.001 & 0.361 & 22.371 \\
\hline ZA16_119_43 & 0.386 & 0.020 & 0.047 & 0.001 & 0.674 & 21.097 \\
\hline Z_Plesovice_1 & 0.398 & 0.014 & 0.055 & 0.001 & 0.410 & 18.215 \\
\hline Z_Plesovice_2 & 0.411 & 0.013 & 0.055 & 0.001 & 0.257 & 18.315 \\
\hline Z_Plesovice_3 & 0.392 & 0.009 & 0.054 & 0.001 & 0.401 & 18.460 \\
\hline Z_Plesovice_4 & 0.399 & 0.009 & 0.053 & 0.001 & 0.293 & 18.847 \\
\hline Z_Plesovice_5 & 0.410 & 0.014 & 0.057 & 0.002 & 0.584 & 17.699 \\
\hline Z_Plesovice_6 & 0.396 & 0.009 & 0.053 & 0.001 & 0.412 & 18.720 \\
\hline Z_Plesovice_7 & 0.409 & 0.011 & 0.056 & 0.002 & 0.357 & 17.953 \\
\hline Z_Plesovice_8 & 0.405 & 0.013 & 0.057 & 0.001 & 0.323 & 17.575 \\
\hline Z_Plesovice_9 & 0.399 & 0.010 & 0.054 & 0.001 & 0.371 & 18.352 \\
\hline Z_Plesovice_10 & 0.404 & 0.011 & 0.056 & 0.002 & 0.261 & 17.825 \\
\hline Z_Plesovice_11 & 0.406 & 0.013 & 0.056 & 0.001 & 0.418 & 17.953 \\
\hline Z_Plesovice_12 & 0.392 & 0.010 & 0.054 & 0.001 & 0.387 & 18.362 \\
\hline Z_Plesovice_13 & 0.412 & 0.011 & 0.056 & 0.002 & 0.518 & 17.825 \\
\hline Z_Plesovice_14 & 0.400 & 0.012 & 0.055 & 0.001 & 0.296 & 18.282 \\
\hline Z_Plesovice_15 & 0.408 & 0.016 & 0.055 & 0.001 & 0.571 & 18.051 \\
\hline
\end{tabular}




$\begin{array}{lllllll}\text { Z_Plesovice_16 } & 0.404 & 0.010 & 0.056 & 0.002 & 0.410 & 17.762 \\ \text { Z_Plesovice_17 } & 0.412 & 0.014 & 0.055 & 0.001 & 0.330 & 18.116 \\ \text { Z_Plesovice_18 } & 0.401 & 0.009 & 0.054 & 0.001 & 0.454 & 18.522 \\ \text { Z_Plesovice_19 } & 0.407 & 0.012 & 0.056 & 0.001 & 0.434 & 17.762 \\ \text { Z_Plesovice_20 } & 0.412 & 0.012 & 0.055 & 0.001 & 0.476 & 18.083 \\ & & & & & & \\ \text { Z_Temora2_1 } & 0.509 & 0.026 & 0.067 & 0.002 & 0.075 & 14.881 \\ \text { Z_Temora2_2 } & 0.488 & 0.030 & 0.066 & 0.002 & 0.010 & 15.198 \\ \text { Z_Temora2_3 } & 0.507 & 0.025 & 0.067 & 0.002 & 0.224 & 15.038 \\ \text { Z_Temora244 } & 0.499 & 0.025 & 0.065 & 0.001 & 0.102 & 15.337 \\ \text { Z_Temora2_5 } & 0.507 & 0.026 & 0.067 & 0.001 & 0.169 & 14.903 \\ \text { Z_Temora2_6 } & 0.515 & 0.027 & 0.068 & 0.002 & 0.127 & 14.663 \\ \text { Z_Temora2_7 } & 0.521 & 0.021 & 0.068 & 0.001 & 0.095 & 14.620 \\ \text { Z_Temora2_9 } & 0.511 & 0.018 & 0.066 & 0.001 & 0.157 & 15.221 \\ \text { Z_Temora2_10 } & 0.512 & 0.020 & 0.068 & 0.001 & 0.281 & 14.620 \\ \text { Z_Temora2_11 } & 0.518 & 0.023 & 0.067 & 0.001 & 0.259 & 14.948 \\ \text { Z_Temora2_12 } & 0.503 & 0.028 & 0.068 & 0.001 & 0.158 & 14.641 \\ \text { Z_Temora2_13 } & 0.493 & 0.023 & 0.065 & 0.002 & 0.119 & 15.361 \\ \text { Z_Temora2_14 } & 0.488 & 0.028 & 0.067 & 0.002 & 0.260 & 14.925 \\ \text { Z_Temora2_15 } & 0.500 & 0.029 & 0.066 & 0.001 & 0.010 & 15.106 \\ \text { Z_Temora2_16 } & 0.506 & 0.032 & 0.068 & 0.002 & 0.134 & 14.815 \\ \text { Z_Temora2_17 } & 0.510 & 0.025 & 0.065 & 0.001 & 0.348 & 15.337 \\ \text { Z_Temora2_18 } & 0.513 & 0.022 & 0.068 & 0.002 & 0.090 & 14.706 \\ \text { Z_Temora2_20 } & 0.516 & 0.026 & 0.065 & 0.002 & 0.010 & 15.385\end{array}$


Page 144 of 203 


\begin{tabular}{|c|c|c|c|c|c|c|}
\hline Error $2 \sigma$ & ${ }^{207} \mathrm{~Pb} /{ }^{206} \mathrm{~Pb}$ & Error $2 \sigma$ & Jrrelation_38 & alAge ${ }^{206} \mathrm{~Pb} /{ }^{23}$ & Error $2 \sigma$ & FinalAge ${ }^{207} \mathrm{~Pb}$ \\
\hline 0.222 & 0.056 & 0.003 & 0.297 & 525.304 & 9.900 & 515.837 \\
\hline 0.209 & 0.056 & 0.003 & 0.335 & 524.116 & 9.282 & 514.614 \\
\hline 0.269 & 0.058 & 0.003 & 0.119 & 519.954 & 11.761 & 523.142 \\
\hline 0.271 & 0.055 & 0.003 & 0.217 & 518.765 & 11.762 & 504.780 \\
\hline 0.253 & 0.057 & 0.003 & 0.454 & 521.738 & 11.140 & 510.937 \\
\hline 0.243 & 0.057 & 0.003 & 0.481 & 518.170 & 10.524 & 520.104 \\
\hline 0.227 & 0.057 & 0.003 & 0.193 & 519.360 & 9.904 & 515.225 \\
\hline 0.233 & 0.058 & 0.003 & 0.645 & 512.814 & 9.909 & 515.225 \\
\hline 0.228 & 0.058 & 0.003 & 0.469 & 518.765 & 9.905 & 520.712 \\
\hline 0.259 & 0.057 & 0.003 & 0.196 & 516.385 & 11.145 & 523.142 \\
\hline 0.215 & 0.059 & 0.003 & 0.458 & 517.575 & 9.287 & 527.982 \\
\hline 0.233 & 0.057 & 0.003 & 0.424 & 528.868 & 10.516 & 529.189 \\
\hline 0.280 & 0.058 & 0.003 & 0.464 & 535.398 & 12.983 & 544.746 \\
\hline 0.234 & 0.057 & 0.003 & 0.318 & 527.087 & 10.517 & 521.928 \\
\hline 0.223 & 0.058 & 0.003 & 0.285 & 524.116 & 9.901 & 518.887 \\
\hline 0.231 & 0.059 & 0.004 & 0.404 & 530.056 & 10.515 & 545.340 \\
\hline 0.236 & 0.057 & 0.003 & 0.111 & 539.549 & 11.125 & 529.189 \\
\hline 0.256 & 0.057 & 0.003 & 0.208 & 532.431 & 11.749 & 526.170 \\
\hline 0.298 & 0.057 & 0.004 & 0.159 & 519.360 & 12.999 & 527.982 \\
\hline 0.230 & 0.056 & 0.003 & 0.366 & 531.837 & 10.513 & 523.748 \\
\hline 0.625 & 0.114 & 0.007 & 0.154 & 308.379 & 9.440 & 579.189 \\
\hline 0.417 & 0.051 & 0.002 & 0.322 & 299.093 & 5.920 & 300.953 \\
\hline 0.483 & 0.052 & 0.002 & 0.248 & 286.835 & 6.304 & 291.092 \\
\hline 0.440 & 0.060 & 0.004 & 0.250 & 300.385 & 6.297 & 338.015 \\
\hline 0.485 & 0.075 & 0.006 & -0.029 & 299.770 & 6.927 & 400.813 \\
\hline 4.217 & 0.069 & 0.003 & -0.196 & 98.518 & 6.397 & 140.675 \\
\hline 0.442 & 0.051 & 0.003 & 0.268 & 296.754 & 6.173 & 292.615 \\
\hline 0.523 & 0.048 & 0.004 & 0.516 & 301.616 & 7.556 & 281.133 \\
\hline 0.485 & 0.051 & 0.002 & 0.065 & 299.770 & 6.927 & 297.172 \\
\hline 0.455 & 0.050 & 0.002 & 0.339 & 295.461 & 6.300 & 287.273 \\
\hline 0.440 & 0.053 & 0.003 & 0.386 & 300.385 & 6.297 & 306.975 \\
\hline 0.618 & 0.055 & 0.004 & 0.317 & 299.770 & 8.817 & 309.224 \\
\hline 0.598 & 0.072 & 0.005 & -0.514 & 315.136 & 9.435 & 421.135 \\
\hline 0.670 & 0.054 & 0.004 & 0.297 & 288.068 & 8.825 & 298.686 \\
\hline 0.519 & 0.060 & 0.002 & 0.175 & 302.846 & 7.555 & 335.100 \\
\hline 0.732 & 0.091 & 0.004 & 0.201 & 303.461 & 10.703 & 492.976 \\
\hline 5.443 & 0.091 & 0.010 & 0.925 & 106.132 & 9.590 & 188.505 \\
\hline 0.679 & 0.074 & 0.004 & 0.507 & 286.218 & 8.826 & 388.422 \\
\hline 0.664 & 0.093 & 0.007 & 0.423 & 318.204 & 10.691 & 517.668 \\
\hline 0.781 & 0.094 & 0.007 & -0.179 & 318.204 & 12.577 & 513.390 \\
\hline 0.483 & 0.058 & 0.003 & 0.051 & 300.385 & 6.927 & 327.038 \\
\hline 0.425 & 0.052 & 0.002 & 0.532 & 304.015 & 6.233 & 303.216 \\
\hline 0.460 & 0.095 & 0.007 & -0.447 & 307.765 & 6.923 & 509.709 \\
\hline 0.428 & 0.060 & 0.002 & 0.388 & 302.723 & 6.233 & 343.098 \\
\hline 0.453 & 0.069 & 0.003 & 0.562 & 296.077 & 6.300 & 373.772 \\
\hline 0.468 & 0.283 & 0.013 & -0.232 & 408.382 & 12.489 & 1274.931 \\
\hline 2.904 & 0.076 & 0.004 & 0.746 & 97.185 & 4.287 & 152.452 \\
\hline 1.280 & 0.062 & 0.004 & 0.426 & 255.931 & 13.271 & 300.198 \\
\hline
\end{tabular}




\begin{tabular}{|c|c|c|c|c|c|c|}
\hline 0.750 & 0.051 & 0.003 & 0.182 & 299.770 & 10.706 & 293.376 \\
\hline 0.861 & 0.099 & 0.010 & -0.006 & 303.461 & 12.592 & 514.002 \\
\hline 2.013 & 0.062 & 0.002 & 0.574 & 204.927 & 13.323 & 245.887 \\
\hline 0.400 & 0.051 & 0.002 & 0.514 & 298.601 & 5.668 & 288.496 \\
\hline 0.548 & 0.055 & 0.002 & 0.171 & 258.967 & 5.812 & 274.181 \\
\hline 0.726 & 0.065 & 0.004 & 0.336 & 276.962 & 8.832 & 336.559 \\
\hline 0.477 & 0.050 & 0.002 & 0.335 & 302.231 & 6.926 & 294.136 \\
\hline 0.524 & 0.053 & 0.002 & 0.471 & 288.684 & 6.933 & 299.442 \\
\hline 0.729 & 0.071 & 0.005 & 0.263 & 304.076 & 10.702 & 394.637 \\
\hline 1.025 & 0.081 & 0.005 & 0.044 & 304.691 & 15.109 & 431.810 \\
\hline 0.670 & 0.076 & 0.004 & 0.319 & 288.068 & 8.825 & 397.387 \\
\hline 0.902 & 0.095 & 0.007 & 0.144 & 296.693 & 12.598 & 508.479 \\
\hline 1.420 & 0.164 & 0.022 & -0.685 & 353.085 & 28.222 & 863.229 \\
\hline 0.829 & 0.064 & 0.003 & 0.277 & 293.614 & 11.341 & 341.648 \\
\hline 0.543 & 0.055 & 0.002 & 0.385 & 296.077 & 7.559 & 309.224 \\
\hline 0.688 & 0.062 & 0.004 & 0.246 & 294.230 & 9.451 & 340.197 \\
\hline 1.016 & 0.087 & 0.007 & 0.008 & 305.921 & 15.107 & 457.368 \\
\hline 0.773 & 0.054 & 0.002 & 0.428 & 286.835 & 10.086 & 294.136 \\
\hline 0.712 & 0.055 & 0.002 & 0.505 & 289.301 & 9.454 & 295.655 \\
\hline 0.898 & 0.055 & 0.003 & 0.288 & 289.917 & 11.975 & 301.708 \\
\hline 0.901 & 0.054 & 0.003 & 0.244 & 281.900 & 11.352 & 288.038 \\
\hline 0.794 & 0.053 & 0.002 & 0.479 & 299.770 & 11.336 & 302.462 \\
\hline 0.753 & 0.054 & 0.004 & 0.306 & 307.765 & 11.329 & 322.614 \\
\hline 1.086 & 0.053 & 0.003 & 0.150 & 296.077 & 15.119 & 291.854 \\
\hline 1.001 & 0.051 & 0.005 & 0.363 & 288.684 & 13.237 & 293.376 \\
\hline 0.451 & 0.055 & 0.003 & 0.287 & 310.837 & 6.921 & 318.170 \\
\hline 0.429 & 0.052 & 0.003 & 0.332 & 304.076 & 6.296 & 300.953 \\
\hline 0.628 & 0.053 & 0.003 & 0.460 & 297.308 & 8.818 & 297.929 \\
\hline 0.470 & 0.053 & 0.003 & 0.336 & 304.691 & 6.925 & 304.721 \\
\hline 0.876 & 0.088 & 0.009 & 0.066 & 315.136 & 13.838 & 462.532 \\
\hline 0.494 & 0.052 & 0.003 & 0.265 & 297.308 & 6.929 & 297.929 \\
\hline 0.483 & 0.069 & 0.004 & 0.203 & 300.385 & 6.927 & 380.775 \\
\hline 0.432 & 0.051 & 0.002 & 0.264 & 290.718 & 5.798 & 289.566 \\
\hline 0.472 & 0.058 & 0.003 & 0.346 & 304.076 & 6.925 & 335.829 \\
\hline 0.435 & 0.053 & 0.002 & 0.205 & 297.554 & 6.110 & 303.969 \\
\hline 0.547 & 0.092 & 0.009 & -0.151 & 318.204 & 8.804 & 491.726 \\
\hline 0.634 & 0.056 & 0.003 & -0.045 & 296.077 & 8.819 & 318.170 \\
\hline 0.355 & 0.053 & 0.003 & -0.287 & 297.000 & 4.976 & 305.473 \\
\hline 0.322 & 0.054 & 0.002 & 0.342 & 307.580 & 4.846 & 317.428 \\
\hline 0.481 & 0.054 & 0.002 & 0.303 & 301.001 & 6.927 & 308.475 \\
\hline 0.579 & 0.075 & 0.005 & 0.140 & 320.045 & 9.432 & 417.776 \\
\hline 0.428 & 0.100 & 0.007 & 0.364 & 318.818 & 6.917 & 538.193 \\
\hline 1.757 & 0.220 & 0.008 & 0.785 & 146.651 & 5.927 & 530.996 \\
\hline 1.125 & 0.090 & 0.003 & 0.495 & 168.606 & 5.026 & 289.566 \\
\hline 0.839 & 0.362 & 0.017 & -0.476 & 374.409 & 18.783 & 1427.727 \\
\hline 0.423 & 0.055 & 0.002 & 0.395 & 298.478 & 5.983 & 300.198 \\
\hline 0.586 & 0.107 & 0.009 & -0.355 & 318.204 & 9.433 & 582.055 \\
\hline 0.367 & 0.056 & 0.002 & 0.160 & 294.045 & 5.040 & 305.473 \\
\hline 0.608 & 0.050 & 0.003 & 0.419 & 302.231 & 8.815 & 289.566 \\
\hline
\end{tabular}




\begin{tabular}{|c|c|c|c|c|c|c|}
\hline 0.486 & 0.065 & 0.003 & 0.298 & 359.794 & 10.029 & 421.135 \\
\hline 0.364 & 0.076 & 0.004 & 0.075 & 329.239 & 6.283 & 446.306 \\
\hline 0.417 & 0.054 & 0.003 & 0.345 & 305.060 & 6.169 & 313.707 \\
\hline 0.481 & 0.059 & 0.004 & -0.086 & 301.001 & 6.927 & 332.907 \\
\hline 0.454 & 0.061 & 0.003 & 0.424 & 309.608 & 6.922 & 347.434 \\
\hline 0.451 & 0.339 & 0.011 & -0.569 & 480.585 & 16.765 & 1560.509 \\
\hline 1.016 & 0.084 & 0.006 & 0.342 & 198.179 & 6.284 & 310.720 \\
\hline 1.879 & 0.065 & 0.003 & -0.092 & 201.804 & 12.057 & 257.772 \\
\hline 1.156 & 0.074 & 0.003 & 0.299 & 220.520 & 8.871 & 312.215 \\
\hline 0.875 & 0.084 & 0.003 & 0.536 & 293.614 & 11.971 & 439.084 \\
\hline 0.850 & 0.068 & 0.003 & 0.583 & 265.218 & 9.472 & 325.565 \\
\hline 0.543 & 0.054 & 0.002 & 0.507 & 296.077 & 7.559 & 304.721 \\
\hline 0.564 & 0.054 & 0.003 & 0.444 & 302.231 & 8.185 & 310.720 \\
\hline 1.131 & 0.072 & 0.003 & 0.542 & 164.962 & 4.837 & 234.022 \\
\hline 1.148 & 0.091 & 0.009 & -0.060 & 299.770 & 16.374 & 463.819 \\
\hline 0.426 & 0.122 & 0.009 & -0.271 & 319.431 & 6.917 & 620.800 \\
\hline 0.487 & 0.296 & 0.015 & -0.321 & 419.264 & 13.726 & 1344.793 \\
\hline 0.580 & 0.055 & 0.002 & 0.308 & 286.835 & 7.565 & 302.462 \\
\hline 0.766 & 0.057 & 0.002 & 0.100 & 288.068 & 10.086 & 305.473 \\
\hline 0.329 & 0.060 & 0.002 & 0.452 & 306.351 & 4.910 & 341.648 \\
\hline 0.706 & 0.055 & 0.002 & 0.177 & 299.770 & 10.076 & 310.720 \\
\hline 0.634 & 0.077 & 0.004 & 0.226 & 285.601 & 8.196 & 389.115 \\
\hline 0.725 & 0.152 & 0.007 & 0.437 & 286.835 & 9.456 & 671.835 \\
\hline 2.964 & 0.253 & 0.017 & 0.523 & 68.674 & 2.180 & 321.135 \\
\hline 3.810 & 0.296 & 0.019 & 0.780 & 103.595 & 6.395 & 496.719 \\
\hline 5.147 & 0.326 & 0.024 & 0.801 & 119.435 & 11.496 & 616.936 \\
\hline 12.391 & 0.359 & 0.022 & 0.830 & 42.022 & 3.405 & 284.975 \\
\hline 4.018 & 0.149 & 0.006 & 0.326 & 78.298 & 3.844 & 224.136 \\
\hline 0.760 & 0.055 & 0.002 & -0.065 & 314.522 & 11.952 & 320.394 \\
\hline 0.425 & 0.055 & 0.002 & 0.165 & 305.306 & 6.295 & 312.215 \\
\hline 1.892 & 0.092 & 0.003 & 0.696 & 134.415 & 5.359 & 237.564 \\
\hline 4.102 & 0.188 & 0.007 & 0.762 & 92.675 & 5.504 & 323.353 \\
\hline 0.500 & 0.106 & 0.004 & 0.190 & 281.900 & 6.306 & 507.247 \\
\hline 0.490 & 0.060 & 0.002 & -0.379 & 298.539 & 6.928 & 331.443 \\
\hline 0.464 & 0.053 & 0.002 & 0.541 & 344.535 & 8.786 & 340.197 \\
\hline 0.436 & 0.053 & 0.002 & 0.412 & 342.701 & 8.160 & 349.595 \\
\hline 0.317 & 0.052 & 0.001 & 0.296 & 340.072 & 5.838 & 335.465 \\
\hline 0.284 & 0.053 & 0.001 & 0.376 & 333.281 & 5.025 & 340.850 \\
\hline 0.470 & 0.053 & 0.002 & 0.231 & 354.305 & 9.406 & 348.875 \\
\hline 0.322 & 0.053 & 0.001 & 0.389 & 335.484 & 5.778 & 338.525 \\
\hline 0.483 & 0.053 & 0.002 & 0.666 & 349.422 & 9.410 & 348.155 \\
\hline 0.432 & 0.053 & 0.002 & 0.489 & 356.745 & 8.778 & 345.268 \\
\hline 0.320 & 0.053 & 0.001 & 0.372 & 342.029 & 5.963 & 340.923 \\
\hline 0.477 & 0.053 & 0.002 & 0.643 & 351.864 & 9.408 & 344.545 \\
\hline 0.419 & 0.054 & 0.002 & 0.384 & 349.422 & 8.155 & 345.991 \\
\hline 0.283 & 0.052 & 0.001 & 0.248 & 341.846 & 5.273 & 335.829 \\
\hline 0.477 & 0.053 & 0.001 & 0.550 & 351.864 & 9.408 & 350.314 \\
\hline 0.334 & 0.053 & 0.002 & 0.481 & 343.313 & 6.276 & 341.648 \\
\hline 0.358 & 0.054 & 0.002 & 0.223 & 347.590 & 6.902 & 347.434 \\
\hline
\end{tabular}




\begin{tabular}{|c|c|c|c|c|c|c|}
\hline 0.473 & 0.053 & 0.001 & 0.606 & 353.085 & 9.407 & 344.256 \\
\hline 0.459 & 0.054 & 0.002 & 0.396 & 346.368 & 8.785 & 350.314 \\
\hline 0.257 & 0.054 & 0.001 & 0.183 & 338.972 & 4.709 & 342.228 \\
\hline 0.410 & 0.052 & 0.002 & 0.369 & 353.085 & 8.153 & 346.712 \\
\hline 0.425 & 0.054 & 0.002 & 0.456 & 346.979 & 8.157 & 350.314 \\
\hline 0.421 & 0.055 & 0.003 & 0.369 & 419.264 & 11.854 & 417.776 \\
\hline 0.370 & 0.054 & 0.004 & 0.515 & 410.802 & 9.989 & 403.547 \\
\hline 0.362 & 0.055 & 0.003 & 0.224 & 415.035 & 9.986 & 416.430 \\
\hline 0.329 & 0.056 & 0.003 & 0.324 & 407.172 & 8.743 & 411.025 \\
\hline 0.267 & 0.055 & 0.003 & 0.272 & 418.660 & 7.487 & 416.430 \\
\hline 0.344 & 0.055 & 0.003 & 0.374 & 425.302 & 9.978 & 421.806 \\
\hline 0.299 & 0.056 & 0.002 & 0.419 & 426.509 & 8.730 & 425.819 \\
\hline 0.278 & 0.056 & 0.002 & 0.308 & 410.197 & 7.492 & 419.121 \\
\hline 0.278 & 0.054 & 0.002 & 0.207 & 426.509 & 8.106 & 419.793 \\
\hline 0.290 & 0.056 & 0.002 & 0.158 & 417.452 & 8.112 & 423.814 \\
\hline 0.279 & 0.053 & 0.003 & 0.182 & 425.906 & 8.107 & 413.731 \\
\hline 0.354 & 0.056 & 0.003 & 0.358 & 406.567 & 9.368 & 406.953 \\
\hline 0.356 & 0.054 & 0.003 & 0.082 & 418.056 & 9.983 & 403.547 \\
\hline 0.297 & 0.055 & 0.004 & 0.358 & 413.221 & 8.115 & 411.702 \\
\hline 0.329 & 0.055 & 0.003 & 0.196 & 421.076 & 9.357 & 415.756 \\
\hline 0.282 & 0.057 & 0.003 & 0.090 & 407.172 & 7.494 & 418.449 \\
\hline 0.324 & 0.056 & 0.003 & 0.370 & 424.095 & 9.355 & 420.464 \\
\hline 0.355 & 0.056 & 0.003 & 0.429 & 405.962 & 9.368 & 422.476 \\
\hline
\end{tabular}




\begin{tabular}{|c|c|c|}
\hline $\operatorname{ror} 2 \sigma$ & FinalAge ${ }^{207} \mathrm{~Pb}$ & Error $2 \sigma$ \\
\hline 23.376 & 452.368 & 21.003 \\
\hline 24.171 & 464.219 & 23.087 \\
\hline 25.614 & 529.762 & 27.401 \\
\hline 28.217 & 428.402 & 23.972 \\
\hline 21.875 & 499.252 & 23.566 \\
\hline 24.878 & 491.533 & 25.870 \\
\hline 23.384 & 491.533 & 25.008 \\
\hline 24.163 & 537.299 & 30.465 \\
\hline 23.315 & 522.189 & 26.200 \\
\hline 26.390 & 499.252 & 25.312 \\
\hline 23.999 & 578.128 & 30.223 \\
\hline 24.757 & 503.098 & 27.218 \\
\hline 26.086 & 544.801 & 27.986 \\
\hline 22.524 & 503.098 & 23.706 \\
\hline 24.894 & 529.762 & 26.488 \\
\hline 30.680 & 563.402 & 35.392 \\
\hline 28.626 & 499.252 & 26.185 \\
\hline 30.997 & 487.660 & 29.140 \\
\hline 34.838 & 503.098 & 33.364 \\
\hline 27.157 & 456.328 & 23.589 \\
\hline 36.152 & 1859.376 & 106.297 \\
\hline 12.213 & 258.809 & 10.574 \\
\hline 11.398 & 302.904 & 12.139 \\
\hline 19.682 & 603.575 & 38.226 \\
\hline 28.156 & 1068.502 & 82.631 \\
\hline 8.993 & 904.708 & 39.221 \\
\hline 13.141 & 249.844 & 12.687 \\
\hline 17.626 & 94.333 & 6.893 \\
\hline 12.236 & 227.214 & 10.756 \\
\hline 12.299 & 190.338 & 9.155 \\
\hline 15.653 & 311.581 & 16.586 \\
\hline 16.504 & 404.072 & 25.808 \\
\hline 36.050 & 988.765 & 71.312 \\
\hline 18.340 & 379.369 & 25.198 \\
\hline 13.713 & 585.440 & 17.711 \\
\hline 26.818 & 1454.950 & 55.715 \\
\hline 9.240 & 1446.603 & 158.967 \\
\hline 16.670 & 1044.192 & 50.730 \\
\hline 34.252 & 1496.011 & 105.714 \\
\hline 44.473 & 1502.075 & 112.215 \\
\hline 18.073 & 533.535 & 27.549 \\
\hline 10.456 & 298.548 & 12.558 \\
\hline 40.652 & 1518.127 & 106.028 \\
\hline 8.535 & 607.177 & 18.185 \\
\hline 15.119 & 895.748 & 44.202 \\
\hline 76.191 & 3379.560 & 155.245 \\
\hline 5.646 & 1097.702 & 59.140 \\
\hline 3.326 & 670.656 & 46.5 \\
\hline
\end{tabular}

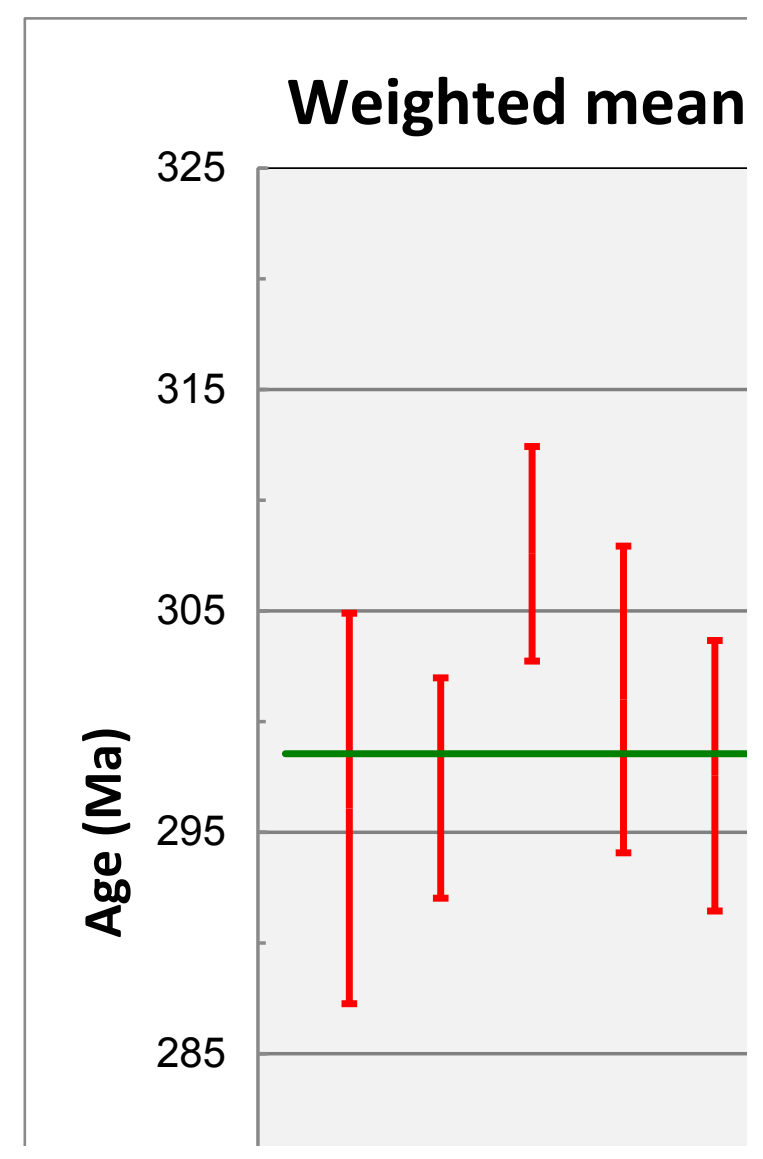




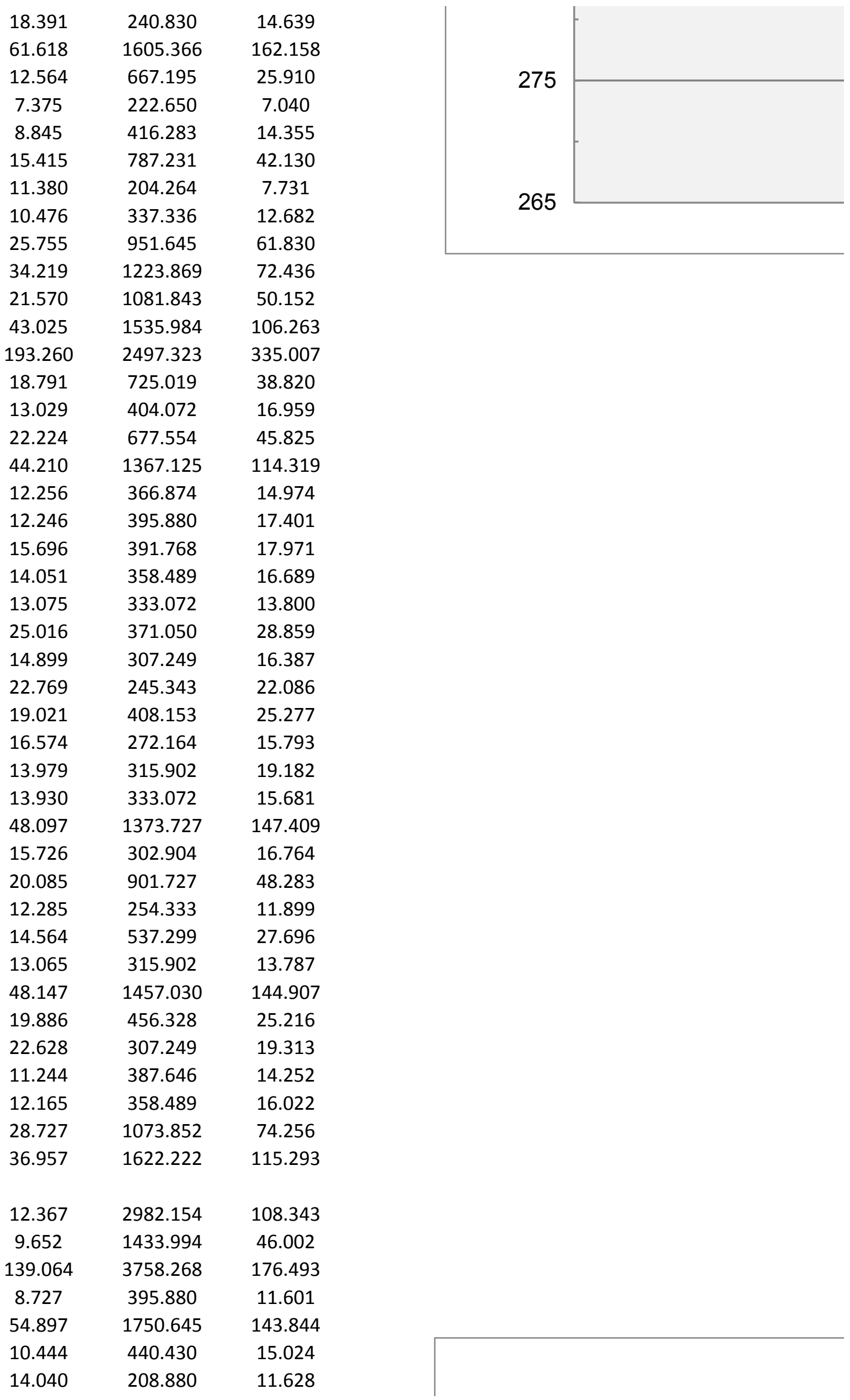




$\begin{array}{ccc}16.387 & 784.017 & 30.016 \\ 21.022 & 1102.953 & 50.594 \\ 13.865 & 375.215 & 18.726 \\ 19.734 & 578.128 & 34.122 \\ 13.625 & 642.756 & 29.455 \\ 98.333 & 3658.268 & 118.705 \\ 18.227 & 1283.261 & 87.495 \\ 23.191 & 761.336 & 37.713 \\ 18.213 & 1027.771 & 46.145 \\ 14.609 & 1283.261 & 52.190 \\ 10.335 & 853.240 & 36.658 \\ 10.448 & 375.215 & 13.871 \\ 13.887 & 350.061 & 18.321 \\ 6.681 & 985.941 & 38.342 \\ 48.064 & 1452.868 & 144.809 \\ 55.231 & 1979.834 & 153.046 \\ 92.576 & 3449.416 & 174.801 \\ 11.331 & 420.333 & 13.707 \\ 12.184 & 483.777 & 19.590 \\ 9.395 & 603.575 & 19.113 \\ 14.755 & 404.072 & 16.959 \\ 18.331 & 1108.185 & 50.701 \\ 25.785 & 2373.031 & 102.769 \\ 16.402 & 3203.637 & 215.264 \\ 26.765 & 3449.416 & 221.415 \\ 36.898 & 3598.375 & 264.911 \\ 14.073 & 3745.628 & 229.537 \\ 12.704 & 2339.070 & 100.201 \\ 14.681 & 404.072 & 15.485 \\ 10.407 & 391.768 & 12.939 \\ 6.399 & 1467.386 & 43.065 \\ 13.796 & 2722.990 & 97.146 \\ 18.004 & 1738.633 & 58.826 \\ 17.173 & 596.346 & 23.934 \\ & & \\ 11.967 & 311.581 & 10.070 \\ 11.058 & 337.336 & 12.048 \\ 7.798 & 276.591 & 7.475 \\ 7.947 & 345.831 & 8.419 \\ 11.913 & 315.902 & 9.591 \\ 7.785 & 337.336 & 7.609 \\ 9.364 & 341.589 & 10.254 \\ 11.083 & 320.212 & 11.523 \\ 8.544 & 311.581 & 7.108 \\ 9.381 & 333.072 & 11.291 \\ 11.079 & 350.061 & 11.123 \\ 8.567 & 298.548 & 6.850 \\ 9.353 & 333.072 & 8.782 \\ 10.249 & 333.072 & 10.036 \\ 13.625 & 383.513 & 13.419\end{array}$

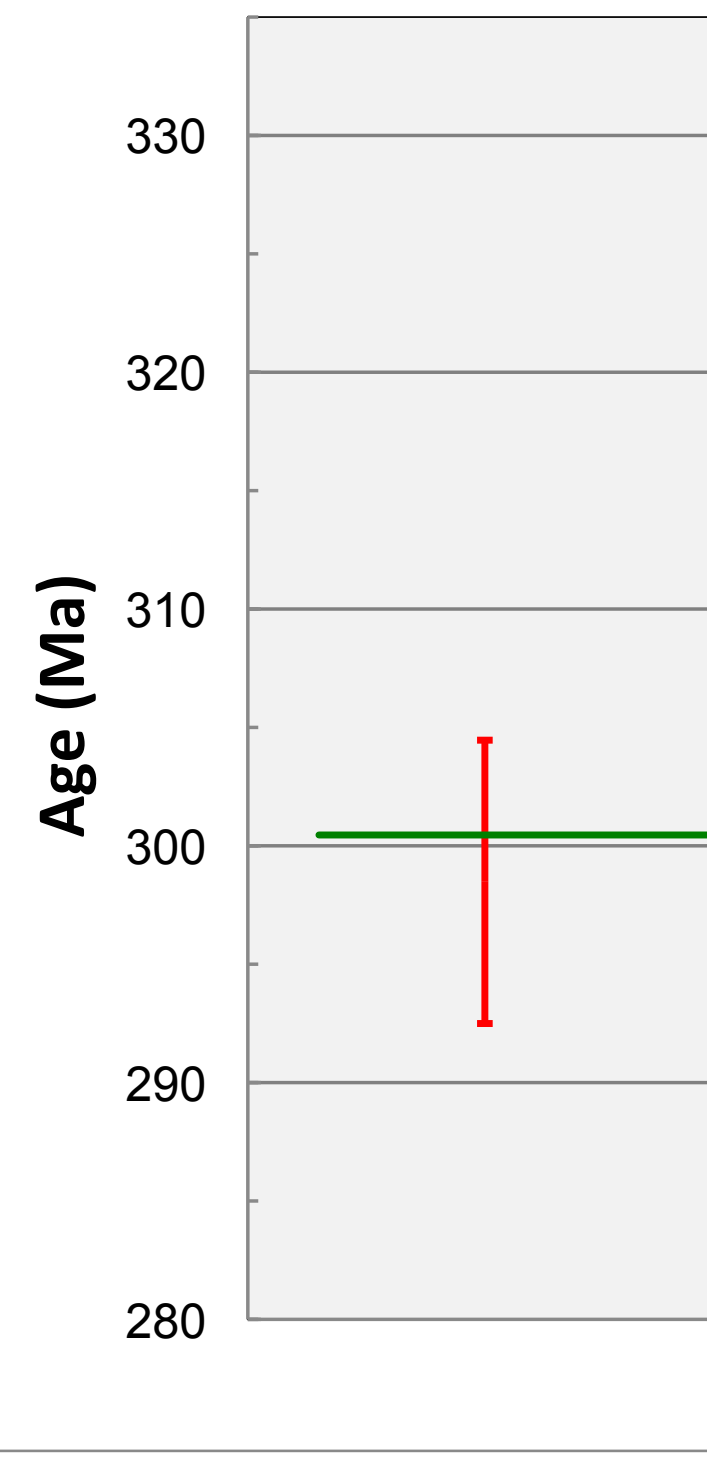




$\begin{array}{ccc}8.359 & 333.072 & 8.782 \\ 11.904 & 383.513 & 14.126 \\ 7.856 & 366.874 & 8.168 \\ 10.222 & 289.801 & 8.344 \\ 10.203 & 383.513 & 12.713 \\ & & \\ 21.340 & 408.153 & 23.047 \\ 24.808 & 366.874 & 25.865 \\ 20.534 & 408.153 & 20.073 \\ 20.592 & 432.421 & 22.595 \\ 21.355 & 399.981 & 19.743 \\ 22.114 & 404.072 & 22.121 \\ 17.164 & 432.421 & 17.920 \\ 14.764 & 444.419 & 15.929 \\ 16.398 & 366.874 & 14.294 \\ 18.818 & 460.278 & 19.656 \\ 23.031 & 341.589 & 19.226 \\ 18.986 & 436.431 & 21.194 \\ 23.154 & 362.687 & 20.898 \\ 23.879 & 408.153 & 28.251 \\ 26.293 & 412.223 & 25.483 \\ 20.512 & 499.252 & 24.439 \\ 18.032 & 436.431 & 21.194 \\ 21.288 & 464.219 & 23.087\end{array}$


Page 154 of 203 


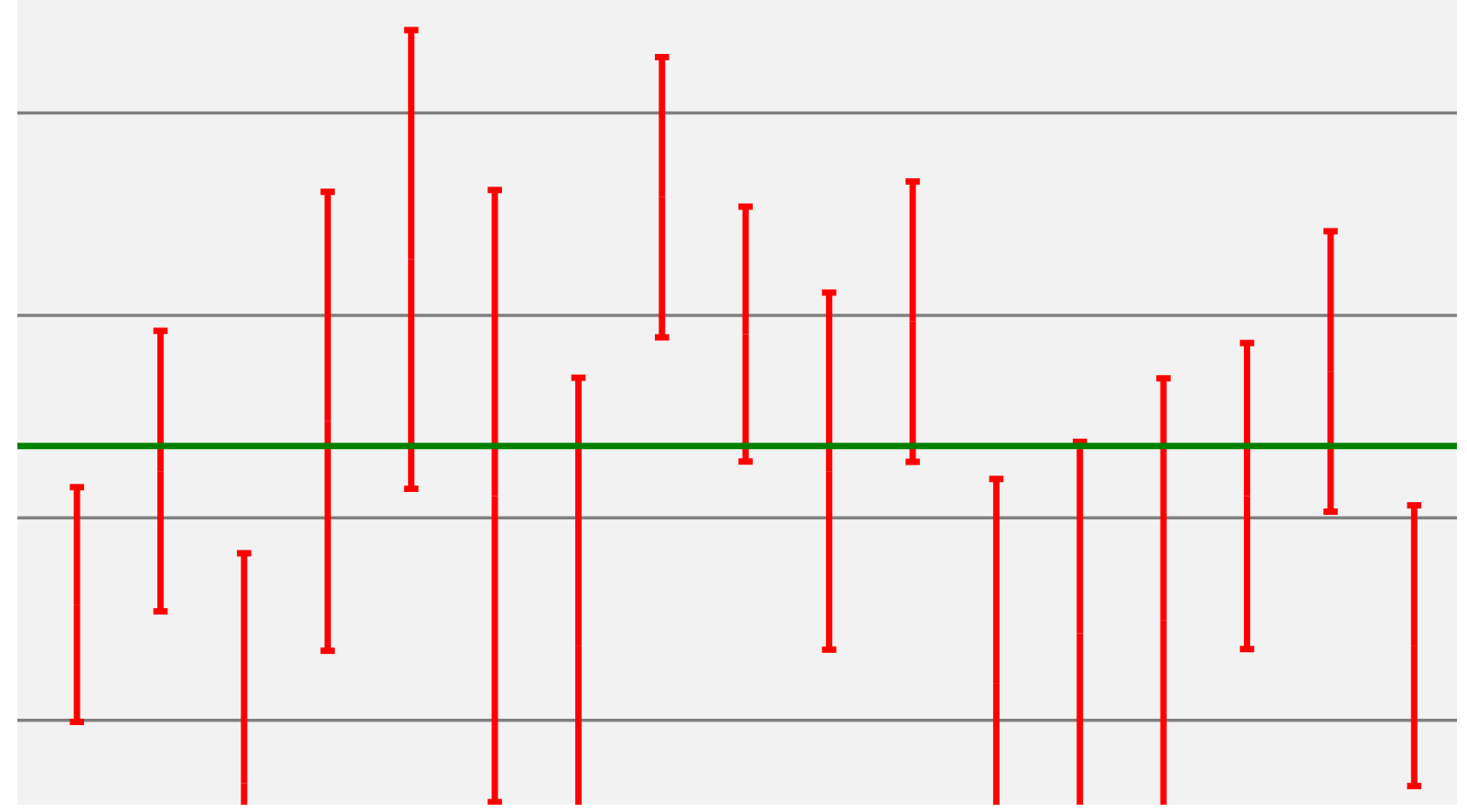




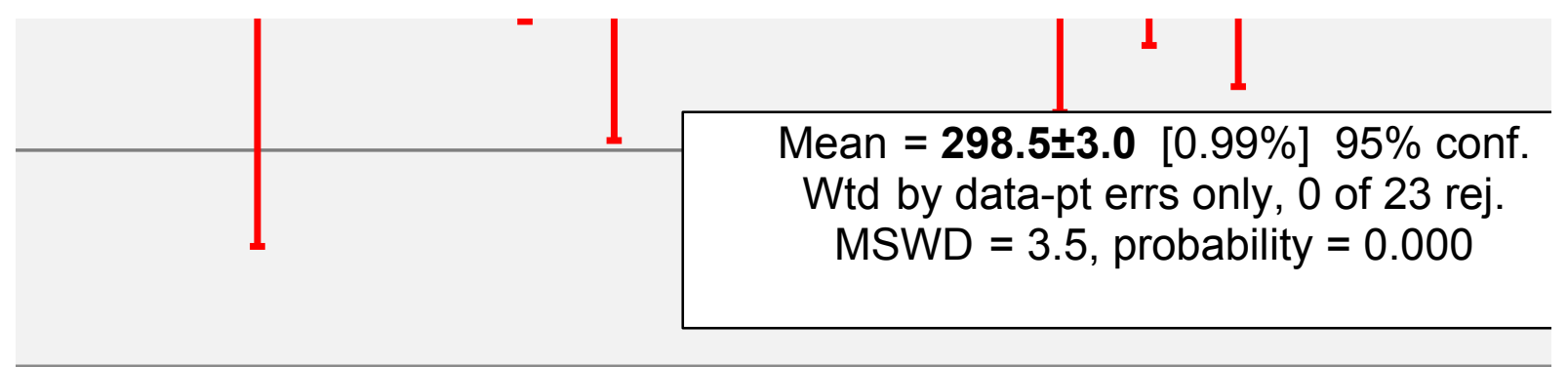

Analyses

Weighted mean age - zircon ZA16-119 


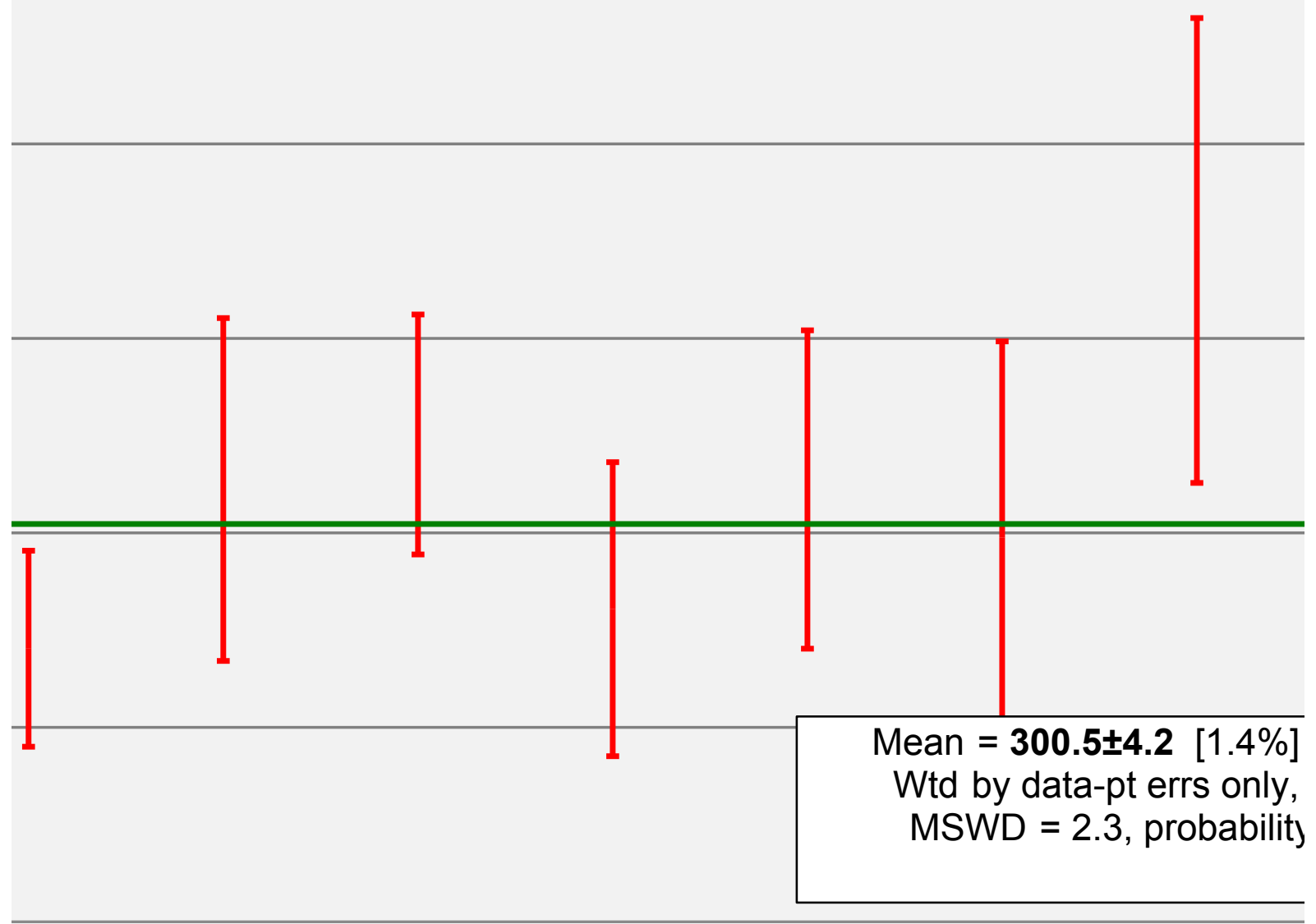

Analyses 
Page 158 of 203 
; are $2 \sigma$

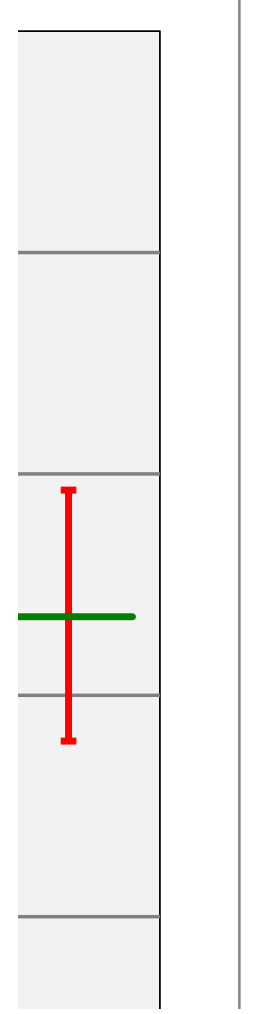




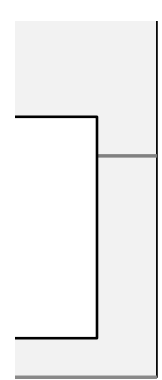

Page 161 of 203

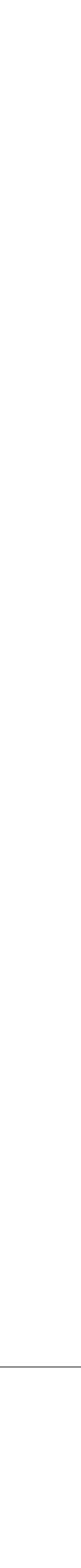


symbols are $2 \sigma$

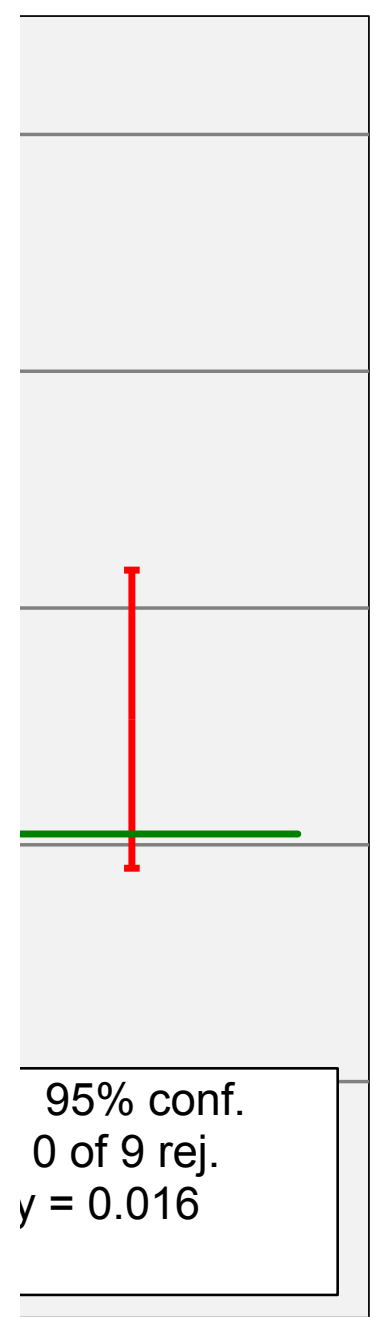


1 Pre-orogenic upper crustal softening by lower greenschist-facies metamorphic reactions

2 in granites of the central Pyrenees

3

4 Laura Airaghi ${ }^{1 *}$, Nicolas Bellahsen ${ }^{1}$, Benoît Dubacq ${ }^{1}$, David Chew ${ }^{2}$, Claudio Rosenberg ${ }^{1}$, 5 Emilie Janots ${ }^{3}$, Maxime Waldner ${ }^{1}$, Valérie Magnin ${ }^{3}$

$7 \quad{ }^{1}$ Sorbonne Université, CNRS-INSU, Institut des Sciences de la Terre de Paris, ISTeP UMR 8 7193, F-75005 Paris, France

$9 \quad 2$ Department of Geology, Trinity College Dublin, Dublin 2, Ireland

10 3 Université Grenoble Alpes, CNRS, IRD, IFSTTAR, ISTerre, 38000 Grenoble, France 11

12 *corresponding author:laura.airaghi@univ-orleans.fr, now at: Université d'Orléans, CNRS-

13 INSU, Institut des Sciences de la Terre d'Orléans, ISTO, UMR7327, Orléans, France

14

15

16

17

18

19

20

21

22

23

24

25

26 


\section{ABSTRACT}

Pre-kinematic greenschist-facies metamorphism is often observed in granites and basement units of mountains belts, but rarely dated and accounted for in orogenic cycle reconstructions. Studying pre-kinematic alteration is challenging because of its usual obliteration by subsequent syn-kinematic metamorphism often occurring at conditions typical of the brittle-ductile transition. It is however to be expected that pre-kinematic alteration has major implications for the rheology of the upper crust. In the 305 Ma-old Variscan basement of the Bielsa massif (located in the Axial Zone of the Pyrenees), successive fluid-rock interaction events are recorded in granites at temperatures below $350^{\circ} \mathrm{C}$. Combined microstructural and petrographic analysis, low-temperature thermobarometry and in situ $\mathrm{U}-\mathrm{Th} / \mathrm{Pb}$ dating of anatase, titanite and monazite show extensive pre-orogenic (pre-Alpine) and pre-kinematic alteration related to feldspar sericitization and chloritization of biotite and amphibole at temperatures of $270-350^{\circ} \mathrm{C}$ at 230-300 Ma. This event is followed by a second fluid-rock interaction stage marked by new crystallisation of phyllosilicates at temperatures of $200-280^{\circ} \mathrm{C}$ and associated with the formation of mylonitic shear zones and fractures parallel to the shear planes. $\mathrm{U}-\mathrm{Pb}$ anatase and monazite ages as well as the microtextural relationships of accessory minerals suggest an age for this event at 40-70 Ma, consistent with independent regional geology constraints. The Variscan basement was therefore softened at late- to post Variscan time, at least 150-200 Ma before the main Alpine shortening while Alpine-age compression lead to the formation of a dense net of mylonites. The associated deformation, both distributed at the scale of the Bielsa massif and localized at decametric scale in mylonitic corridors, precedes the strain localization along the major thrusts of the Axial zone. The Bielsa massif is a good example where, inherited, pre-orogenic upper crustal softening controls the deformation patterns in granitic basement units through low-grade metamorphic reactions. 
52 Key words: U-Th/Pb anatase-titanite-monazite dating, mylonites, chlorite-white mica 53 thermobarometry, Bielsa, Axial zone

\section{INTRODUCTION}

Granites deformed at the brittle-ductile transition show mineral assemblages characteristic of low-grade metamorphism. Greenschist-facies metamorphic reactions often result in the growth of phyllosilicates (e.g. white mica, chlorite) whose presence strongly affect the rheological behaviour of the upper crust through softening, strain localization and fluid migration (Tullis, Yund, \& Farver, 1996; Ingles, Lamouroux, Soula, Guerrero, \& Debat, 1999; Jackson, Austrheim, McKenzie, \& Priestley, 2004; Wintsch, Christoffersen, \& Kronenberg, 2005; Goncalves, Oliot, Marquer, \& Connolly, 2012). The breakdown of $80 \%$ of feldspar into white mica, for example, reduces the depth of the brittle-ductile transition by two thirds (Gueydan, Leroy, Jolivet, \& Agard, 2003). Reactions forming low-grade mineral assemblages responsible for crustal softening are generally observed to be syn-kinematic, occurring during the major deformation phases of an orogenic cycle (Wayne \& McCaig, et al., 1998; Gueydan et al., 2003; Mansard, Raimbourg, Augier, Précigout, \& Le Breton, 2018). Static or quasi-static hydrothermal alteration is also observed in granites and has been widely documented, especially in large igneous provinces (e.g. Parneix, Beaufort, Dudoignon, \& Meunier, 1985; Cathelineau et al., 1986; Yuguchi, Sasao, Ishibashi, \& Nishiyama, 2015). However static alteration is rarely characterised, and hardly ever dated and placed in a regional orogenic context. Indeed, lowgrade metamorphic reactions often leave tenuous fingerprints only hinted at by equivocal microtextural relationships, especially in granites (e.g. Airaghi et al., 2017b), which are generally considered poorly reactive rocks at temperatures below $400^{\circ} \mathrm{C}$.

At a microscopic scale, pre-kinematic and syn-kinematic transformations are difficult to distinguish, particularly so if the rocks have been deformed through several orogenic cycles. 
The timing and conditions of pre-kinematic metamorphic reactions and their effects on (i) the syn-kinematic transformation and (ii) the rheological response of the upper crust to successive compressive phases remain therefore poorly constrained. This study addresses these issues by focussing on the Bielsa massif, a Variscan granite located in the Axial Zone of the central Pyrenees (Figure 1a). This massif is an excellent natural laboratory to study how granitic rocks record distinct low-grade metamorphic events. The massif is part of the central Pyrenean belt, which experienced at least two well-known deformation phases (Late Variscan and Alpine/Pyrenean, Cochelin et al. 2017), which are difficult to distinguish in the Axial zone (Cochelin et al, 2017). The Bielsa massif is located in the most external basement domain of the Pyrenees (implying it experienced the lowest peak metamorphic temperatures with respect to Alpine tectonics), south of the zones of large-scale shear bands that have affected other granitic basement units of Pyrenees (e.g. Maladeta, Néouvielle; Wayne \& McCaig, 1988). In addition, structural and microstructural studies show significant distributed shortening at the scale of the massif (Román-Berdiel et al., 2004; Bellahsen et al., 2019), raising questions about the link between low-grade metamorphism and strain accommodation mechanisms. Early feldspar destabilisation has been proposed to have a major control on subsequent mechanical softening in Bielsa (Bellahsen et al., 2019), but the conditions and the age of such reaction still remains unconstrained.

In this contribution we estimate the conditions of crystallization of both pre-kinematic and synkinematic greenschist-facies mineral assemblages in the Bielsa granite and its Triassic sedimentary cover. Microstructural and petrographic analyses were combined with lowtemperature thermobarometry and in situ $\mathrm{U}-\mathrm{Th} / \mathrm{Pb}$ dating of zircon, titanite and monazite. Attempt to date anatase (polymorph of rutile) by the $\mathrm{U} / \mathrm{Pb}$ method were also undertaken. The implications for the timing of crustal softening relative to the major shortening phases are discussed with respect to their associated mineral assemblages. Results suggest that pre- 
103 kinematic (pre-Alpine) alteration controls the deformation pattern and strain localisation in the

104 Bielsa granite during Alpine shortening.

\section{THE BIELSA MASSIF IN THE AXIAL ZONE (CENTRAL PYRENEES)}

The Axial zone (central Pyrenees) is part of a S-verging thrust system made of

108 imbricated thrust units stacked during the Late Cretaceous-Early Miocene collision of the

109 Iberian and European plates (Figure 1a). The plutons of the Axial zone (i.e. Bielsa, Neuville,

110 Maladetta massifs etc., Figure 1a) were emplaced into Paleozoic sediments during Late-

111 Variscan (320-300 Ma, e.g. Paquette, Gleizes, Leblanc, \& Bouchez, 1997; Vacherat et al., 2017).

112 These plutons and their Paleozoic sedimentary cover constitute the basement to the Permian

113 and Mesozoic sedimentary units (Fig. 1a). Several tectono-metamorphic stages are documented

114 in the metasedimentary units Axial zone but are difficult to distinguish within the basement.

115 Recent studies have proposed that the majority of the deformation in the Axial zone is of

116 Variscan age (Cochelin et al., 2017). Cretaceous hydrothermal events have been identified in

117 the eastern Axial zone (Fallourd et al., 2014; Boutin et al., 2015) and related to extensive

118 tectonics centered on the SE Bay of Biscay. Basement thrusts in the central-western Pyrenees

119 (named Gavarnie and Guarga thrusts) were (re)activated during the Eocene to Miocene (20-30

120 and 32-36 Ma respectively, Jolivet et al., 2007; Elmola et al., 2018 and references therein). In

121 the hanging wall of the Gavarnie thrust, an older deformation event (c. $48 \mathrm{Ma}$ ) is recognised in

122 the massifs of Néouvielle and Eaux Chaudes. It has been associated with the activation of

123 mylonitic shear bands (e.g. Wayne \& McCaig, 1998).

124 This study focusses on the Bielsa massif, mainly constituted by a pluton emplaced at c. $305 \mathrm{Ma}$

125 (Vacherat et al., 2017), in the S-verging thrust system structurally located between the Gavarnie

126 and Guarga thrusts (Figure 1b). Close to Lake Urdiceto (Figure 1c-d), granitic to granodioritic

127 rocks (Román-Berdiel et al., 2004) are unconformably overlain by Triassic continental red 
sandstones and pelites. The basement and its sedimentary cover were deformed (folded) during the Alpine compressional phase (Figure 1d, Casas et al., 2003; Román-Berdiel et al., 2004, Bellashen et al., 2019) with a wavelength of $\sim 1 \mathrm{~km}$. Several sub-vertical S- or N-dipping normal or reverse steep faults bound the antiforms and synforms (Figure 1d). In the core of the synforms, Triassic sediments crop out. They exhibit a sub-vertical to N-dipping cleavage (e.g. Figure $2 b$ and $3 \mathrm{c}$ ), with nord side up sense of shear kinematic indicators above the sediment-cover interface (Bellahsen et al., 2019). The granitic basement is heterogeneously deformed. At the decametric scale, undeformed and weakly deformed granitic rocks alternate with zones of distributed ductile deformation, zones of fracturing and faulting and mylonites (Figures 1c-d and 2a-f). A pervasive N-dipping schistosity is widespread in the basement (Figure 2a-c and e). Mylonitic corridors, with 1-2 m wide, are observed within the basement and at its boundary with the Triassic sedimentary cover (Figure 2a-c). Mylonitic corridors alternate with less deformed rocks with a wave length of $\sim 100 \mathrm{~m}$ (Figure 1c) and they are characterized by pervasive schistosity (S) and steep, N-dipping $\left(45-70^{\circ}\right)$ and S-dipping $\left(55-70^{\circ}\right)$ shear planes (C) associated either with a north or with a south-verging movement (Figure 2a-e). In some shear zone, the dips of mylonitic planes varies from S-dipping to N-dipping, with an apparent sense of shear from top-to-north to top-to-south (Figure 2b-d). Some of the shear bands at the boundary between the Triassic sediments and the late Variscan granite (regionally trending N110, Figure 1c) contain deformed fragments of the Triassic red sandstone (Román-Berdiel et al., 2004; Figure 2b), indicating that they are post-Triassic. The high density of mylonitic corridors and the pervasive schistosity development result in a distributed deformation pattern (as observed in other crystalline units as the Aar massif, Wehrens et al., 2017). The granitic/granodioritic basement (regardless of the intensity of the strain) is pervasively hydrated and the original magmatic mineral assemblage is partly to totally replaced by greenschist-facies minerals. The steep topography of the massif allows one to infer that the latter replacement 
153 affects the pluton at least over a topographic vertical profile of $2 \mathrm{~km}$ from the lowest outcrop

154 of the massif (yellow stars in Figures 1b).

At the boundary between the Triassic sediments and the granite/granodiorite, sub-

156 horizontal quartz and chlorite-bearing veins which are 1-5 cm-wide are observed (yellow square

157 in Fig. 3b). Fifty meters from the contact, Triassic sediments show two generations of chlorite-

158 and quartz-bearing veins: (1) sub-horizontal veins parallel to the ones observed at the contact

159 between the basement and the sedimentary rocks produced by vertical extension, locally folded

160 and associated with a crenulation cleavage (N184S) and (2) sub-vertical veins (Figure 3c).

\section{SAMPLE DESCRIPTION AND CHRONOLOGY OF MINERAL ASSEMBLAGES}

Twenty-seven samples were collected along two transects east and west of Lake Urdiceto, from

164 the undeformed granite (e.g. ZA16-84 and B11-16, Figure 4a, b) up to the zone of intense 165 mylonitisation (e.g. ZA16-96, ZA16-98; Figures 1c, 4g). One sample (ZAL18-11) was

166 collected at the contact between the Triassic sandstones and a basement-hosted vein (Figures

167 3b). Two samples (ZA16-12 and ZA16-13) were collected in the Triassic sediments (Figures $1683 \mathrm{c})$.

\section{3.1 Samples from the basement}

171 In the basement unit, we distinguished the following rock fabrics: Undeformed samples (e.g.

172 ZA16-84, Figure 4a), where the magmatic, euhedral shape of grains is still preserved. Weakly deformed rocks (e.g. Figure $2 \mathrm{f}$ and $4 \mathrm{c}$ ) exhibiting a weak yet pervasive schistosity (at an outcrop scale: $\mathrm{S}$ in Figure 2a). In some of those samples the schistosity is associated to $\mathrm{mm}$-scale

176 subvertical shear planes observed at an outcrop scale in mylonites (C in Figure 2a). Mylonites

177 showing S and C planes (e.g. ZA16-133, Figure 4g), where the C planes are as pervasive as S. 
178 In the samples defined as mylonite, both brittle and ductile deformation features are identified, with the brittle ones underlined by fractures parallel to the shear planes $C$ (Figure $4 \mathrm{~g}$ ). \pm amphibole, apatite, zircon (all in textural equilibrium, Figures 4a-b) and ilmenite preserved as inclusions in the less altered amphibole and biotite grains (Figure 5a-b). The alteration is comprised by the breakdown of plagioclase and K-feldspar into white mica (10-30 $\mu \mathrm{m}$ in size) + albite + calcite \pm rare epidote (Figure 4a, c, e-f). Biotite and amphibole break down to chlorite flakes (ChlA, abbreviations are from Whitney \& Evans, 2010) \pm white mica $(20-100 \mu \mathrm{m}$ in size) \pm prehnite (Figure $4 \mathrm{a}$ ) and a Ti-bearing phase (anatase or titanite or both, Figure 4b, d, f and g). In the paragraph below, mineral assemblages are described in relation to their

189 microstructural evolution from the undeformed granites and granodiorites to mylonites. In the undeformed samples, relicts of feldspar, biotite and amphibole are still visible and their

191 euhedral magmatic grain shapes are preserved (Figure 4a). Chlorite flakes (ChlA) grow at the

192 grain boundaries of parent minerals and along biotite cleavages, and they are associated with a

193 Ti-bearing phase (see below) \pm prehnite \pm K-feldspar (Figure 4a-b). Ilmenite grains are 194 preserved in unaltered biotite and amphibole domains (Figure 5a). Quartz grains contain sparse, 195 micrometric fluid inclusions and are cross-cut by microcracks. Allanite is locally observed in 196 textural equilibrium with the magmatic minerals. Euhedral magmatic zircon grains are found 197 within relicts of feldspar or at the boundaries between feldspar and amphibole. 198 Cathodoluminescence imaging of zircon in samples ZA16-84 and ZA16-119 typically reveals 199 primary oscillatory growth zoning without internal complexity. However, some zircon grains, 200 especially in sample ZA16-119, show metamictization with porous domains and lobate rims 5$20120 \mu \mathrm{m}$ thick, and very low cathodoluminescence emission (Figure 5d). In the weakly deformed 202 samples, feldspar is pervasively sericitized (up to $80-90 \%$ ). Original biotite grains are 
203 chloritized between 70-90\% (Figure 4c). Chlorite flakes (ChlA) and secondary reaction phases 204 (quartz, Ti-phase \pm white mica) are oriented parallel to the main schistosity and tightly folded 205 to form kinks (Figure 4d). Quartz grains partly preserve their magmatic texture, but are 206 fractured, with fractures subparallel to the kink axes. Grains are cross-cut by fluid inclusion 207 trails. Originally euhedral grains of ilmenite are first altered to aggregates of anatase + calcite 208 then to titanite (Figure 5a-b), following the reaction proposed by Hansen, Reimink, \& Harlov 209 (2010) for low temperature conditions. Anatase is itself replaced by titanite, in textural 210 equilibrium with chlorite flakes (Figures 5a-b). Anatase and titanite are systematically aligned 211 with the chlorite flakes, within the S-planes. When they are folded to form kinks, anatase relicts 212 are mainly preserved in the microfold hinges (Figure 5c). In some of the weakly deformed 213 samples, titanite is the major Ti-bearing phase preserved (samples ZA16-119, B11-16, ZA1621477 and ZA16-84). In weakly deformed samples showing millimetric fractures, biotite deformation and 216 stretching along the main schistosity $\mathrm{S}$ is more pronounced (e.g. Figure 4e). Calcite is present 217 in the matrix. Fractures 1-3 mm thick displace the folded chlorite flakes to form en-echelon 218 microstructures (Figure 4e). These fractures are filled with secondary chlorite (ChlB) \pm white 219 mica (Figure 4f). Sheets of ChlB are oriented parallel to the fracture walls in contrast to ChlA grains which are mainly perpendicular (Figure 4f). Furthermore, ChlB is rarely associated with 221 biotite breakdown products as prehnite and Ti-bearing phases (Figure 4f). Quartz grains are 222 both fractured and stretched, they exhibit undulose extinction and abundant fluid inclusion trails. 223 Titanite is observed in the same microstructural position as in undeformed samples (folded 224 within ChlA flakes). The microstructural position of anatase is instead more variable. Anatase 225 is observed: (1) as $\sim 70 \mu \mathrm{m}$ sub-euhedral relicts in titanite cores at the rims of the ChlA flakes 226 (as in the undeformed samples, type-1 Ant, Figure 4h, 5b-c, f-h and Figure S1) and (2) as grains $227 \quad 20-30 \mu \mathrm{m}$ in size not associated with titanite within ChlA flakes along the ancient biotite 
cleavages (type-2 Ant, e.g. Figure 4h, 5f-h and Figure S1). Locally, type-2 anatase is observed within ChlB-bearing fractures (e.g. ZA16-73, Figure 5i). Type-2 anatase of type 2 is often observed close to type- 1 anatase. Magmatic accessory minerals such as apatite and zircon are fragmented and stretched along the S planes.

In mylonitic samples, the schistosity is defined by folded chlorite + white mica flakes and interconnected layers of white mica (Figure 4g). Flakes are folded up to kinks. Shear planes (C) form at high angle with the main schistosity $\mathrm{S}$ (Figure 4g-h). The kink axes are kinematically compatible with shear movement on the C planes. Quartz grains are partially stretched and most of them are fractured, with cracks subparallel to the $\mathrm{C}$ planes (Figure $4 \mathrm{~g}$ ). Within the grains, evidences of ductile deformation are observed such as deformation lamellae. A larger amount of fluid inclusions in quartz are observed with these samples compared to weakly deformed samples. In mylonites (ZA16-96, ZA16-98), only one type of anatase is observed (small anatase grains of type 2 predominate). In these samples, monazite grains, 10$40 \mu \mathrm{m}$ in size, were identified in mylonites (sample ZA16-98 and ZA16-88). These monazite grains are wrapped by secondary chlorite and white mica (suggesting post-magmatic origin). The largest grains of monazite $(20-40 \mu \mathrm{m})(\mathrm{Mnz} 1)$ are lobate, and stretched along the $\mathrm{C}$ planes with fractures perpendicular to them and exhibit irregular grain boundaries, micrometric large fractures, patchy textures with bright and dark spots in BSE images and inclusion-like dissolution figures' (as described by Grand'Homme et al., 2018; Airaghi, Janots, Lanari, de Sigoyer, \& Magnin, 2018, Figure 5c). Small monazite grains $(\sim 10 \mu \mathrm{m})$ with rounded shape share features with that of ZA16-88, aligned along the main foliation or within alteration halos marking the reaction front along secondary fractures subparallel to the $\mathrm{C}$ (Figure $5 \mathrm{j}$ ). In sample ZA16-98 monazite is locally Th and Si-enriched (tending towards a thorite composition, inset in Figure 5i). 
In summary, the granitic rocks experienced a first transformation of the original magmatic mineral assemblage of $\mathrm{Qz}+\mathrm{Fs}+\mathrm{Pl}+\mathrm{Bt} \pm \mathrm{Amph}(+\mathrm{Ilm}+\mathrm{Ap}+\mathrm{Zr} \pm \mathrm{Aln})$ to $\mathrm{Qz}+$ 254 ChlA + Ti-phase (Ttn or Ant then Ttn) $\pm \operatorname{Pr} \pm$ KFs. Zircon and titanite grains with anatase core (type-1 Ant) are stretched in the main schistosity and folded during shearing. They therefore predate any deformation stage. ChlB is rarely associated with secondary reaction products and helps to define the C-parallel fractures. It is considered to precipitate from a fluid during a second syn-kinematic (syn $\mathrm{C}$ planes) alteration event. The varying characteristics of anatase, only found in deformed samples, suggest that in these samples two generations of anatase may be preserved. That type- 2 anatase is located within ChlA flakes along former biotite cleavage suggests that type- 2 Ant grew after type-1 Ant on ChlA rims. The chloritization reaction front and replacement is indeed expected to progress from the grain boundaries to the core, along the biotite cleavages. The proximity of type-2 anatase to type-1 anatase grains indicates however transport scale of the anatase-forming elements limited to some dozen of $\mu \mathrm{m}$. In sample ZA1673 (discretely deformed). Type-2 Ant is observed in C-parallel fractures (Figure 5i). Type-2 Ant may have crystallized during the shearing. Mnzl is affected by deformation and mainly 267 observed within the main schistosity, while Mnz2 (and type-2 Ant, see below) are associated with $\mathrm{C}$ parallel fractures. Mnz2 is therefore syn $\mathrm{C}$ planes forming stage.

3.2 Samples from Triassic sediments and at the boundary between sediments and the 271 granodiorite At the brecciated contact between the Triassic sediments and the chlorite-quartz bearing vein in contact with the basement (Figure 6a), the Triassic sediments are composed of quartz, 274 chlorite, white mica, Fe-oxides and clasts in a calcite matrix (sample ZAL18-11). The vein is composed of mm-size quartz grains recrystallized by bulging and hosting a dense net of fluid 276 inclusions, interlayered with elongate chlorite and white mica ( \pm calcite) (Figure $6 \mathrm{~b})$. Along 
chlorite grain boundaries, abundant Fe-oxides, 20-40 $\mu \mathrm{m}$ large anatase grains, monazite, REEbearing carbonates and tourmaline are occasionally observed (Figure $6 \mathrm{~b}$ ). The textural aspect of monazite in sediments is similar to that of monazite in the basement (inset in Figure 6b).

The red-coloured, foliated pelitic rocks are comprised of white mica, chlorite and quartz grains on the scale of tents of microns ( \pm detrital monazite, apatite and anatase, samples ZAL1812 and ZAL18-13). The veins are approximately $100 \mu \mathrm{m}$ thick, they opened at high angle with the foliation and are filled by elongate grains of quartz rich in fluid inclusions (in the core of the vein). Chlorite developed along the vein-host rock interface with long axes parallel to the vein opening direction (Figure 6c). Veins are stretched and displaced by 30-50 $\mu \mathrm{m}$ secondary chlorite and quartz-bearing layers cross-cutting the foliation, forming 'en échelon' fractures (Figure 6c-d). Monazite in pelites forms euhedral grains 5-10 $\mu \mathrm{m}$ in diameter, sometimes in association with Fe-oxides and xenotime at the interface between the veins and host-rock.

\section{ANALYTICAL AND COMPUTATIONAL METHODS}

The chemical composition of metamorphic chlorite and white mica has been analysed with a CAMECA SX-FIVE electron probe micro-analyser (EPMA) at ISTeP (Sorbonne Université). Point measurements were made under a $15 \mathrm{kV}$ acceleration voltage and $10 \mathrm{nA}$ beam current with a $\sim 1 \mu \mathrm{m}$ beam size, in wavelength-dispersive spectroscopy (WDS) mode, using diopside (Ca, $\mathrm{Mg}, \mathrm{Si}), \mathrm{MnTiO}_{3}(\mathrm{Mn}, \mathrm{Ti})$, orthoclase $(\mathrm{K}, \mathrm{Al})$, hematite $(\mathrm{Fe})$, albite $(\mathrm{Na})$ and $\mathrm{Cr}_{2} \mathrm{O}_{3}(\mathrm{Cr})$ as standards for measuring the elements written in parentheses. The compositional variability of chlorite and white mica in different microstructural sites was characterised by element mapping using both the EPMA and a Zeiss SUPRA-55VP scanning electron microscope (SEM) at ISTeP (Sorbonne Université). EPMA elemental maps were acquired in WDS mode at 100 nA beam current, $15 \mathrm{keV}$ acceleration voltage with a $200 \mathrm{~ms}$ dwell time. SEM maps were acquired in energy-dispersive spectroscopy (EDS) mode at $15 \mathrm{keV}$ acceleration voltage, $30 \mathrm{nA}$ 
302 beam current with a dwell time of $256 \mu \mathrm{s}$. SEM and EPMA maps were quantified with the 303 software XMapTools (Lanari et al., 2014). The U, Th, Pb and REE contents of monazite were 304 analysed with the JEOL-JXA-8230 EPMA at ISTerre (University of Grenoble), at $15 \mathrm{keV}$ 305 acceleration voltage, $200 \mathrm{nA}$ beam current with a spot beam size. For each monazite analysis, 306 a chemical $\mathrm{U}-\mathrm{Th} / \mathrm{Pb}$ age was determined and the average age of different populations of 307 monazite (identified on the base of compositional criteria) was calculated with the software 308 NiLeDAM. The analytical protocol and data processing are detailed in Appendix 1. Titanite, anatase and zircon were dated in-situ by the U-Pb LA-ICP-MS technique at 310 Trinity College, Dublin (Ireland), on the same thin sections as used for the microstructural and 311 petrological studies. U-Pb ages were determined using a Photon Machines Analyte Exite 193 $312 \mathrm{~nm}$ ArF Excimer laser-ablation system with a Helex 2-volume ablation cell coupled to an 313 Agilent 7900 ICPMS. For titanite, zircon and anatase, 300 shots were ablated per analysis with 314 a laser spot size of $24 \mu \mathrm{m}$ (Figure S1). The laser repetition rate and fluency were set at $15 \mathrm{~Hz}$, $3151.60 \mathrm{~J} / \mathrm{cm}^{2}$ and $11 \mathrm{~Hz}$ and $2.25 \mathrm{~J} / \mathrm{cm}^{2}$ for anatase/titanite and zircon respectively, and typically 31650 analyses were obtained on each sample (usually 1-2 spots per grain) and plotted on a Tera317 Wasserburg diagram (Tera and Wasserburg, 1972) with $2 \sigma$ error ellipses, allowing 318 determination of the initial $\mathrm{Pb}$ intercept on the ${ }^{207} \mathrm{~Pb} /{ }^{206} \mathrm{~Pb}$ axis. The raw titanite and anatase isotope data were reduced using the "VizualAge 320 UcomPbine" data reduction scheme (DRS) of Chew, Petrus, and Kamber, (2014). The 321 "VizualAge" DRS was employed for zircon data reduction. Common $\mathrm{Pb}$ in the titanite and 322 rutile standards was corrected using the ${ }^{207} \mathrm{~Pb}$-based correction method. For unknowns, the 323 initial $\mathrm{Pb}$ in titanite and anatase was determined from the upper intercept (y-intercept on the $324{ }^{207} \mathrm{~Pb} /{ }^{206} \mathrm{~Pb}$ axis) of the discordia line defined by the analyses on Tera-Wasserbutg Concordia. 325 The age for each population of grains was calculated from the lower intercept of the discordia 326 line on Tera-Wasserburg Concordia. line with the $\mathrm{Pb}$ evolution model. Zircon ages were instead 
estimated by calculating the weighted average of ${ }^{206} \mathrm{~Pb} /{ }^{238} \mathrm{U}$ corrected ages (Table S3). The primary standards employed, the secondary standard ages and further details on the analytical procedure are reported in Supplementary Material S1. Titanite and anatase included in ilmenite were not dated due to their small size. When anatase core relicts were preserved in titanite grains (type-1 Ant), it was rarely possible to ablate separately relicts of anatase and their titanite host due to the small grain size (e.g. Figure S1). In anatase-dominated samples, where the bulk of grain was anatase, these analyses were classified and processed as anatase, in titanite dominated samples with only minor relict anatase the analyses were classifies as titanite. The contribution of relicts of anatase to the calculated age was estimated by comparison with results from samples where the only anatase or titanite only were sampled. In sample where two types of anatase were observed, all anatase data were processed together. The existence of one or two population(s) of anatase based on the data interpretation was compared to microstructural observations.

Monazite ages were calculated from the EPMA U, Pb and Th contents. The associated detection limits were 90 ppm for $\mathrm{Pb}, 120 \mathrm{ppm}$ for $\mathrm{U}$ and $115 \mathrm{ppm}$ for Th. For each monazite analysis, a chemical U-Th- $\mathrm{Pb}$ age was determined (see Montel et al., 1996). When the $\mathrm{Pb}$ content was lower than the EPMA detection limit, a value of 90 ppm was used to estimate a maximum age.

The crystallization temperature and the minimum content of $\mathrm{XFe}^{3+}\left(\mathrm{Fe}^{3+} /\left(\mathrm{Fe}^{2+}+\mathrm{Fe}^{3+}\right)\right)$ of each generation of chlorite in 23 selected samples were estimated using the Chl-Qz- $\mathrm{H}_{2} \mathrm{O}$ thermodynamic model of Vidal et al. (2006) from element abundances at a reference pressure of 3 kbar and $\mathrm{H}_{2} \mathrm{O}$ activity at fixed unit value. The absolute uncertainty on the crystallization temperature estimates is around $50^{\circ} \mathrm{C}$ (Vidal et al., 2006; Dubacq, Soret, Jewison and Agard, 2019), although the relative uncertainty between samples is likely lower $\left(10-20^{\circ} \mathrm{C}\right)$. The semi- 
351 empirical thermometer of Bourdelle, Parra, Beyssac, Chopin, and Vidal (2013) formulated for 352 low-temperature chlorite was used for comparison.

\section{RESULTS}

\subsection{Composition of pre-kinematic and syn-kinematic chlorite and white mica}

357 Chlorite generally exhibits compositional variation at the $\mu \mathrm{m}$-scale with high variability in Fe, $358 \mathrm{Mg}$ and $\mathrm{Al}$ contents (especially in discretely deformed granites), as shown in Figure 7 (and 359 Table S1). In samples collected east of Lake Urdiceto, two groups of chlorite are recognized. 360 The first, named Chl1, is ubiquitous from undeformed granites (pre-kinematic) to mylonites, 361 and mainly observed in ChlA flakes (Figure 7a). It should be noted that Chl1 refers to a 362 chemical composition while ChlA refers to the microtexture of chlorite (which do not necessarily correspond, e.g. Vidal et al., 2006; Airaghi et al., 2017). Chl1 is Al-poor (2.2-2.4 apfu) and Mg-rich (1.7-2.5 apfu). The second group of chlorites, Chl2, is mainly observed in syn-kinematic ChlB (Figure 7a, c-d) and exhibits higher $\mathrm{Al}$ content $(\mathrm{Al}=2.5-2.86 \mathrm{apfu})$ and

366 lower $\mathrm{Mg}$ content $(\mathrm{Mg}=1.5-1.7 \mathrm{apfu})$. The Fe content increases slightly from Chl1 (2.15-2.3 $367 \mathrm{apfu})$ to Chl2 (2.4-2.5 apfu). In undeformed samples, only homogeneous Chl1 in chlorite flakes 368 is observed (e.g. sample ZA16-84). In the most deformed samples, chlorite flakes (ChlA) 369 exhibit $\mu \mathrm{m}$-scale $\mathrm{Mg}$ and $\mathrm{Al}$ zoning and portions of Chll are pseudomorphically replaced by 370 Chl2. Chl1 is also found as relicts in ChlB in fractures, wrapped by Chl2 (Figure 7a). In samples 371 collected west of Lake Urdiceto, Chl1 in flakes is Fe-rich (1.97-2.13 apfu) and Mg-poor (2.18$3722.47 \mathrm{apfu})$ compared to $\mathrm{Chl} 2$ in veins and along fractures $(\mathrm{Fe}=1.39-1.50 \mathrm{apfu}, \mathrm{Mg}=2.83-2.95$ 373 apfu) (Figure 7b, c, e) while the Al content is typically constant (Table S1). A micron-scale 374 zoning within Chl1 flakes is observed miming cleavage planes of the original magmatic crystal 375 (biotite or amphibole) in discretely deformed samples, while Chl2 within ChlB within fractures 
is homogeneous (Figure 7b). In mylonites, chlorite shows intermediate compositions (e.g. sample ZA16-96 in Figure 7c) difficult to relate to a specific microtextural sequence due to extensive deformation. In the Triassic sediments, chlorite is Al-enriched compared to chlorite in the basement, but exhibits similar compositional trends (Figure 7c-e). Two groups are recognized in samples ZAL18-13 and ZAL18-12: one is Mg-depleted (1.7-1.97 apfu) and Alenriched (2.90-2.92 apfu) and is observed in veins, the other is Mg-enriched (2.18-2.40 apfu) or Al-depleted (2.68-2.78 apfu) and is observed within the matrix (Figure 7c-e). Only the second group of chlorite is observed in sample ZAL18-11 (Table S1).

White mica in the granitic basement shows $55-80 \%$ muscovite $(M s)$, pyrophyllite $(\mathrm{Prl})$ content in the $0-20 \%$ range and up to $\sim 30 \%$ celadonite content ( $\mathrm{Cel}$, Figure $8 \mathrm{a}$ ). White mica in fractures, veins and in the deformed matrix (syn-kinematic) exhibits lower muscovite (55-70\%) and higher (or similar) celadonite content $(20-30 \%)$ than white mica in chlorite flakes (prekinematic, $70-80 \%$ muscovite, $10-20 \%$ celadonite, Figure $8 \mathrm{a})$. The composition of white mica in sedimentary samples is similar to that of white mica from feldspar in basement samples (Figure 8b), but with lower variability. While in samples ZAL18-12 and ZAL18-11 one single group of white mica is observed, in sample ZAL18-13 two groups are identified: white mica in the veins have higher celadonite contents (18-28\%) than in the matrix (10-18\%).

\subsection{Thermobarometry of low-grade fluid-rock interaction events}

Thermodynamic modelling was combined to empirical thermobarometry to distinguish the conditions of crystallisation of the different generations of chlorite and white mica possibly recorded by the restricted range of compositional variations. Crystallisation temperature calculated with the chlorite thermometers of Vidal et al. (2006) and Bourdelle et al. (2013) are reported in Figure 9a, and range between 180 and $350^{\circ} \mathrm{C}$. The minimum content of $\mathrm{XFe}^{3+}$ in chlorite calculated with the method of Vidal et al. (2006) ranges between 0.1 and 0.55 , which 
401 is realistic for low-T chlorite (Masci et al., 2019). The entire iron content was considered 402 divalent for the thermometer of Bourdelle et al. (2013) as recommended in that study. 403 Calculated temperatures for all groups of chlorites are in the same range, with small but 404 systematic differences between Chl1 and Chl2. In samples recording two compositional groups 405 of chlorites (that correspond to pre and syn-kinematic grains), where Fe variations prevail on 406 other element variations (ZA16-82, B11-19, ZA16-78), Chl1 records higher temperatures (220$\left.407320^{\circ} \mathrm{C}\right)$ than $\mathrm{Chl} 2\left(180-250^{\circ} \mathrm{C}\right)$. In samples where dominant chemical variations involve $\mathrm{Al}$ and $408 \mathrm{Mg}(\mathrm{ZA} 16-126, \mathrm{ZA16-73,} \mathrm{B} 10-32), \mathrm{Chl1}$ records lower temperatures $\left(170-280^{\circ} \mathrm{C}\right)$ than $\mathrm{Ch} 12$ $409 \quad\left(230-350^{\circ} \mathrm{C}\right.$, Figure 8a). In other samples (ZA16-133, B10-31), where two compositional 410 groups of chlorite were observed, calculated temperature cover the entire temperature range 411 estimated for all samples. Undeformed granite samples preserving only Chl1 (e.g. ZA16-79, 412 B11-16, ZA16-117) record the highest range of temperatures (280-350 $\left.{ }^{\circ} \mathrm{C}\right)$. Crystallisation 413 temperatures calculated for chlorite in mylonites (e.g. ZA16-96, ZA16-98, ZA16-128, ZA16414 97) are intermediate between the Chl1 and Chl2 crystallisation temperatures. Two ranges of 415 temperatures were also obtained for chlorite in sedimentary samples (ZAL18-12). The highest 416 temperatures $\left(240-350^{\circ} \mathrm{C}\right)$ are generally associated with chlorite in veins, while lowest 417 temperatures $\left(180-280^{\circ} \mathrm{C}\right)$ are mostly associated with chlorites in C planes (with variability, 418 especially for samples collected Est and West of the Lake of Urdiceto, Fig. 1c). Only one range 419 of chlorite temperatures $\left(\mathrm{Chl} 2,200-300^{\circ} \mathrm{C}\right)$ is observed within the vein of sample ZAL18-11. 420 To test for the statistical validity of the two temperature intervals $\left(240-350^{\circ} \mathrm{C}\right.$ and $\left.180-280^{\circ} \mathrm{C}\right)$, 4213000 temperatures were randomly sampled from the dataset calculated with the thermometer 422 of Vidal et al. (2006) assuming a Gaussian error of $\pm 30^{\circ} \mathrm{C}$ (Figure $9 \mathrm{~b}$ ). In the basement, two main peaks at 260 and $330^{\circ} \mathrm{C}$ persist. Scattered values at low-T conditions are attributed to 424 modelling artefacts due to $\mathrm{Fe}^{3+}$ overestimation and/or to departure of some chlorite 425 compositions from equilibrium with assumed unit activity for quartz and water. 
The moderate celadonite contents of white mica (accounting for the Tschermark substitution only) suggest crystallization pressures in the range 1-4.5 kbar following the calibration of Massone and Schreyer (1988) (Figure 9c), as opposed to sub-surface hydrous alteration of feldspar only, which would favour pyrophyllitic substitution (Dubacq, Vidal, \& De Andrade, 2010). Following this calibration, the higher celadonite content (and Si apfu content) of white mica in fractures, veins and shear bands indicates higher pressure conditions than in white mica growing from feldspar or from biotite (white mica in flakes, Figure 9c).

\subsection{In situ $\mathrm{U}-\mathrm{Th} / \mathrm{Pb}$ dating of magmatic zircon and hydrothermal anatase, titanite and}

\section{monazite}

Results for titanite, anatase and monazite dating are shown in Figure 10a-c and summarized in Table 1; the raw isotopic ratios and age data for zircon, titanite and anatase are provided in Table S3. The entire geochronological dataset is combined with previous literature age constraints of metamorphic events, derived from other localities of the Axial zone in Figure 10d. The composition of monazite together with all concordias for zircon, titanite and anatase are provided in Figure S2. Zircon analyses yield weighted mean Concordia ages at 300-310 Ma (Figure 10d and f). Some zircon analyses, especially in sample ZA16-119, exhibit Pb loss and broadly define a discordia line intercepting the Concordia at c. 285-290 Ma (see Figure S1).

Titanite grains contain on average $16 \mathrm{ppm}$ of $\mathrm{U}, 1.7 \mathrm{ppm}$ of Th and $50 \mathrm{ppm}$ of common $\mathrm{Pb}$, and exhibit a $\mathrm{Th} / \mathrm{U}$ ratio of 0.1 with ages spanning from $336 \pm 12$ to $313 \pm 11$ (Figure 10a and d). Titanite in ZA16-84 provides the age constraint with the lowest uncertainty ( $313 \pm 11$, MSWD of 1.1, Figure 10a) and exhibits the lowest common Pb content (10 ppm) and the highest $\mathrm{Th} / \mathrm{U}$ ratio $(0.20)$. Anatase contains on average lower amounts of $\mathrm{U}(8 \mathrm{ppm})$ and common $\mathrm{Pb}$ (24 ppm), and higher Th contents (3.4 ppm) than titanite, with $\mathrm{Th} / \mathrm{U}$ ratios of 0.4 , with ages from 301 to $48 \mathrm{Ma}$ (Figure 10b and d). The age of anatase is difficult to constrain because of 
451 high common $\mathrm{Pb}$ and low radiogenic $\mathrm{Pb}$ contents. When possible, two Tera-Wasserburg 452 concordia lower intercepts for anatase $\mathrm{U}-\mathrm{Pb}$ age data were defined for each sample, delimiting 453 the possible youngest and oldest grain populations (e.g. Figure 10b). In this case, youngest ages 454 cluster at c. 30-80 Ma while oldest ages cluster at c. 240-280 Ma (Figure 10b, d). The mylonitic 455 granite ZA16-96 exhibits the young age population only (Tera-Wasserburg concordia lower 456 intercept of $39 \pm 8 \mathrm{Ma}$, Figures $10 \mathrm{c}$ and $\mathrm{S} 1$ ), despite the low variability of the radiogenic $\mathrm{U} / \mathrm{Pb}$ 457 ratio.

Patchy monazite in samples ZA16-98 and ZAL18-11 shows low and variable U and Th contents (Figure 10e and Table S2), typical of low-temperature secondary monazite 460 (Rasmussen \& Muhling, 2007; Janots, Berger, \& Engi, 2011). The U-Th/Pb ages (with $\mathrm{Pb}>$ 461 EPMA detection limits of $\sim 100 \mathrm{ppm}$ ) range between 180 and $325 \mathrm{Ma}$ (Figure 10c). In both 462 samples, $\mathrm{Pb}$-depleted domains $(\mathrm{Pb}<$ EPMA detection limits despite Th contents up to few wt $\%$, 463 Table S2) are observed within Th-depleted monazite grains, indicating late partial replacement 464 of monazite grains by recrystallization and dissolution-reprecipitation (Figure 10e). The oldest 465 ages generally correspond to monazite with relatively high $\mathrm{Th}$ content $\left(\mathrm{ThO}_{2}>2.4 \mathrm{wt} \%\right)$ and 466 variable $\mathrm{U}$ content $\left(0.04 \mathrm{wt} \%<\mathrm{UO}_{2}<1.0 \mathrm{wt} \%\right)$. They cluster around $254 \pm 6 \mathrm{Ma}$. A second 467 group of monazite has a lower Th content $\left(\mathrm{ThO}_{2}<1.9 \mathrm{wt} \%\right)$, variable $\mathrm{U}$ content $(0.01 \mathrm{wt} \%<$ $468 \mathrm{UO}_{2}<1.9 \mathrm{wt} \%$ ) and provides an age of $196 \pm 18$ Ma. In both samples ZA16-98 and ZAL18-11 469 young ages are estimated from the $\mathrm{Pb}$-depleted domains $(\mathrm{Pb}<$ detection limits). Assuming a $\mathrm{Pb}$ 470 content equal to the EPMA detection limit ( $\sim 90 \mathrm{ppm})$, a maximum age for this late stage 471 crystallisation has been calculated at c. $100 \mathrm{Ma}$ in sample ZA16-98 and at c. 20 Ma in sample 472 ZAL18-11 for the domains with the highest $\mathrm{Th}\left(\mathrm{ThO}_{2}=7.6\right.$ wt.\% $)$ and $\mathrm{U}$ contents $\left(\mathrm{UO}_{2}=0.9750\right.$ 473 wt.\%) (Figure 10c). Cenozoic ages of c. 50 Ma have also been estimated in sample ZA16-98 474 from thorite-monazite domains. No ages could be obtained for monazite from the sedimentary 475 samples due to their low $\mathrm{U}$, Th and $\mathrm{Pb}$ contents. 


\section{DISCUSSION}

478

\subsection{Evaluation of geochronological results}

480

481

482

483

484

Estimated crystallisation temperatures are far below the closure temperature of $\mathrm{Pb}$ diffusion in rutile $\left(580-630^{\circ} \mathrm{C}\right)$, titanite $\left(575-700^{\circ} \mathrm{C}\right)$, monazite $\left(\sim 800^{\circ} \mathrm{C}\right)$ and zircon $\left(\sim 900{ }^{\circ} \mathrm{C}\right.$, Rubatto, 2017; Engi, 2017 and references therein). Therefore, $\mathrm{U}-\mathrm{Th} / \mathrm{Pb}$ dates are interpreted as crystallisation ages. Zircon is dominantly of magmatic origin as attested by its microtextures. Concordant zircon ages, inferred to bracket the timing of the Bielsa pluton emplacement at 300$310 \mathrm{Ma}$ (Figure 10d and Table 1), are in agreement with previously published ages (Vacherat et al., 2017). Zircon ages therefore constrain the upper limit of the first alteration event. Discordant ages defining a chord with an intercept at c. 290 Ma in sample ZA16-119 (Figure S1) derive from sampling of metamictic zircon domains, characterized by decreasing crystallinity, $\mathrm{Pb}$ loss and variable incorporation of common $\mathrm{Pb}$. Since metamict domains show typical textures of dissolution-reprecipitation under low-T conditions (Geisler et al., 2003) and are observed in undeformed samples, the c. 290 Ma age may indicate re-opening of the system during the early pre-kinematic fluid-rock interaction, started shortly after the pluton emplacement, in Late Variscan times.

Titanite ages are less accurate but cluster within uncertainty of the zircon ages, at c. 300 Ma (Late Variscan) (Figure 10d and Table 1). Some of the titanite ages are older than the corresponding zircon ages from the same sample. The titanite Discordia line on TeraWasserbourg Concordia diagram is well constrained due to the large variation in $\mathrm{U}: \mathrm{Pb}$ ratio of the titanite grains (Figure 10a and Figure S2). Therefore, the titanite ages marginally older than their corresponding zircon ages may originate from local incorporation of radiogenic PB at the time of titanite crystallization (see also Essex and Gromet, 2000). The ${ }^{207} \mathrm{~Pb} /{ }^{206} \mathrm{~Pb}$ initial ratio of metamorphic titanite has been shown to vary up to $2 \%$ in the same sample (Kohn, 2017), and 
502 is often significantly lower (i.e. more radiogenic) than the crustal evolution model of Stacey 503 and Kramers (1975). This signs the incorporation of radiogenic Pb from the breakdown of a U504 rich mineral phase such as rutile or anatase, anatase being observed here as the most common 505 titanite precursor.

The $\mathrm{U} / \mathrm{Pb}$ ages obtained for anatase are less well constrained than the titanite due to 507 higher common $\mathrm{Pb}$, lower radiogenic $\mathrm{Pb}$ contents and the smaller spread in $\mathrm{U}-\mathrm{Pb}$ ratio of the 508 anatase grains. Calculated ages range between $40 \mathrm{Ma}$ and $280 \mathrm{Ma}$. Anatase does not record the 509 magmatic stage since it is observed to replace magmatic ilmenite. If type-1 Ant or titanite recrystallize into type- 2 Ant, inter and intra-grain variations in the radiogenic $\mathrm{Pb}$ and $\mathrm{U}$ content 511 could be inherited from the parent mineral given the small transport scales for anatase-forming 512 elements deduced from microtextural evidence (see section 3.1). Such variations and possible 513 incorporation of type-1 Ant in spot ablations of titanite (see section 4) contribute to the large 514 spread of the anatase ages (e.g. samples ZA16-79, ZA16-73). However, a recurrently young signal of c. 40-80 Ma is exclusively observed only in samples with both types of anatase and in 516 mylonites. As solid-state $\mathrm{Pb}$ diffusion can be excluded due to the low metamorphic grade, the 517 youngest $\mathrm{U}-\mathrm{Pb}$ anatase ages likely indicate partial (re-)crystallization of the smallest anatase 518 grains (type-2 Ant), in agreement with microtextural observations. In addition, the upper limit 519 of the range of anatase ages is the youngest in samples with both types of anatase: This suggests 520 mixing at a smaller scale than laser beam size between older titanite-anatase and new anatase. 521 The two age end-members are shown on Figure 10d for all anatase-bearing samples.

522 Monazite is unlikely to record the magmatic stage since (1) magmatic allanite and apatite 523 incorporate the rare earth elements and phosphorous necessary for monazite crystallization, (2) 524 monazite is observed to replace magmatic allanite or apatite and (3) the oldest monazite ages 525 are younger than the zircon ages. Monazite ages in both samples ZA16-98 and ZAL18-11 526 indicate a series of events at c. $250 \mathrm{Ma}, 200 \mathrm{Ma}$, and possibly $<50 \mathrm{Ma}$. This range of ages 
suggests successive episodes of partial dissolution-reprecipitation, as also shown by the patchy textures and the dissolution figures of monazite grains (Figures $5 \mathrm{c}$ and $6 \mathrm{~b}$ ). The mineral assemblage corresponding to the monazite ages of $c$. 200 Ma remains difficult to identify as textural and chemical evidences is lacking. The ages of 190-250 Ma obtained in monazite of chlorite-bearing veins in sediments indicate that monazite grains were likely inherited from the underlying basement and partially re-crystallized during vein opening and fluid circulation soon after sediments deposition in Triassic times.

\subsection{Timing and conditions of fluid-rock interactions and deformation}

Detailed microstructural and petrological observations show a poly-phase alteration history in the Bielsa basement at temperatures below $350^{\circ} \mathrm{C}$. Within this temperature range, a first fluidrock interaction event is related to the breakdown of the original magmatic assemblage and the growth of Ttn, Chl1, anatase (type- 1 Ant) at $270-350^{\circ} \mathrm{C}$. This alteration event predates all deformation stages since the earliest deformation phase deforms this mineral assemblage and the alteration event is also observed in undeformed samples (e.g. Figure 4a-b). During the first alteration event, the source of $\mathrm{Al}$ and $\mathrm{Ca}$ for chlorite and titanite ( \pm prehnite) growth is the contemporaneous breakdown of plagioclase and the albitization of alkali-feldspar (always largely consumed), irrespective of the strain intensity. Calcium may also derive from external fluids as attested by calcite growth within weakely deformed granite matrix. Titanite, zircon, anatase and oldest monazite ages allow bracketing the timing of the early alteration(s) to 230$300 \mathrm{Ma}$ (late to post-Variscan). This age range is in agreement with ${ }^{40} \mathrm{Ar} /{ }^{39} \mathrm{Ar}$ ages of biotite of c. 280 and 250 Ma obtained in the Bielsa and Maladeta massifs respectively (Jolivet et al., 2007).

The second fluid-rock interaction event is related to the syn-kinematic growth of Chl2 during the formation of C-S structures (see section 3 ) at $\sim 200-280^{\circ} \mathrm{C}$. The pervasive schistosity, kinking, shearing and fracturing are likely to have occurred at the same time since they are 
552 kinematically compatible. The S planes are observed in both the basement and in the Triassic

553 sediments (see section 2). The S-forming stage is therefore post-late Triassic in age at least.

In such deformed samples, the youngest ages are yielded by anatase (in samples bearing

both types of anatase) and by monazite; this suggests that deformation triggered the partial recrystallization of anatase and monazite. The youngest anatase ages only (c.40 Ma) and the youngest monazite ages (c. $50 \mathrm{Ma}$ ) are obtained in mylonites (ZA16-96 and ZA16-98). This observation suggests that the mylonitization related to the deformation stage likely occurred between 40 and $70 \mathrm{Ma}$. These ages are in agreement with $\mathrm{Rb}$-Sr ages of $c$. $48 \mathrm{Ma}$ obtained for the (re)activation of mylonitic bands in the Néouvielle massif $(\sim 15 \mathrm{~km}$ to the northwest of

561 Bielsa, Wayne \& McCaig, 1998). In sample ZA16-79 an age population at c. $120 \mathrm{Ma}$ is

562 observed, but no clear textural relationships are identified, while the large uncertainties mean it Eocene ages.

Chlorite temperatures calculated with the method of Vidal et al. (2006) and Bourdelle et al. (2013) are consistent despite differences in how they employ the oxidation state of Fe.

566 The calculated range of temperatures $\left(200-350^{\circ} \mathrm{C}\right)$ is also consistent with hydrothermal conditions typically associated with the appearance of prehnite and titanite in the cleavages of

568 biotite (e.g. Eggleton \& Banfield, 1985; Morad, Sirat, El-Ghali, \& Mansurbeg, 2011; Yuguchi 569 et al., 2015) and with temperatures calculated for the overlying sediments, suggesting that fluids 570 were freely circulating between the basement and the overlying sediments. In details, 571 thermometric results indicate that the pre-kinematic greenschist-facies metamorphism occurred 572 at higher temperature $\left(270-350^{\circ} \mathrm{C}\right)$ than later syn-kinematic fluid-rock interaction $\left(200-280^{\circ} \mathrm{C}\right.$, 573 Figure 9b), with spatial variations (east and west of Lake Urdiceto, Figure 9a) due to local 574 variations in fluid composition. The systematic differences between temperatures estimated for 575 Chl1 and Chl2 support however the existence of two different fluid-rock interaction events. 
The moderate celadonite content of white mica, especially in fractures, veins and shear bands (Figure 8a and c) suggests crystallization at a pressure of 1-5 kbar (1 - $4.5 \mathrm{kbar}$, following the formulation of Massone and Schreyer, 1988, Figure 9c and below 3 kbar according to Cathelineau et al., 1986 for hydrothermal alteration in granites). No paragonite nor margarite components are observed within our white mica dataset. Pyrophyllite substitution, which increases the $\mathrm{Si}$ content of muscovite, is not predominant compared to the Tschermak substitution (Figure 8a). Increasing pyrophyllite content in sample B11-31 implies crystallization during continuous cooling (Dubacq et al., 2010). Fluid inclusion trapping pressures in the range 2-5 kbar are reported by Henderson and McCaig (1996), consistent with pressure estimates from white mica in mylonite veins (Wayne \& McCaig, 1998). The lowest pressure values of $\sim 2 \mathrm{kbar}$ are consistent with the maximum burial depth estimated for the Bielsa (Jolivet et al., 2007; Bellahsen et al., 2019).

This low-grade petro-chronological dataset allows the timing of low-grade metamorphism and deformation to be discussed in the light of the Variscan and Alpine orogenic cycles, with major implications for the crustal rheology and the evolution of the deformation style in granites.

\subsection{Pre-orogenic alteration and implications for crustal softening prior to shortening}

Petro-chronological results and microstructural analyses show that Late to post-Variscan lower greenschist-facies metamorphism in the Bielsa massif transformed an initially strong assemblage made of plagioclase, K-feldspar (forming together close to half of the primary modal composition) and amphibole into weaker, hydrated minerals such as white mica and chlorite, without associated deformation (as suggested by Bellashen et al., 2019). The breakdown of feldspar to white mica decreases by up to two orders-of-magnitude the sustained shear stress (at a constant strain rate) at $400^{\circ} \mathrm{C}$, lowering the depth of the brittle-ductile 
601 transition by at least a third (Wintsch et al., 1995; Gueydan et al., 2003). In the Bielsa massif, 602 pre-orogenic sub to lower greenschist-facies metamorphic reactions have two effects: they 603 enhance grain size reduction (e.g. from magmatic feldspars to minute white micas) and also 604 replace strong minerals such as feldspar and amphibole with mechanically weaker 605 phyllosilicates (e.g. Shea \& Kronenberg, 1993). Both processes are known to result in enhanced 606 softening of the granitic rocks (e.g. Fitz Gerald \& Stünitz, 1993; Wintsch et al., 1995; Oliot, 607 Goncalves, Marquer, 2010). Early pre-kinematic alteration therefore considerably softened the 608 granite at least $150 \mathrm{Ma}$ before the major Alpine shortening phases. Furthermore, the massive 609 growth of pre-kinematic and pre-Alpine white mica and chlorite indicates high fluid-rock ratios 610 during Late-to post-Variscan times and may strongly influence the water availability in the 611 rocks at the grain boundaries during deformation. This has been shown to have major effects 612 on the mechanisms of strain accommodation in other major phases as quartz (e.g. Palazzin et 613 al., 2018) and hence impact the deformation style. Sericitisation of feldspar and chloritisation of biotite is observed to increase with 615 deformation. This suggests that granite alteration continued during syn-kinematic Alpine 616 shortening. In mylonites, syn-kinematic chlorite and white mica partly replace the pre617 kinematic chlorite and mica grains, this time without needing huge supplementary amounts of 618 external fluid. Consequently, in the latter fluid-rock interaction event, there is no evidence for 619 additional large-scale reaction-driven softening outside mylonitic zones, consistent with 620 observations reported for the Néouvielle massif ( $15 \mathrm{~km}$ to the northwest, Wayne \& McCaig, 621 1998). The crystallisation of new phyllosilicates and the larger amount of fluid inclusions in quartz in fractured and mylonitic samples as well as the partial age resetting up to 40 Ma close to shear zones also appear to indicate progressive localisation of metamorphism with strain 624 localisation. 
Zones of fractures and faults indicate that granitic rocks were also deformed by frictional behaviour, as commonly observed at temperatures of $200-350^{\circ} \mathrm{C}$ (e.g. Copley, 2017). The crystallization of phyllosilicates during a pre-orogenic alteration event may weaken fault zones by at least $30 \%$ as phyllosilicates have much lower coefficients of friction than undeformed and unaltered crystalline rocks (e.g. Kaduri, Gratier, Renard, Çakir, \& Lasserre, C., 2017; Copley, 2017). The pre-existence of phyllosilicate in the Bielsa basement therefore appears to have generated a network of weak rocks with low friction coefficient resulting in their distributed deformation.

\subsection{Effect of the early alteration on deformation patterns at field scale}

The pervasive schistosity (S) observed within the Bielsa massif along the studied transects indicates that the deformation is distributed at the scale of the massif (Figure 1b). The existence of mylonitic corridors shows a phase of strain localization with variations in the sense of shear at the kilometric to decametric scale, but their high density also results in a distributed deformation at the massif scale. At Bielsa, both $\mathrm{S}$ and $\mathrm{C}$ forming stages post-date the Late to post-Variscan pervasive alteration that softened the basement. In the Maladeta massif, (50-60 $\mathrm{km}$ to the northeast), less pervasive pre-kinematic alteration is observed as well as localized deformation and lower density of mylonitic corridors (Leblanc, Gleizes, Lespinasse, Olivier, \& Bouchez, 1994). This indicates that pre-orogenic crustal softening controlled the deformation patterns observed on a outcrop scale, in particular the frequency of mylonitic bands the location of faults and of fractures.

In the Bielsa massif, strain localization in mylonitic bands may have occurred during 647 the intense folding of the basement and the sedimentary cover in the Eocene as indicated by the 648 young ages from the mylonites. During this stage, the re-activation of steep faults may have served as fluid pathways and triggered alteration. Interfaces between differently-altered 
650 domains formed different phase strength contrasts. Consequently, ductile strain and reactions

651 interacted positively to produce narrow ductile shear zones (as described by Holyoke \& Tullis,

652 2006). Furthermore, deformation at this stage may strongly influence the breakdown on residual

653 feldspar to muscovite (e.g. Knipe \& Wintsch, 1985), as attested by the greater amount of white

654 mica observed in mylonites compared to weakly and undeformed samples.

655 The youngest ages obtained in this study (c. $40 \mathrm{Ma}$ ) are older than the activation of the

656 Gavarnie and Guarga thrusts (Figure 1b), in the hanging and footwall of the Bielsa unit, dated 657 at 32-36 Ma et 20-30 Ma respectively (e.g. Bosch et al., 2016; Elmola et al., 2018) and similar 658 to the age of mylonitization in the Néouvielle massif (Wayne \& McCaig, 1998). This may 659 indicate that the distributed shortening in Bielsa and at a larger scale predates the localization 660 of the strain along some of the major thrust of the Axial zone. These findings are consistent 661 with the distributed / localized modes of shortening documented in the external Western Alps 662 (Bellahsen et al., 2014), in the central Alps (Wehrens, Baumberger, Berger \& Herweg, 2017) 663 and in the Longmen Shan where pre-kinematic and a syn-kinematic greenschist-facies 664 metamorphic events were also recognized (Airaghi et al., 2017b), indicating that they might be 665 typical of the evolution of basement massif in the external domains of the orogenic wedges.

\subsection{Onset and preservation of early alteration in granitic basement}

668 Late to post-Variscan fluid-rock interaction took place on the scale of the Axial zone as 669 metamorphic reactions similar to those observed in the Bielsa massif are found in other crystalline massifs in the Pyrenees (e.g. Maladeta, Néouvielle, Col de Jau; Cathelineau, 1986;

671 Boutin et al., 2016). The early alteration stages may be linked to protracted magmatic activity

672 recorded in the Pyrenean domain throughout the entire Permian (Denèle, Paquette, Olivier, \& 673 Barbey, 2012). Late Variscan magmatic activity has also been invoked as the cause of 674 hydrothermal alteration in the French Massif Central (Cathelineau, 1986; Turpin, Leroy, \& 675 Sheppard, 1990). Permian to Triassic ages similar to those obtained for fluid-rock interaction 
events in the Bielsa massif have also been documented in the Variscan basement of the Eastern Iberian Central System and in NE Spain by fluid inclusion and isotopic studies (Tornos, Delgado, Casquet, \& Galindo, 2000 and references therein). The associated hydrothermal alteration in these cases is related to hydrothermal cells formed during extensional tectonic events rather than to plutonism (Tornos et al., 2000; Cardellach, Canals, \& Grandia, 2002). In the Bielsa massif, high fluid-rock ratios have been proposed to result from ingress of seawater and meteoric fluids during the extensional phase following the Variscan orogeny (McCaig, Tritlla, \& Banks, 2000). This is consistent with the fact that the Bielsa basement was exposed at the surface before or during the Permian, as attested by the erosional unconformity between the basement and its sedimentary cover. The preservation of early alteration features in granites over two orogenic cycles (Variscan and Alpine) implies that the basement remained at low grade conditions for over 200 Ma. In the Pyrenees, the main post-Variscan metamorphic phase related to fluid circulation is dated at $110 \mathrm{Ma}$ (e.g. Boutin, 2016). Although Cretaceous ages were obtained for samples which contain both types of anatase, the large $\mathrm{U} / \mathrm{Pb}$ age uncertainties for this time period in our petro-chronological dataset mean that strong evidence for extensive fluid circulation, mineral replacement and deformation during Cretaceous times is lacking. This is in contrast to the eastern Pyrenees where a hydrothermal event characterized by newly formed rutile and titanite associated with Cretaceous rifting overprints the 'post'-Variscan hydrothermal alteration assemblages (Figure 10d; Boutin et al., 2016; Fallourd et al., 2014). This may indicate that, within the Pyrenean belt, metamorphism related to rifting has only weakly affected the southern part of the Axial zone.

\section{CONCLUSIONS}

The granitic basement rocks in the southern Axial zone of the Pyrenees (Bielsa massif) acted as reactive rocks at low temperatures $\left(\mathrm{T}<350^{\circ} \mathrm{C}\right)$ over a long $(c .300 \mathrm{Ma})$ polyphase history. 
701 At an outcrop scale, the Bielsa pluton and the Triassic sedimentary cover exhibit a pervasive

702 N-dipping schistosity, folded or associated to steep C planes localized within a dense network 703 of mylonitic bands. Within the granitic basement, partial mineral replacement and 704 compositional variations at the micron-scale record an extensive fluid-rock interaction event at 705 $\sim 270-350^{\circ} \mathrm{C}$ in Late to post-Variscan times (230-300 Ma), as indicated by the titanite, anatase and zircon $\mathrm{U} / \mathrm{Pb}$ ages dataset. This event is ubiquitous and particularly well preserved in the 707 less deformed areas of the massif. It is responsible for pre-kinematic (pre-Alpine) plagioclase 708 and amphibole replacement with growth of phyllosilicates, some tens of million years after

709 pluton emplacement. A second syn-kinematic fluid-rock interaction event is marked by the 710 (re)crystallisation of phyllosilicates at $\sim 200-280^{\circ} \mathrm{C}$ and is related to the formation of mylonitic 711 corridors and fractures parallel to the $\mathrm{C}$ shear planes. In the most deformed samples, despite the 712 low radiogenic $\mathrm{Pb}$ content, monazite and anatase $\mathrm{U}-\mathrm{Th} / \mathrm{Pb}$ ages systematically show a young 713 age component at 40-70 Ma, coeval with the Alpine/ Pyrenean deformation in the southern 714 Axial zone and related to the second alteration event. As the strength of phyllosilicate-rich 715 material is lower by up to an order-of-magnitude than that of the original granite, the granitic 716 massif was significantly softened (and hydrated) at least 150-200 Ma before the major Alpine 717 shortening phase. The dense network of mylonites in the Bielsa pluton resulted in distributed 718 deformation at the massif scale before the localization of the strain along the major thrusting 719 systems of the Axial zone. Pre-orogenic crustal softening controlled the deformation patterns 720 where strain and shortening localized during successive deformation events. Pre-orogenic 721 greenschist-facies metamorphism is often poorly characterized compared to higher-grade 722 metamorphic domains in mountain belts. The case of the Bielsa massif shows that moderate 723 analytical effort can provide valuable information allowing for a better constraints on the 724 rheological and thermobarometric evolution of basement rocks during the orogenic cycle, even 725 at low-grade conditions. 


\section{ACKNOWLEDGEMENTS}

This study was founded by the CNRS-BRGM-TOTAL 'OROGEN' project. We acknowledge

Prof. S. Duchene and the anonymous review for their constructive comments and remarks.

We thank Michel Fialin, Nicolas Rividi and Omar Boudouma for their help with the electron

731 microscopy at ISTeP. DC acknowledges support from Science Foundation Ireland grants

732 12/IP/1663, 15/IA/3024 and 13/RC/2092 (iCRAG Research Centre). iCRAG is funded under

733 the SFI Research Centres Programme and is co-funded under the European Regional

734 Development Fund. We also thank Gary O’Sullivan (Trinity College) for his support with the LA-ICP-MS analysis. Authors have no conflict of interest to declare.

\section{REFERENCES}

Airaghi, L., Janots, E., Lanari, P., de Sigoyer, J. \& Magnin, V. (2018). Allanite petrochronology in fresh and retrogressed garnet-biotite metapelites from the Longmen Shan (eastern Tibet), Journal of Petrology, in press.

Airaghi, L., de Sigoyer, J., Lanari, P., Guillot, S., Vidal, O., Monié, P., ... Tan, Xibin (2017). Total exhumation across the Beichuan fault in the Longmen Shan (eastern Tibetan plateau, China) : Constraints from petrology and thermobarometry. Journal of Asian Earth Sciences, 140, 108-121.

Bellahsen, N., Mouthereau, F., Boutoux, A., Bellanger, M., Lacombe, O., Jolivet, L., ... Rolland, Y. (2014). Collision kinematics in the western external Alps, Tectonics, 33.

Bellahsen, N., Bayet, L., Denele, Y., Waldner, M., Airaghi, L., Rosenberg, C. .. Vacherat, A. mylonties and crustal strength. Tectonophysics, 766, 433-452. 
750 Bosch, G. V., Teixell, A., Jolivet, M., Labaume, P., Stockli, D., Domènech, M., ...Monié, P. (2016). Timing of Eocene-Miocene thrust activity in the Western Axial Zone and Chaînons Bérnais (west-central Pyrenees) revealed by multi-method thermochronology. Comptes Rendus Geoscience, 348(3-4), 246-256.

Bourdelle, F., Parra, T., Beyssac, O., Chopin, C. \& Vidal, O. (2013). Clay minerals as geothermometer: A comparative study based on high spatial resolution analyses of illite and chlorite in Gulf Coast sandstone (Texas, U. S.A.). American Mineralogist, 98, 914926.

Boutin, A., de Saint Blanquat, M., Poujol, M., Boulvais, P., de Parseval, P., Rouleau, C., ...Robert, J.-F. (2016). Succession of Permian and Mesozoic metasomatic events in the eastern Pyrenees with emphasis on the Trimouns talc-chlorite deposit. International Journal of Earth Science, 105, 747-770.

Cardellach, E., Canals, A. \& Grandia, F. (2002). Recurrent hydrothermal activity induced by successive extensional episodes: the case of the Berta F-(Pb-Zn) vein system (NE Spain). Ore Geology Reviews, 22, 133-141.

Casas, A.M., Oliva, B., Román-Berdiel, T. and Pueyo, E. (2003). Basement deformation: tertiary folding and fracturing of the Variscan Bielsa granite (Axial zone, central Pyrenees). Geodinamica Acta, 16(2-6), 99-117.

Cathelineau, M. (1986). The Hydrothermal Alkali Metasomatism Effects on Granitic Rocks : Quartz Dissolution and Related Subsolidus Changes. Journal of Petrology, 27, 945-965.

Chew, D. M ; Petrus, J. A. \& Kamber, B. S. (2014). U-Pb LA-ICPMS dating using accessory mineral standards with variable common Pb. Chemical Geology, 363, 185-199. 
tectonometamorphic evolution of the Axial Zone. Journal of the Geological Society, 175(2), 336.

776

777
Copley, A. (2017). The strength of earthquake-generating faults. Journal of the Geological Society, 10.1144/jgs2017-037.

Denèle, Y., Paquette, J.-L., Olivier, P. \& Barbey, P. (2012). Permian granites in the Pyrenees: the Aya pluton (Basque Country). Terra Nova, 24, 105-113.

Dubacq, B., Soret, M., Jewison, E., Agard, P. (2019). Early subduction dynamics recorded by the metamorphic sole of the Mt Albert ophiolitic complex (Gaspé, Quebec). Lithos, 334 - 335, 161 - 179, doi: 10.1016/j.lithos.2019.03.019.

Dubacq, B., Vidal, O., De Andrade, V., 2010. Dehydration of dioctahedral aluminous phyllosilicates: thermodynamic modelling and implications for thermobarometric estimates. Contributions to Mineralogy and Petrology 159, 159-174.

Eggleton, R. A. \& Banfield, J., F. (1985). The alteration of granitic biotite to chlorite. American Mineralogist, 70, 902-910.

Elmola, A. A., Buatier, M., Monié, P., Labaume, P., Trap, P., \& Charpentier, D. (2018). 40Ar/39Ar muscovite dating of thrust activity: a case study from the Axial Zone of the Pyrenees. Tectonophysics, in press.

Engi, M. (2017). Petrochronology Based on REE-Minerals: Monazite, Allanite, Xenotime, Apatite. In: Petrochronology: Methods and Applications, Kohn, M. J., Engi, M. \& Lanari, P. Reviews in Mineralogy \& Geochemistry.

Fallourd, S., Poujol, M., Boulvais, P., Paquette, J.-L., de Saint Blanquant, M., \& Rémy, P. (2014). In situ LA-ICP-MS U-Pb titanite dating of Na-Ca metasomatism in orogenic belts: the North Pyrenean example. International Journal of Earth Science, 103, 667682. 
798 Fitz Gerald, J. D. \& Stünitz, H. (1993). Deformation of granitoids at low metamorphic grade. I: Reaction and grain size reduction. Tectonophysics, 221 (3-4), 269-297.

800 Geisler, T., Rashwan, A. A., Rahn, M. K., Poller, U., Zwingmann, H., Pidgeon, R. 801 T., ...Tomaschek, F. (2003). Low-temperature hydrothermal alteration of natural 802 803 metamict zircon from the Eastern Desert, Egypt. Mineralogical Megazine, 67(3), 485508.

\section{4}

805

806

807

808

809

810

Goncalves, P., Oliot, E., Marquer, D. \& Connolly, J. A. D. (2012). Role of chemical processes on shear zone formation: an example from the Grimsel metagranodiorite (Aar massif, Central Alps). Journal of Metamorphic Geology, 30(7), 703-722.

Grand'Homme, A., Janots, E., Seydoux-Guillaume, A.M., Guillaume, D., Magnin, V., Hövelmann, J., ...Boiron, M.C. (2018) Mass transport and fractionation during monazite alteration by anisotropic replacement. Chemical Geology, 484, 51-68.

Gueydan, F., Leroy, Y.M., Jolivet, L., \& Agard, P. (2003). Analysis of continental midcrustal strain localization induced by microfracturing and reaction-softening. Journal of Geophysical Research, 108(B2), 2064.

Henderson, I. H. C. \& McCaig, A.M. (1996). Fluid pressure and salinity variations in shear zone-related veins, central Pyrenees, France: Implications for the fault-valve model. Tectonophysics, 262, 321-348.

Hansen, E., Reimink, J. \& Harlov, D. (2010). Titaniferous accessory minerals in very low-grade metamorphic rocks, Keweenaw Peninsula Michigan, USA. Lithos, 116, 167-174.

Ingles, J., Lamouroux, C., Soula, J.-C., Guerrero, N. \& Debat, P. (1999). Nucleation of ductile shear zone in a granodiorite under greenschist facies conditions, Néouvielle massif, Pyrenees, France. Journal of Structural Geology, 21, 555-576.

Jackson, J. A., Austrheim, H., McKenzie, D. \& Priestley, K. (2004). Metastability, mechanical strength, and support of mountain belts. Geology, 32(7), 625-628. 
Janots, E., Berger, A. \& Engi, M. (2011). Physico-chemical control on the REE minerals in chloritoid-grade metasediments from a single outcrop (Central Alps, Switzerland). Lithos, 121, 1-11.

Jolivet, M., Labaume, P., Monié, P., Brunel, M., Arnaud, N., \& Campani, M. (2007). Thermochronology constraints for the propagation sequence of the south Pyrenean basement thrust system (France-Spain). Tectonics, 26, TC5007.

Kaduri, M., Gratier, J.-P., Renard, F., Çakir, Z., \& Lasserre, C. (2017). The implications of fault zone transformation on aseismic creep: Example of the North Anatolian Fault, Turkey, Journal of Geophysical Research : Solid Earth, 122, 4208-4236.

Knipe, R. J. \& Wintsch, R. P. (1985). Heterogeneous, deformation, foliation development, and metamorphic processes in a polyphaser mylonite. In: Thompson, A. B., Rubie, D. C. (Eds.), Advances in Physical Chemistry, 4, Springer-Verlag, New York, 180-210.

Kohn, M. J. (2017). Titanite petrochronology, In: Petrochronology: Methods and Applications, Kohn, M. J., Engi, M. \& Lanari, P. Reviews in Mineralogy \& Geochemistry.

Lanari, P., Vidal, O., De Andrade, V., Dubacq, B., Lewin, E., Grosch, E., ...Schwartz, S. (2014). XMapTools: a MATLAB-based program for electron microprobe X-ray image processing and geothermobarometry. Computers \& Geosciences 62, 227-240.

Leblanc, D., Gleizes, G., Lespinasse, P., Olivier, Ph. \& Bouchez, J.-L. (1994). The Maladeta granite polydiapir, Spanish Pyrenees : a detailed magneto-structural study. Journal of Structural Geology, 16(2), 223-235.

Mansard, N., Raimbourg, H., Augier, R., Précigout, J. \& Le Breton, N. (2018). Large-scale strain localization induced by phase nucleation in mid-crustal granitoids of the south Armorican massif. Tectonophysics, 745, 46-65.

Masci, L., Dubacq, B., Verlaguet, A., Chopin, C., De Andrade, V., \& Herviou, C. (2019). A XANES and EPMA study of $\mathrm{Fe}^{3+}$ in chlorite: importance of oxychlorite and 

implications for cation site distribution and thermobarometry. American Mineralogist. 104(3), 403-417.

Massonne, H-J. \& Schreyer, W. (1987). Phengite geobarometry based on the limiting assemblage with K-feldspar, phlogopite, and quartz. Contributions to Mineralogy and Petrology, 96, 2, 212-224, doi: 10.1007/BF00375235.

McCaig, A. M., Tritlla, J. \& Banks, D.A. (2000). Fluid mixing and recycling during Pyrenean thrusting: evidence from fluid inclusion halogen ratios. Geochimica et Cosmochimica Acta, 64,(19), 3395-3412.

Morad, S., Sirat, M., El-Ghali, M. A., K. \& Mansurbeg, H. (2011). Chloritization in Proterozoic granite from the Äspö Laboratory, southeastern Sweden: record of hydrothermal alterations and implications for nuclear waste storage.Clay Minerals, 46, 495-513.

Oliot, E., Goncalves, P. \& Marquer, D. (2010). Role of plagioclase and reaction softening in metagranite shear zone at mid-crustal conditions (Gotthard Massif, Swiss Central Alps). Journal of Metamorphic Geology, 28, 849-871.

Palazzin, G., Raimbourg, H., Stünitz, H., Heilbronner, R., Neufald, K., \& Précigout, J. Evolution in $\mathrm{H}_{2} \mathrm{O}$ contents during deformation of polycrystalline quartz: An experimental study. Journal of Structural geology, 114, 95-110.

Parneix, J. C., Beaufort, D., Dudoignon, P. \& Meunier, A. (1985). Biotite chloritization process in hydrothermally altered granites. Chemical Geology, 51, 89-101.

Paquette, J.-L., Gleizes, G., Leblanc, D. \& Bouchez, J.-L. (1997). Le granite de Bassiès (pyrénées) : un pluton syntectonique d'âge Westphalien. Géochronologie U-Pb sur zircons. Compte Rendus de l'Académie des Sciences de Paris 324, 387-392.

Rasmussen, B. \& Muhlig, J. R. (2007). Monazite begets monazite: evidence for dissolution of detrital monazite and reprecipitation of syntectonic monazite during low-grade regional metamorphism. Contribution t Mineralogy and Petrology, 154, 675-689. 
873 Román-Berdiel, T., Casas, A. M., Oliva-Urcia, B., Pueyo, E. L. \& Rillo, C. (2004). The main

874

875

876

877

878

879

880

881

882

883

884

885

886

887

888

889

890

891

892

893

894

895

896

Variscan deformation event in the Pyrenees: new data from the structural study of the Bielsa granite. Journal of Structural Geology, 26, 659-677.

Rubatto, D. (2017). Zircon: The Metamorphic Mienral, In: Petrochronology: Methods and Applications, Kohn, M. J., Engi, M. \& Lanari, P. Reviews in Mineralogy \& Geochemistry.

Shea, W. T.J., Kronenberg, A.K. (1993). Strength and anisotropy of foliated rocks with varied mica contents. Journal of Structural Geology, 15, 1097-1121.

Stacey, J. S. \& Kramers, J. D. (1975). Approximation of terrestrial lead isotope evolution by two-stage model. Earth and Planetary Science Letters, 26, 207-221.

Tera, F. \& Wasserburg, G. J. (1972). U-Th-Pb systematics in lunar highland samples from the Luna 20 and Apollo 16 missions. Earth and Planetary Science Letters, 17, 36-51.

Tornos, F., Delgado, A. Casquet, C. \& Galindo, C. (2000). 300 Million years of episodic hydrothermal activity: stable isotope evidence from hydrothermal rocks of the Eastern Iberian Central System. Mineralium Deposita, 35, 551-569.

Tullis, J., Yund, R. \& Farver, J. (1996). Deformation-enhanced fluid distribution in feldspar aggregates and implications for ductile shear zones. Geology, 24(1), 63-66.

Turpin, L., Leroy, J. L. \& Sheppard, S. M. F. (1990). Isotopic systematics (O, H, C, Sr, Nd) of superimposed barren and U-bearing hydrothermal system in a Hercynian granite, Massif Central, France. Chemical Geology, 88, 85-98.

Vacherat, A., Mouthereau, F., Pik, R., Huyghe, D., Paquette, J-L., Christophoul, F., ...Tibari, B. (2017). Rift-to-collision sediment routing in the Pyrenees: A synthesis from sedimentological, geochronological and kinematic constraints. Earth-Sciences Reviews, $172,43-74$. 
897 Vidal, O., De Andrade, V., Lewin, E., Munoz, M., Parra, T., \& Pascarelli, S. (2006). P-T 898 deformation-Fe3+/Fe2+ mapping at the thin section scale and comparison with XANES 899 mapping. Application to a garnet-bearing metapelite from Sambagawa metamorphic belt (Japan). Journal of Metamorphic Geology 24, 669-683.

901

902

903

904

905

906

907

908

909

910

911

912

913

914

915

916 917 this article.

918 Description Supinfo.doc/x

919 Appendix S1. Analytical methods for geochronology and monazite analysis

920 studies of syntectonic veins in the Néouvielle Massif, Pyrenees. Geological Society, London, Special Publications, 144, 129-135.

Wehrens, P., Baumberger, R. Berger, A. \& Herweg, M. (2017). Journal of Structural Geology, $94,47-67$.

Wintsch, R.P., Christoffersen, R. \& Kronenberg, A.K. (1995). Fluid-rock reaction weakening of fault zones. Journal of Geophysical Research, 100(B7), 13021-13032.

Withney, D., \& Evans, B. (2010). Abbreviations for names of rock-forming minerals. American Mineralogist, 95, 185-187.

Yuguchi, T., Sasao, E., Ishibashi, M. \& Nishiyama, T. (2015). Hydrothermal chloritization processes from biotite in the Toki granite, Central Japan: Temporal vairations of the composition of hydrothermal fluids associated with chloritization. American Mineralogist, 100, 1134-1152.

\section{SUPPORTING INFORMATION}

Additional Supporting Information may be found online in the supporting information tab for

Figure S1. Example of location of laser spot for zircon, anatase and titanite dating.

Wayne, D. M. \& McCaig, A. M. (1998). Dating fluid flow in shear zones: Rb-Sr and U-Pb 
921 Figure S2. Tera-Wasserburg diagrams for all analysed anatase, titanite and zircon. Concordia922 discordia diagrams for zircon.

923 Dataset.xls/x

924 Table S1. Rapresentative chemical composition of chlorite and white mica.

925 Table S2. Representative chemical composition of monazite in samples ZA16-98 and ZAL1892611.

927 Table S3. Original istopic data for zircon, titanite and anatase. Graphs represents weighted mean zircon ages.

\section{FIGURE CAPTION}

931 Figure 1 Geological maps and cross section. (a) Schematic structural map of the Pyrenees, 932 modified from Román-Berdiel, Casas, Oliva-Urcia, Pueyo, and Rillo, 2004. Red square: Bielsa, 933 N: Néouvielle, M: Maladeta, J: Jau. (b) Schematic structural cross section of the Axial Zone 934 (modified from Jolivet et al., 2007). Yellow stars indicate limits of the area where pervasive 935 alteration of the original granite has been documented. Black rectangle denotes the studied area. 936 (c) Geological map of the studied area. (d) Cross sections along transects AA' and BB'.

Figure 2 Field structures around Lake Urdiceto. (a) Mylonitic corridor along the transect BB' of Figure 1c. White: schistosity (S), yellow: shear planes (C). (b) Second example of mylonitic 940 corridor a few hundred meters eastward of transect BB', at the contact with the Triassic 941 sediments. Schistosity ( $\mathrm{S}$, filled white lines) is subparallel to S-dipping shear planes (C, marked 942 in yellow) due to high strain. (c) Mylonitic bands within the basement with C planes varying 943 from S-dipping to N-dipping. (d) N-dipping shear planes in a mylonite at the contact between 944 the basement and the sedimentary cover. (e) Enlarged view of mylonitic granite (see panel a for 945 location). (f) Enlarged view of weakly deformed (but altered) granite (see panel a for location). 
947 Figure 3 Field structures in the Triassic sediments. (a) Unconformity between the basement 948 and the Triassic sediments. (b) Chlorite-quartz bearing veins at the contact between the 949 basement and the basal breccia in the Triassic sediments. (c) Two generations of chlorite-quartz 950 bearing veins within Triassic sandstones and pelites.

951

952 Figure 4 Photomicrographs (a, c, e and g) and backscattered electron images (BSE) (panels b, $953 \mathrm{~d}, \mathrm{f}$ and $\mathrm{h}$ ) of granitic basement with increasing deformation. Abbreviations are from Whitney 954 \& Evans (2010), except Wm: white mica; (a) Undeformed but altered granite; (b) BSE image 955 of a Chl $+\mathrm{Wm}+\mathrm{Ttn}$ pseudomorph after Bt; (c) weakly deformed and altered granite with dark 956 Chl-Wm pseudomorphs after Bt folded to form kink bands; (d) BSE images of a kinked Chl 957 flake; (e) weakly deformed sample with opening fractures. Chlorite flakes are cross cut by 958 secondary mm-size fractures. (f) BSE image of a Chl + Ttn flake after Bt (Bt relicts still visible), cross cut by a fracture filled with secondary chlorite; $(\mathrm{g})$ mylonite; (h) folded $\mathrm{Chl}+\mathrm{Wm}+\mathrm{Ant}$ 960 flake cross cut by a secondary fracture in a highly deformed sample. Two types of anatase grains 961 are recognized.

962

963 Figure 5 Backscattered images of accessory minerals in the granitic basement. (a) and (b) 964 magmatic Ilm breaking down to Ant + Cal, then to Ttn. (c) Anatase 1 folded within a Chl flake 965 and replaced by Ttn. (d) Magmatic zircon (core) with metamict rims (low CL intensity) in 966 sample ZA16-119. (e) Chlorite flakes (ChlA) with type-1 Ant cross cut by ChlB-bearing 967 fractures with type-2 Ant (sample ZA16-73). (f) Type-2 Ant rimming titanite after anatase 968 (type-1 Ant, preserved in the core of titanite). (g) Chlorite flake (ChlA) in sample ZA16-79 969 with both types of anatase. (h) Relationships between titanite and anatase in sample ZA16-128. 
970 (i) Monazite in sample ZA16-98. The inset image shows the patchy and porous aspect of the 971 monazite grains. (j) Monazite in reaction halos around fractures in sample ZA16-88.

972

973 Figure 6 Macro- and micro-scale petrography of the Triassic sediments. (a) Hand sample 974 collected at the boundary between sediments and the granitic basement (ZAL18-11). (b) 975 Backscattered electron image of panel a, showing the occurrence of anatase, Fe-oxides and Mnz 976 within neocrystallized $\mathrm{Chl}$ and $\mathrm{Wm}$. The inset show the patchy texture of monazite grains. (c) 977 Chlorite-bearing veins and shear planes forming 'en-echelon' microstructures in sample ZA16-

978 979 980 981 982 983 984 985 986 987 988 989 990 991

992

993 Figure 9 Thermobarometric results. (a) Chlorite temperatures calculated with the method of 994 Vidal et al. (2006) (circles) and Bourdelle et al. (2013) (rectangles). (b) Monte Carlo simulation

Figure 8 Chemical composition of white mica. (a) Compositional triangular plot and trends for white mica in the basement. (b) Compositional ternary diagram of white mica in the sedimentary samples. Mus: muscovite, Cel: celadonite, Pyr: pyrophillite. 12. (d) Two generations of chlorite (in veins and shear planes) in samples ZA16-12 (zoom of panel c). ChlA and ChlB refer to chlorite flakes and chlorite in fractures/veins respectively but may be different in origin to ChlA and ChlB observed in the basement.

Figure 7 Chemical composition of chlorite. (a) Quantified SEM map of $\mathrm{Al}_{2} \mathrm{O}_{3}$ content in chlorite in sample B10-31 (mylonite). (b) Quantified SEM map of FeO content in chlorite of sample ZA16-82 (fractured sample). (c) Elementary Al vs Mg (\%) content of all analysed chlorites; (d) Trends and compositional groups identified in chlorite in samples collected east of Lake Urdiceto; (e) Trends and compositional groups identified in chlorite in samples collected west of Lake Urdiceto. 
995 for the temperature dataset calculated for basement chlorite (3000 iterations, see the text for 996 details). (c) Pressure range estimated from the celadonite content in white mica, following the 997 calibration of Massonne and Schreyer (1988). This calibration does not consider the pyrophillite 998 content in white mica, as it is the case in the Bielsa granite. Pressure values should therefore be 999 considered as minimum values.

1000

1001 Figure 10 Geochronology results. (a) Representative Tera-Wasserburg concordia lower 1002 intercept age defined by titanite analyses in an undeformed sample (ZA16-84). (b) Tera1003 Wasserburg Concordia lower intercept ages defined by various anatase populations (red: old, 1004 blue: young) in a deformed sample (ZA16-128). (c) Frequency distribution of monazite ages.

1005 Note that youngest monazite ages are estimated from $\mathrm{Pb}$ depleted domains $(\mathrm{Pb}<\mathrm{EPMA}$ 1006 detection limits of $\sim 100 \mathrm{ppm}$ ) and monazite-thorite domains (see the text for details). Note the 1007 ages calculated for domains where $\mathrm{Pb}$ is below the EPMA detection limit are maximum ages. 1008 (d) Summary of geochronological results (this study) integrated with previously published data 1009 for metamorphic events in the Axial zone. U-Pb Ant-Ttn indicate the ages obtained for analysis 1010 where a titanite locally preserved anatase cores (type-1 Ant, see the text for details). (e) U and 1011 Th content of monazite in sample ZAL18-11. (f) Probability density plot of all zircon ages for

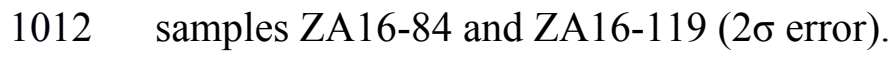

1013

1014 Table 1 Summary of sample descriptions and ages. 